\title{
Results on twisted sums of Banach and operator spaces
}

\author{
Willian Hans Goes Corrêa
}

TESE APRESENTADA

$\mathrm{AO}$

Instituto DE MATEMÁticA E EstatísticA

DA

UniversidADE DE SÃo PAUlo

PARA

OBTENÇÃO DO TÍTULO

$\mathrm{DE}$

Doutor EM CiÊnCIAS

Programa: Matemática

Orientador: Prof. Dr. Valentin Raphael Henri Ferenczi

Durante o desenvolvimento deste trabalho o autor recebeu auxílio financeiro da CAPES e do $\mathrm{CNPq}$

São Paulo, abril de 2018 



\section{Results on twisted sums of Banach and operator spaces}

Esta versão da tese contém as correções e alterações sugeridas pela Comissão Julgadora durante a defesa da versão original do trabalho, realizada em 26/02/2018. Uma cópia da versão original está disponível no

Instituto de Matemática e Estatística da Universidade de São Paulo.

Comissão Julgadora:

- Prof. Dr. Valentin Raphael Henri Ferenczi - IME-USP

- Prof ${ }^{\mathrm{a}}$. Dra ${ }^{\mathrm{a}}$. Christina Brech - IME-USP

- Prof. Dr. Daniel Marinho Pellegrino - UFPb

- Prof. Dr. Jesús Manuel Trinidad Fernandez Castillo - UEx

- Dr. Wilson Albeiro Cuellar Carrera 

To Him who loves us 

"A woman when she is in travail hath sorrow, because her hour is come: but as soon as she is delivered of the child, she remembereth no more the anguish, for joy that a man is born into the world." 

Many people have been helping me in the process of delivering this child. Now it is time to thank them.

Firstly I must thank those who gave me the financial support I needed during gestation. Thank you, CAPES and CNPq.

I also would like to thank Antonio de Padua Franco Filho for presenting me to my advisor and for many pleasant conversations.

I am most indebted to Valentin Ferenczi, my advisor, who so many times made the process of researching more than joyful. I thank him for his help and his smile. Also, he presented me to a wonderful video of a cat and an owl playing together.

I thank my co-advisor, Christina Brech, for always being available when I needed help, be it mathematically or professionally. She and Ivan Struchiner read an embryonic form of this work when they were part of my qualification committee, and gave me valuable suggestions and insights. I could also turn to Ivan when I needed personal help. Thank you both a lot.

My family was always present when I needed them, and received me with open arms whenever I needed a break from the big city. Thank you a lot for your love.

My closest friends helped me to keep my mental stability more times than they can imagine (for better or for worse). Having you with me makes all the difference in the world. Thank you, my broas.

I met amazing people at the ABU of IME. They helped me to spiritually survive when I could have been alone. Of these, I must especially thank Felipe Felix, Ananda Gama, Beatriz Ibrahim and Shayenne Moura, for they "stayed with me in my trials".

During the writing of this thesis I spent almost an year in Badajoz, at Universidad de Extremadura. I thank the university administration for making an office and a computer available for me during this time. The professors there gave me a great welcome and paid me many coffees. I am very grateful for their sympathy.

Their group of functional analysis also provided me with a computer I could use to write this thesis at home. Thank you very much, for this and for many enjoyable conversations and lunches.

I especially thank Jesús Castillo. He read a preliminary version of this thesis and gave many useful comments. He also provided me with books and movie suggestions, and a first edition of Alfred Bester! His warmness is one of the fondest memories I will take from Badajoz.

During my time there I also had the opportunity to spend more time with one of my scientific brothers, Wilson Cuellar. Knowing him better is one of the gifts this period gave me. He, João Fernando da Cunha, Gilles Godefroy, Gilles Pisier and Yanqi Qiu also helped me mathematically, and I am grateful to them.

The committee board of my thesis defense will help me to clean the baby, so I thank them in advance.

Above all, I thank God for giving me all these wonderful people.

Now, if you excuse me, I can already feel the contractions... 



\section{Resumo}

Corrêa, W. H. G. Resultados de somas torcidas de espaços de Banach e espaços de operadores. 2018. 157 f. Tese (Doutorado) - Instituto de Matemática e Estatística, Universidade de São Paulo, São Paulo, 2018.

Neste trabalho estudamos somas torcidas induzidas por interpolação complexa, tanto de espaços de Banach como de espaços de operadores. Na primeira parte da tese focamos em espaços de Banach, e esclarecemos como a interpolação de famílias, assim como a de pares, gera uma extensão do espaço interpolado, chamada de espaço derivado. Estudamos como os tipos e cotipos dos espaços sendo interpolados influenciam na trivialidade ou singularidade do espaço derivado, e aplicamos os resultados para o estudo de submódulos das classes de Schatten e para a obtenção de somas torcidas não-triviais em que os três espaços da sequência exata curta não possuem a propriedade da aproximação. Na segunda parte, desenvolvemos a teoria de somas torcidas na categoria de espaços de operadores, e apresentamos vários exemplos de somas torcidas completamente singulares e completamente não-triviais nessa categoria. Em particular, resolvemos duas versões do problema de Palais para espaços de operadores.

Palavras-chave: somas torcidas, interpolação complexa, espaços de operadores. 



\section{Abstract}

Corrêa, W. H. G. Results on twisted sums of Banach and operator spaces. 2018. $157 \mathrm{f}$. Thesis (PhD) - Instituto de Matemática e Estatística, Universidade de São Paulo, São Paulo, 2018.

In this work we study twisted sums induced by complex interpolation, of Banach spaces as well as of operator spaces. In the first part of the thesis we focus on Banach spaces, and clarify how interpolation of families, as of couples, induces an extension of the interpolation space, called the derived space. We study how the types and cotypes of the spaces being interpolated determine the triviality or singularity of the derived space, and we apply the results to the study of submodules of the Schatten classes and in the obtainment of nontrivial twisted sums in which all of the three spaces in the short exact sequence do not have the approximation property. In the second part we develop the theory of twisted sums in the category of operator spaces and present many examples of twisted sums which are completely singular and completely nontrivial. In particular, we solve two versions of Palais' problem for operator spaces.

Keywords: twisted sums, complex interpolation, operator spaces. 



\title{
LIST OF ABBREVIATIONS AND ACRONYMS
}

\author{
3SP ..... 3-space property \\ c. s. c. .... completely strictly cosingular \\ c. s. s. .... completely strictly singular \\ C3SP .... complete 3-space property \\ o. s. s. .... operator space structure \\ UMD .... uniform martingale property
}



$\tilde{q}-\tilde{q}(x+\operatorname{ker}(q))=q(x)$

$\Sigma(\bar{X})$ - sum space of a compatible couple $\bar{X}$

$\Delta(\bar{X})$ - intersection space of a compatible couple $\bar{X}$

$\mathbb{S}-\{z \in \mathbb{C}: 0<\operatorname{Re}(z)<1\}$

$\mathbb{S}_{j}-\{z \in \mathbb{C}: \operatorname{Re}(z)=j\}, j=0,1$

$\mathbb{D}$ - open unit disk

$\mathbb{T}$ - unit circle

$d \lambda$ - Lebesgue measure on $\mathbb{T}$

$d z-\frac{1}{2 \pi} d \lambda$

$P_{z_{0}}$ - Poisson kernel with respect to $z_{0}$

$\tilde{P}_{z_{0}}$ - conjugate Poisson kernel with respect to $z_{0}$

$\log ^{+} x-\max \{0, \log x\}$

$N$ - Nevanlinna class

$H^{p}$ - Hardy spaces, $1 \leq p \leq \infty$

$N^{+}$- Smirnov class

$\mu_{z_{0}}$ - harmonic measure induced on the boundary of a given domain by $z_{0}$

$\mu_{z_{0}}^{j}$ — probability measure induced on $\mathbb{S}_{j}$ by $\mu_{z_{0}}, j=0,1$

$T_{p}^{X}$ — best type $p$ constant of $X$

$p_{x}$ - supremum of the types of $X$

$C_{q}^{X}$ - best cotype $q$ constant of $X$

$q_{X}$ - infimum of the cotypes of $X$

$\left(r_{k}\right)$ - Rademacher functions

$B_{X}$ - closed unit ball of $X$

$A \otimes x-\left(a_{i, j} x\right)$, where $A=\left(a_{i, j}\right)$

$R$ - row operator space

$C$ - column operator space

$C B(X, Y)$ - space of completely bounded operators from $X$ into $Y$

$\mathrm{OH}$ - operator Hilbert space

$\odot$ - matrix inner product

$\otimes^{h}$ - Haagerup tensor product

$\hat{\otimes}$ - projective tensor product of operator spaces

$\mathbb{M}_{\infty}(X)$ - linear space of infinite matrices with entries in $X$

$\pi_{X}^{n}$ - truncation map from $\mathbb{M}_{\infty}(X)$ into $M_{n}(X)$

$M_{\infty}(X)-\left\{x \in \mathbb{M}_{\infty}(X)\right.$ such that $\left.\sup _{n}\left\|\pi_{X}^{n}(x)\right\|<\infty\right\}$

$\mathscr{K}(X)$ - closure of $\cup_{n} M_{n}(X)$ in $M_{\infty}(X)$ 
$\mathscr{K} \otimes_{\min } X-\mathscr{K}(X)$

$S_{p}^{n}-p$-Schatten class of dimension $n$

$C S S(X, Y)$ - set of completely strictly singular operators from $X$ into $Y$

$\operatorname{CSC}(X, Y)$ - set of completely strictly cosingular operators from $X$ into $Y$

$R(\theta)-(R, C)_{\theta}$

$o(X)(\theta)-(\min (X), \max (X))_{\theta}$

$o(X)-o(X)\left(\frac{1}{2}\right)$

$d O H-d R\left(\frac{1}{2}\right)$ 
INTRODUCTION ....................... 1

TWISTED SUMS OF BANACH SPACES . . . . . . . . . . . 9

3-Space Problems ................... 9

Twisted sums and quasilinear maps ............... 11

Complex Interpolation . . . . . . . . . . . . . . . . . . 15

2.3.1 Complex Interpolation of Couples . . . . . . . . . . . . . . . 15

2.3.2 Complex Interpolation of Families . . . . . . . . . . . . . . . . 20

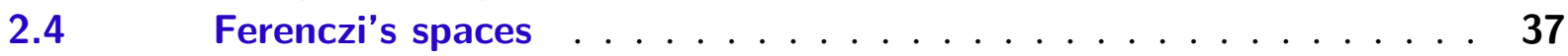

2.5 Complex interpolation of couples through $L_{p}$-norms $\ldots \ldots$

3 TYPE, COTYPE AND TWISTED SUMS INDUCED BY COMPLEX INTERPOLATION . . . . . . . . . . . . . . . . . . . 41

$3.1 \quad$ Type and cotype of a Banach space . . . . . . . . . . 41

3.2 Type and twisted sums induced by interpolation . . . . . . . . 46

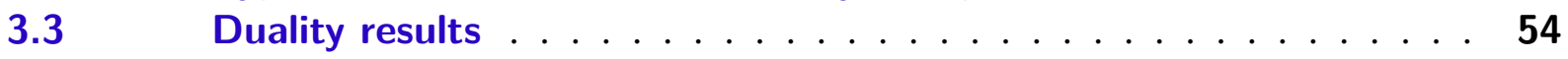

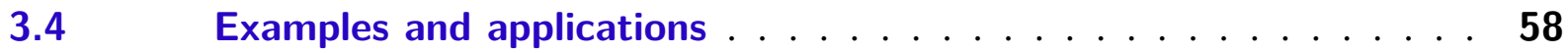

$3.4 .1 \quad \ell_{p}$ spaces $\ldots \ldots \ldots \ldots \ldots \ldots \ldots$

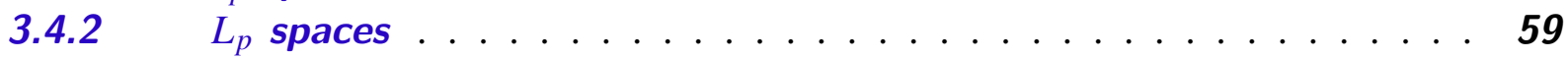

3.4.3 Ferenczi's spaces revisited . . . . . . . . . . . . . 59

3.4.4 Schatten classes . . . . . . . . . . . . . . . . . 60

3.4.5 Spaces without the approximation property . . . . . . . . . 61

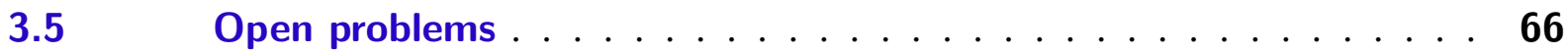

II TWISTED SUMS OF OPERATOR SPACES $\quad 67$

$4 \quad$ OPERATOR SPACES . . . . . . . . . . . . . . . . . . . . 69

$4.1 \quad$ First definitions and examples . . . . . . . . . . . . . 69

4.2 Operator space analogues of Banach space constructions . . . . 71

4.2.1 Complex interpolation . . . . . . . . . . . . . . . 73

$4.3 \quad \min$ and $\max$ operator spaces . . . . . . . . . . . 74

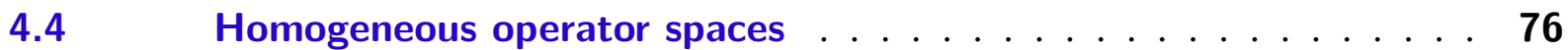

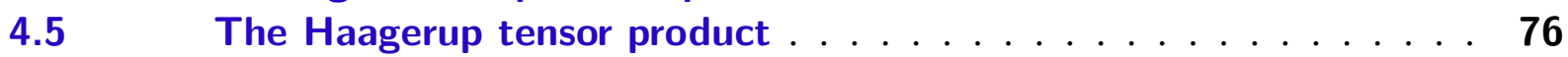

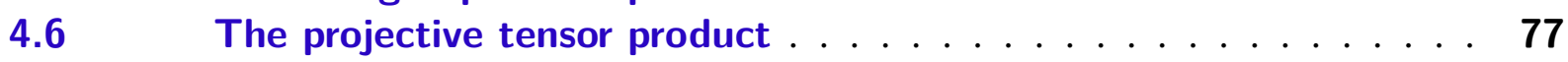

$4.7 \quad L_{p}$ operator spaces $\ldots \ldots \ldots \ldots 77$

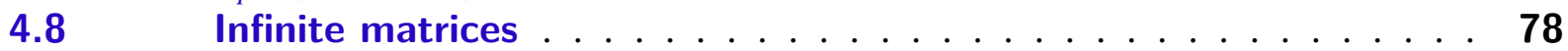

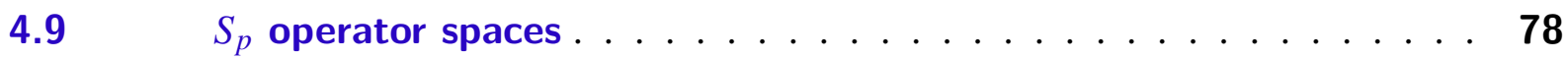

$4.10 \quad$ Operator algebras ..................... 79 


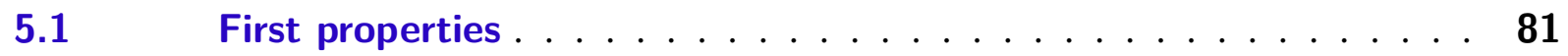

$5.2 \quad$ Twisted sums of the matrices spaces . . . . . . . . . 85

5.3 Completely stricly singular and completely strictly cosingular maps 86

$5.4 \quad$ Categorical constructions . . . . . . . . . . . . . 91

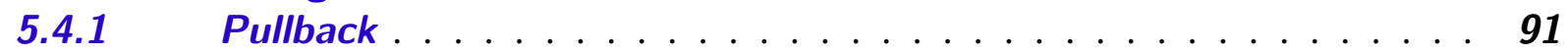

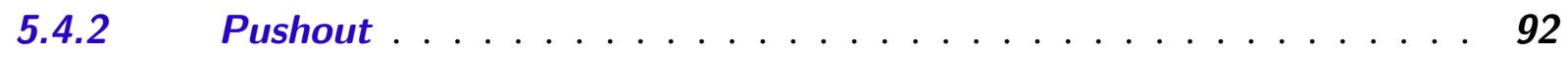

$5.5 \quad$ Complete twisted sums induced by complex interpolation . . . . . 95

5.6 Complex interpolation of operator spaces through $L_{p}$-norms $\ldots 100$

$5.6 .1 \quad$ Duality ... . . . . . . . . . . . . . . . . . 103

5.7 Complete Exact Sequences and the Haagerup Tensor Product . . 106

6 CONCRETE TWISTED SUMS OF OPERATOR SPACES . . . . . 111

6.1 The operator space structure of the Kalton-Peck spaces . . . . . . 111

$6.2 \quad$ Twisting $\min$ and $\max \ldots \ldots \ldots \ldots \ldots 12 \ldots \ldots \ldots \ldots$

$6.3 \quad$ Noncommutative $L_{p}$ spaces . . . . . . . . . . . . . . . 112

6.4 The spaces $R(\theta)$ and Palais' problem for operator spaces . . . . 113

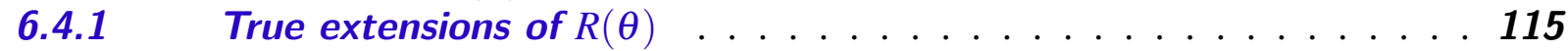

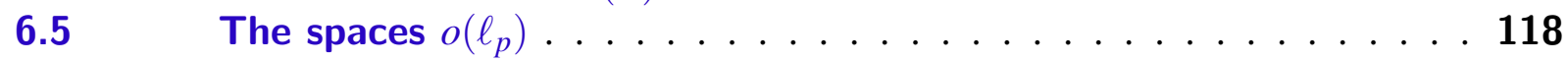

6.6 Complete extensions of $O H \ldots \ldots \ldots \ldots \ldots$

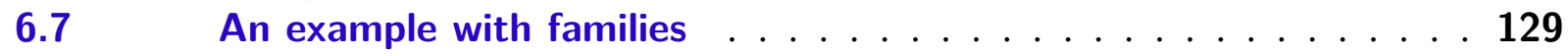

$6.8 \quad$ Saturation . . . . . . . . . . . . . . . 131

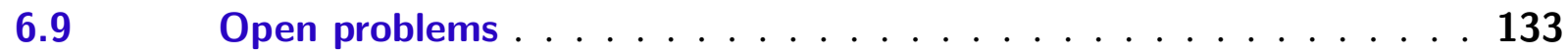

BIBLIOGRAPHY . . . . . . . . . . . . . . . . . . 135 
How is a Banach space determined by its subspaces and quotients? Or, in other words, which properties of a subspace and of its respective quotient determine the properties of a Banach space? For example, suppose that $X$ is a Banach space with a closed subspace $Y$, and suppose that $Y$ and $X / Y$ have any of the following properties:

- Nontrivial type;

- Nontrivial cotype;

- Reflexivity;

- Is isomorphic to $\ell_{1}$;

- Is isomorphic $\ell_{\infty}$.

Then $X$ also has the same property. These properties are said to be 3-space properties (3SP, for short. See (CASTILLO; GONZÁLEZ, 1997) for proofs of these results). On the other hand, for any of the following properties there is a Banach space $X$ with a subspace $Y$ such that $Y$ and $X / Y$ have the property, but $X$ has not:

- Is isomorphic to $\ell_{p}$, for some $p \in(1, \infty)$ (same $p$ for $Y$ and $X / Y$ );

- Is a UMD space;

- Has type 2;

- Has cotype 2;

- Has the approximation property.

In other words, $X$ is not properly determined by its subspaces and quotients when one considers any of these properties (again, we refer to (CASTILLO; GONZÁLEZ, 1997)).

To prove that any of the properties of the second group are not 3SP, one must find an example of $X$ and $Y$, which may turn out to be quite a herculean task (see for example the proof that being a UMD space is not a 3SP from (KALTON, 1992)). Notice that if the property being considered is invariant by isomorphism finding such example is the same as finding a short exact sequence of Banach spaces and bounded linear maps

$$
0 \longrightarrow Y \stackrel{i}{\longrightarrow} X \stackrel{q}{\longrightarrow} Z \longrightarrow 0
$$

where $Y$ and $Z$ have the property, and $X$ has not. Such an $X$ is said to be a twisted sum of $Y$ and Z.

Given two Banach spaces $Y$ and $Z$, there is an obvious and trivial twisted sum of $Y$ and $Z$ : the Banach space $Y \oplus Z$. Of course, this in not really interesting, since in most cases the direct sum will not be an example of the failure of the 3SP. So one is led to the question: how to obtain nontrivial twisted sums of Banach spaces? 
We will consider the following approach of Kalton and Peck (KALTON; PECK, 1979): given two Banach spaces $Y$ and $Z$, linearly the space $X$ will be $Y \times Z$, and we must 'twist' the norm by some map $F: Z \rightarrow Y$ :

$$
\|(y, z)\|_{F}=\|y-F z\|_{Y}+\|z\|_{Z}
$$

for every $(y, z) \in Y \times Z$. (We note that there is another approach from (SMIRNOV; KHUEN, 1991; SMIRNOV; SHEIKHMAN, 1991): the norm is always the direct sum norm, but the operations are twisted). As pointed out by Jesús Castillo ${ }^{1}$, the questions now are:

- Which maps $F$ may be used?

- How to obtain such maps?

- When is $Y$ complemented in $X$, i. e., when is $X$ a trivial twisted sum of $Y$ and $Z$ ?

Regarding the first question, let us consider a consequence of the triangle inequality. If $\|\cdot\|_{F}$ is a norm, we should have

$$
\begin{aligned}
\left\|F\left(z_{1}+z_{2}\right)-F\left(z_{1}\right)-F\left(z_{2}\right)\right\|_{Y} & \leq\left\|\left(F\left(z_{1}\right), z_{1}\right)+\left(F\left(z_{2}\right), z_{2}\right)\right\|_{F} \\
& \leq\left\|\left(F\left(z_{1}\right), z_{1}\right)\right\|_{F}+\left\|\left(F\left(z_{2}\right), z_{2}\right)\right\|_{F} \\
& =\left\|z_{1}\right\|_{Z}+\left\|z_{2}\right\|_{Z}
\end{aligned}
$$

that is, $F$ should satisfy a quasilinearity condition

$$
\left\|F\left(z_{1}+z_{2}\right)-F\left(z_{1}\right)-F\left(z_{2}\right)\right\|_{Y} \leq\left\|z_{1}\right\|_{Z}+\left\|z_{2}\right\|_{Z}
$$

for every $z_{1}, z_{2} \in Z$. When one starts to look for maps satisfying this condition, it is found that this is quite restrictive, and the 'right' condition should be

$$
\left\|F\left(z_{1}+z_{2}\right)-F\left(z_{1}\right)-F\left(z_{2}\right)\right\|_{Y} \leq C\left(\left\|z_{1}\right\|_{Z}+\left\|z_{2}\right\|_{Z}\right)
$$

for some constant $C>0$ and every $z_{1}, z_{2} \in Z$. In doing so, we lose the triangle inequality, which is substituted by

$$
\left\|\left(y_{1}, z_{1}\right)+\left(y_{2}, z_{2}\right)\right\|_{F} \leq K\left(\left\|\left(y_{1}, z_{1}\right)\right\|_{F}+\left\|\left(y_{2}, z_{2}\right)\right\|_{F}\right)
$$

for some constant $K>0$ and every $\left(y_{1}, z_{1}\right),\left(y_{2}, z_{2}\right) \in Y \times Z$. So, in studying twisted sums of Banach spaces we are led out of the locally convex world! Instead of considering norms, we must consider quasinorms, where the triangle inequality is replaced by a scaled triangle equality. Fortunately, most of the times (for example, if $Z$ has type bigger than 1) the quasinorm on the twisted sum is equivalent to a norm.

The answer to the first question is that the maps we must work with are homogeneous maps satisfying (1.1), called quasilinear maps.

Once we know which maps we should look for, there is the problem of actually finding quasilinear maps. It is an evidence of the genius and hard work of the pioneers of the theory, Enflo, Lindenstrauss, Pisier (ENFLO; LINDENSTRAUSS; PISIER, 1975), Kalton (KALTON, 1978) and Peck (KALTON; PECK, 1979), and Ribe (RIBE, 1979), that they found quasilinear maps without the aid of complex interpolation.

In the present work we will only consider twisted sums induced by complex interpolation, which generates quasilinear maps. For those already acquainted with complex interpolation, if we have an interpolation couple $\left(X_{0}, X_{1}\right)$, then for $0<\theta<1$ we have a quasilinear map

1 In his presentation Stability properties of the differential process generated by complex interpolation at the Universitat Politècnica de Valencia, Spain, Conference on Nonlinear Functional Analysis, 2017. 
$\omega_{\theta}=\Omega_{\theta}-L_{\theta}: X_{\theta} \rightarrow X_{\theta}$ where $\Omega_{\theta}$ (the factor that really matters) is found in the following way: for each $x \in X_{\theta}$ take $B(x) \in \mathscr{F}$ (the Calderón space of the couple $\left(X_{0}, X_{1}\right)$ ) such that $\|B(x)\| \leq 2\|x\|_{\theta}$ homogeneously. Then $\Omega_{\theta}(x)=(B(x))^{\prime}(\theta)$.

For example, considering the interpolation couple $\left(\ell_{\infty}, \ell_{1}\right)$ one has $\left(\ell_{\infty}, \ell_{1}\right)_{\frac{1}{2}}=\ell_{2}$ and for $x \in c_{00}$

$$
\Omega_{\frac{1}{2}}(x)=2 \sum x_{n} \log \frac{\left|x_{n}\right|}{\|x\|_{2}} e_{n}
$$

which is a multiple of the quasilinear map found in (KALTON; PECK, 1979) without interpolation considerations. Kalton and Peck had the work of proving the hard way that this map is quasilinear, while this is automatic when one sees it as induced by complex interpolation.

So, for us, the answer to the second question will be 'through complex interpolation'. Actually, due to results of (KALTON, 1992), in many situations this is essentially the only way to obtain a quasilinear map, that is to say, that given a space $X$ and a quasilinear map $\Omega: X \rightarrow X$ satisfying certain conditions, then $\Omega$ must be equivalent in an appropriate sense to a quasilinear map induced by interpolation.

Finally, the last question: once we obtain a quasilinear map and build the space $X$, how do we know if after all this work we did not actually end up with the trivial twisted sum?

We begin by noticing that if our quasilinear map $F$ used for building $X$ is linear then it is easy to check that $Y$ is complemented in $X$. So linearity is not good for our purposes. Is a nonlinear quasilinear map enough to guarantee that $Y$ is not complemented in $X$ ? It is not, since it is also possible to prove that a bounded quasilinear map will induce the trivial twisted sum.

It turns out that to obtain a nontrivial twisted sum we must find a quasilinear map that is far from a linear one, in the sense that there is no linear map $L: Z \rightarrow Y$ and $C>0$ such that

$$
\|F(z)-L(z)\|_{Y} \leq C\|z\|_{Z}
$$

for every $z \in Z$. Such an $F$ is called nontrivial.

The first part of this work is devoted to finding criteria for the nontriviality of the quasilinear map induced by complex interpolation.

As we delve deeper into the theory of twisted sums it becomes apparent that a special role is played by quasilinear maps that are extremely far from linear ones, i. e., quasilinear maps $F: Z \rightarrow Y$ such that $\left.F\right|_{W}: W \rightarrow Y$ is never trivial for any closed infinite dimensional subspace $W$ of $Z$. Such quasilinear maps are called singular. The Kalton-Peck map (1.2) is an example of singular quasilinear map.

The importance of singular quasilinear maps is found, for example, in the following result: a hereditarily indecomposable Banach space $X$ is a space such that we may never write $X=Y \oplus Z$ for any closed infinite dimensional subspaces $Y, Z \subset X$. A twisted sum of hereditarily indecomposable Banach spaces is itself hereditarily indecomposable if and only if any quasilinear map that defines it is singular (CASTILLO; FERENCZI; GONZÁLEZ, 2017). Also, recent work (CASTILLO et al., 2017b) indicates that the singularity of the Kalton-Peck map is related to the question of whether the Kalton-Peck space is isomorphic to its hyperplanes. In this work we also study the singularity of quasilinear maps induced by interpolation.

This thesis is divided in two parts. The first one focuses on twisted sums of Banach spaces. In the second part we change the category of Banach spaces to that of operator spaces.

Operator spaces, or quantum Banach spaces, are the 'noncommutative version of Banach spaces'.

To get the grasp of it, we must think about the foundations of quantum mechanics. In classical mechanics the quantities of interest are measured through functions: a particle has 
a well-defined position, or potential energy, kinetic energy, or whatever is of interest for the problem at hand, as a function with respect to time.

In quantum mechanics the quantities of interest are vectors in a Hilbert space, and accordingly evolve in time according to operators on this Hilbert space. So we pass from functions to operators.

Recall that every Banach space may be seen as a function space on a compact set: if $x \in X$ then it may be seen as a continuous function on the unit ball $B_{X^{*}}$ of $X^{*}$ (endowed with the $w^{*}$-topology) by the formula:

$$
x\left(x^{*}\right)=x^{*}(x)
$$

for every $x^{*} \in B_{X^{*}}$. By the Hahn-Banach Theorem this defines a linear isometry from $X$ into $C\left(B_{X^{*}}\right)$. So a Banach space is by nature a commutative object. Operator space theory is a shift of view regarding Banach spaces; instead of seeing them as spaces of functions on a compact set we see them as spaces of operators on a Hilbert space, i. e., we consider linear isometric embeddings from $X$ into $B(H)$, where $H$ is a Hilbert space. In practice, this means that now we consider a norm on the tensor product $\mathscr{K} \otimes X$, where $\mathscr{K}$ is the space of compact operators on $\ell_{2}$, or either a sequence of norms $\left(\|\cdot\|_{n}\right)$ on the spaces $M_{n}(X)$, and the notion of isomorphism is substituted by that of complete isomorphism.

Nowadays operator space theory is a well established theory, with applications from harmonic analysis to quantum information theory. Being inspired on Banach space theory, much of the latter passes to the former: duality theory, complex interpolation and tensor product theory are just a few examples. Our aim in the second part of this work is to start a systematic study of twisted sums in the category of operator spaces.

We call attention to the fact that short exact sequences of operator spaces are not a new topic in the literature. As usual, they are present, implicitly or explicitly, whenever one talks about subspaces or quotient spaces. They also appear in (PAULSEN, 1998), (WOOD, 1999 (PhD thesis)) and (FORREST; WOOD, 2001), under the hypothesis of splitting in the operator space category. So our work differs from those in two senses: first, we wish to develop the theory of twisted sums of operator spaces inspired by that of Banach spaces, and we are primarily interested in twisted sums which do not split.

To reach our goal we tackle the operator space version of the problem that started the theory of twisted sums of Banach spaces, Palais' problem.

Palais' problem asks whether being isomorphic to a Hilbert space is a 3SP, and was proved to be false by Enflo, Lindenstrauss and Pisier (ENFLO; LINDENSTRAUSS; PISIER, 1975). There is an operator space, the operator Hilbert space, which plays the role of $\ell_{2}$ in operator space theory. We show that being completely isomorphic to the operator Hilbert space is not a 3SP in the category of operator spaces $X$ such that $X$ as a Banach space is a Hilbert space.

More generally, following a suggestion of Gilles Pisier, we call a true twisting of an operator space $X$ an operator space $Z$ such that

- $Z$ has a subspace $Y$ completely isomorphic to $X$;

- $Z / Y$ is completely isomorphic to $X$;

- $Y$ is complemented in $Z$ but is not completely complemented.

We find such space through interpolation theory. As we said, complex interpolation is defined for operator spaces. If we consider finding the interpolation space as the 0-level of interpolation theory, then in this work we develop the 1-level, studying the derivation process in scales of complex interpolation of operator spaces.

We now describe the organization of the thesis: after this introduction, we start the first 
part of the thesis: 'Twisted sums of Banach spaces'.

In Chapter 2 we give the necessary background on twisted sums. We give some examples of $3 \mathrm{SP}$ for illustrative purposes, and state without proof the main results on quasilinear maps and twisted sums, and how complex interpolation of couples of Banach spaces induce twisted sums. Then, we present the complex method of interpolation of families of Banach spaces due to (COIFMAN et al., 1982). We actually give a slight different definition from (COIFMAN et $a l ., 1982)$, because we allow one to choose a dense subspace playing the role of the intersection space, since calculating the actual intersection of the family might be really tricky, and in many cases there is a natural subspace that plays the role of intersection. We illustrate this last point showing how Ferenczi's spaces (FERENCZI, 1997) may be obtained from this framework. Then we discuss how the method for families also induces a twisted sum of the interpolated space. We clarify a point from (ROCHBERG, 1996) and from (KALTON; MONTGOMERY-SMITH, 2003), describing the families for which the pushout method may indeed be used to induce a twisted sum.

In Chapter 3 we give criteria for triviality and singularity of the twisted sum induced by complex interpolation using the classical concepts of Rademacher type and cotype. We recall these concepts and discuss their relationship to complex interpolation through classical results. We also discuss how the results from (CASTILLO; FERENCZI; GONZÁLEZ, 2017) suggest the use of type and cotype to the study of twisted sums. We show that the criteria we obtain are optimal, and apply them to the study of submodules of Schatten classes. We also obtain nontrivial twisted sums of Banach spaces in which none of the spaces in the short exact sequence have the compact approximation property.

Now, the second part, on twisted sums of operator spaces, starts. Chapter 4 presents the background on the theory of operator spaces (we refer to (EFFROS; RUAN, 2000) and (PISIER, 2003) for details). In Chapter 5 we give the first definitions and results regarding twisted sums in the operator space category. We avoid the use of quasilinear maps by showing that in this context complex interpolation also induces twisted sums of operator spaces. We prove a duality result for twisted sums induced by complex interpolation and obtain results regarding the Haagerup tensor product. We also define completely strictly singular and complete strictly cosingular operators and prove properties of these operators analogous to the Banach space case.

Chapter 6 is devoted to applying the techniques from Chapter 5 to obtain twisted sums of operator spaces. In particular, we solve Palais' problem for operator spaces, showing the existence of a Hilbertian operator space which is not completely isomorphic to $\mathrm{OH}$ and has a subspace completely isometric to $O H$ and with quotient also completely isometric to $O H$. We also give examples of families of other twisted sums of operator spaces which are trivial as twisted sums of Banach space but do not split in the operator space category. We also discuss the fact that we actually obtain two different solutions to Palais' problem for operator spaces, and show that the corresponding operator space property for saturation is not a 3SP property in this category.

The main original results of the thesis were first presented in (CORREAA, 2018a; CORRÊA, 2018b).

The author would like to thank both CNPq and CAPES for the financial support he received during his $\mathrm{PhD}$. research. During the development of this work the author received from CNPq (Coordenação de Aperfeiçoamento de Pessoal de Nível Superior - Brazil) the grant 140413/2016-2, and from CAPES (Conselho Nacional de Desenvolvimento Científico e Tecnológico - Brazil) the grants 1328372 and 88881.134107/2016-0, the last one being part of the PDSE program (Programa de Doutorado Sanduíche no Exterior). 



\section{Part I}

Twisted sums of Banach spaces 



\section{TWISTED SUMS OF BANACH SPACES}

In this chapter we present the necessary background on twisted sums and complex interpolation. In the first section we discuss the motivation for the study of twisted sums of Banach spaces through 3-space problems, and in the next section we describe twisted sums through quasilinear maps. A reference for these subjects is (CASTILLO; GONZÁLEZ, 1997).

In Section 2.3 we present complex interpolation of Banach spaces and show how it naturally induces twisted sums. We first present the classical method of interpolation of couples, due to Calderón and Lions (LIONS, 1960; CALDERÓN, 1964), and then the method of interpolation for families of Banach spaces of Coifman et al. (COIFMAN et al., 1982). We give the proofs for most of the results on the method for families for a number of reasons: first, it is not as known as the method for couples; also, we modified slightly the original method from (COIFMAN et $a l ., 1982$ ); finally, the existence of twisted sums induced by interpolation of families is not as straightforward as for interpolation of couples, so we clarify comments regarding this existence from (ROCHBERG, 1996) and (KALTON; MONTGOMERY-SMITH, 2003).

Since we shall work with analytic functions, in this thesis we only consider complex Banach spaces.

\subsection{3-Space Problems}

Many properties of Banach spaces pass to their subspaces and quotients. For example, reflexivity, separability, having finite dimension or being a Hilbert space are all properties inherited by subspaces and quotients. It is then natural to ask for a kind of a converse, i. e., to what extent subspaces and quotients determine the properties of a Banach space.

For example, it is well known that reflexivity of a subspace and the respective quotient implies the reflexivity of the whole space.

Properties determined in this way by subspaces and quotients are called 3-Space Properties.

Definition 2.1.1 (3-Space Properties). A property $P$ of Banach spaces is said to be a 3-space property (3SP, for short) if whenever a subspace $Y$ of a Banach space $X$ and the corresponding quotient $X / Y$ have $P$ then it follows that $X$ has $P$.

A 3-space problem consists in deciding if a given property is or is not a 3-space property.

A natural concept that arises in the study of 3-space properties is that of short exact sequence. 
Definition 2.1.2 (Short Exact Sequence). A short exact sequence of Banach spaces is a diagram

$$
0 \longrightarrow Y \stackrel{i}{\longrightarrow} X \stackrel{q}{\longrightarrow} Z \longrightarrow 0
$$

where $Y, X$ and $Z$ are Banach spaces, the arrows are operators and the kernel of each arrow coincides with the image of the previous one, $i$. e., $i$ is injective, $q$ is surjective, and $\operatorname{ker}(q)=\operatorname{Im}(i)$.

Notice that if $Y$ is a subspace of $X, j$ is the inclusion of $Y$ in $X$, and $\pi: X \rightarrow X / Y$ is the quotient map, we have a short exact sequence

$$
0 \longrightarrow Y \stackrel{j}{\longrightarrow} X \stackrel{\pi}{\longrightarrow} X / Y \longrightarrow 0
$$

On the other hand, given a short exact sequence

$$
0 \longrightarrow Y \stackrel{i}{\longrightarrow} X \stackrel{q}{\longrightarrow} Z \longrightarrow 0
$$

we have from $\operatorname{ker}(q)=\operatorname{Im}(i)$ that $i(Y)$ is closed and, since $i$ is injective, by the Open Mapping Theorem, it is an isomorphism onto its image, i. e., $Y$ is isomorphic to a subspace of $X$. Besides, $q$ induces an operator $\tilde{q}: X / i(Y) \rightarrow Z$ by $\tilde{q}(x+i(Y))=q(x)$, which, again by the Open Mapping Theorem, is an isomorphism between $X / i(Y)$ and $Z$.

The previous discussion implies the following result.

Proposition 2.1.3. Let $P$ be a property of Banach spaces which is stable under isomorphisms. Then $P$ is a 3SP if and only if for every short exact sequence of Banach spaces

$$
0 \longrightarrow Y \longrightarrow X \longrightarrow Z \longrightarrow 0
$$

in which $Y$ and $Z$ have $P$, the space $X$ has $P$ as well.

Notation 2.1.4. If $q: X \rightarrow Z$ is a surjective linear map then $\tilde{q}: X / \operatorname{ker}(q) \rightarrow Z$ is the linear map given by $\tilde{q}(x+\operatorname{ker}(q))=q(x)$, for every $x \in X$. If $X$ and $Z$ are Banach spaces and $q$ is bounded, then $\tilde{q}$ is an isomorphism between $X / \operatorname{ker}(q)$ and $Z$.

More examples of 3SP are the following:

- Separability;

- Given a cardinal $\kappa$, having weight $\kappa$;

- Being isomorphic to $c_{0}$;

- Being isomorphic to $\ell_{\infty}$;

- Being isomorphic to $\ell_{1}$.

A natural question is if being isomorphic to $\ell_{p}, 1<p<\infty$, is a 3 -space property. The case $p=2$, and more generally the question for Hilbert spaces, is known as

Palais' Problem. Is being isomorphic to a Hilbert space a 3SP?

The answer is negative. More generally, being isomorphic to $\ell_{p}$ is not a $3 \mathrm{SP}$, for any $1<p<\infty$.

In the case of Palais' Problem the first counterexample was obtained in (ENFLO; LINDENSTRAUSS; PISIER, 1975). We shall present here the counterexamples obtained by Kalton and Peck in (KALTON; PECK, 1979), which show that being isomorphic to $\ell_{p}$ is not a $3 S P$, $1<p<\infty$. We present the Kalton-Peck spaces since the technique to obtain them through quasilinear maps is standard in the theory of twisted sums of Banach spaces. 


\subsection{Twisted sums and quasilinear maps}

The results of this section are part of the classical theory of twisted sums of Banach spaces, and may be found in (CASTILLO; GONZÁLEZ, 1997).

In order to present the Kalton-Peck spaces as defined in (KALTON, 1978) we must deal with quasi-Banach spaces. A reference for the topic is (KALTON; PECK; ROBERTS, 1984).

Definition 2.2.1. A quasi-norm on a vector space $X$ is a function $\|\|:. X \rightarrow[0, \infty)$ satisfying:

QN1 $\|x\|=0$ if and only if $x=0$;

$Q N 2\|\alpha x\|=|\alpha|\|x\|$, for every $x \in X$, for every $\alpha \in \mathbb{C}$;

QN3 There is a constant $K>0$ such that for all $x, y \in X$ we have

$$
\|x+y\| \leq K(\|x\|+\|y\|)
$$

If $X$ is provided with a quasi-norm, $X$ is said to be a quasi-normed space. Two quasi-norms $\|$. and $\|. \mid\|$ on $X$ are said to be equivalent if there are $c, C>0$ such that for every $x \in X$ we have

$$
c\|x\| \leq\|x\| \leq C\|x\|
$$

Let $p \in(0,1]$. A quasi-norm $\|$.$\| on X$ is said to be $\mathrm{p}$-subadditive if

$$
\left\|x_{1}+x_{2}\right\|^{p} \leq\left\|x_{1}\right\|^{p}+\left\|x_{2}\right\|^{p}
$$

The following result allows us to consider a metric on quasi-normed spaces.

Theorem 2.2.2 (Aoki-Rolewicz Theorem, (AOKI, 1942; ROLEWICZ, 1957)). Let X be a quasi-normed space. Then there is $p \in(0,1]$ and an equivalent quasi-norm $\|$.$\| on X$ such that

$$
\left\|x_{1}+x_{2}\right\|^{p} \leq\left\|x_{1}\right\|^{p}+\left\|x_{2}\right\|^{p}
$$

for every $x_{1}, x_{2} \in X$.

This allows us to consider a metric on quasi-normed spaces:

Definition 2.2.3 (Metric on a quasi-normed space). Let $X$ be a quasi-normed space, take any equivalent $p$-subadditive quasi-norm $\||\|\mid\|$ on $X$ and let

$$
d\left(x_{1}, x_{2}\right)=\left\|x_{1}-x_{2}\right\|^{p}
$$

for every $x_{1}, x_{2} \in X$. If this distance is complete, $X$ is said to be a quasi-Banach space.

Notice that if $\|$.$\| satisfies QN3 with C=1$ then $\|$.$\| is a norm. By an operator between$ quasi-normed spaces we refer to continuous linear maps. As for normed spaces, continuity of a linear map is the same as boundedness. Similarly, we have the notion of isomorphism of quasi-normed spaces. The Open Mapping Theorem holds for quasi-Banach spaces, i. e., if $X$ and $Y$ are quasi-Banach spaces and $T: X \rightarrow Y$ is a surjective operator, then $T$ is an open mapping ((RUDIN, 1991), Theorem 2.11).

Definition 2.2.4. Let $Y$ and $Z$ be Banach spaces. A twisted sum of $Y$ and $Z$ is a quasi-normed space $X$ with a subspace $Y_{0}$ isomorphic to $Y$ such that $X / Y_{0}$ is isomorphic to $Z$.

This may be described by short exact sequence

$$
0 \longrightarrow Y \stackrel{i}{\longrightarrow} X \stackrel{q}{\longrightarrow} Z \longrightarrow 0
$$

where $i$ is an isomorphism onto its image and $q$ induces an isomorphism between $Z$ and $X / i(Y)$.

If $Y=Z$, then we say that $X$ is an extension of $Y$. 
Notice that we do not suppose that $X$ is complete.

Proposition 2.2.5 ((CASTILLO; GONZÁLEZ, 1997), Lemma 1.5.b.). A twisted sum of Banach spaces is a quasi-Banach space, i. e., is complete.

Suppose that we have a short exact sequence (2.1) and we know beforehand that $X$ is complete. Then the Open Mapping theorem implies automatically that $i$ is an isomorphism onto its image and that the quotient map induces an isomorphism.

Given two Banach spaces $Y$ and $Z$ we always have a twisted sum of them by means of the direct sum.

Definition 2.2.6 (Trivial twisted sum). Let $Y$ and $Z$ be Banach spaces. The trivial twisted sum of $Y$ and $Z$ is the direct sum $Y \oplus Z$, which may be described by the short exact sequence

$$
0 \longrightarrow Y \stackrel{i}{\longrightarrow} Y \oplus Z \stackrel{q}{\longrightarrow} Z \longrightarrow 0
$$

where $i: y \mapsto(y, 0)$ and $q:(y, z) \mapsto z$.

Twisted sums are in correspondence with maps called quasilinear.

Definition 2.2.7. Let $Y$ and $Z$ be normed spaces. A map $F: Z \rightarrow Y$ is homogeneous if $F(\lambda z)=$ $\lambda F(z)$ for every $\lambda \in \mathbb{C}$ and $z \in Z$. The map $F$ is quasilinear if $F$ is homogeneous and there is $K>0$ such that for all $z_{1}, z_{2} \in Z$,

$$
\left\|F\left(z_{1}+z_{2}\right)-F\left(z_{1}\right)-F\left(z_{2}\right)\right\| \leq K\left(\left\|z_{1}\right\|+\left\|z_{2}\right\|\right)
$$
examples.

Notice that any linear map $F: Z \rightarrow Y$ is quasilinear. We shall soon give nontrivial

Using a quasilinear map $F: Z \rightarrow Y$ we can define a quasi-norm on $Y \times Z$.

Proposition 2.2.8 ((KALTON; PECK, 1979)). Let $Y$ and $Z$ be normed spaces and $F: Z \rightarrow Y$ be a quasilinear map. Then $\|\cdot\|_{F}$ is a quasinorm on $Y \times Z$, where for all $y \in Y, z \in Z$,

$$
\|(y, z)\|_{F}=\|y-F(z)\|_{Y}+\|z\|_{Z}
$$

Definition 2.2.9. Let $Y$ and $Z$ be normed spaces and $F: Z \rightarrow Y$ be a quasilinear map. The space $Y \oplus_{F} Z$ is the quasi-normed space $\left(Y \times Z,\|.\|_{F}\right)$.

Proposition 2.2.10 ((KALTON; PECK, 1979)). Let $Y$ and $Z$ be Banach spaces, and let $F: Z \rightarrow Y$ be a quasilinear map. Then $Y \oplus_{F} Z$ is a twisted sum of $Y$ and $Z$. More specifically, we have a short exact sequence

$$
0 \longrightarrow Y \stackrel{i}{\longrightarrow} Y \oplus_{F} Z \stackrel{q}{\longrightarrow} Z \longrightarrow 0
$$

where $i: y \mapsto(y, 0)$ and $q:(y, z) \mapsto z$. In particular, $Y \oplus_{F} Z$ is a quasi-Banach space.

Example 2.2.11. Let $F: Z \rightarrow Y$ be a linear map. In that case, $i(Y)$ is complemented in $Y \oplus_{F} Z$. Indeed, let $P: Y \oplus_{F} Z \rightarrow Y \oplus_{F} Z$ be given by $P(y, z)=(y-F(z), 0)$. Then $P$ is linear, $P^{2}=P$, and $\|P\| \leq 1$. Besides, $\operatorname{Im}(P)=i(Y)$.

Notice that $Y \oplus Z=Y \oplus_{0} Z$.

Definition 2.2.12. Let $X_{1}$ and $X_{2}$ be quasi-normed spaces, and let $T: X_{1} \rightarrow X_{2}$. A section for $T$ is a map $s: X_{2} \rightarrow X_{1}$ such that $T \circ s=I d_{X_{2}}$. A retraction for $T$ is a map $r: X_{2} \rightarrow X_{1}$ such that $r \circ T=I d_{X_{1}}$. 
We now describe how to obtain a quasilinear map from a twisted sum.

Definition 2.2.13. Let $H: X \rightarrow Y$ be a homogeneous map between quasi-normed spaces. $\|H\|$ is defined as

$$
\|H\|=\sup _{x \in S_{X}}\|H(x)\| \in[0, \infty]
$$

A homogeneous map $H$ is bounded if $\|H\|<\infty$.

Notice that for every $x \in X$, we have $\|H(x)\| \leq\|H\|\|x\|$.

The following is a consequence of the proof of Theorem 2.4 of (KALTON; PECK, 1979).

Proposition 2.2.14. Consider a twisted sum of Banach spaces described by the short exact sequence

$$
0 \longrightarrow Y \stackrel{i}{\longrightarrow} X \stackrel{q}{\longrightarrow} Z \longrightarrow 0
$$

Let $B: Z \rightarrow X$ be a homogeneous bounded section for $q$ and let $L: Z \rightarrow X$ be a linear section for q. Then $F=i^{-1} \circ(B-L): Z \rightarrow Y$ is a quasilinear map.

Definition 2.2.15. In the situation of Proposition 2.2.14 the quasilinear map $F=i^{-1} \circ(B-L)$ : $Z \rightarrow Y$ will be called a quasilinear map induced by the twisted sum.

The quasilinear map $F$ induces a twisted sum $Y \oplus_{F} Z$, which is equivalent to the original twisted sum in a suitable sense.

Definition 2.2.16. Let $Y$ and $Z$ be Banach spaces, and consider two twisted sums $X_{1}$ and $X_{2}$ of $Y$ and $Z$ described by the short exact sequences

$$
0 \longrightarrow Y \stackrel{i_{j}}{\longrightarrow} X_{j} \stackrel{q_{j}}{\longrightarrow} Z \longrightarrow 0
$$

$j=0,1$. The short exact sequences are equivalent if there is a linear isomorphism $T: X_{1} \rightarrow X_{2}$ making the following diagram commute

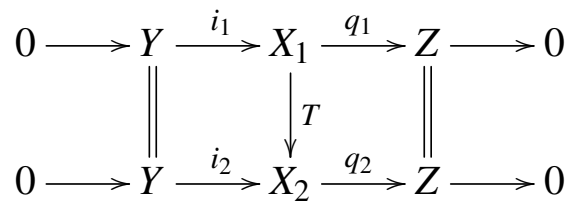

If the equalities in the diagram are instead multiples of the identity then the short exact sequences are said to be projectively equivalent. If instead they are isomorphisms, $X_{1}$ and $X_{2}$ are said to be isomorphically equivalent.

A twisted sum $X$ of $Y$ and $Z$ described by the short exact sequence

$$
0 \longrightarrow Y \stackrel{i}{\longrightarrow} X \stackrel{q}{\longrightarrow} Z \longrightarrow 0
$$

is trivial if $i(Y)$ is complemented in $X$, which is equivalent to (2.2) being equivalent to

$$
0 \longrightarrow Y \longrightarrow Y \oplus Z \longrightarrow Z \longrightarrow 0
$$

The definition of equivalence of short exact sequences translates the concept of $Y$ embedding inside $X_{1}$ and $X_{2}$ in essentially the same way, and defines an equivalence relation on the set of twisted sums of $Y$ and $Z$.

The following lemma is a version of the five lemma from homological algebra, and may be found in (CASTILLO; GONZÁLEZ, 1997), page 3. 
Lemma 2.2.17 (3-Lemma). Suppose we have a commutative diagram

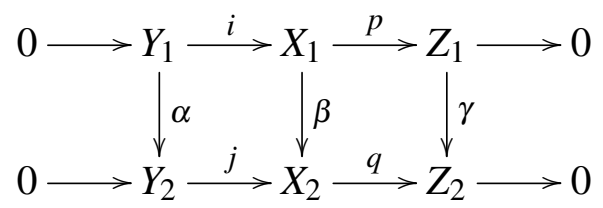

where the lines are exact, the spaces are quasi-Banach, the maps are linear and $i, p, j$ and $q$ are bounded.

1. If $\alpha$ and $\gamma$ are injective, so is $\beta$.

2. If $\alpha$ and $\gamma$ are surjective, so is $\beta$.

3. If $\alpha$ and $\gamma$ are bijective and $\beta$ is bounded, then $\beta$ is an isomorphism between $X_{1}$ and $X_{2}$.

Theorem 2.2.18 ((KALTON; PECK, 1979), Theorem 2.4). Let

$$
0 \longrightarrow Y \stackrel{i}{\longrightarrow} X \stackrel{q}{\longrightarrow} Z \longrightarrow 0
$$

be a short exact sequence and $F: Z \rightarrow Y$ a quasilinear map induced by $X$. Then $X$ and $Y \oplus_{F} Z$ are equivalent.

Equivalence of twisted sums may be translated into an equivalence relation of quasilinear maps.

Definition 2.2.19. Let $Y$ and $Z$ be Banach spaces. Two quasilinear maps $F, G: Z \rightarrow Y$ are equivalent if the short exact sequence corresponding to $Y \oplus_{F} Z$ is equivalent to the one corresponding to $Y \oplus_{G} Z$. If $F$ is equivalent to 0 (or equivalently, if $Y \oplus_{F} Z$ is trivial), it is called trivial.

Proposition 2.2.20 ((KALTON; PECK, 1979), Theorem 2.5). Let $Y$ and $Z$ be Banach spaces, and let $F, G: Z \rightarrow Y$ be quasilinear maps. Then $F$ is equivalent to $G$ if and only if there is a linear map $A: Z \rightarrow Y$ such that $\|F-G+A\|<\infty$.

In general the direct sum will not show that a given property is not a $3 \mathrm{SP}$, so we seek twisted sums which are nontrivial.

The following is a criterion for triviality of a twisted sum.

Proposition 2.2.21 ((CASTILLO; GONZÁLEZ, 1997), Lemma 1.1.a). Consider a twisted sum of Banach spaces

$$
0 \longrightarrow Y \stackrel{i}{\longrightarrow} X \stackrel{q}{\longrightarrow} Z \longrightarrow 0
$$

The following are equivalent:

1. $X$ is trivial;

2. q admits a bounded linear section;

3. $i$ admits a bounded linear retraction.

We aim to present the Kalton-Peck spaces, which are defined by means of quasilinear maps $\mathscr{K}_{p}: \ell_{p} \rightarrow \ell_{p}, 1<p<\infty$. As in many cases when we work with the spaces $\ell_{p}$, it is enough to consider vectors of finite support. The next proposition clarifies this point.

Proposition 2.2.22 ((KALTON; PECK, 1979), Theorem 3.1). Let $Y$ and $Z$ be Banach spaces, and let $Z_{0}$ be a dense linear subspace of $Z$. Suppose that $F_{0}: Z_{0} \rightarrow Y$ is a quasilinear map. Then, up to equivalence of quasilinear maps, $F_{0}$ admits a unique extension $F: Z \rightarrow Y$. 
Definition 2.2.23. Let $1<p<\infty$ and let $\left(e_{n}\right)$ be the standard basis of $\ell_{p}$. The Kalton-Peck map $\mathscr{K}_{p}: \ell_{p} \rightarrow \ell_{p}$ is the quasilinear map which on finitely supported vectors $x=\sum x_{i} e_{i}$ is given by

$$
\mathscr{K}_{p}(x)=\sum x_{i} \log \frac{\left|x_{i}\right|}{\|x\|_{p}} e_{i}
$$

where $r \log s=0$ if $r=0$. The Kalton-Peck space $Z_{p}$ is the twisted sum $\ell_{p} \oplus \mathscr{K}_{p} \ell_{p}$.

In (KALTON; PECK, 1979), Kalton and Peck show that $\mathscr{K}_{p}$ is indeed a quasilinear map and also:

Theorem 2.2.24 ((KALTON; PECK, 1979)). For each $p \in(1, \infty)$, the Kalton-Peck space $Z_{p}$ is a nontrivial extension of $\ell_{p}$.

It is not by chance that these maps appear. They reveal an important connection with interpolation theory, as the next section shows.

Kalton-Peck's space satisfy an important property: the quotient map $q: Z_{p} \rightarrow \ell_{p}$ is strictly singular (it is never an isomorphism when restricted to an infinite dimensional closed subspace), and the inclusion $i: \ell_{p} \rightarrow Z_{p}$ is strictly cosingular (the composition $T \circ i$ is never surjective when the range of $T$ is infinite dimensional).

Definition 2.2.25. Consider a short exact sequence

$$
0 \longrightarrow Y \stackrel{i}{\longrightarrow} X \stackrel{q}{\longrightarrow} Z \longrightarrow 0
$$

Both the short exact sequence and the twisted sum $X$ are called singular if $q$ is strictly singular, and cosingular if $i$ is strictly cosingular. If $F$ is a quasilinear map associated to the twisted sum, it is called singular or cosingular accordingly.

It follows from the definitions that $F: Z \rightarrow Y$ is singular if and only if $\left.F\right|_{W}$ is not trivial whenever $W \subset Z$ is a closed infinite dimensional subspace.

\subsection{Complex Interpolation}

\subsubsection{Complex Interpolation of Couples}

The origins of interpolation theory remount to the Riesz-Thorin Theorem.

Theorem 2.3.1 (Riesz-Thorin Theorem, (RIESZ, 1926; THORIN, 1938)). Let $T: \ell_{\infty} \rightarrow \ell_{\infty}$ be an operator such that $T\left(\ell_{1}\right) \subset \ell_{1}$ and $T$ is bounded as a linear map from $\ell_{1}$ into $\ell_{1}$. Then $T\left(\ell_{p}\right) \subset \ell_{p}$ and

$$
\left\|T: \ell_{p} \rightarrow \ell_{p}\right\| \leq\left\|T: \ell_{\infty} \rightarrow \ell_{\infty}\right\|^{1-\theta}\left\|T: \ell_{1} \rightarrow \ell_{1}\right\|^{\theta}
$$

where $\theta=\frac{1}{p}$, for every $1<p<\infty$.

The motivation for interpolation theory is finding families of spaces that behave like the $\ell_{p}$ spaces in the Riesz-Thorin Theorem.

We now present the complex interpolation method which is due to Calderón and independently to Lions (LIONS, 1960; CALDERÓN, 1964). We indicate the reader to (BERGH; LÖFSTRÖM, 1976) for details. 
Definition 2.3.2. A couple of Banach spaces $\bar{X}=\left(X_{0}, X_{1}\right)$ is called compatible if there are continuous linear injections $i_{j}: X_{j} \rightarrow \mathscr{U}, j=0,1$, into a topological Hausdorff vector space $\mathscr{U}$. In that case, we identify $X_{0}$ and $X_{1}$ with their images $i_{0}\left(X_{0}\right)$ and $i_{1}\left(X_{1}\right)$.

The sum space of the compatible couple $\bar{X}$ is the space

$$
\Sigma(\bar{X})=\left\{x_{0}+x_{1}: x_{0} \in X_{0}, x_{1} \in X_{1}\right\}
$$

with the norm

$$
\|x\|=\inf \left\{\left\|x_{0}\right\|_{X_{0}}+\left\|x_{1}\right\|_{X_{1}}: x=x_{0}+x_{1}, x_{0} \in X_{0}, x_{1} \in X_{1}\right\}
$$

The intersection space of the compatible couple $\bar{X}$ is the space

$$
\Delta(\bar{X})=X_{0} \cap X_{1}
$$

with the norm

$$
\|x\|=\max \left\{\|x\|_{X_{0}},\|x\|_{X_{1}}\right\}
$$

Observation 2.3.3. Both spaces $\sum(\bar{X})$ and $\Delta(\bar{X})$ are complete (see (BERGH; LÖFSTRÖM, 1976), Lemma 2.3.1).

Notation 2.3.4. $\mathbb{S}=\{z \in \mathbb{C}: 0<\operatorname{Re}(z)<1\}$

Notation 2.3.5. $\mathbb{S}_{j}=\{z \in \mathbb{C}: \operatorname{Re}(z)=j\}, j=0,1$

Definition 2.3.6. Let $\bar{X}=\left(X_{0}, X_{1}\right)$ be a compatible couple of Banach spaces. The space $\mathscr{F}=$ $\mathscr{F}(\bar{X})$ is defined as the space of all functions $f: \overline{\mathbb{S}} \rightarrow \Sigma(\bar{X})$ such that:

1. $f$ is continuous and bounded on $\overline{\mathbb{S}}$ and analytic on $\mathbb{S}$;

2. $\left.f\right|_{\mathbb{S}_{j}}: \mathbb{S}_{j} \rightarrow X_{j}$ is continuous, $j=0,1$;

3. The functions $t \mapsto\|f(j+i t)\|_{X_{j}}$ go to 0 as $|t| \rightarrow \infty, j=0,1$.

The space $\mathscr{F}$ is equipped with the complete norm

$$
\|f\|=\max \left\{\sup _{t \in \mathbb{R}}\|f(i t)\|_{X_{0}}, \sup _{t \in \mathbb{R}}\|f(1+i t)\|_{X_{1}}\right\}
$$

Observation 2.3.7. For $z \in \mathbb{S}$, let $\delta_{z}: \mathscr{F} \rightarrow \Sigma(\bar{X})$ be given by $\delta_{z}(f)=f(z)$. Then $\delta_{z}$ is a bounded operator.

Definition 2.3.8. For $z \in \mathbb{S}$, the interpolation space $X_{z}$ is given by $X_{z}=\mathscr{F} / \operatorname{ker}\left(\delta_{z}\right)$.

That is,

$$
X_{z}=\{f(z): f \in \mathscr{F}\}
$$

with the quotient norm

$$
\|x\|_{z}=\inf \{\|f\|: f \in \mathscr{F}, f(z)=x\}
$$

Notice that for every $t \in \mathbb{R}$ and every $f \in \mathscr{F}$ we have $\|f\|=\|g\|$, where $g$ is given by $g(z)=f(z+i t)$. Therefore, it is enough to work with $z \in(0,1)$.

Example 2.3.9. The couple $\left(\ell_{\infty}, \ell_{1}\right)$ is a compatible couple $\left(\ell_{1} \subset \ell_{\infty}\right)$, and for every $\theta \in(0,1)$ we have $\left(\ell_{\infty}, \ell_{1}\right)_{\theta}=\ell_{p}$, where $p=\frac{1}{\theta}$. 
The complex method satisfies the Riesz-Thorin Theorem. Actually, it is based on the proof of the Riesz-Thorin Theorem.

Theorem 2.3.10. [(CALDERÓN, 1964), §7] Let $\bar{X}=\left(X_{0}, X_{1}\right)$ and $\bar{Y}=\left(Y_{0}, Y_{1}\right)$ be compatible couples of Banach spaces. Suppose that $T: \Sigma(\bar{X}) \rightarrow \Sigma(\bar{Y})$ is a linear map such that $T: X_{0} \rightarrow Y_{0}$ and $T: X_{1} \rightarrow Y_{1}$ are operators. Then for every $0<\theta<1$ we have $T\left(X_{\theta}\right) \subset Y_{\theta}$ and

$$
\left\|T: X_{\theta} \rightarrow Y_{\theta}\right\| \leq\left\|T: X_{0} \rightarrow Y_{0}\right\|^{1-\theta}\left\|T: X_{1} \rightarrow Y_{1}\right\|^{\theta}
$$

Proof. Denote $M_{i}=\left\|T: X_{i} \rightarrow Y_{i}\right\|, i=0,1$. We begin by showing that $T: \Sigma(\bar{X}) \rightarrow \Sigma(\bar{Y})$ is bounded. Let $x \in \Sigma(\bar{X})$ and take $x_{i} \in X_{i}, i=0,1$, such that $x=x_{0}+x_{1}$. Then

$$
T(x)=T\left(x_{0}\right)+T\left(x_{1}\right)
$$

and $T\left(x_{i}\right) \in Y_{i}$. So

$$
\|T(x)\|_{\Sigma(Y)} \leq\left\|T\left(x_{0}\right)\right\|_{Y_{0}}+\left\|T\left(x_{1}\right)\right\|_{Y_{1}} \leq \max \left\{M_{0}, M_{1}\right\}\left(\left\|x_{0}\right\|_{X_{0}}+\left\|x_{1}\right\|_{X_{1}}\right)
$$

so that $\|T: \Sigma(\bar{X}) \rightarrow \Sigma(\bar{Y})\| \leq \max \left\{M_{0}, M_{1}\right\}$.

Let $x \in X_{\theta}$, and take $f \in \mathscr{F}$ such that $f(\theta)=x$. Define $g: \mathbb{S} \rightarrow \Sigma(\bar{Y})$ by

$$
g(z)=M_{0}^{-1+z} M_{1}^{-z} T(f(z))
$$

Since $T: \Sigma(\bar{X}) \rightarrow \Sigma(\bar{Y})$ is bounded, $g \in \mathscr{F}(\bar{Y})$, and

$$
\|g\| \leq\|f\|
$$

But $g(\theta)=M_{0}^{-1+\theta} M_{1}^{-\theta} T(x)$, so that

$$
\|T(x)\|_{Y_{\theta}} \leq M_{0}^{1-\theta} M_{1}^{\theta}\|x\|_{X_{\theta}}
$$

The next proposition shows that it is enough to consider a certain class of well behaved functions in most cases, and also that $\Delta(\bar{X})$ is dense in each space $X_{\theta}, 0<\theta<1$. We have reformulated it according to our needs.

Proposition 2.3.11 ((CALDERÓN, 1964), §9.2). Let $\bar{X}=\left(X_{0}, X_{1}\right)$ be a compatible couple, and let $\mathscr{F}_{0} \subset \mathscr{F}$ be the subspace generated by the functions of the form

$$
g(z)=\sum_{i=1}^{n} \varphi_{i}(z) x_{i}
$$

such that $\varphi_{i}$ is an analytic function, $x_{i} \in \Delta(\bar{X}), i=1, \ldots, n$, and $g \in \mathscr{F}$. Then $\mathscr{F}_{0}$ is dense in $\mathscr{F}$. Consequently, $\Delta(\bar{X})$ is dense in $X_{\theta}, 0<\theta<1$.

The connection with twisted sums is given by the following simple question: we are working with analytic functions, so what about their derivative? The next proposition may be essentially found in (CARRO; CERDÁ; SORIA, 1995), Theorem 4.1.

Proposition 2.3.12. For $\theta \in(0,1)$, let $\delta_{\theta}^{\prime}: \operatorname{ker} \delta_{\theta} \rightarrow X_{\theta}$ be given by $\delta_{\theta}^{\prime}(f)=f^{\prime}(\theta)$. Then $\delta_{\theta}^{\prime}$ is a surjective bounded operator. 
Proof. Let $\mathbb{D}$ be the open unitary disk in $\mathbb{C}$ and $\varphi: \mathbb{S} \rightarrow \mathbb{D}$ be a conformal equivalence, i. e., a bijective holomorphic function. Notice that $\varphi$ is also defined continuously on $\overline{\mathbb{S}}$, and $\varphi(\partial \mathbb{S}) \subset \partial \mathbb{D}$.

Let $f \in \mathscr{F}$ with $f(\theta)=0$. Then the function $g=\varphi^{\prime}(\theta) \frac{f}{\varphi}: \mathbb{S} \rightarrow \Sigma(\bar{X})$ given by

$$
g(z)= \begin{cases}\varphi^{\prime}(\theta) \frac{f(z)}{\varphi(z)} & \text { if } z \neq \theta \\ f^{\prime}(\theta) & \text { if } z=\theta\end{cases}
$$

is in $\mathscr{F}$ and $\|g\|=\left|\varphi^{\prime}(\theta)\right|\|f\|$. So $f^{\prime}(\theta) \in X_{\theta}$ and

$$
\left\|f^{\prime}(\theta)\right\|_{X_{\theta}} \leq\|g\|=\left|\varphi^{\prime}(\theta)\right|\|f\|
$$

so that $\left\|\delta_{\theta}^{\prime}\right\| \leq\left|\varphi^{\prime}(\theta)\right|$.

To check that it is surjective, take $x \in X_{\theta}$ and $f \in \mathscr{F}$ such that $f(\theta)=x$. Then $g=$ $\frac{\varphi}{\varphi^{\prime}(\theta)} f \in \operatorname{ker}\left(\delta_{\theta}\right) \subset \mathscr{F}$ and $g^{\prime}(\theta)=x$.

The next proposition is a classical construction in homology theory. We refer to (CASTILLO; GONZÁLEZ, 1997) for the second part.

Proposition 2.3.13. Suppose we have a diagram of Banach spaces and operators

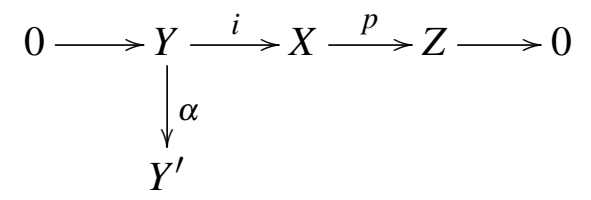

Then there is a Banach space $\mathscr{P} \mathscr{O}$, called the pushout of the diagram, and operators $j: Y^{\prime} \rightarrow \mathscr{P} \mathscr{O}, T: X \rightarrow \mathscr{P} \mathscr{O}$ and $q: \mathscr{P} \mathscr{O} \rightarrow Z$ making the following diagram commute:

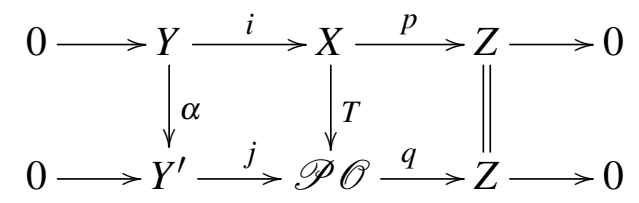

Also, if $F$ is a quasilinear map defining $X$, then $\alpha \circ F$ is a quasilinear map defining the pushout exact sequence.

Proof. The space $\mathscr{P} \mathscr{O}$ is defined the following way: let

$$
\Delta=\{(i(y),-\alpha(y)): y \in Y\} \subset X \oplus_{\infty} Y^{\prime}
$$

Then $\Delta$ is closed in $X \oplus_{\infty} Y^{\prime}, \mathscr{P} \mathscr{O}=X \oplus_{\infty} Y^{\prime} / \Delta, j\left(y^{\prime}\right)=\left(0, y^{\prime}\right)+\Delta, T(x)=(x, 0)+\Delta$ and $q\left(\left(x, y^{\prime}\right)+\Delta\right)=p(x)$.

We prove only the second part, regarding the quasilinear map $\alpha \circ F$. In this text, the first one may be found in Chapter 5 in the context of operator spaces.

Let $B: Z \rightarrow X$ be a bounded homogeneous section for $p$, and $L: Z \rightarrow X$ be a linear section for $p$. Then $F=i^{-1} \circ(B-L)$ is a quasilinear map defining $X$.

Then $T B: Z \rightarrow \mathscr{P} \mathscr{O}$ is a bounded homogeneous section for $q$, and $T L: Z \rightarrow \mathscr{P} \mathscr{O}$ is a linear section for $q$. Therefore, $G=j^{-1} \circ T(B-L): Z \rightarrow Y^{\prime}$ is a quasilinear map defining $\mathscr{P} \mathscr{O}$.

Then

$$
G=j^{-1} \circ T \circ i \circ i^{-1} \circ(B-L)=\alpha \circ F
$$


We are now ready to tell the connection between complex interpolation and twisted sums of Banach spaces. By Proposition 2.3.12, we have the following diagram:

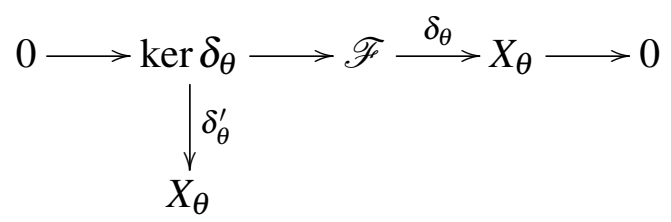

which by Proposition 2.3.13 may be completed into a commutative diagram

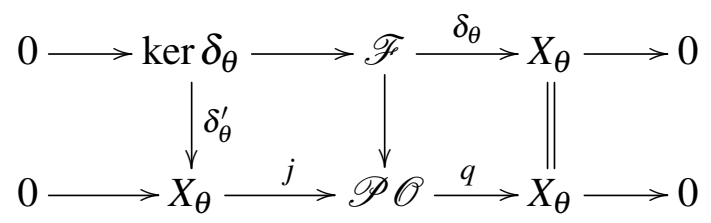

That is, we get an extension of the interpolation space $X_{\theta}$. Notice that $\mathscr{P} \mathscr{O}$ is a Banach space, so the problem of its local convexity is automatically solved.

Proposition 2.3.13 tells us how to get the quasilinear map defining $\mathscr{P} \mathscr{O}$ : take a bounded homogeneous section $B_{\theta}: X_{\theta} \rightarrow \mathscr{F}$ for $\delta_{\theta}$ and a linear section $L_{\theta}: X_{\theta} \rightarrow \mathscr{F}$ for $\delta_{\theta}$. Then $\omega_{\theta}=\delta_{\theta}^{\prime} \circ\left(B_{\theta}-L_{\theta}\right)$ is a quasilinear map defining the pushout exact sequence.

This twisted sum also has the following alternative descriptions:

Proposition 2.3.14 ((KALTON; MONTGOMERY-SMITH, 2003)). Each of the following short exact sequences is equivalent to the pushout exact sequence induced by complex interpolation:

1.

$$
0 \longrightarrow X_{\theta} \longrightarrow \mathscr{F} /\left(\operatorname{ker}\left(\delta_{\theta}\right) \cap \operatorname{ker}\left(\delta_{\theta}^{\prime}\right)\right) \longrightarrow X_{\theta} \longrightarrow 0
$$

where we identify $\mathscr{F} /\left(\operatorname{ker}\left(\boldsymbol{\delta}_{\theta}\right) \cap \operatorname{ker}\left(\delta_{\theta}^{\prime}\right)\right)$ with the space

$$
\left.\left\{f(\theta), f^{\prime}(\theta)\right): f \in \mathscr{F}\right\}
$$

2.

with the quotient norm. The inclusion is $y \mapsto(0, y)$ and the quotient is $(x, y) \mapsto x$.

$$
0 \longrightarrow X_{\theta} \longrightarrow d_{\Omega_{\theta}} X_{\theta} \longrightarrow X_{\theta} \longrightarrow 0
$$

where, if $B_{\theta}$ is a bounded homogeneous section for $\delta_{\theta}, \Omega_{\theta}=\delta_{\theta}^{\prime} B_{\theta}: X_{\theta} \rightarrow \Sigma(\bar{X})$,

$$
d_{\Omega_{\theta}} X_{\theta}=\left\{(x, y) \in \Sigma(\bar{X}) \times X_{\theta}: x-\Omega_{\theta} y \in X_{\theta}\right\}
$$

with the quasinorm $\|(x, y)\|=\left\|x-\Omega_{\theta} y\right\|_{\theta}+\|y\|_{\theta}$, the inclusion is $x \mapsto(x, 0)$ and the quotient map is $(x, y) \mapsto y$.

The map $\Omega_{\theta}$ depends on the choice of $B_{\theta}$, but if $C_{\theta}$ is another homogeneous bounded section for $\delta_{\theta}$ we have for any $x \in X_{\theta}$ that $B_{\theta}(x)-C_{\theta}(x) \in \operatorname{ker}\left(\delta_{\theta}\right)$, and therefore $\delta_{\theta}^{\prime}\left(B_{\theta}(x)-\right.$ $\left.C_{\theta}(x)\right) \in X_{\theta}$ and

$$
\left\|\Omega_{\theta}(x)-\delta_{\theta}^{\prime} C_{\theta}(x)\right\|_{X_{\theta}} \leq\left\|\delta_{\theta}^{\prime}\right\|\left(\left\|B_{\theta}\right\|+\left\|C_{\theta}\right\|\right)\|x\|
$$

i. e., $\Omega_{\theta}$ is equivalent to $\delta_{\theta}^{\prime} C_{\theta}$. When no confusion may arise, we will simply denote the space $d_{\Omega_{\theta}} X_{\theta}$ by $d X_{\theta}$.

As much as it looks like, $\Omega_{\theta}$ is not what we called a quasilinear map defining the short exact sequence

$$
0 \longrightarrow X_{\theta} \longrightarrow d_{\Omega_{\theta}} X_{\theta} \longrightarrow X_{\theta} \longrightarrow 0
$$

Indeed, its image is not in $X_{\theta}$. This justifies the following definition. 
Definition 2.3.15. We will say that $\Omega_{\theta}$ is trivial if the natural copy of $X_{\theta}$ is complemented in $d X_{\theta}$. We shall also say that $\Omega_{\theta}$ is singular if the natural quotient map $d X_{\theta} \rightarrow X_{\theta}$ is strictly singular.

Observation 2.3.16. Condition 3. of Definition 2.3.6 may be replaced by

3' The functions $t \mapsto\|f(j+i t)\|_{X_{j}}$ are bounded, $j=0,1$.

and the resulting interpolation and derived spaces are not changed. To check this, multiply a function satisfying 3' by $e^{\delta(z-\theta)^{2}}$ for $\delta>0$ and let $\delta \rightarrow 0$.

Example 2.3.17. Consider the compatible couple $\left(\ell_{\infty}, \ell_{1}\right)$, let $p \in(1, \infty)$ and $x \in c_{00}$. Then, the function $f(z)=\sum\left(\frac{\left|x_{i}\right|}{\|x\|_{p}}\right)^{z p} \frac{x_{i}}{\left|x_{i}\right|} e_{i}$, is in $\mathscr{F},\|f\|=\|x\|_{\ell_{p}}$, and for $\theta=\frac{1}{p}$ we have $f(\theta)=x$.

Then $B_{\theta}(x)=f$ defines a bounded homogeneous section from $c_{00} \subset \ell_{p}$ into $\mathscr{F}$ for $\delta_{\theta}$, and one verifies that $\Omega_{\theta}=p \mathscr{K}_{p}$, a multiple of the Kalton-Peck map.

This shows that the Kalton-Peck map is quasilinear, and that $Z_{p}$ is isomorphic to a Banach space.

We end this subsection with a discussion of reiteration: for certain results it is more convenient to work not with the original interpolation couple $\bar{X}=\left(X_{0}, X_{1}\right)$, but with a couple that comes from $\bar{X}$. The next proposition regards the stability of this construction. The first part may be found in (BERGH; LÖFSTRÖM, 1976) as Theorem 4.6.1, and the second is a simple application of the 3-lemma 2.2.17.

Theorem 2.3.18 (Reiteration Theorem). Let $\bar{X}=\left(X_{0}, X_{1}\right)$ be a compatible couple, let $\theta_{j} \in[0,1]$, $j=0,1$. and let $Y_{j}=X_{\theta_{j}}$. Consider the compatible couple $\bar{Y}=\left(Y_{0}, Y_{1}\right)$ (with containing space $\Sigma(\bar{X})$ ). If $\Delta(\bar{Y})$ is dense in $Y_{0}, Y_{1}$ and $\Delta(\bar{X})$, then for every $\theta \in(0,1)$ we have

$$
Y_{\theta}=X_{\eta}
$$

with equality of norms, where $\eta=(1-\theta) \theta_{0}+\theta \theta_{1}$.

Furthermore, $d Y_{\theta}$ and $d X_{\eta}$ are projectively equivalent.

The hypothesis of Theorem 2.3.18 is satisfied if $X_{0} \subset X_{1}$ or $X_{1} \subset X_{0}$ continuously.

\subsubsection{Complex Interpolation of Families}

The theory of complex interpolation of couples may be generalized to interpolating families of Banach spaces. We present here a modification of the interpolation method of (COIFMAN et al., 1982). The modification is minor, so most of the original results are still valid. Since we modified the method, we give full proofs for most results, but we point out that these are straightforward adaptations of the proofs in (COIFMAN et al., 1982).

We modified the original method since it does not allow freedom with respect to the intersection space, i. e., given an interpolation family we must calculate its so called logintersection space, which is by no means an easy task. However, in must cases there is a natural space that should play the role of the intersection space. For example, if we are interpolating a family of spaces with a common Schauder basis then the space of finitely supported vectors is a natural choice of intersection space.

We begin the presentation by considering interpolation on $\mathbb{D}$, and later show how the method extends to more general domains, like the strip. 
Notation 2.3.19. $\mathbb{D}=\{z \in \mathbb{C}:|z|<1\}$

$\mathbb{T}=\partial \mathbb{D}$

On $\mathbb{T}$ we denote $d z=\frac{1}{2 \pi} d \lambda$, where $\lambda$ is the Lebesgue measure on $\mathbb{T}$.

Definition 2.3.20. Let $z_{0}=r e^{i \theta} \in \mathbb{D}$. The Poisson kernel on $\mathbb{T}$ with respect to $z_{0}$ is the function $P_{z_{0}}: \mathbb{T} \rightarrow \mathbb{R}$ given by

$$
P_{z_{0}}\left(e^{i t}\right)=\frac{1-r^{2}}{1+r^{2}-2 r \cos (\theta-t)}
$$

The conjugate Poisson kernel on $\mathbb{T}$ with respect to $z_{0}$ is the function $\tilde{P}_{z_{0}}: \mathbb{T} \rightarrow \mathbb{R}$ given by

$$
\tilde{P}_{z_{0}}\left(e^{i t}\right)=\frac{2 r \sin (\theta-t)}{1+r^{2}-2 r \cos (\theta-t)}
$$

The following result is classical in the theory of harmonic functions. We refer to the book (MASHREGHI, 2009) for an exposition of representation of functions through the Poisson kernel and its conjugate.

Theorem 2.3.21. [Poisson kernel representation] Let $f: \mathbb{T} \rightarrow \mathbb{R}$ be an integrable function with respect to dz. Then there is a unique harmonic function $u: \mathbb{D} \rightarrow \mathbb{R}$ with a. e. nontangential limits on $\mathbb{T}$ which agree with $f$. The function $u$ is given by

$$
u(z)=\int_{0}^{2 \pi} f(z) P_{z_{0}}(z) d z
$$

The harmonic conjugate $\tilde{u}$ of $u$ with $\tilde{u}\left(z_{0}\right)=0$ is given by

$$
\tilde{u}(z)=\int_{0}^{2 \pi} f(z) \tilde{P}_{z_{0}}(z) d z
$$

and also has a. e. nontangential limits on $\mathbb{T}$.

If $f$ is continuous, so is $u$ on $\overline{\mathbb{D}}$.

Notation 2.3.22. $\log ^{+} x=\max \{0, \log x\}$

Definition 2.3.23. Let $\left\{X_{z}: z \in \mathbb{T}\right\}$ be a family of Banach spaces. Suppose we have a Banach space $\mathscr{U}$ and linear continuous injections $i_{z}: X_{z} \rightarrow \mathscr{U}$. From now on we identify $X_{z}$ with its image $i_{z}\left(X_{z}\right) \subset \mathscr{U}$.

Fix a subspace $X$ of $\cap_{z \in \mathbb{T}} X_{z}$. The family $\left\{X_{z}: z \in \mathbb{T} ; X\right\}$ is an interpolation family if, for some $z_{0} \in \mathbb{D}$, we have:

I1 For every $x \in X$ the function $t \mapsto\|x\|_{e^{i t}}$ is measurable and

$$
\int_{0}^{2 \pi} \log ^{+}\|x\|_{e^{i t}} P_{z_{0}}\left(e^{i t}\right) d t<\infty
$$

I2 There is a (fixed) measurable positive function $k$ on $\mathbb{T}$ such that

$$
\int_{0}^{2 \pi} \log ^{+} k\left(e^{i t}\right) P_{z_{0}}\left(e^{i t}\right) d t<\infty
$$

and, for every $z \in \mathbb{T}$ and every $x \in X$

$$
\|x\|_{\mathscr{U}} \leq k(z)\|x\|_{z}
$$


The space $\mathscr{U}$ is called the containing space for the family, $X$ is called the $\Delta$ space of the family, and $k$ is called a containing function for the family.

Observation 2.3.24. It follows from the definition and the properties of the Poisson kernel that these conditions are satisfied for every $z_{0} \in \mathbb{D}$. Indeed, integrability with respect to the Poisson kernel is equivalent to integrability with respect to Lebesgue measure.

We call attention to the fact that in (COIFMAN et al., 1982) it is supposed that for every $x \in \cap_{z \in \mathbb{T}} X_{z}$ the function $t \mapsto\|x\|_{e^{i t}}$ is measurable, and the space $X$ is always the biggest subspace of $\cap_{z \in \mathbb{T}} X_{z}$ satisfying I1, called the log-intersection space.

Example 2.3.25. Let $\partial_{0}$ and $\partial_{1}$ be two disjoint arches of $\mathbb{T}$ such that $\mathbb{T}=\partial_{0} \cup \partial_{1}$.

Let $\bar{X}=\left(X_{0}, X_{1}\right)$ be a compatible couple of Banach spaces in the sense of complex interpolation, and let

- $X_{z}=X_{i}$ for $z \in \partial_{i}, i=0,1$;

- $X=\Delta(\bar{X})=X_{0} \cap X_{1} \subset \Sigma(\bar{X})$

Then $\left\{X_{z}: z \in \mathbb{T} ; X\right\}$ is an interpolation family with containing space $\Sigma(\bar{X})$ and containing function $k \equiv 1$.

The method of complex interpolation for families is a generalization of the complex method for couples. So one must define a space of analytic functions associated to the interpolation family, as in Definition 2.3.6. However, there is a difficulty with condition 2. of Definition 2.3.6: what should be the analogue of $\left.f\right|_{\mathbb{S}_{j}}: \mathbb{S}_{j} \rightarrow X_{j}$ being continuous for $j=0,1$ ?

One way to deal with this difficulty is instead of directly defining the analogue of space $\mathscr{F}$ for the interpolation family to define the space corresponding to the space $\mathscr{F}_{0}$ of Proposition 2.3.11. The functions that will play the role of the exponential functions of Proposition 2.3.11 are the Smirnov functions. A reference for the study of the Nevanlinna and Smirnov classes is (DUREN, 2000).

Definition 2.3.26 (Nevanlinna Class). The Nevanlinna class $N$ is the class of analytic functions $f: \mathbb{D} \rightarrow \mathbb{C}$ such that the integrals

$$
\int_{0}^{2 \pi} \log ^{+}\left|f\left(r e^{i t}\right)\right| d t
$$

are uniformly bounded for $r<1$.

Observation 2.3.27. A function $f: \mathbb{D} \rightarrow \mathbb{C}$ is in $N$ if and only if the integrals

$$
\int_{0}^{2 \pi}\left(1+\log \left|f\left(r e^{i t}\right)\right|\right) d t
$$

are uniformly bounded for $r<1$.

Example 2.3.28. The Hardy classes $H^{p}, 0<p \leq \infty$, are contained in $N$. We recall that $H^{p}$ is the space of functions $f: \mathbb{D} \rightarrow \mathbb{C}$ which are analytic and

$$
\sup _{0 \leq r<1} \int_{0}^{2 \pi}\left|f\left(r e^{i t}\right)\right|^{p} d t<\infty
$$

for $0<p<\infty$, and $H^{\infty}$ is the class of bounded analytic functions on $\mathbb{D}$. 
Proposition 2.3.29 ((DUREN, 2000), Theorem 2.2). Let $f \in N$. Then the nontangential limits $f\left(e^{i t}\right)$ exist for almost every $t \in[0,2 \pi]$, and the function $t \mapsto \log ^{+}\left|f\left(e^{i t}\right)\right|$ is integrable, unless $f \equiv 0$.

Definition 2.3.30 (Smirnov Class). The Smirnov class $N^{+}$is the class of analytic functions $f \in N$ for which

$$
\lim _{r \rightarrow 1} \int_{0}^{2 \pi} \log ^{+}\left|f\left(r e^{i t}\right)\right| d t=\int_{0}^{2 \pi} \log ^{+}\left|f\left(e^{i t}\right)\right| d t
$$

Observation 2.3.31. A function $f: \mathbb{D} \rightarrow \mathbb{C}$ is in $N^{+}$if and only if

$$
\lim _{r \rightarrow 1} \int_{0}^{2 \pi}\left(1+\log \left|f\left(r e^{i t}\right)\right|\right) d t=\int_{0}^{2 \pi}\left(1+\log \left|f\left(e^{i t}\right)\right|\right) d t
$$

Example 2.3.32. The Hardy classes $H^{p}, 0<p \leq \infty$, are contained in $N^{+}$.

Definition 2.3.33. Let $\left\{X_{z}: z \in \mathbb{T} ; X\right\}$ be an interpolation family with containing space $\mathscr{U}$. The space $\mathscr{G}$ is defined as the space of analytic functions $f: \mathbb{D} \rightarrow \mathscr{U}$ of the form

$$
\sum_{i=1}^{n} \psi_{i}(z) x_{i}
$$

such that $\psi_{i} \in N^{+}, x_{i} \in X$, and

$$
\|f\|_{\mathscr{G}}=\underset{z \in \mathbb{T}}{\operatorname{esssup}}\left\|\sum_{i=1}^{n} \psi_{i}(z) x_{i}\right\|_{z}<\infty
$$

We denote by $\mathscr{F}$ the completion of the space $\mathscr{G}$ with respect to $\|.\| \mathscr{G}$.

Observation 2.3.34. To see that for $g \in \mathscr{G}$ the function $z \mapsto\|g(z)\|_{z}, z \in \mathbb{T}$, is measurable, notice that $z \mapsto g(z)$ is $\mathscr{U}$-measurable, and therefore we may approximate it by simple functions, say, $g_{n}$, with image contained in the span of the image of $g$. It is clear that $z \mapsto\left\|g_{n}(z)\right\|_{z}$ is measurable, by I1. Now, for a. e. $z \in \mathbb{T}$ we have $\|g(z)\|_{z}=\lim _{n}\left\|g_{n}(z)\right\|_{z}$, and therefore $z \mapsto\|g(z)\|_{z}$ is measurable.

Observation 2.3.35. Notice that we also use $\mathscr{F}$ to refer to the space of Definition 2.3.6. Usually it will be clear from the context which space we are referring to. If one may be led to confusion, we will change notation accordingly.

It will be useful to realize the space $\mathscr{F}$ as a subspace of another space $\mathscr{H}$ for which we have a concrete description. For that, we will need the following lemmata.

Lemma 2.3.36 ((COIFMAN et al., 1982), Proposition 2.1). For every $x \in X, x \neq 0$, and every $z_{0} \in \mathbb{D}$ we have

$$
\int_{0}^{2 \pi}\left|\log \|x\|_{z}\right| P_{z_{0}}\left(e^{i t}\right) d t<\infty
$$

Proof. From I2, we have that for every $z \in \mathbb{T}$

$$
\begin{aligned}
\log \|x\|_{\mathscr{U}} & \leq \log \left(k(z)\|x\|_{z}\right) \\
& =\log k(z)+\log \|x\|_{z} \\
& \leq \log ^{+} k(z)+\log \|x\|_{z}
\end{aligned}
$$


so that for every $t \in[0,2 \pi]$

$$
\log \|x\|_{e^{i t}} \geq \log \|x\|_{\mathscr{U}}-\log ^{+} k(z)
$$

and

$$
\int_{0}^{2 \pi} \log \|x\|_{e^{i t}} P_{z_{0}}\left(e^{i t}\right) d t \geq 2 \pi \log \|x\|_{\mathscr{U}}-\int_{0}^{2 \pi} \log ^{+} k\left(e^{i t}\right) P_{z_{0}}\left(e^{i t}\right) d t>-\infty
$$

But

$$
\int_{0}^{2 \pi} \log \|x\|_{e^{i t}} P_{z_{0}}\left(e^{i t}\right) d t=\int_{0}^{2 \pi} \log ^{+}\|x\|_{e^{i t}} P_{z_{0}}\left(e^{i t}\right) d t+\int_{A} \log \|x\|_{e^{i t}} P_{z_{0}}\left(e^{i t}\right) d t
$$

where $A=\left\{t \in[0,2 \pi]:\|x\|_{e^{i t}} \leq 1\right\}$. By I1, we obtain that

$$
\int_{0}^{2 \pi}\left|\log \|x\|_{e^{i t}}\right| P_{z_{0}}\left(e^{i t}\right) d t=\int_{0}^{2 \pi} \log ^{+}\|x\|_{e^{i t}} P_{z_{0}}\left(e^{i t}\right) d t-\int_{A} \log \|x\|_{e^{i t}} P_{z_{0}}\left(e^{i t}\right) d t<\infty
$$

Lemma 2.3.37 ((COIFMAN et al., 1982), Proposition 2.2). If $k$ is a containing function for an interpolation family, then

$$
\int_{\mathbb{T}}|\log k(z)| P_{z_{0}}(z) d z<\infty
$$

Proof. Take $x \in X$ different of 0 . Then by I2

$$
\log \|x\|_{\mathscr{U}} \leq \log k(z)+\log ^{+}\|x\|_{z}
$$

and the proof follows the same reasoning of the proof of Lemma 2.3.36.

The following lemma may be obtained as a consequence of (DUREN, 2000, Theorem 2.9) (Proposition 2.3.48 below). Recall that if $u$ is a harmonic function on $\mathbb{D}$, a harmonic conjugate of $u$ is a harmonic function $\tilde{u}$ such that $u+i \tilde{u}$ is analytic. Harmonic conjugates always exist, and are unique up to a constant.

Lemma 2.3.38 ((SZEGÖ, 1921)). Let $f: \mathbb{T} \rightarrow(0, \infty)$ be a measurable function such that for some (and therefore for all) $z_{0} \in \mathbb{D}$ we have

$$
\int_{0}^{2 \pi}\left|\log f\left(e^{i t}\right)\right| P_{z_{0}}\left(e^{i t}\right) d t<\infty
$$

Let $u$ be given by

$$
u\left(z_{0}\right)=\int_{\mathbb{T}} \log f(z) P_{z_{0}}(z) d z
$$

and let $\tilde{u}$ be a harmonic conjugate of $u$. Then the function $G=e^{u+i \tilde{u}}$ is in $N^{+}$, never vanishes in $\mathbb{D}$, and $|G(z)|=f(z)$ for almost every $z \in \mathbb{T}$. We say that $G$ is an outer function associated to $f$.

Definition 2.3.39. Let $k$ be a containing function for the interpolation family, let $K$ be an outer function associated to $k$, and let $X(z)$ be the closed subspace of $X_{z}$ generated by $X$.

The space $\mathscr{H}$ is the space of all analytic functions $f: \mathbb{D} \rightarrow \mathscr{U}$ such that

H1 $\|f / K\|_{\mathscr{U}}$ is bounded;

$H 2$ The nontangential limit $f(z)$ exists and is in $X(z)$ for almost every $z \in \mathbb{T}$;

$H 3$ The function $z \mapsto\|f(z)\|_{z}$ is essentially bounded on $\mathbb{T}$.

The norm on $\mathscr{H}$ is defined by

$$
\|f\|_{\mathscr{H}}=\underset{z \in \mathbb{T}}{\operatorname{ess} \sup }\|f(z)\|_{z}
$$


Observation 2.3.40. Notice that in the previous definition $\mathrm{H} 3$ implicitly assumes that $z \mapsto$ $\|f(z)\|_{z}$ is measurable. That is the content of the next result.

Proposition 2.3.41. Let $f: \mathbb{D} \rightarrow \mathscr{U}$ be an analytic function satisfying $H 2$. Then the map $z \mapsto$ $\|f(z)\|_{z}$ is measurable.

Proof. Since the domain of $f$ is $\mathbb{D}$ and $f$ is continuous, its image is separable. Also, $f$ is weakly measurable, and therefore, is strongly measurable by Pettis Measurability Theorem ((HYTÖNEN et al., 2016, Chapter 1), for example), i. e., it is the pointwise limit of a sequence of simple measurable functions.

The same remarks apply to the functions $f_{r}(t)=f\left(r e^{i t}\right), 0<r<1, t \in[0,2 \pi)$.

The function $f_{1}(t)=f\left(e^{i t}\right)$ is almost everywhere the pointwise limit of the functions $f_{r}$, and therefore is itself strongly measurable. Therefore, we may approximate $f_{1}$ by simple functions with image in a dense subset of the image of $f_{1}$.

Notice that the closure of $X$ in $\mathscr{U}$ contains $X(z)$ for every $z$. Therefore, we may approximate $f_{1}$ by simple functions with values in $X$, and for such a function, say, $\varphi$, since $x \mapsto\|x\|_{z}$ is measurable for every $z$, it follows that $z \mapsto\|\varphi(z)\|_{z}$ is measurable.

By taking limits, this implies that $z \mapsto\|f(z)\|_{z}$ is measurable.

Corollary 2.3.42. The space $\mathscr{H}$ is a vector space.

Proof. If $f, g \in \mathscr{H}$, then clearly $f+g$ satisfies $\mathrm{H} 1$ and $\mathrm{H} 2$, and therefore satisfies $\mathrm{H} 3$.

Proposition 2.3.43 ((COIFMAN et al., 1982), Proposition 2.3). The space $\mathscr{H}$ is complete.

Proof. Let $\left(h_{n}\right) \subset \mathscr{H}$ be a Cauchy sequence. By H2, we may take a subset of null measure $\mathbb{T}_{0} \subset \mathbb{T}$ such that for every $n$ and every $z \in \mathbb{T} \backslash \mathbb{T}_{0}$ the nontangential limit $h_{n}(z)$ exists and is in $X(z)$.

Furthermore, since $\left(h_{n}\right)$ is Cauchy, for every $l \in \mathbb{N}$ there is $N=N(l) \in \mathbb{N}$ such that if $n, m \geq N$ then

$$
\underset{z \in \mathbb{T}}{\operatorname{ess} \sup }\left\|h_{n}(z)-h_{m}(z)\right\|_{z}<\frac{1}{l}
$$

So we may suppose that for every $z \in \mathbb{T} \backslash \mathbb{T}_{0}$ and every $n, m \geq N(l)$ we have

$$
\left\|h_{n}(z)-h_{m}(z)\right\|_{z} \leq \frac{1}{l}
$$

Therefore, $\left(h_{n}(z)\right)$ is a Cauchy sequence in $X(z)$, and converges to an element $h(z)$ in $X(z)$ in $\|\cdot\|_{z} \cdot$

From $\mathrm{I} 2$ and the fact that $|K|=k$ a. e. we get

$$
\underset{z \in \mathbb{T}}{\operatorname{ess} \sup }\left\|\frac{h_{n}(z)}{K(z)}-\frac{h(z)}{K(z)}\right\|_{\mathscr{U}} \leq \frac{1}{l}
$$

for every $n \geq N(l)$.

Let $u^{*} \in \mathscr{U}^{*}$. By H1, the function $u^{*}\left(\frac{h_{n}}{K}\right): \mathbb{D} \rightarrow \mathbb{C}$ is analytic and bounded for every $n \in \mathbb{N}$. By H2, it has a. e. nontangential limits on $\mathbb{T}$, and for a. e. $z \in \mathbb{T}$ this limits agrees with $u^{*}\left(\frac{h_{n}(z)}{K(z)}\right)$.

By the maximum modulus principle, for every $n, m \in \mathbb{N}$ and every $z_{0} \in \mathbb{D}$ we have

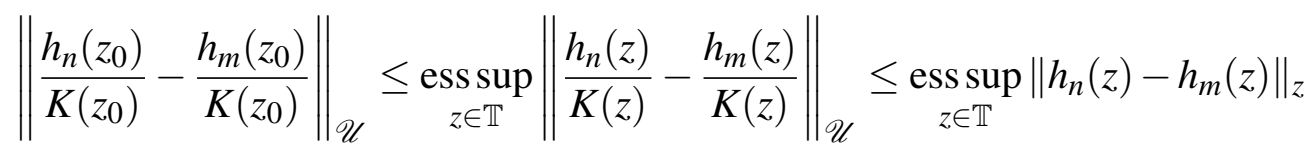


So the functions $\frac{h_{n}}{K}$ converge uniformly on $\mathbb{D}$ to a bounded analytic function $G: \mathbb{D} \rightarrow \mathscr{U}$.

Let $z \in \mathbb{T}$, and let $\left(z_{n}\right)$ be a sequence in $\mathbb{D}$ converging nontangentially to $z$. For each $n$ we may approximate $G\left(z_{n}\right)$ by $\frac{h_{k(n)}\left(z_{n}\right)}{K\left(z_{n}\right)}$ for some $k(n)$, and using better approximations for each $n$ we get that for a. e. $z \in \mathbb{T}$ the nontangential limit $G(z)$ exists and is $\frac{h(z)}{K(z)}$.

Let $h(z)=K(z) G(z)$ for $z \in \mathbb{D}$. Then $h: \mathbb{D} \rightarrow \mathscr{U}$ is an analytic function, $\frac{h}{K}$ is bounded and has a. e. nontangential limits on $\mathbb{T}$ which agree a. e. with $h(z) \in X(z)$ previously defined. Also, $h$ is in $\mathscr{H}$ and $h_{n} \rightarrow h$.

We will need now to use some properties of the Smirnov class.

Definition 2.3.44. A Blaschke product is a function $B$ on $\mathbb{D}$ of the form

$$
B(z)=z^{m} \prod_{n} \frac{\left|a_{n}\right|}{a_{n}} \frac{a_{n}-z}{1-\overline{a_{n}} z}
$$

where $m \geq 0, m \in \mathbb{Z}, a_{n} \in \mathbb{C}$ for every $n$, and $\Sigma\left(1-\left|a_{n}\right|\right)<\infty$.

If the set $\left\{a_{n}\right\}$ is empty, $B(z)=z^{m}$.

Definition 2.3.45. A singular inner function $S$ on $\mathbb{D}$ is a function of the form

$$
S(z)=\exp \left(-\int_{0}^{2 \pi} \frac{e^{i t}+z}{e^{i t}-z} d \mu(t)\right)
$$

where $\mu:[0,2 \pi] \rightarrow \mathbb{R}$ is a bounded nondecreasing function such that $\mu^{\prime}(t)=0$ a.e.

Definition 2.3.46. An outer function for the class $N$ is a function $F$ on $\mathbb{D}$ of the form

$$
F(z)=e^{i \gamma} \exp \left(\frac{1}{2 \pi} \int_{0}^{2 \pi} \frac{e^{i t}+z}{e^{i t}-z} \log \psi(t) d t\right)
$$

where $\gamma \in \mathbb{R}, \psi \geq 0$ and $|\log \psi|$ is integrable.

Lemma 2.3.47. For every $z \in \mathbb{D}$ and every $t \in[0,2 \pi]$ we have

$$
P_{z}\left(e^{i t}\right)+i \tilde{P}_{z}\left(e^{i t}\right)=\frac{e^{i t}+z}{e^{i t}-z}
$$

Proposition 2.3.48. [(DUREN, 2000), Theorem 2.9] A function $\psi: \mathbb{D} \rightarrow \mathbb{C}$ is in the class $N^{+}$if and only if it has a factorization $\psi=B S F$, where $B$ is a Blaschke product, $S$ is a singular inner function, and $F$ is an outer function for the class $N$.

Lemma 2.3.49. Let $\left\{X_{z}: z \in \mathbb{D} ; X\right\}$ be an interpolation family with containing function $k$ and $X \neq 0$. Let $\psi \in N^{+}$and $K$ be an outer function associated to $k$ (Lemma 2.3.38). Then $\frac{\psi}{K} \in N^{+}$. Proof. By Proposition 2.3.48, we have a factorization $\psi=B S F$, where $B$ is a Blaschke product, $S$ is a singular inner function, and $F$ is an outer function for the class $N$. Let $\varphi$ be the function such that

$$
F(z)=e^{i \gamma} \exp \left(\frac{1}{2 \pi} \int_{0}^{2 \pi} \frac{e^{i t}+z}{e^{i t}-z} \log \varphi(t) d t\right)
$$


By Lemma 2.3.47, the function $K$ is given by

$$
K(z)=\exp \left(\frac{1}{2 \pi} \int_{0}^{2 \pi} \frac{e^{i t}+z}{e^{i t}-z} \log k\left(e^{i t}\right) d t\right)
$$

Since $X \neq 0$, we have that $k\left(e^{i t}\right) \neq 0$ for every $t \in[0,2 \pi]$. So, $\frac{\psi}{K}=B S G$, where $G$ is the outer function for the class $N$ given by

$$
G(z)=e^{i \gamma} \exp \left(\frac{1}{2 \pi} \int_{0}^{2 \pi} \frac{e^{i t}+z}{e^{i t}-z} \log \frac{\varphi(t)}{k\left(e^{i t}\right)} d t\right)
$$

This is indeed an outer function for the class $N$ by Lemma 2.3.37. Therefore, by Proposition 2.3.48, we have $\frac{\psi}{K} \in N^{+}$.

Proposition 2.3.50. [(DUREN, 2000), Theorem 2.11] Let $f \in N^{+}$and $p>0$. If $t \mapsto f\left(e^{i t}\right)$ is a function in $L^{p}$, then $f \in H^{p}$.

Proposition 2.3.51 ((COIFMAN et al., 1982), Proposition 2.3). $\mathscr{F}$ is a closed subspace of $\mathscr{H}$. Proof. This follows by noting that $\mathscr{G} \subset \mathscr{H}$ with equality of norms. Indeed, H2 and H3 are clear for functions in $\mathscr{G}$.

For H1, let $g=\sum_{j=1}^{n} \psi_{j} x_{j} \in \mathscr{G}$. We may suppose that the set $\left\{x_{j}\right\}$ is linearly independent. Then the map $\sum a_{j} x_{j} \mapsto \sum a_{j} e_{j}$ defines an isomorphism between $\operatorname{span}\left\{x_{j}: 1 \leq j \leq n\right\} \subset \mathscr{U}$ and $\ell_{\infty}^{n}$. So there is a constant $C>0$ such that for each $1 \leq j \leq n$ we have

$$
\left|\frac{\psi_{j}(z)}{K(z)}\right| \leq C\left\|\frac{g(z)}{K(z)}\right\|_{\mathscr{U}} \leq C\|g\|
$$

for a. e. $z \in \mathbb{T}$.

By Lemma 2.3.49 and Proposition 2.3.50, the function $\frac{\psi_{j}}{K}$ is in $H^{\infty}$ for every $1 \leq j \leq \infty$. Therefore, using (2.3) we get for every $z \in \mathbb{D}$

$$
\left\|\frac{g(z)}{K(z)}\right\|_{\mathscr{U}} \leq \sum\left|\frac{\psi_{j}(z)}{K(z)}\right|\left\|x_{j}\right\|_{\mathscr{U}} \leq C\|g\| \sum\left\|x_{j}\right\|_{\mathscr{U}}
$$

and therefore $\left\|\frac{g}{K}\right\|_{\mathscr{U}}$ is bounded in $\mathbb{D}$.

The following result will be used later in the text.

Lemma 2.3.52. Let $f \in \mathscr{F}$ and $h \in H^{\infty}$. Then $h f \in \mathscr{F}$.

Proof. Notice that this is true if $f \in \mathscr{G}$, since $H^{\infty} \subset N^{+}$, which is closed by multiplication.

Let $\left(g_{n}\right) \subset \mathscr{G}$ be such that $g_{n} \rightarrow f$. Then $h g_{n} \in \mathscr{G}$ for each $n$.

It is clear that $h f \in \mathscr{H}$, and since $h$ is bounded, we have $h g_{n} \rightarrow h f$.

The next proposition is essential to the definition of interpolation spaces.

Proposition 2.3.53. For each $z_{0} \in \mathbb{D}$, the evaluation map $\delta_{z_{0}}: \mathscr{H} \rightarrow \mathscr{U}$ is continuous. 
Proof. Let $u^{*} \in \mathscr{U}^{*}$. Then by $\mathrm{H} 1, u^{*}(f / K)$ is an analytic bounded map from $\mathbb{D}$ into $\mathbb{C}$, and therefore, by I2, we have an estimate:

$$
\left|u^{*}\left(f\left(z_{0}\right) / K\left(z_{0}\right)\right)\right| \leq \underset{z \in \mathbb{T}}{\operatorname{ess} \sup }\left|u^{*}(f(z) / K(z))\right| \leq\left\|u^{*}\right\| \underset{z \in \mathbb{T}}{\operatorname{ess} \sup }\|f(z)\| \mathscr{U} /|K(z)| \leq\left\|u^{*}\right\|\|f\|_{\mathscr{H}}
$$

Taking the supremum over $u^{*}$ with $\left\|u^{*}\right\|=1$, we have that

$$
\left\|f\left(z_{0}\right)\right\|_{\mathscr{U}} \leq\left|K\left(z_{0}\right)\right|\|f\|_{\mathscr{H}}
$$

and therefore $\left\|\delta_{z_{0}}\right\| \leq\left|K\left(z_{0}\right)\right|$.

The following was already noticed in (COIFMAN et al., 1982).

Proposition 2.3.54. Let $x \in X$. Then there is $\psi \in N^{+}$such that $\|\psi(z) x\|_{z}=1$ and $\psi$ never vanishes in $\mathbb{D}$.

Proof. Using Lemmas 2.3.36 and 2.3.38, we may obtain a function $\psi \in N^{+}$such that $\|\psi(z) x\|_{z}=$ 1 for almost every $z \in \mathbb{T}$. Since this $\psi$ is an outer function, it never vanishes.

From now on we omit the subscripts of the norms whenever the context is clear.

The fact that we have the spaces $\mathscr{G}$ and $\mathscr{F}$ allows the definition of two interpolation spaces.

Definition 2.3.55. Let $\left\{X_{z}: z \in \mathbb{T} ; X\right\}$ be an interpolation family, and let $z_{0} \in \mathbb{D}$. Define the space $X_{\left\{z_{0}\right\}}$ to be the completion of $X$ with the norm

$$
\|x\|_{\left\{z_{0}\right\}}=\inf \left\{\|g\|: g \in \mathscr{G}, g\left(z_{0}\right)=x\right\}
$$

(Notice that by Proposition 2.3.54 the set where the infimum is taken in nonempty.)

We also define the space $X_{\left[z_{0}\right]}=\mathscr{F} /\left(\operatorname{ker}\left(\delta_{z_{0}}\right)\right)$, that is,

$$
X_{\left[z_{0}\right]}=\left\{f\left(z_{0}\right): f \in \mathscr{F}\right\}
$$

and

$$
\|x\|_{\left[z_{0}\right]}=\inf \left\{\|f\|: f \in \mathscr{F}, f\left(z_{0}\right)=x\right\}
$$

Observation 2.3.56. The norms $\|\cdot\|_{\left\{z_{0}\right\}}$ and $\|\cdot\|_{\left[z_{0}\right]}$ may be nonequivalent on $X$ (COIFMAN et al., 1982).

Observation 2.3.57. By Proposition 2.3.53, $X_{\left[z_{0}\right]}$ is a Banach space, and by the definition of $\mathscr{F}$, $X \subset X_{\left[z_{0}\right]}$ is dense in $X_{\left[z_{0}\right]}$.

The first objective of interpolation theory is to obtain boundedness of linear maps in the interpolated spaces. This is the object of the following theorem, which is an analogue of Theorem 2.3.10 in the context of families, and is an adaptation of Theorem 4.1 of (COIFMAN et al., 1982) to our definitions.

Theorem 2.3.58. Let $\mathscr{X}=\left\{X_{z}: z \in \mathbb{T} ; X\right\}$ and $\mathscr{Y}=\left\{Y_{z}: z \in \mathbb{T} ; Y\right\}$ be interpolation families with containing spaces $\mathscr{U}$ and $\mathscr{V}$, respectively. Let $M: \mathbb{T} \rightarrow[0, \infty]$ be a function such that $\log M$ is absolutely integrable, and let

$$
N\left(z_{0}\right)=\exp \left(\int_{\mathbb{T}} \log M(z) P_{z_{0}}(z) d z\right)
$$

for every $z_{0} \in \mathbb{D}$. 
1. Let $T: X \rightarrow Y$ be a linear map such that for every $x \in X$ and $a$. $e . z \in \mathbb{T}$ we have $\|T x\|_{B_{z}} \leq M(z)\|x\|_{A_{z}}$. Then for every $z_{0} \in \mathbb{D}$ the map $T$ has a unique extension from $X_{\left\{z_{0}\right\}}$ into $Y_{\left\{z_{0}\right\}}$ with norm at most $N\left(z_{0}\right)$.

2. Let $T: \mathscr{U} \rightarrow \mathscr{V}$ be an operator mapping $X$ into $Y$ such that for every $x \in X$ and a. e. $z \in \mathbb{T}$ we have $\|T x\|_{B_{z}} \leq M(z)\|x\|_{A_{z}}$. Then for every $z_{0} \in \mathbb{D}$ the map $T$ has a unique extension from $X_{\left[z_{0}\right]}$ into $Y_{\left[z_{0}\right]}$ with norm at most $N\left(z_{0}\right)$.

Proof. (1) Using Lemma 2.3.38 we obtain an outer function $G$ such that $|G(z)| M(z)=1$ for a. e. $z \in \mathbb{N}$.

Let $x \in X$ and $g=\sum \psi_{j} x_{j} \in \mathscr{G}(\mathscr{X})$ be such that $g\left(z_{0}\right)=x$. Consider the function $h=$ $G T\left(\sum \psi_{j} x_{j}\right)$. Then for a. e. $z \in \mathbb{T}$ we have

$$
\|h(z)\|_{Y_{z}} \leq|G(z)| M(z)\left\|\sum \psi_{j} x_{j}\right\|_{X_{z}}=\|g(z)\|_{X_{z}} \leq\|g\|
$$

Using the fact that $T: X \rightarrow Y$ and Proposition 2.3.48 we obtain that $h \in \mathscr{G}(\mathscr{Y})$.

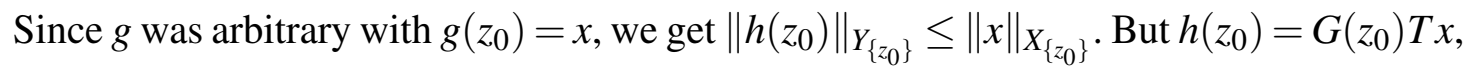
so that

$$
\|T x\|_{Y_{\left\{z_{0}\right\}}} \leq \frac{1}{\left|G\left(z_{0}\right)\right|}\|x\|_{X_{\left\{z_{0}\right\}}}
$$

But $\frac{1}{\left|G\left(z_{0}\right)\right|}=N\left(z_{0}\right)$. Since $X$ is dense in $X_{\left\{z_{0}\right\}}$, we get the result.

(2) Let $x \in X$ and $f \in \mathscr{F}(\mathscr{X})$ be such that $f\left(z_{0}\right)=x$. Take a sequence $g_{n} \in \mathscr{G}(\mathscr{X})$ such that $g_{n} \rightarrow f$.

Let $G$ be as in (1), and let $h_{n}(z)=G T\left(g_{n}(z)\right)$. It follows from the calculations in (1) that $\left(h_{n}\right)$ is a Cauchy sequence in $\mathscr{G}(\mathscr{Y})$. Let $h=\lim h_{n}$.

Now, we have for each $z \in \mathbb{D}$ that $\left\|f(z)-g_{n}(z)\right\|_{\mathscr{U}} \rightarrow 0$, by Proposition 2.3.53. Since $T$ is an operator, it follows that $\left\|G T(f(z))-G T\left(g_{n}(z)\right)\right\|_{\mathscr{V}} \rightarrow 0$, and therefore, by unicity of limits, we have $h(z)=G T(f(z))$ for every $z \in \mathbb{D}$.

Then, by the continuity of $T$, we have that $h(z)=G T(f(z))$ for a. e. $z \in \mathbb{T}$. Therefore:

$$
\begin{aligned}
\|T(x)\|_{\left[z_{0}\right]} & \leq \frac{1}{\left|G\left(z_{0}\right)\right|}\|h\| \\
& =N\left(z_{0}\right) \lim \left\|h_{n}\right\| \\
& \leq N\left(z_{0}\right) \lim \left\|g_{n}\right\| \\
& =N\left(z_{0}\right)\|f\|
\end{aligned}
$$

Since $f$ was arbitrary, we get the result.

The next result gives a standard example of interpolation family.

Theorem 2.3.59 ((COIFMAN et al., 1982), Theorem 5.1). Let $\alpha: \mathbb{T} \rightarrow[0,1]$ be a measurable function, $\left(X_{0}, X_{1}\right)$ be a compatible couple, and for $z \in \mathbb{T}$ let $A_{z}=\left(X_{0}, X_{1}\right)_{\alpha(z)}$.

1. The family $\left\{A_{z}: z \in \mathbb{T} ; X\right\}$ is an interpolation family with containing space $\Sigma\left(X_{0}, X_{1}\right)$, containing function $k \equiv 1$, and $\Delta$ space $X=\cap_{z \in \mathbb{T}}\left(X_{0}, X_{1}\right)_{\alpha(z)}$.

2. For $z_{0} \in \mathbb{D}$, let

$$
\alpha\left(z_{0}\right)=\int_{0}^{2 \pi} \alpha(z) P_{z_{0}}(z) d z
$$

Then $A_{\left[z_{0}\right]}=\left(X_{0}, X_{1}\right)_{\alpha\left(z_{0}\right)}$.

3. If both $\inf _{z \in \mathbb{T}} \alpha(z)$ and $\sup _{z \in \mathbb{T}} \alpha(z)$ are attained, then $A_{\left[z_{0}\right]}=A_{\left\{z_{0}\right\}}$ for every $z_{0} \in \mathbb{D}$. 
We are interested in twisted sums of Banach spaces induced by complex interpolation. As in the case with interpolation of couples, we use the derivative of the analytic functions we work with, and for that we need the boundedness of the evaluation of the derivative. In turn, to prove this boundedness in the case of couples we use the fact that we may divide analytic functions $f$ of the interpolation scale such that $f(\theta)=0$ by conformal maps $\varphi: \mathbb{S} \rightarrow \mathbb{D}$ such that $\varphi(\theta)=0$. As we shall see, in the case of interpolation of families we have this ability to divide by conformal maps if and only if $X_{\left[z_{0}\right]}=X_{\left\{z_{0}\right\}}$.

Lemma 2.3.60. Let $t \geq 0, s>0$. Then

$$
\log ^{+}(t+s) \leq \log (1+t+s) \leq \log (2+t)+\log ^{+} s
$$

Proof. If $\log ^{+}(t+s)=\log (t+s)$, then it is clear that

$$
\log ^{+}(t+s) \leq \log (1+t+s)
$$

If $\log ^{+}(t+s)=0$, then the inequality is also clear, since $1+t+s \geq 1$.

If $s<1$, then $1+t+s \leq 2$, and

$$
\log (1+t+s) \leq \log (2+t)=\log (2+t)+\log ^{+} s
$$

If $s \geq 1$, then $1+t+s \leq s(2+t)$, and therefore

$$
\log (1+t+s) \leq \log (2+t)+\log s=\log (2+t)+\log ^{+} s
$$

Lemma 2.3.61. Let $z_{0} \in \mathbb{D}$ and $f \in N^{+}$be such that $f\left(z_{0}\right)=0$. Define $g: \mathbb{D} \rightarrow \mathbb{C}$ by $g(z)=\frac{f(z)}{z-z_{0}}$, for $z \neq z_{0}$, and $g\left(z_{0}\right)=f^{\prime}\left(z_{0}\right)$. Then $g \in N^{+}$.

Proof. First, we check that $g \in N$. We must prove that the integrals

$$
\int_{0}^{2 \pi} \log \left(1+\left|g\left(r e^{i t}\right)\right|\right) d t
$$

are uniformly bounded in $0<r<1$. Take $s$ such that $\left|z_{0}\right|<s<1$. Since $\{z \in \mathbb{C}:|z| \leq s\}$ is compact, $g$ is bounded in this set, and therefore the integrals (2.4) are uniformly bounded for $0<r \leq s$.

Let $a=s-\left|z_{0}\right|$. Using Lemma 2.3.60, for $s<r<1$ we have

$$
\begin{aligned}
\int_{0}^{2 \pi} \log \left(1+\left|g\left(r e^{i t}\right)\right|\right) d t & =\int_{0}^{2 \pi} \log \left(1+\left|\frac{f\left(r e^{i t}\right)}{r e^{i t}-z_{0}}\right|\right) d t \\
& \leq \int_{0}^{2 \pi} \log \left(1+\left|\frac{f\left(r e^{i t}\right)}{a}\right|\right) d t \\
& =\int_{0}^{2 \pi} \log \left(a+\left|f\left(e^{i t}\right)\right|\right)-\log a d t \\
& \leq \int_{0}^{2 \pi} \log (1+a)+\log ^{+}\left|f\left(r e^{i t}\right)\right|-\log a d t
\end{aligned}
$$

and since $f \in N$, we have that the integrals (2.4) are uniformly bounded in $0<r<1$.

Now, to check that $g \in N^{+}$, for $0<r<1$ and $0<s$ let

$$
A_{r, s}=\left\{t \in[0,2 \pi]:\left|f\left(r e^{i t}\right)-f\left(z_{0}\right)\right|>s\right\}
$$


By using a conformal map, we may suppose that $z_{0}=0$. Then, if we denote by $\Lambda$ the Lebesgue measure, we have

$$
\begin{aligned}
\int_{0}^{2 \pi} \log ^{+}\left|g\left(r e^{i t}\right)\right| d t= & \int_{0}^{2 \pi} \log +\frac{f\left(r e^{i t}\right)-f(0)}{r e^{i t}} \mid d t \\
= & \int_{A_{r, r}} \log \frac{\left|f\left(r e^{i t}\right)-f(0)\right|}{r} d t \\
= & \int_{A_{r, r}} \log \left|f\left(r e^{i t}\right)-f(0)\right| d t-\Lambda\left(A_{r, r}\right) \log r \\
= & \int_{A_{r, 1}} \log \left|f\left(r e^{i t}\right)-f(0)\right| d t \\
& +\int_{A_{r, r} \backslash A_{r, 1}} \log \left|f\left(r e^{i t}\right)-f(0)\right| d t \\
& -\Lambda\left(A_{r, r}\right) \log r \\
= & \int_{0}^{2 \pi} \log { }^{+}\left|f\left(r e^{i t}\right)-f(0)\right| d t \\
& +\int_{A_{r, r} \backslash A_{r, 1}} \log \left|f\left(r e^{i t}\right)-f(0)\right| d t \\
& -\Lambda\left(A_{r, r}\right) \log r
\end{aligned}
$$

But we have

$$
2 \pi \log r \leq \Lambda\left(A_{r, r} \backslash A_{r, 1}\right) \log r \leq \int_{A_{r, r} \backslash A_{r, 1}} \log \left|f\left(r e^{i t}\right)-f(0)\right| d t \leq 0
$$

and

$$
\left|g\left(e^{i t}\right)\right|=\left|f\left(e^{i t}\right)-f(0)\right|
$$

Since $f \in N^{+}$, we obtain

$$
\lim _{r \rightarrow 1^{-}} \int_{0}^{2 \pi} \log ^{+}\left|g\left(r e^{i t}\right)\right| d t=\int_{0}^{2 \pi} \log ^{+}\left|g\left(e^{i t}\right)\right| d t
$$

Proposition 2.3.62. Let $\left\{X_{z}: z \in \mathbb{T} ; X\right\}$ be an interpolation family and $z_{0} \in \mathbb{D}$. Let $\varphi: \mathbb{D} \rightarrow \mathbb{D}$ be a conformal map such that $\varphi\left(z_{0}\right)=0$. Then $X_{\left\{z_{0}\right\}}=X_{\left[z_{0}\right]}$ if and only if for every $f \in \mathscr{F}$ such that $f\left(z_{0}\right)=0$ the function $h$ is in $\mathscr{F}$, where $h: \mathbb{D} \rightarrow \mathscr{U}$ is given by $h(z)=\frac{f(z)}{\varphi(z)}$ for $z \neq z_{0}$ and $h\left(z_{0}\right)=\frac{f^{\prime}\left(z_{0}\right)}{\varphi^{\prime}\left(z_{0}\right)}$

Proof. $(\Rightarrow)$ Suppose $X_{\left\{z_{0}\right\}}=X_{\left[z_{0}\right]}$, and let $f \in \mathscr{F}$ be such that $f\left(z_{0}\right)=0$.

First, we note that $\frac{f}{\varphi} \in \mathscr{H}$. Indeed, $\frac{f}{\varphi K}$ is continuous on $\mathbb{D}$, and therefore is bounded on $|z| \leq \frac{1}{2}$. Since $\varphi$ is open, there is an $r>0$ such that if $\frac{1}{2} \leq z \leq 1$ we have $|\varphi(z)| \geq r$. This implies that for $\frac{1}{2} \leq z \leq 1$ we have

$$
\left\|\frac{f(z)}{\varphi(z) K(z)}\right\|_{\mathscr{U}} \leq \frac{1}{r}\left\|\frac{f(z)}{K(z)}\right\|_{\mathscr{U}}
$$

and therefore we have $\mathrm{H} 1$. 
Also, $\varphi$ has a continuous extension from $\overline{\mathbb{D}}$ onto itself, and therefore $\mathrm{H} 2$ holds. Furthermore, this extension sends $\mathbb{T}$ onto itself, so we have $\mathrm{H} 3$, and $\frac{f}{\varphi} \in \mathscr{H}$.

Let $\varepsilon>0$, and take $g \in \mathscr{G}$ such that $\|f-g\|<\frac{\varepsilon}{2}$. We obtain

$$
\left\|g\left(z_{0}\right)\right\|_{\left\{z_{0}\right\}}=\left\|g\left(z_{0}\right)\right\|_{\left[z_{0}\right]} \leq\|g-f\|<\frac{\varepsilon}{2}
$$

and therefore there is $h \in \mathscr{G}$ such that $h\left(z_{0}\right)=g\left(z_{0}\right)$ and $\|h\|<\frac{\varepsilon}{2}$. Then, we have $g-h \in \mathscr{G}$, $(g-h)\left(z_{0}\right)=0$ and $\|f-(g-h)\| \leq \varepsilon$. This means that we can approximate $f$ by functions in $\mathscr{G}$ in the kernel of $\delta_{z_{0}}$.

Let $g \in \mathscr{G}$ be such that $g\left(z_{0}\right)=0$. We may write $g=\sum \psi_{j} x_{j}$, with $x_{j} \in X$ and $\psi_{j} \in N^{+}$. We may suppose that the $x_{j}$ are linearly independent, and then we have $\psi_{j}\left(z_{0}\right)=0$ for every $j$.

If $\psi \in N^{+}$is such that $\psi\left(z_{0}\right)=0$, then $\zeta=\frac{\psi}{\varphi} \in N^{+}$. Indeed, we have $\zeta \circ \varphi^{-1}=\frac{\psi \circ \varphi^{-1}}{z}$, and since $\psi \circ \varphi^{-1} \in N^{+}$(the classes $N$ and $N^{+}$are invariant by composition with conformal maps), we obtain by Lemma 2.3.61 that $\zeta \circ \varphi^{-1} \in N^{+}$, and therefore $\zeta \in N^{+}$.

This implies that $\frac{g}{\varphi} \in \mathscr{G}$.

Let $\left(g_{n}\right)$ be a sequence in $\mathscr{G}$ such that $g_{n}\left(z_{0}\right)=0$ for every $n$ and $g_{n} \rightarrow f$. Then $\frac{g_{n}}{\varphi} \in \mathscr{G}$ and converges to $\frac{f}{\varphi} \in \mathscr{H}$, and therefore $\frac{f}{\varphi} \in \mathscr{F}$.

$(\Leftarrow)$ Let us prove that $\mathscr{G} \cap \operatorname{ker}\left(\delta_{z_{0}}\right)$ is dense in $\operatorname{ker}\left(\delta_{z_{0}}\right)$. We use an argument of Stafney ((STAFNEY, 1969), Lemma 2.5).

Let $f \in \operatorname{ker}\left(\delta_{z_{0}}\right)$ and $\varepsilon>0$. Take $g \in \mathscr{G}$ such that $\left\|\frac{f}{\varphi}-g\right\|<\varepsilon$. We have that $\varphi g \in$ $\mathscr{G} \cap \operatorname{ker}\left(\delta_{z_{0}}\right)$, and obtain $\|f-\varphi g\|<\varepsilon$. Since $f$ and $\varepsilon$ were arbitrary, $\mathscr{G} \cap \operatorname{ker}\left(\delta_{z_{0}}\right)$ is dense in $\operatorname{ker}\left(\delta_{z_{0}}\right)$. We have

Now, let $x \in X$, and take $f \in \mathscr{G}$ such that $f\left(z_{0}\right)=x$ (which exists by Proposition 2.3.54).

$$
\|x\|_{\left[z_{0}\right]}=\inf \left\{\|f-h\|: h \in \operatorname{ker}\left(\delta_{z_{0}}\right)\right\} \leq\|x\|_{\left\{z_{0}\right\}}
$$

Let $\varepsilon>0$. Given $h \in \operatorname{ker}\left(\delta_{z_{0}}\right)$, take $g \in \mathscr{G} \cap \operatorname{ker}\left(\delta_{z_{0}}\right)$ such that $\|h-g\|<\varepsilon$. Then $h-g \in \operatorname{ker}\left(\delta_{z_{0}}\right)$ and $\|f-g\| \leq\|f-h\|+\varepsilon$. Since $\varepsilon$ and $h$ are arbitrary, this implies that

$$
\|x\|_{\left\{z_{0}\right\}}=\inf \left\{\|f-g\|: g \in \mathscr{G} \cap \operatorname{ker}\left(\delta_{z_{0}}\right)\right\} \leq\|x\|_{z_{0}}
$$

But $X$ is dense in $X_{\left\{z_{0}\right\}}$ and in $X_{\left[z_{0}\right]}$, and therefore $X_{\left\{z_{0}\right\}}=X_{\left[z_{0}\right]}$.

Proposition 2.3.63. Let $\left\{X_{z}: z \in \mathbb{T} ; X\right\}$ be an interpolation family and let $z_{0} \in \mathbb{D}$. Suppose that $X_{\left\{z_{0}\right\}}=X_{\left[z_{0}\right]}$. Then $\delta_{z_{0}}^{\prime}: \operatorname{ker}\left(\delta_{z_{0}}\right) \rightarrow X_{\left[z_{0}\right]}$ is bounded and onto.

Proof. Let $\varphi: \mathbb{D} \rightarrow \mathbb{D}$ be a conformal map such that $\varphi\left(z_{0}\right)=0$.

Let $f \in \operatorname{ker}\left(\delta_{z_{0}}\right)$. Then by Proposition 2.3.62 we have that $h=\frac{f}{\varphi} \in \mathscr{F}$, and $h\left(z_{0}\right)=\frac{f^{\prime}\left(z_{0}\right)}{\varphi^{\prime}\left(z_{0}\right)}$. But $\|h\|=\|f\|$, and therefore

$$
\left\|f^{\prime}\left(z_{0}\right)\right\|_{\left[z_{0}\right]} \leq\left|\varphi^{\prime}\left(z_{0}\right)\right|\|h\|=\left|\varphi^{\prime}\left(z_{0}\right)\right|\|f\|
$$

so that $\left\|\delta_{z_{0}}^{\prime}: \operatorname{ker}\left(\delta_{z_{0}}\right) \rightarrow X_{\left[z_{0}\right]}\right\| \leq\left|\varphi^{\prime}\left(z_{0}\right)\right|$.

To see that the map is onto, take $x \in X_{\left[z_{0}\right]}$ and $f \in \mathscr{F}$ such that $f\left(z_{0}\right)=x$. Then $g=\varphi f \in$ $\operatorname{ker}\left(\delta_{z_{0}}\right)$, and $g^{\prime}\left(z_{0}\right)=\varphi^{\prime}\left(z_{0}\right) x$.

Definition 2.3.64. Let $\left\{X_{z}: z \in \mathbb{T} ; X\right\}$ be an interpolation family and let $z_{0} \in \mathbb{D}$. The family will be called admissible at $z_{0}$ if $X_{\left\{z_{0}\right\}}=X_{\left[z_{0}\right]}$. In this case, we will write $X_{z_{0}}=X_{\left\{z_{0}\right\}}=X_{\left[z_{0}\right]}$. If the family is admissible at every $z_{0} \in \mathbb{D}$, then we will say that the family is admissible. 
Observation 2.3.65. The family $\left\{X_{z}: z \in \mathbb{T} ; X\right\}$ being admissible is equivalent to $\mathscr{F}$ being admissible in the sense of (KALTON; MONTGOMERY-SMITH, 2003), which in turn implies that the family $\left\{X_{z}: z \in \mathbb{D}\right\}$ if an analytic family of Banach spaces using the terminology of the aforementioned work.

Now, we see that if we have an admissible family at $z_{0}$, then we have, as in the case of interpolation of couples, a diagram

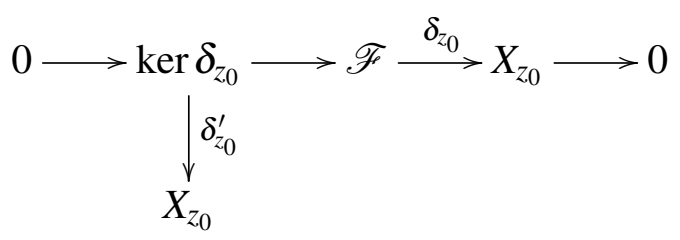

which by Proposition 2.3.13 may be completed into a diagram

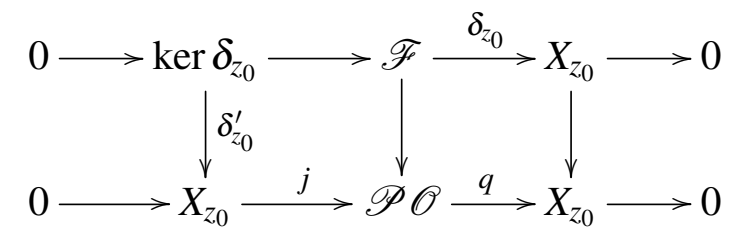

Again, this twisted sum also has the following alternative descriptions:

Proposition 2.3.66 ((KALTON; MONTGOMERY-SMITH, 2003)). Each of the following twisted sums is equivalent to $\mathscr{P} \mathscr{O}$ :

1.

$$
0 \longrightarrow X_{z_{0}} \longrightarrow \mathscr{F} /\left(\operatorname{ker}\left(\delta_{z_{0}}\right) \cap \operatorname{ker}\left(\delta_{z_{0}}^{\prime}\right)\right) \longrightarrow X_{z_{0}} \longrightarrow 0
$$

where we may write

$$
\mathscr{F} /\left(\operatorname{ker}\left(\delta_{z_{0}}\right) \cap \operatorname{ker}\left(\delta_{z_{0}}^{\prime}\right)\right)=\left\{\left(f\left(z_{0}\right), f^{\prime}\left(z_{0}\right)\right): f \in \mathscr{F}\right\}
$$

with the quotient norm, the inclusion is $y \mapsto(0, y)$ and the quotient is $(x, y) \mapsto x$.

2.

$$
0 \longrightarrow X_{z_{0}} \longrightarrow d_{\Omega_{z_{0}}} X_{z_{0}} \longrightarrow X_{z_{0}} \longrightarrow 0
$$

where, if $B_{z_{0}}$ is a bounded section for $\delta_{z_{0}}, \Omega_{z_{0}}=\delta_{z_{0}}^{\prime} B_{z_{0}}: X_{z_{0}} \rightarrow \mathscr{U}$,

$$
d_{\Omega_{z_{0}}} X_{z_{0}}=\left\{(x, y) \in \mathscr{U} \times X_{z_{0}}: x-\Omega_{z_{0}} y \in X_{z_{0}}\right\}
$$

with the quasinorm $\|(x, y)\|=\left\|x-\Omega_{z_{0}} y\right\|_{z_{0}}+\|y\|_{z_{0}}$, the inclusion is $x \mapsto(x, 0)$ and the quotient map is $(x, y) \mapsto y$.

As in Definition 2.3.15, we will say that $\Omega_{z_{0}}$ is trivial when the natural copy of $X_{z_{0}}$ is complemented in $d_{\Omega_{z_{0}}} X_{z_{0}}$.

We now show that the derived space obtained by complex interpolation of a family consisting of only two spaces on the left and right side of the strip is the same as the derived space obtained from complex interpolation of couples.

Theorem 2.3.67. Let $\left(X_{0}, X_{1}\right)$ be a compatible couple of Banach spaces, and let $\delta_{0}, \delta_{1} \subset \mathbb{T}$ be two disjoint arches such that $\mathbb{T}=\partial_{0} \cup \partial_{1}$. Consider the interpolation family $\left\{A_{z}: z \in \mathbb{T} ; X_{0} \cap X_{1}\right\}$, where $A_{z}=X_{j}$ on $\partial_{j}, j=0,1$.

Let $\varphi: \mathbb{D} \rightarrow \mathbb{S}$ be a conformal map such that $\varphi(j+i t) \in \partial_{j}, j=0,1, t \in \mathbb{R}$. Then, for every $z_{0} \in \mathbb{D}$, we have that $d A_{z_{0}}$ is projectively equivalent to $d X_{\varphi\left(z_{0}\right)}$. 
Proof. From Theorem 2.3.59, we already know that $A_{z_{0}} \equiv X_{\varphi\left(z_{0}\right)}$.

We must find a commutative diagram

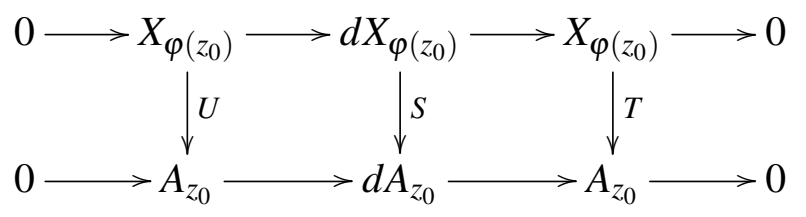

where the vertical arrows are isomorphisms. We will consider $d A_{z_{0}}$ and $d X_{\varphi\left(z_{0}\right)}$ as quotients of the spaces of analytic functions of interpolation.

Let $U=I d, S(x, y)=\left(x, \varphi^{\prime}\left(z_{0}\right) y\right)$, and $T=\varphi^{\prime}\left(z_{0}\right) I d$. It is clear then that $U$ and $T$ are isomorphisms and that the diagram is commutative.

To see that $S$ is bounded, we show that it is bounded on $\Delta(\bar{X}) \times \Delta(\bar{X})$, which is dense in $d X_{\varphi\left(z_{0}\right)}$ by the fact that $\mathscr{G}$ is dense in $\mathscr{F}$.

Given $(x, y) \in \Delta(\bar{X}) \times \Delta(\bar{X})$ take a function $f \in \mathscr{F}_{0}(\bar{X})$ such that $f\left(\varphi\left(z_{0}\right)\right)=x$ and $f^{\prime}\left(\varphi\left(z_{0}\right)\right)=y$. Consider the function $g: \mathbb{D} \rightarrow \sum(\bar{X})$ given by $g=f \circ \varphi$. Then $g\left(z_{0}\right)=x, g^{\prime}\left(z_{0}\right)=$ $\varphi^{\prime}\left(z_{0}\right) y$, and $\|g\|=\|f\|$.

This implies that $\|S(x, y)\| \leq\|(x, y)\|$. Therefore, $S$ is bounded, and by the 3-Lemma 2.2.17 is an isomorphism.

The following result says that we may interpolate using the integral norm on $\mathscr{F}$ and the resulting space is the same.

Proposition 2.3.68 ((COIFMAN et al., 1982), Proposition 2.4). Let $\left\{X_{z}: z \in \mathbb{T} ; X\right\}$ be an interpolation family admissible at $z_{0} \in \mathbb{D}$. Then, for every $x \in X_{z_{0}}$, we have

$$
\|x\|_{z_{0}}=\inf \left\{\exp \int_{\mathbb{T}} \log \|f(z)\|_{z} P_{z_{0}}(z) d z\right\}=\inf \left\{\int_{\mathbb{T}}\|f(z)\|_{z} P_{z_{0}}(z) d z\right\}
$$

where the infimum is taken over all $f \in \mathscr{F}$ such that $f\left(z_{0}\right)=x$.

Proof. Let $f$ be as in the set where the infimum is being taken.

Since $t \mapsto\left\|f\left(e^{i t}\right)\right\|_{X_{e} i t}$ is essentially bounded, for each $n$ the function $v_{n}$ given by

$$
e^{i t} \mapsto \max \left\{\frac{1}{n},\left\|f\left(e^{i t}\right)\right\|_{X_{e^{i t}}}\right\}
$$

is log-integrable, so that we may use Lemma 2.3.38 to obtain outer functions $u_{n}$ such that

$$
\left|u_{n}(z)\right| v_{n}(z)=1
$$

for a. e. $z \in \mathbb{T}$. Since $u_{n}$ is an outer function and on $\mathbb{T}$ it is essentially bounded by $n$, we obtain that $u_{n} f \in \mathscr{F}$. Also

$$
\left\|u_{n}\left(z_{o}\right) f\left(z_{0}\right)\right\|_{X_{z_{0}}} \leq\left\|u_{n} f\right\| \leq 1
$$

so that

$$
\left\|f\left(z_{0}\right)\right\|_{X_{z_{0}}} \leq \frac{1}{\left|u_{n}\left(z_{0}\right)\right|}=\exp \int_{\mathbb{T}} \log v_{n}(z) d P_{z_{0}}(z) d z
$$

and it follows from the monotone convergence theorem that

$$
\left\|f\left(z_{0}\right)\right\|_{X_{z_{0}}} \leq \int_{\mathbb{T}} \log \|f(z)\|_{z} d P_{z_{0}}(z)
$$


so we obtain the first inequality. For the second, apply Jensen's inequality to the first. To obtain the equalities, it is enough to notice that

$$
\int_{\mathbb{T}}\|f(z)\|_{z} P_{z_{0}}(z) d z \leq\|f\|
$$

Recall that complex interpolation of couples was defined on the strip, not on the disk. We show how complex interpolation of families may be defined on the strip. The method of (COIFMAN et al., 1982) is defined for bounded domains $G \subset \mathbb{C}$ conformally equivalent to the disk.

Let us suppose from now on that $G \subset \mathbb{C}$ is such that there is a conformal map $\varphi: \mathbb{D} \rightarrow G$ such that $\varphi$ extends continuously a. e. from $\mathbb{T}$ into $\partial G$ (by Caratheodory's Theorem, this is always the case if the boundary of $G$ is a Jordan curve, but it is also true for other domains, for example, $G=\mathbb{S})$. Let $z_{0} \in G$ be such that $\varphi(0)=z_{0}$.

A family of Banach spaces $\mathscr{X}=\left\{X_{z}: z \in \partial G ; X\right\}$ will be called an interpolation family if the corresponding family $\mathscr{Y}=\left\{Y_{z}=X_{\varphi(z)}: z \in \mathbb{T} ; Y=X\right\}$ is an interpolation family (we may repeat one space on the points where $\varphi$ is not defined), and we define the corresponding interpolation spaces $X_{\left[z_{0}\right]}$ and $X_{\left\{z_{0}\right\}}$ as $Y_{[0]}$ and $Y_{\{0\}}$, respectively. Likewise, the family is called admissible at $z_{0}$ if $X_{\left[z_{0}\right]}=X_{\left\{z_{0}\right\}}$, and is called admissible if it is admissible at every $z_{0} \in \mathbb{S}$.

Notice that there is ambiguity in the choice of the conformal map $\varphi$, but since any conformal map of the disk onto itself which maps 0 to 0 must be a rotation, there is no ambiguity in the definition of the interpolation spaces. Indeed, if $\psi$ is another conformal map and $\mathscr{Z}=$ $\left\{Z_{z}=X_{\psi(z)}: z \in \mathbb{D} ; Z=X\right\}$ is the corresponding family, we have that the action from $\mathscr{F}(\mathscr{Y})$ onto $\mathscr{F}(\mathscr{Z})$ given by composition on the right with $\varphi^{-1} \circ \psi$ sends $\operatorname{ker}\left(\delta_{0}\right) \subset \mathscr{F}(\mathscr{Y})$ onto $\operatorname{ker}\left(\delta_{0}\right) \subset \mathscr{F}(\mathscr{Z})$. Since the same is true for $\operatorname{ker}\left(\delta_{0}\right) \cap \operatorname{ker}\left(\delta_{0}^{\prime}\right)$, we may define unambiguously the twisted sum $\mathscr{F} /\left(\operatorname{ker}\left(\delta_{0}\right) \cap \operatorname{ker}\left(\delta_{0}^{\prime}\right)\right)$, and also its equivalent representation $d_{\Omega_{z_{0}}} X_{z_{0}}$.

It will be useful to have estimates like those of Proposition 2.3.68 for the interpolation of families on the strip. We also take the opportunity to unify notation. From now on we always assume that if $\varphi: \mathbb{D} \rightarrow G$ is a conformal map then it extends continuously a. e. to $\mathbb{T}$.

Definition 2.3.69. Let $z_{0} \in \mathbb{D}$. By $\mu_{z_{0}}$ we denote the probability measure on $\mathbb{T}$ induced by $\frac{P_{z_{0}}}{2 \pi}$.

Definition 2.3.70. Let $z_{0} \in \mathbb{S}$, and let $\varphi: \mathbb{D} \rightarrow G$ be a conformal map such that $\varphi(0)=z_{0}$. Let $\mu_{z_{0}}$ be the probability measure defined on $\partial G$ by

$$
\mu_{z_{0}}(A)=\int_{\varphi^{-1}(A)} P_{0}(z) d z
$$

If $G=\mathbb{S}$, we denote by $\mu_{z_{0}}^{j}$ the corresponding probability measures on $\mathbb{S}_{j}, j=0,1$.

Notice that since any conformal automorphism of the disk sending 0 to 0 is a rotation, and rotations preserve measure on $\mathbb{T}$, it follows that the definitions are independent of the choice of the conformal map $\varphi$.

Also, there is ambiguity with the notation $\mu_{z_{0}}$ if $z_{0} \in \mathbb{D} \cap G$. Whenever we use this notation it will be clear from the context if we are considering a measure on $\mathbb{T}$ or on $\partial G$.

Proposition 2.3.71. Let $z_{0} \in \mathbb{S}$. Then $\mu_{z_{0}}\left(\mathbb{S}_{0}\right)=1-\operatorname{Re}\left(z_{0}\right)$ and $\mu_{z_{0}}\left(\mathbb{S}_{1}\right)=\operatorname{Re}\left(z_{0}\right)$. 
Proof. Let $f: \overline{\mathbb{S}} \rightarrow \overline{\mathbb{S}}$ be given by $f(z)=z$, and let $\varphi: \mathbb{D} \rightarrow \mathbb{S}$ be a conformal map such that $\varphi(0)=z_{0}$. Then $\left.\operatorname{Re}(f(z))\right|_{\mathbb{S}_{0}}=0$ and $\left.\operatorname{Re}(f(z))\right|_{\mathbb{S}_{1}}=1$.

By the Poisson kernel representation 2.3.21, we have that:

$$
\begin{aligned}
\operatorname{Re}\left(z_{0}\right) & =\operatorname{Re}\left(f\left(z_{0}\right)\right) \\
& =\operatorname{Re}(f \circ \varphi(0)) \\
& =\int_{\mathbb{T}} \operatorname{Re}(f \circ \varphi(z)) P_{0}(z) d z \\
& =\int_{\varphi^{-1}\left(\mathbb{S}_{1}\right)} P_{0}(z) d z \\
& =\mu_{z_{0}}\left(\mathbb{S}_{1}\right)
\end{aligned}
$$

Since $\mu_{z_{0}}$ is a probability measure, we have the result.

Definition 2.3.72. Let $\left\{X_{z}: z \in \partial G ; X\right\}$ be an interpolation family admissible at $z_{0} \in G$, and let $\varphi: \mathbb{D} \rightarrow G$ be a conformal map such that $\varphi(0)=z_{0}$ and which extends continuously a. e. to $\mathbb{T}$. Let $\mathscr{G}$ and $\mathscr{F}$ be the spaces of analytic functions associated to the family $\left\{X_{\varphi(z)}: z \in \mathbb{T} ; X\right\}$.

We define:

$$
\mathscr{G}_{z_{0}}=\left\{g \circ \varphi^{-1}: g \in \mathscr{G}\right\}
$$

and

$$
\mathscr{F}_{z_{0}}=\left\{f \circ \varphi^{-1}: f \in \mathscr{F}\right\}
$$

with the norms $\left\|g \circ \varphi^{-1}\right\|_{\mathscr{G}_{z_{0}}}=\|g\|_{\mathscr{G}}$ and $\left\|f \circ \varphi^{-1}\right\|_{\mathscr{F}_{z_{0}}}=\|f\|_{\mathscr{F}}$.

By $\delta_{z_{0}}$ and $\delta_{z_{0}}^{\prime}$ we denote the evaluation at $z_{0}$ and the evaluation of the derivative at $z_{0}$, respectively, on $\mathscr{F}_{z_{0}}$.

Notice that with these definitions we have the identities:

$$
X_{z_{0}}=\mathscr{F}_{z_{0}} / \operatorname{ker}\left(\delta_{z_{0}}\right)
$$

and

$$
d X_{z_{0}} \cong \mathscr{F}_{z_{0}} /\left(\operatorname{ker}\left(\delta_{z_{0}}\right) \cap \operatorname{ker}\left(\delta_{z_{0}}^{\prime}\right)\right)
$$

While the definitions of $\mathscr{G}_{z_{0}}$ and $\mathscr{F}_{z_{0}}$ depend on the choice of the conformal map $\varphi$, these identities do not. Therefore, we omit the dependence on $\varphi$ in what follows.

Proposition 2.3.73. Let $\left\{X_{z}: z \in \partial G ; X\right\}$ be an interpolation family admissible at $z_{0} \in \mathbb{D}$, and let $\varphi: \mathbb{D} \rightarrow G$ be a conformal map such that $\varphi(0)=z_{0}$. Then, for every $x \in X_{z_{0}}$, we have

$$
\|x\|_{z_{0}}=\inf \left\{\exp \int_{\partial \mathbb{S}} \log \|f(z)\|_{z} d \mu_{z_{0}}(z)\right\}=\inf \left\{\int_{\partial \mathbb{S}}\|f(z)\|_{z} d \mu_{z_{0}}(z)\right\}
$$

where the infimum is taken over all $f \in \mathscr{F}_{z_{0}}$ such that $f\left(z_{0}\right)=x$.

Proof. This follows from Proposition 2.3.68 and the definitions of $\mu_{z_{0}}, X_{z_{0}}$ and $\mathscr{F}_{z_{0}}$.

By Proposition 2.3.73, the definition of $\mu_{z_{0}}$ implies:

Proposition 2.3.74. Let $\left\{X_{z}: z \in \partial \mathbb{S} ; X\right\}$ be an interpolation family admissible at $z_{0} \in \mathbb{D}$, and let $\varphi: \mathbb{D} \rightarrow \mathbb{S}$ be a conformal map such that $\varphi(0)=z_{0}$. Then, for every $f \in \mathscr{F}$, we have:

$$
\left\|f\left(z_{0}\right)\right\|_{z_{0}} \leq\left(\int_{\mathbb{S}_{0}}\|f(z)\|_{z} d \mu_{z_{0}}^{0}(z)\right)^{1-\operatorname{Re}\left(z_{0}\right)}\left(\int_{\mathbb{S}_{1}}\|f(z)\|_{z} d \mu_{z_{0}}^{1}(z)\right)^{\operatorname{Re}\left(z_{0}\right)}
$$


Proof. Simply apply Jensen's inequality to the first equality of Proposition 2.3.73 and use Proposition 2.3.71.

In what follows we will often enunciate and prove results for interpolation families on both $\mathbb{T}$ and $\partial \mathbb{S}$. In general, we will have fixed some point $z_{0}$ in $\mathbb{D}$ or in $\mathbb{S}$. In the second case, we will often write $\mathscr{G}$ and $\mathscr{F}$ for $\mathscr{G}_{z_{0}}$ and $\mathscr{F}_{z_{0}}$ without further remarks.

\subsection{Ferenczi's spaces}

The aim of this section is to illustrate the reason we chose to modify the interpolation method from (COIFMAN et al., 1982). We do this by showing how Ferenczi's spaces (FERENCZI, 1997) may be obtained from the interpolation method we presented.

Ferenczi's spaces are examples of uniformly convex hereditarily indecomposable Banach spaces, and are obtained through a method of complex interpolation for families which may be used when the spaces have a common bimonotone Schauder basis.

If a function $f: \mathbb{S} \rightarrow c_{00}$ is analytic, then its range has finite support. So, we may consider the space $\mathscr{A}$ of such functions which are in $L_{1}\left(\partial \mathbb{S}, d \mu_{\frac{1}{2}}\right)$ (any point instead of $\frac{1}{2}$ would work) and such that

$$
f\left(z_{0}\right)=\int_{\partial \mathbb{S}} f(z) d \mu_{z_{0}}(z)
$$

Let $n \in \mathbb{N}$ and consider a family of norms $\|\cdot\|_{z}$ on $\mathbb{C}^{n}, z \in \partial \mathbb{S}$, such that

- $z \mapsto\|x\|_{z}, z \in \partial \mathbb{S}$, is measurable for every $x \in \mathbb{C}^{n}$;

- There are log-integrable functions $c, C: \partial \mathbb{S} \rightarrow \mathbb{R}$ such that for every $z \in \partial \mathbb{S}, x \in \mathbb{C}^{n}$ we have

$$
c(z)\|x\|_{2} \leq\|x\|_{z} \leq C(z)\|x\|_{2}
$$

Then the interpolated norm $\|.\|_{\theta}$, for $0<\theta<1$ is given by

$$
\|x\|_{\theta}=\inf \left(\int_{\partial \mathbb{S}}\|f(z)\|_{z}^{r} d \mu(z)\right)^{\frac{1}{r}}
$$

where $r \geq 1$ is a fixed number (any $r$ gives the same definition of $\|\cdot\|_{\theta}$ ), $x \in \mathbb{C}^{n}$, and the infimum is over all functions $f$ in $\mathscr{A}$ such that $f(\theta)=x$ and $f(z) \in \mathbb{C}^{n}$ for every $z \in \mathbb{S}$. Let us denote

$$
\|f\|_{r}=\left(\int_{\partial \mathbb{S}}\|f(z)\|_{z}^{r} d \mu(z)\right)^{\frac{1}{r}}
$$

for $1 \leq r<\infty$ and

$$
\|f\|_{\infty}=\sup _{z \in \partial \mathbb{S}}\|f(z)\|_{z}
$$

Let $\left\{X_{z}: z \in \partial \mathbb{S}\right\}$ be a family of Banach spaces such that $\left(e_{n}\right) \subset c_{00}$ is a bimonotone Schauder basis for $X_{z}$ for every $z \in \partial \mathbb{S}$, and suppose that

- $z \mapsto\|x\|_{z}, z \in \partial \mathbb{S}$, is measurable for every $x \in c_{00}$;

- $\|\cdot\|_{\infty} \leq\|\cdot\|_{z} \leq\|\cdot\|_{1}$ for every $z \in \partial \mathbb{S}$.

Let $\|.\|_{z}^{(n)}$ be the family of norms on $\mathbb{C}^{n}$ induced by $X_{z}$, and let $\|\cdot\|_{\theta}^{(n)}$ be the interpolated norm associated to $\left(\|\cdot\|_{z}^{(n)}\right)$. Let $X_{\theta}^{(n)}=\left(\mathbb{C}^{n},\|\cdot\|_{\theta}^{(n)}\right)$, and

$$
X_{\theta}^{\mathscr{A}}=\overline{\bigcup_{n} X_{\theta}^{(n)}}
$$


Let us show that $X_{\theta}^{\mathscr{A}}$ may be obtained from our complex interpolation method quite naturally. That is, we will show that $X_{\theta}^{\mathscr{A}}$ coincides with $X_{\{\theta\}}=X_{[\theta]}$ for the interpolation family $\left\{X_{z}: z \in \partial \mathbb{S} ; c_{00}\right\}$. Notice that given the arbitrariness of the family we have no hope of calculating the actual intersection space in general.

The containing space is $\ell_{\infty}$, and therefore we may take as containing function $k \equiv 1$. In particular, $K \equiv 1$ is an outer function associated to $k$.

Let $P_{n}: X_{z} \rightarrow \mathbb{C}^{n}$ be the natural projection. Then $\left\|P_{n}\right\|=1$ in each space $X_{z}, z \in \partial \mathbb{S}$. Also, let $\varphi: \mathbb{D} \rightarrow \mathbb{S}$ be a conformal map such that $\varphi(0)=\theta$. We will take $r=\infty$.

Let $x \in \mathbb{C}^{n}$ and $f \in \mathscr{A}$ be such that $f(\theta)=x, f(z) \in \mathbb{C}^{n}$ for every $z \in \mathbb{S}$ and $\|f\|_{\infty}<\infty$. Then we may write

$$
f(z)=\sum_{j=1}^{n} \psi_{j}(z) e_{j}
$$

where $\psi_{j}: \mathbb{S} \rightarrow \mathbb{C}$ are analytic functions, $j=1, \ldots, n$. Since $f$ satisfies the Poisson kernel representation (2.5), we have that $\psi_{j} \circ \varphi \in H^{1} \subset N^{+}$for each $j$.

So, $f \in \mathscr{G}$, and therefore

$$
\|x\|_{[\theta]} \leq\|x\|_{\{\theta\}} \leq\|x\|_{\theta}^{(n)}
$$

Let $f \in \mathscr{F}$ be such that $f(\theta)=x$. Again, we may write

$$
f(z)=\sum_{j=1}^{\infty} \psi_{j}(z) e_{j}
$$

for a family of analytic functions $\psi_{j}, j \in \mathbb{N}$.

Let $g$ be given by

$$
g(z)=\sum_{j=1}^{n} \psi_{j}(z) e_{j}
$$

Then since $\left\|P_{n}\right\|=1$ on $X_{z}$ for every $z$, we have that $g \in \mathscr{F}, g(\theta)=x$ and $\|g\| \leq\|f\|$. Also, since $f$ is bounded as a function with image in $\ell_{\infty}$, we have that so is $g$.

So each function $\psi_{j} \circ \varphi$ is in $H^{\infty} \subset H^{1}$. Since $H^{1}$ is the space of functions which satisfy the Poisson kernel representation, we have that $g \in \mathscr{A}$, and therefore

$$
\|x\|_{\theta} \leq\|x\|_{[\theta]}
$$

Since $c_{00}$ is dense in $X_{\theta}^{\mathscr{A}}, X_{[\theta]}$ and in $X_{\{\theta\}}$, we obtain the equality of the spaces.

In particular, Ferenczi's spaces from (FERENCZI, 1997) may be obtained from our complex interpolation method.

\subsection{Complex interpolation of couples through $L_{p}$-norms}

To finish the chapter, we discuss interpolation of couples of Banach spaces through a variation of the space $\mathscr{F}(\bar{X})$.

Definition 2.5.1. Let $\bar{X}=\left(X_{0}, X_{1}\right)$ be a compatible couple of Banach spaces. Given $\theta \in(0,1)$ and $p \in[1, \infty)$, the space $\mathscr{F}_{p}^{\theta}=\mathscr{F}_{p}^{\theta}(\bar{X})$ is the space of functions $f: \overline{\mathbb{S}} \rightarrow \Sigma(\bar{X})$ such that

1. $f$ is analytic on $\mathbb{S}$; 
2. $\left.f\right|_{\mathbb{S}_{j}} \in L_{p}\left(\mathbb{S}_{j}, \mu_{\theta}^{j}, X_{j}\right), j=0,1$;

3. For every $z_{0} \in \mathbb{S}$ we have

$$
f\left(z_{0}\right)=\int_{\partial \mathbb{S}} f(z) d \mu_{z_{0}}(z)
$$

4. We have

$$
\|f\|_{p}^{p}=(1-\theta) \int_{\mathbb{S}_{0}}\|f(z)\|_{X_{0}}^{p} d \mu_{\theta}^{0}(z)+\theta \int_{\mathbb{S}_{1}}\|f(z)\|_{X_{1}}^{p} d \mu_{\theta}^{1}(z)<\infty
$$

It is a classical fact that $\mathscr{F}_{p}^{\theta}$ is a Banach space. Also, we have isometrically $\mathscr{F}_{p}^{\theta} / \operatorname{ker}\left(\boldsymbol{\delta}_{\theta}\right)=$ $X_{\theta}$ (PISIER, 2016). We also have that $\mathscr{F}_{p}^{\theta} /\left(\operatorname{ker}\left(\delta_{\theta}\right) \cap \operatorname{ker}\left(\delta_{\theta}^{\prime}\right)\right)$ is an extension of $X_{\theta}$ equivalent to $d X_{\theta}$. We present extensions of these results to the operator space case in Chapter 5 . 



\section{TYPE, COTYPE AND TWISTED SUMS INDUCED BY COMPLEX INTERPOLATION}

In this chapter we adapt techniques from (CASTILLO; FERENCZI; GONZÁLEZ, 2017) to the study of triviality and singularity of twisted sums induced by complex interpolation using the concepts of type and cotype. Then we apply these results to the study of submodules of the Schatten classes and obtain nontrivial twisted sums where the spaces in the short exact sequence do not have the compact approximation property.

\subsection{Type and cotype of a Banach space}

We now give the definition of (Rademacher) type of a Banach space, introduced in (HOFFMANN-JØRGENSEN, 1975). A reference for the topic is (ALBIAC; KALTON, 2006).

Definition 3.1.1. A Banach space $X$ has type $p \in[1,2]$ if there is $C>0$ such that for every $n \in \mathbb{N}$ and every sequence $\left(x_{j}\right)_{j=1}^{n}$ of vectors of $X$ we have

$$
\mathbb{E}\left\|\sum_{j=1}^{n} \varepsilon_{j} x_{n}\right\| \leq C\left(\sum_{j=1}^{n}\left\|x_{j}\right\|^{p}\right)^{\frac{1}{p}}
$$

where the expected value is taken over all choices of signs, that is,

$$
\mathbb{E}\left\|\sum_{j=1}^{n} \varepsilon_{j} x_{n}\right\|=\frac{1}{2^{n}} \sum_{\substack{\varepsilon_{j}= \pm 1 \\ j=1, \ldots, n}}\left\|\sum_{j=1}^{n} \varepsilon_{j} x_{n}\right\|
$$

The infimum of the admissible constants $C$ in (3.1) is denoted $T_{p}^{X}$. The supremum of the types of $X$ is denoted $p_{X}$.

The restriction $p \in[1,2]$ is because no bigger type is possible ((ALBIAC; KALTON, 2006), Theorem 6.2.3).

The triangle inequality implies that every Banach space $X$ has type 1 with constant $T_{1}^{X}=1$. If a Banach space has type $p^{\prime}$ then it has type $p$ for every $1 \leq p<p^{\prime}$, since $\ell_{p} \subset \ell_{p^{\prime}}$ continuously.

Notice that type is an isomorphic invariant. Also, if a Banach space $X$ has copies of the spaces $\ell_{1}^{n}$ for every $n$ with uniform constants of isomorphism then $X$ has type at most 1 . This implies that $c_{0}$ and $\ell_{\infty}$ have type at most 1 . 
The Kahane-Khintchine inequality ((ALBIAC; KALTON, 2006), Theorem 6.2.5) implies that in the definition of type we could change

$$
\mathbb{E}\left\|\sum_{j=1}^{n} \varepsilon_{j} x_{n}\right\|
$$

by

$$
\left(\mathbb{E}\left\|\sum_{j=1}^{n} \varepsilon_{j} x_{n}\right\|^{p^{\prime}}\right)^{\frac{1}{p^{\prime}}}
$$

for any $p^{\prime} \in[1, \infty)$ (but the constant appearing on the right size of (3.1) might change).

The next result follows from the parallelogram law.

Lemma 3.1.2. Let $H$ be a Hilbert space. Then for every $n \in \mathbb{N}$ and every $x_{1}, \ldots, x_{n} \in H$ we have

$$
\mathbb{E}\left\|\sum_{j=1}^{n} \varepsilon_{j} x_{j}\right\|^{2}=\sum_{j=1}^{n}\left\|x_{j}\right\|^{2}
$$

Corollary 3.1.3. Let $H$ be a Hilbert space. Then H has type 2.

The dual concept of type is that of the cotype of a Banach space. Again, we refer to (ALBIAC; KALTON, 2006).

Definition 3.1.4. Let $q \in[2, \infty]$. A Banach space $X$ has cotype $q$ if there is a constant $K>0$ such that for every $n \in \mathbb{N}$ and every finite sequence $\left(x_{j}\right)_{j=1}^{n}$ we have

$$
\left(\sum_{j=1}^{n}\left\|x_{j}\right\|^{q}\right)^{\frac{1}{q}} \leq K \mathbb{E}\left\|\sum_{j=1}^{n} \varepsilon_{j} x_{j}\right\|
$$

where the expected value is taken over all choices of signs $\varepsilon_{j}= \pm 1$.

The best constant for the cotype $q$ of a Banach space $X$ is denoted by $C_{q}^{X}$. The infimum of the cotypes of a Banach space $X$ is denoted $q_{X}$.

Example 3.1.5. Every Banach space has cotype $\infty$ with $C_{\infty}^{X}=1$.

We have the following result due to Pisier on the duality between type and cotype:

Theorem 3.1.6 ((PISIER, 1982)). Let $X$ be a Banach space with type bigger than 1 . Then $X$ has type (respectively, cotype) $p$ if and only if $X^{*}$ has cotype (respectively, type) $p^{\prime}$, with $\frac{1}{p}+\frac{1}{p^{\prime}}=1$.

In the study of type and cotype it is useful to consider the following sequence of functions:

Definition 3.1.7. The sequence of Rademacher functions is the sequence $\left(r_{k}\right)$ where each $r_{k}:[0,1] \rightarrow \mathbb{R}$ is given by

$$
r_{k}(t)=\operatorname{sgn}\left(\sin \left(2^{k} \pi t\right)\right)
$$

They are independent and satisfy

$$
P\left(r_{k}(t)=1\right)=P\left(r_{k}(t)=-1\right)=\frac{1}{2}
$$

That is

$$
\int_{0}^{1}\left\|\sum_{k=1}^{n} r_{k}(t) x_{k}\right\|_{X} d t=\mathbb{E}\left\|\sum_{k=1}^{n} \varepsilon_{k} x_{k}\right\|_{X}
$$

We will now discuss how type behaves under interpolation. 
Lemma 3.1.8. Let $G$ be a domain conformally equivalent to the disk and let $\mathscr{X}=\left\{X_{z}: z \in\right.$ $\partial G ; X\}$ be an interpolation family with containing space $\mathscr{U}$ and containing function $k$. Let $p$ be a measurable function on $\partial G$ with $1 \leq p \leq \infty$. Then the family $\mathscr{Y}=\left\{\ell_{p(z)}\left(X_{z}\right): z \in \partial G ; c_{00}(X)\right\}$ $i$ an interpolation family with containing space $\ell_{\infty}(\mathscr{U})$ and containing function $k$. Furthermore, we have norm 1 inclusions

$$
\ell_{p\left(z_{0}\right)}\left(X_{\left\{z_{0}\right\}}\right) \subset\left(\ell_{p(z)}\left(X_{z}\right)\right)_{\left\{z_{0}\right\}}
$$

and

$$
\ell_{p\left(z_{0}\right)}\left(X_{\left[z_{0}\right]}\right) \subset\left(\ell_{p(z)}\left(X_{z}\right)\right)_{\left[z_{0}\right]}
$$

where

Proof. We may suppose that $G=\mathbb{D}$.

$$
\frac{1}{p\left(z_{0}\right)}=\int_{\partial \mathbb{G}} \frac{1}{p(z)} d \mu_{z_{0}}(z)<\infty
$$

Since we have a continuous linear inclusion $X_{z} \subset \mathscr{U}$ for every $z \in \mathbb{T}$, we have continuous linear inclusions $\ell_{p(z)}\left(X_{z}\right) \subset \ell_{p(z)}(\mathscr{U}) \subset \ell_{\infty}(\mathscr{U})$. surable.

Let $x=\left(x_{n}\right) \in c_{00}(X)$. Then the function $t \mapsto\|x\|_{\ell_{p\left(e^{i t}\right)}\left(X_{e^{i t}}\right)}=\left(\sum\left\|x_{n}\right\|_{X_{e^{i t}}^{p\left(e^{i t}\right)}}^{\frac{1}{p\left(e^{i t}\right)}}\right.$ is mea-

Suppose $x$ has support contained in $\{1, \ldots, N\}$. Then

$$
\begin{aligned}
\int_{0}^{2 \pi} \log ^{+}\|x\|_{\ell_{p(z)}\left(X_{z}\right)} P_{z_{0}}\left(e^{i t}\right) d t & \leq \int_{0}^{2 \pi} \log ^{+}\|x\|_{\ell_{1}\left(X_{z}\right)} P_{z_{0}}\left(e^{i t}\right) d t \\
& \leq \int_{0}^{2 \pi} \log \left(1+\|x\|_{\ell_{1}\left(X_{e^{i t}}\right)}\right) P_{z_{0}}\left(e^{i t}\right) d t \\
& \leq \int_{0}^{2 \pi} \log \left(N+\|x\|_{\ell_{1}\left(X_{e^{i t}}\right)}\right) P_{z_{0}}\left(e^{i t}\right) d t \\
& =\int_{0}^{2 \pi} \log \left(\sum_{j=1}^{N}\left(1+\left\|x_{j}\right\|_{X_{e^{i t}}}\right)\right) P_{z_{0}}\left(e^{i t}\right) d t \\
& =\int_{0}^{2 \pi} \prod_{j=1}^{N} \log \left(1+\left\|x_{j}\right\|_{X_{e^{i t}}}\right) P_{z_{0}}\left(e^{i t}\right) d t \\
& \leq \int_{0}^{2 \pi} \prod_{j=1}^{N}\left(\log 2+\log ^{+}\left\|x_{j}\right\|_{X_{e^{i t}}}\right) P_{z_{0}}\left(e^{i t}\right) d t \\
& <\infty
\end{aligned}
$$

If $x \in c_{00}(X)$ and $z \in \mathbb{T}$, then we have

$$
\|x\|_{\ell_{\infty}(\mathscr{U})} \leq k(z)\|x\|_{\ell_{\infty}\left(X_{z}\right)} \leq k(z)\|x\|_{\ell_{p(z)}\left(X_{z}\right)}
$$

So $\left\{\ell_{p(z)}\left(X_{z}\right): z \in \partial G ; c_{00}(X)\right\}$ is an interpolation family with containing space $\ell_{\infty}(\mathscr{U})$ and containing function $k$.

Let $x=\left(x_{j}\right) \in c_{00}(X)$, and suppose that the support of $x$ is contained in $\{1, \ldots, N\}$ and $\|x\|_{\ell_{p\left(z_{0}\right)}\left(X_{\left\{z_{0}\right\}}\right)}=1$. Given $\varepsilon>0$, let $g_{j} \in \mathscr{G}(\mathscr{X})$ be such that $g_{j}\left(z_{0}\right)=x_{j}$ and $\left\|g_{j}\right\| \leq$ $(1+\varepsilon)\left\|x_{j}\right\|_{\left\{z_{0}\right\}}, 1 \leq j \leq N$.

Let

$$
g(z)=\sum_{j=1}^{N} g_{j}(z)\left\|x_{j}\right\|_{\left\{z_{0}\right\}}^{\frac{p\left(z_{0}\right)}{p(z)}-1} e_{j}
$$


Then $g \in \mathscr{G}(\mathscr{Y}), g\left(z_{0}\right)=x$ and $\|g\| \leq 1+\varepsilon$. Therefore, we have a norm 1 inclusion

$$
\ell_{p\left(z_{0}\right)}\left(X_{\left\{z_{0}\right\}}\right) \subset\left(\ell_{p(z)}\left(X_{z}\right)\right)_{\left\{z_{0}\right\}}
$$

For the inclusion

$$
\ell_{p\left(z_{0}\right)}\left(X_{\left[z_{0}\right]}\right) \subset\left(\ell_{p(z)}\left(X_{z}\right)\right)_{\left[z_{0}\right]}
$$

we do the same as before but use functions in $\mathscr{F}(\mathscr{X})$.

In the following proposition, we work with $L_{1}=L_{1}[0,1]$.

Lemma 3.1.9. Let $\mathscr{X}=\left\{X_{z}: z \in \partial G ; X\right\}$ be an interpolation family with containing space $\mathscr{U}$ and containing function $k$.

Let $\operatorname{sim}(X)$ be the space of measurable simple functions on $[0,1]$ with values in $X$.

Then the family $\mathscr{Y}=\left\{L_{1}\left(X_{z}\right): z \in \partial G ; \operatorname{sim}(X)\right\}$ is an interpolation family with containing space $L_{1}(\mathscr{U})$ and containing function $k$. Furthermore, we have the identity

$$
\left(L_{1}\left(X_{z}\right)\right)_{\left\{z_{0}\right\}}=L_{1}\left(X_{\left\{z_{0}\right\}}\right)
$$

and the norm 1 inclusion

Proof. We may suppose that $G=\mathbb{D}$.

$$
L_{1}\left(X_{\left[z_{0}\right]}\right) \subset\left(L_{1}\left(X_{z}\right)\right)_{\left[z_{0}\right]}
$$

It is clear that $L_{1}(\mathscr{U})$ is a containing space for $\mathscr{Y}$. Let $f \in \operatorname{sim}(X), f=\sum \chi_{A_{j}} x_{j}$, where $A_{j} \subset[0,1]$ are disjoint and $x_{j} \in X$. Then

$$
\begin{aligned}
\|f\|_{L_{1}\left(X_{e^{i} t}\right)} & =\int_{0}^{1}\|f(s)\|_{X_{e^{i t}}} d s \\
& =\sum \Lambda\left(A_{j}\right)\left\|x_{j}\right\|_{e^{i t}}
\end{aligned}
$$

which is measurable, and this equality also implies I1.

Also, we have $\|f\|_{L_{1}(\mathscr{U})} \leq k\left(e^{i t}\right)\|f\|_{L_{1}\left(X_{e^{i t}}\right)}$, so we have I2. Now, let $g_{j} \in \mathscr{G}(\mathscr{X})$ be such that $\left\|g_{j}\right\| \leq(1+\varepsilon)\left\|x_{j}\right\|_{\left\{z_{0}\right\}}$ and $g_{j}\left(z_{0}\right)=x_{j}$. $h\left(z_{0}\right)=f$,

We have $g_{j}=\sum_{k=1}^{N_{j}} \psi_{k}^{j} x_{k}^{j}$, for some $\psi_{k}^{j} \in N^{+}$and $x_{k}^{j} \in X$. Let $h=\sum \chi_{A_{j}} g_{j}$. Then we have

$$
\begin{aligned}
h & =\sum_{j} \chi_{A_{j}} g_{j} \\
& =\sum_{j} \chi_{A_{j}} \sum_{k=1}^{N_{j}} \psi_{k}^{j} x_{k}^{j} \\
& =\sum_{j, k} \psi_{k}^{j} \chi_{A_{j}} x_{k}^{j}
\end{aligned}
$$

and

$$
\begin{aligned}
\|h(z)\|_{L_{1}\left(X_{z}\right)} & =\left\|\sum_{j} \chi_{A_{j}} g_{j}\right\|_{L_{1}\left(X_{z}\right)} \\
& =\sum_{j} \Lambda\left(A_{j}\right)\left\|g_{j}\right\|_{L_{1}\left(X_{z}\right)}
\end{aligned}
$$




$$
\leq(1+\varepsilon)\|f\|_{L_{1}\left(X_{\left\{z_{0}\right\}}\right)}
$$

so that $h \in \mathscr{G}(\mathscr{Y})$ and $\|f\|_{\left(L_{1}\left(X_{z}\right)\right)_{\left\{z_{0}\right\}}} \leq\|f\|_{L_{1}\left(X_{\left\{z_{0}\right\}}\right)}$.

On the other hand, given $h \in \mathscr{G}(\mathscr{Y})$ such that $h\left(z_{0}\right)=f$, then for every $s \in[0,1]$ we have $h().(s) \in \mathscr{G}(\mathscr{X})$ and $h\left(z_{0}\right)(s)=f(s)$. Therefore, by Proposition 2.3.68,

$$
\begin{aligned}
\|f\|_{L_{1}\left(X_{\left\{z_{0}\right\}}\right)} & =\int_{0}^{1}\|f(s)\|_{X_{\left\{z_{0}\right\}}} d s \\
& \leq \int_{0}^{1} \int_{\mathbb{T}}\|h(z)(s)\|_{X_{z}} P_{z_{0}}(z) d z d s \\
& =\int_{\mathbb{T}} \int_{0}^{1}\|h(z)(s)\|_{X_{z}} P_{z_{0}}(z) d s d z \\
& =\int_{\mathbb{T}}\|h(z)\|_{L_{1}\left(X_{z}\right)} P_{z_{0}}(z) d z \\
& \leq\|h\|
\end{aligned}
$$

so that $\|f\|_{L_{1}\left(X_{\left\{z_{0}\right\}}\right)} \leq\|f\|_{\left(L_{1}\left(X_{z}\right)\right)_{\left\{z_{0}\right\}}}$.

To get the inclusion

$$
L_{1}\left(X_{\left[z_{0}\right]}\right) \subset\left(L_{1}\left(X_{z}\right)\right)_{\left[z_{0}\right]}
$$

we use the same argument we used to obtain the inclusion for $\left\{z_{0}\right\}$, but taking functions $f_{j} \in \mathscr{F}(\mathscr{X})$ and approximating by functions in $\mathscr{G}(\mathscr{X})$.

The following result may be found in (KALTON, 1989) in the more general context of interpolation of families of quasi-Banach spaces.

Theorem 3.1.10. [(KALTON, 1989), Theorem 5] Let $G$ be a domain conformally equivalent to the disk, and let $\left\{X_{z}: z \in \partial G ; X\right\}$ be an interpolation family with containing space $\mathscr{U}$, and let $p: \partial G \rightarrow[1,2]$ and $C: \partial G \rightarrow[1, \infty]$ be measurable functions such that $X_{z}$ has type $p(z)$ with constant $C(z)$ for almost every $z \in \partial G$ and

$$
\begin{gathered}
\int_{\partial G} \frac{1}{p(z)} d z<\infty \\
\int_{\partial G} \log C(z) d z<\infty
\end{gathered}
$$

Then for every $z_{0} \in G, X_{\left\{z_{0}\right\}}$ has type $p\left(z_{0}\right)$ with constant $C\left(z_{0}\right)$, where

$$
\begin{gathered}
\frac{1}{p\left(z_{0}\right)}=\int_{\partial G} \frac{1}{p(z)} d \mu_{z_{0}}(z)<\infty \\
C\left(z_{0}\right)=\exp \int_{\partial G} \log C(z) d \mu_{z_{0}}(z)
\end{gathered}
$$

Proof. For each $n$, consider the interpolation families $\left\{\ell_{p(z)}\left(X_{z}\right), z \in \partial G ; c_{00}(X)\right\}$ and $\left\{L_{1}\left(X_{z}\right), z \in\right.$ $\partial G ; \operatorname{sim}(X)\}$.

Let $\left(r_{j}\right)$ be the Rademacher functions and let $T^{n}: \ell_{\infty}^{n}(\mathscr{U}) \rightarrow L_{1}(\mathscr{U})$ be given by

$$
T^{n}\left(\left(x_{j}\right)_{j}\right)=\sum_{j=1}^{n} r_{j}(.) x_{j}
$$

Then $T^{n}$ maps $c_{00}(X)$ into $\operatorname{sim}(X)$. 
Notice that $\log C(z)$ is absolutely integrable, since it is nonnegative.

By the hypothesis we have for a. e. $z \in \mathbb{T}$ and every $x \in c_{00}(X)$ that $\left\|T^{n} x\right\|_{L_{1}\left(X_{Z}\right)} \leq$ $C(z)\|x\|_{\ell_{p(z)}\left(X_{z}\right)}$, so by Theorem 2.3.58 $T^{n}$ is bounded from $\left(\ell_{p(z)}\left(X_{z}\right)\right)_{\left\{z_{0}\right\}}$ into $\left(L_{1}\left(X_{z}\right)\right)_{\left\{z_{0}\right\}}$ with norm at most $C\left(z_{0}\right)$. theorem.

Using the inclusions and identifications from Lemmata 3.1.8 and 3.1.9 we obtain the

As a particular case, we have the following classical result:

Corollary 3.1.11. Let $\left(X_{0}, X_{1}\right)$ be a compatible couple such that $X_{0}$ has type $p_{0}$ and $X_{1}$ has type $p_{1}$. For $\theta \in(0,1)$ let $\frac{1}{p_{\theta}}=\frac{1-\theta}{p_{0}}+\frac{\theta}{p_{1}}$. Then the space $X_{\theta}$ has type $p_{\theta}$.

Example 3.1.12. The spaces $\ell_{p}, p \in[1, \infty)$, have type $\min \{p, 2\}$. Let us show this fact for $p \in[1,2]$. Since $\ell_{p}=\left(\ell_{2}, \ell_{1}\right)_{\theta}, \theta=\frac{2-p}{p}$, it follows from Corollary 3.1.11 that $\ell_{p}$ has type $p_{\theta}$ given by

$$
\frac{1}{p_{\theta}}=\frac{1-\frac{2-p}{p}}{2}+\frac{2-p}{p}=\frac{1}{p}
$$

Therefore, $\ell_{p}$ has type $p$, for $p \in[1,2]$.

Actually, $p_{\ell_{p}}=p$ for $p \in[1,2]$. Indeed, since for any choice of signs $\left(\varepsilon_{j}\right)$ we have

$$
\left\|\sum_{j=1}^{n} \varepsilon_{j} e_{j}\right\|_{p}=n^{\frac{1}{p}}
$$

if $\ell_{p}$ had type $p^{\prime}>p$ we would have

$$
n^{\frac{1}{p}} \leq C_{p^{\prime}}^{X} n^{\frac{1}{p^{\prime}}}
$$

for every $n$, which is impossible.

For $2<p<\infty$, the proof that $\ell_{p}$ has type 2 uses Kahane's inequality. A proof may be found in (ALBIAC; KALTON, 2006).

Example 3.1.13. As seen in Example 3.1.12, for $1 \leq p \leq 2$ we have that the best type of the space $\ell_{p}$ is $p$. Theorem 3.1.6 implies that the best cotype of the space $\ell_{q}$ for $2 \leq q<\infty$ is $q$. Also, it may be verified that the best cotype for $\ell_{\infty}$ is $\infty$.

\subsection{Type and twisted sums induced by interpolation}

We now obtain estimates relating the types of the spaces in the interpolation family and the triviality and singularity of the induced twisted sum.

Definition 3.2.1. Let $G$ be a domain conformally equivalent to the disk, and let $\left\{X_{z}: z \in \partial G ; X\right\}$ be an interpolation family. If $z_{0} \in G$, by $\|\cdot\|_{z_{0}}$ (or simply $\|$.$\| \| , if the context is clear) we denote$ the following norm on $\mathscr{F}$ :

$$
\|f\|_{z_{0}}=\int_{\partial G}\|f(z)\|_{z} d \mu_{z_{0}}(z)
$$

From Propositions 2.3.68 and 2.3.73, if the family is admissible at $z_{0}$ we have that for every $x \in X_{z_{0}}$

$$
\|x\|_{z_{0}}=\inf \left\{\|f \mid\|_{z_{0}}: f \in \mathscr{F}, f\left(z_{0}\right)=x\right\}
$$


Proposition 3.2.2. Let $G$ be a domain conformally equivalent to the disk and let $\left\{X_{z}: z \in \partial G ; X\right\}$ be an interpolation family admissible at $z_{0} \in G$. Then $\delta_{z_{0}}^{\prime}: \operatorname{ker}\left(\delta_{z_{0}}\right) \rightarrow X_{z_{0}}$ is bounded when we consider $\operatorname{ker}\left(\delta_{z_{0}}\right)$ normed by $\|\cdot\| \|_{z_{0}}$.

Proof. Let $f \in \operatorname{ker}\left(\delta_{z_{0}}\right)$ and $\varphi: G \rightarrow \mathbb{D}$ be a conformal map with $\varphi\left(z_{0}\right)=0$. Since the family is admissible at $z_{0}$, the function $h=\frac{f}{\varphi}$ is in $\mathscr{F}$, and $h\left(z_{0}\right)=\frac{f^{\prime}\left(z_{0}\right)}{\varphi^{\prime}\left(z_{0}\right)}$. Therefore:

$$
\begin{aligned}
\left\|f^{\prime}\left(z_{0}\right)\right\|_{z_{0}} & \leq\left|\varphi^{\prime}\left(z_{0}\right)\right|\|h\|_{z_{0}} \\
& =\left|\varphi^{\prime}\left(z_{0}\right)\right| \int_{\partial G}\left\|\frac{f(z)}{\varphi(z)}\right\|_{z} d \mu_{z_{0}}(z) \\
& =\left|\varphi^{\prime}\left(z_{0}\right)\right|\|f\|
\end{aligned}
$$

so that $\left\|\delta_{z_{0}}^{\prime}\right\| \leq\left|\varphi^{\prime}\left(z_{0}\right)\right|$.

Another relation between $\|\cdot \cdot\|_{z_{0}}$ and $\|\cdot\|_{\mathscr{F}}$ that will be useful is the following: since $\|\cdot\|_{z_{0}} \leq\|\cdot\|_{\mathscr{F}}$ it follows that if $B_{z_{0}}: X_{z_{0}} \rightarrow \mathscr{F}$ is a homogeneous section for $\delta_{z_{0}}$ bounded with respect to $\|\cdot\|_{\mathscr{F}}$ then $B_{z_{0}}$ is also bounded with respect to $\|\cdot\|_{z_{0}}$.

From now on we fix an interpolation family $\left\{X_{z}: z \in \partial G ; X\right\}$ with containing space $\mathscr{U}$ and we suppose that it is admissible at $z_{0} \in G$. We also fix a bounded homogeneous section $B_{z_{0}}: X_{z_{0}} \rightarrow \mathscr{F}$ for $\delta_{z_{0}}$, and denote by $\Omega_{z_{0}}=\delta_{z_{0}}^{\prime} B_{z_{0}}: X_{z_{0}} \rightarrow \mathscr{U}$ the $\Omega$-operator.

The following known lemma follows by definition.

Lemma 3.2.3. Suppose that $W$ is a closed subspace of $X_{z_{0}}$ and that $\left.\Omega_{z_{0}}\right|_{W}$ is trivial. Then there is a linear map $\Lambda: W \rightarrow \mathscr{U}$ such that $\left.\Omega_{z_{0}}\right|_{W}-\Lambda: W \rightarrow X_{z_{0}}$ is bounded.

Proof. Let $L_{z_{0}}: X_{z_{0}} \rightarrow \mathscr{F}$ be a linear section for $\delta_{z_{0}}$. Then $F_{z_{0}}=\delta_{z_{0}}^{\prime} \circ\left(B_{z_{0}}-L_{z_{0}}\right)$ is a quasilinear map that defines the twisted sum $d_{\Omega_{z_{0}}} X_{z_{0}}$, and triviality of $\left.\Omega_{z_{0}}\right|_{W}$ is the same as triviality of $\left.F_{z_{0}}\right|_{W}$. This means that there is a linear map $L: W \rightarrow X_{z_{0}}$ such that $\left.F_{z_{0}}\right|_{W}-L: W \rightarrow X_{z_{0}}$ is bounded.

Let $\Lambda=\left.\left(\delta_{z_{0}}^{\prime} \circ L_{z_{0}}\right)\right|_{W}-L: X_{z_{0}} \rightarrow \mathscr{U}$. Then $\left.\Omega_{z_{0}}\right|_{W}-\Lambda=\left.F_{z_{0}}\right|_{W}-L: W \rightarrow X_{z_{0}}$ is bounded.

In (CASTILLO; FERENCZI; GONZÁLEZ, 2017) the following estimator is used for a Köthe function space $X$ :

$$
M_{X}(n)=\sup \left\{\left\|x_{1}+\ldots+x_{n}\right\|\right\}
$$

where the supremum is taken over $x_{1}, \ldots, x_{n}$ in the unit ball of $X$ with disjoint supports. So we can change the signs of the functios $x_{j}, j=1, \ldots, n$, without changing the value of $M_{X}(n)$. This suggests the following estimator:

Definition 3.2.4. Let $X$ be a Banach space and $n \in \mathbb{N}$. We define

$$
\beta_{n}(X)=\sup \mathbb{E}\left\|\sum_{j=1}^{n} \varepsilon_{j} x_{j}\right\|
$$

where the supremum is over all sequences $\left(x_{j}\right)_{j=1}^{n}$ with $\left\|x_{j}\right\| \leq 1$ for every $j=1, \ldots, n$.

Notice that $\beta_{n}(X)$ is nothing more than the norm of $T: \ell_{\infty}^{n}(X) \rightarrow L_{1}(X)$ given by

$$
T\left(x_{j}\right)_{j=1}^{n}=\sum_{j=1}^{n} r_{j}(.) x_{j}
$$

Also, if $X$ is a Köthe function space then $\beta_{n}(X) \leq M_{X}(n)$ for every $n$.

For every Banach space $X$ we have $\beta_{1}(X)=1$ and for every $n \in \mathbb{N}$ we have $\beta_{n+1}(X) \geq$ $\beta_{n}(X)$. 
Lemma 3.2.5. Let $X$ be a Banach space, $n \in \mathbb{N}$, and $x_{1}, \ldots, x_{n} \in X$. Then

$$
\mathbb{E}\left\|\sum_{j=1}^{n} \varepsilon_{j} x_{j}\right\| \leq \beta_{n}(X) \sup _{j}\left\|x_{j}\right\|
$$

Proof. If $\sup _{j}\left\|x_{j}\right\|=0$, then the result is clear. If it is not zero, let

$$
y_{j}=\frac{x_{j}}{\sup _{j}\left\|x_{j}\right\|}
$$

$j=1, \ldots, n$. Then $\left\|y_{j}\right\| \leq 1$ for every $j$, and therefore

$$
\mathbb{E}\left\|\sum_{j=1}^{n} \varepsilon_{j} x_{j}\right\| \leq \beta_{n}(X)
$$

which implies

$$
\mathbb{E}\left\|\sum_{j=1}^{n} \varepsilon_{j} x_{j}\right\| \leq \beta_{n}(X) \sup _{j}\left\|x_{j}\right\|
$$

Theorem 3.2.6 (Maurey-Pisier, (MAUREY; PISIER, 1976), Théorème 2.3). Let X be an infinite dimensional Banach space. Then, for every $n \in \mathbb{N}$ and every $\varepsilon>0$ there are vectors $x_{1}, \ldots, x_{n} \in X$ of norm at most 1 such that for every $a_{1}, \ldots, a_{n} \in \mathbb{R}$ we have

$$
\frac{1}{1+\varepsilon}\left(\sum_{j=1}^{n}\left|a_{j}\right|^{p_{X}}\right)^{\frac{1}{p_{X}}} \leq\left\|\sum_{j=1}^{n} a_{j} x_{j}\right\| \leq\left(\sum_{j=1}^{n}\left|a_{j}\right|^{p_{X}}\right)^{\frac{1}{p_{X}}}
$$

Lemma 3.2.7. Let $X$ be an infinite dimensional Banach space with type $p=p_{X}$. Then for every $n \geq 1$ we have $n^{\frac{1}{p}} \leq \beta_{n}(X) \leq T_{p}^{X} n^{\frac{1}{p}}$.

Proof. Let $x_{1}, \ldots, x_{n} \in X$ with norm less or equal to 1 . Then, since $X$ has type $p$, we have

$$
\begin{aligned}
\mathbb{E}\left\|\sum_{j=1}^{n} \varepsilon_{j} x_{j}\right\| & \leq T_{p}^{X}\left(\sum_{j=1}^{n}\left\|x_{j}\right\|^{p}\right)^{\frac{1}{p}} \\
& \leq T_{p}^{X} n^{\frac{1}{p}}
\end{aligned}
$$

so that $\beta_{n}(X) \leq T_{p}^{X} n^{\frac{1}{p}}$. For the other inequality, by Maurey-Pisier's Theorem 3.2.6, given $\varepsilon>0$ there are $x_{1}, \ldots, x_{n} \in X$ of norm at most 1 such that for every $a_{1}, \ldots, a_{n} \in \mathbb{R}$ we have

$$
\frac{1}{1+\varepsilon}\left(\sum_{j=1}^{n}\left|a_{j}\right|^{p}\right)^{\frac{1}{p}} \leq\left\|\sum_{j=1}^{n} a_{j} x_{j}\right\| \leq\left(\sum_{j=1}^{n}\left|a_{j}\right|^{p}\right)^{\frac{1}{p}}
$$

In particular

$$
\frac{1}{1+\varepsilon} n^{\frac{1}{p}} \leq\left\|\sum_{j=1}^{n} \varepsilon_{j} x_{j}\right\| \leq n^{\frac{1}{p}}
$$

for every choice of signs $\varepsilon_{j}= \pm 1,1 \leq j \leq n$. It follows that

$$
\frac{1}{1+\varepsilon} n^{\frac{1}{p}} \leq \mathbb{E}\left\|\sum_{j=1}^{n} \varepsilon_{j} x_{j}\right\| \leq \beta_{n}(X)
$$

and since $\varepsilon$ was arbitrary, we obtain $n^{\frac{1}{p}} \leq \beta_{n}(X)$. 
Notation 3.2.8. If $X$ is a Banach space, by $B_{X}$ we denote the closed unit ball in $X$.

We now obtain a series of estimates. The principle behind the results in (CASTILLO; FERENCZI; GONZÁLEZ, 2017) and the ones we will present here is the following: let $\alpha$ be a parameter of Banach spaces (for example, type). If the spaces being interpolated are not similar with respect to $\alpha$ and if $\alpha\left(X_{\theta}\right)$ behaves exactly as the interpolation of the parameters then the twisted sum is nontrivial. Furthermore, if $\alpha(W)=\alpha\left(X_{\theta}\right)$ for any closed infinite dimensional subspace $W$ of $X_{\theta}$ then the twisted sum is singular.

Lemma 3.2.9. Let $X=X_{z_{0}}$ be an interpolation space and $\Omega=\Omega_{z_{0}}$ be induced by interpolation. Let $W$ be a closed subspace of $X$ such that $\left.\Omega\right|_{W}$ is trivial. Then there is a constant $K_{1}$ such that for every finite sequence $\left(w_{j}\right)_{j=1}^{n} \subset B_{W}$ we have

$$
\mathbb{E}\left\|\Omega\left(\sum_{j=1}^{n} \varepsilon_{j} w_{j}\right)-\sum_{j=1}^{n} \varepsilon_{j} \Omega\left(w_{j}\right)\right\| \leq K_{1} \beta_{n}(X)
$$

Proof. Let $\mathscr{U}$ be the containing space of the family defining $X$. Then $\Omega_{z_{0}}: X \rightarrow \mathscr{U}$. Take $\Lambda: W \rightarrow \mathscr{U}$ such that $\left.\Omega\right|_{W}-\Lambda: W \rightarrow X$ is bounded (Lemma 3.2.3).

Then for every choice of signs $\varepsilon_{j}= \pm 1$ we have

$$
\begin{aligned}
\left\|\Omega\left(\sum_{j=1}^{n} \varepsilon_{j} w_{j}\right)-\sum_{j=1}^{n} \varepsilon_{j} \Omega\left(w_{j}\right)\right\| \leq & \left\|\Omega\left(\sum_{j=1}^{n} \varepsilon_{j} w_{j}\right)-\Lambda\left(\sum_{j=1}^{n} \varepsilon_{j} w_{j}\right)\right\| \\
& +\left\|\sum_{j=1}^{n} \varepsilon_{j} \Lambda\left(w_{j}\right)-\sum_{j=1}^{n} \varepsilon_{j} \Omega\left(w_{j}\right)\right\|
\end{aligned}
$$

But

$$
\begin{aligned}
\mathbb{E}\left\|\Omega\left(\sum_{j=1}^{n} \varepsilon_{j} w_{j}\right)-\Lambda\left(\sum_{j=1}^{n} \varepsilon_{j} w_{j}\right)\right\| & \leq\|\Omega-\Lambda\| \mathbb{E}\left\|\sum_{j=1}^{n} \varepsilon_{j} w_{j}\right\| \\
& \leq\|\Omega-\Lambda\| \beta_{n}(X)
\end{aligned}
$$

and

$$
\begin{aligned}
\mathbb{E}\left\|\sum_{j=1}^{n} \varepsilon_{j} \Lambda\left(w_{j}\right)-\sum_{j=1}^{n} \varepsilon_{j} \Omega\left(w_{j}\right)\right\| & =\mathbb{E}\left\|\sum_{j=1}^{n} \varepsilon_{j}(\Lambda-\Omega)\left(w_{j}\right)\right\| \\
& \leq \beta_{n}(X) \sup _{j}\left\|(\Lambda-\Omega)\left(w_{j}\right)\right\| \\
& \leq\|\Omega-\Lambda\| \beta_{n}(X)
\end{aligned}
$$

So that

$$
\mathbb{E}\left\|\Omega\left(\sum_{j=1}^{n} \varepsilon_{j} w_{j}\right)-\sum_{j=1}^{n} \varepsilon_{j} \Omega\left(w_{j}\right)\right\| \leq K_{1} \beta_{n}(X)
$$

with $K_{1}=2\|\Omega-\Lambda\|$.

Proposition 3.2.10. Let $\left\{X_{z}: z \in \partial G ; X\right\}$ be an interpolation family admissible at $z_{0}$ and suppose that for some $n \in \mathbb{N}$ there is a measurable function $f: \partial G \rightarrow[0, \infty)$ such that $\beta_{n}\left(X_{z}\right) \leq f(z)$ for a. e. $z \in \partial G$. Then:

$$
\beta_{n}\left(X_{z_{0}}\right) \leq \exp \int_{\partial G} \log f(z) d \mu_{z_{0}}(z)
$$


Proof. Recall that $\beta_{n}(X)$ is the norm of $T: \ell_{\infty}^{n}(X) \rightarrow L_{1}(X)$ given by

$$
T\left(x_{j}\right)_{j=1}^{n}=\sum_{j=1}^{n} r_{j}(.) x_{j}
$$

From this, Theorem 2.3.58 and Lemmata 3.1.8 and 3.1.9 the result follows.

A specific case in which we will be interested is the following:

Definition 3.2.11. Let $\left\{X_{z}: z \in \partial \mathbb{S} ; X\right\}$ be an interpolation family. We denote by

$$
\beta_{n, j}=\underset{z \in \mathbb{S}_{j}}{\operatorname{ess} \sup _{n}} \beta_{n}\left(X_{z}\right)
$$

$j=0,1$, whenever it is well-defined.

Observation 3.2.12. Whenever we use $\beta_{n, j}$ we will be implicitly assuming that it is well-defined.

Proposition 3.2.13. Let $\left\{X_{z}: z \in \partial \mathbb{S} ; X\right\}$ be an interpolation family admissible at $z_{0}$. Then $\beta_{n}\left(X_{z_{0}}\right) \leq \beta_{n, 0}^{1-\operatorname{Re}\left(z_{0}\right)} \beta_{n, 1}^{\operatorname{Re}\left(z_{0}\right)}$.

Proof. Let $f: \partial \mathbb{S} \rightarrow[0, \infty)$ be given by $f(j+i t)=\beta_{n, j}, j=0,1, t \in \mathbb{R}$.

Then

$$
\int_{\partial \mathbb{S}} \log f(z) d \mu_{z_{0}}(z)=\left(1-\operatorname{Re}\left(z_{0}\right)\right) \log \beta_{n, 0}+\operatorname{Re}\left(z_{0}\right) \log \beta_{n, 1}
$$

and by Proposition 3.2.10 we have

$$
\beta_{n}\left(X_{z_{0}}\right) \leq \exp \left(\left(1-\operatorname{Re}\left(z_{0}\right)\right) \log \beta_{n, 0}+\operatorname{Re}\left(z_{0}\right) \log \beta_{n, 1}\right)=\beta_{n, 0}^{1-\operatorname{Re}\left(z_{0}\right)} \beta_{n, 1}^{\operatorname{Re}\left(z_{0}\right)}
$$

Lemma 3.2.14. Let $\left\{X_{z}: z \in \partial G ; X\right\}$ be an interpolation family admissible at $z_{0}$ and suppose that for some $n \in \mathbb{N}$ there is an absolutely log-integrable function $f_{n}: \partial G \rightarrow[0, \infty)$ such that $\beta_{n}\left(X_{z}\right) \leq f_{n}(z)$ for a. e. $z \in \partial G$. Let $v_{n}$ be a harmonic conjugate of

$$
u_{n}\left(z_{0}\right)=\int_{\partial G} \log f_{n}(z) d \mu_{z_{0}}(z)
$$

and define $\beta_{n}\left(f_{n}, z\right)=e^{u_{n}(z)+i v_{n}(z)}$ (i. e., $\beta_{n}\left(f_{n},.\right)$ is an outer function associated to $u_{n}$. We hide the dependence on $\left.v_{n}\right)$. Then there is $K_{2}>0$ such that for every finite sequence $\left(x_{j}\right)_{j=1}^{n} \subset B_{X_{z_{0}}}$ we have

$$
\mathbb{E}\left\|\sum_{j=1}^{n} \varepsilon_{j} x_{j} \frac{-\beta_{n}^{\prime}\left(f_{n}, z_{0}\right)}{\beta_{n}\left(f_{n}, z_{0}\right)}-\Omega_{z_{0}}\left(\sum_{j=1}^{n} \varepsilon_{j} x_{j}\right)+\sum_{j=1}^{n} \Omega_{z_{0}}\left(\varepsilon_{j} x_{j}\right)\right\| \leq K_{2} e^{u_{n}\left(z_{0}\right)}
$$

Also, $K_{2}$ does not depend on $n, f_{n}$ or $v_{n}$.

Proof. For every $\varepsilon=\left(\varepsilon_{j}\right) \in\{-1,1\}^{n}$ consider the function

$$
F_{\varepsilon}(z)=\frac{\sum_{j=1}^{n} \varepsilon_{j} B_{z_{0}}\left(x_{j}\right)(z)}{\beta_{n}\left(f_{n}, z\right)}
$$

Notice that $\beta_{n}\left(f_{n}, z\right)$ has modulus bigger than or equal to $e$ on $G$ by the minimum modulus principle since $f_{n} \geq 1$ a. e. So $\frac{1}{\beta_{n}\left(f_{n}, .\right)} \in H^{\infty}(G)$. This implies that $F_{\varepsilon} \in \mathscr{F}$ (this may be seem by approximating each $B_{z_{0}}\left(x_{z}\right)$ by functions in $\left.\mathscr{G}\right)$. 
We have $\mathbb{E}\left\|F_{\varepsilon}\right\| \leq\left\|B_{z_{0}}\right\|$. Indeed

$$
\begin{aligned}
\mathbb{E}\left\|F_{\varepsilon}\right\| & =\mathbb{E} \int_{\partial \mathbb{S}}\left\|F_{\varepsilon}(z)\right\|_{z} d \mu_{z_{0}}(z) \\
& =\int_{\partial \mathbb{S}} \mathbb{E}\left\|\frac{\sum_{j=1}^{n} B_{z_{0}}\left(x_{j}\right)(z)}{f_{n}(z)}\right\|_{z} d \mu_{z_{0}}(z) \\
& \leq \int_{\partial \mathbb{S}} \mathbb{E}\left\|\frac{\sum_{j=1}^{n} B_{z_{0}}\left(x_{j}\right)(z)}{\beta_{n}\left(X_{z}\right)}\right\| \|_{z} d \mu_{z_{0}}(z) \\
& \leq \int_{\partial \mathbb{S}} \sup \left\|B_{z_{0}}\left(x_{j}\right)(z)\right\|_{z} d \mu_{z_{0}}(z) \\
& \leq \int_{\partial \mathbb{S}} \sup _{j}\left\|B_{z_{0}}\left(x_{j}\right)\right\| d \mu_{z_{0}} \\
& \leq \int_{\partial \mathbb{S}}\left\|B_{z_{0}}\right\| d \mu_{z_{0}} \\
& =\left\|B_{z_{0}}\right\|
\end{aligned}
$$

Also

$$
F_{\varepsilon}\left(z_{0}\right)=\frac{\sum_{j=1}^{n} \varepsilon_{j} x_{j}}{\beta_{n}\left(f_{n}, z_{0}\right)}
$$

and

$$
F_{\varepsilon}^{\prime}\left(z_{0}\right)=\frac{\sum_{j=1}^{n} \varepsilon_{j} \Omega_{z_{0}}\left(x_{j}\right)}{\beta_{n}\left(f_{n}, z_{0}\right)}-\frac{\beta_{n}^{\prime}\left(f_{n}, z_{0}\right)}{\beta_{n}\left(f_{n}, z_{0}\right)} F_{\varepsilon}\left(z_{0}\right)
$$

Then

$$
\begin{array}{r}
\delta_{z_{0}}^{\prime}\left(\beta_{n}\left(f_{n}, z_{0}\right) F_{\varepsilon}\right)-\Omega_{z_{0}}\left(\sum_{j=1}^{n} \varepsilon_{j} x_{j}\right) \\
=\sum_{j=1}^{n} \varepsilon_{j} \Omega_{z_{0}}\left(x_{j}\right)-\frac{\beta_{n}^{\prime}\left(f_{n}, z_{0}\right)}{\beta_{n}\left(f_{n}, z_{0}\right)} \sum_{j=1}^{n} \varepsilon_{j} x_{j}-\Omega_{z_{0}}\left(\sum_{j=1}^{n} \varepsilon_{j} x_{j}\right)
\end{array}
$$

Notice that $\beta_{n}\left(f_{n}, z_{0}\right) F_{\varepsilon}-B_{z_{0}}\left(\sum_{j=1}^{n} \varepsilon_{j} x_{j}\right) \in \operatorname{ker} \delta_{z_{0}}$. So, by Proposition 3.2.2

$$
\begin{aligned}
& \left\|-\left(\sum_{j=1}^{n} \varepsilon_{j} x_{j}\right) \frac{\beta_{n}^{\prime}\left(f_{n}, z_{0}\right)}{\beta_{n}\left(f_{n}, z_{0}\right)}-\Omega_{z_{0}}\left(\sum_{j=1}^{n} \varepsilon_{j} x_{j}\right)+\sum_{j=1}^{n} \Omega_{z_{0}}\left(\varepsilon_{j} x_{j}\right)\right\| \\
= & \left\|\delta_{z_{0}}^{\prime}\left(\beta_{n}\left(f_{n}, z_{0}\right) F_{\varepsilon}\right)-\Omega_{z_{0}}\left(\sum_{j=1}^{n} \varepsilon_{j} x_{j}\right)\right\| \\
\leq & \left\|\delta_{z_{0}}^{\prime}\right\|\|\| \beta_{n}\left(f_{n}, z_{0}\right) F_{\varepsilon}-B_{z_{0}}\left(\sum_{j=1}^{n} \varepsilon_{j} x_{j}\right)\|\| \\
\leq & \left\|\delta_{z_{0}}^{\prime}\right\|\left(\left\|\beta_{n}\left(f_{n}, z_{0}\right) F_{\varepsilon}\right\|+\left\|B_{z_{0}}\left(\sum_{j=1}^{n} \varepsilon_{j} x_{j}\right)\right\| \|\right)
\end{aligned}
$$




$$
\leq\left\|\delta_{z_{0}}\right\|\left(\left|\beta_{n}\left(f_{n}, z_{0}\right)\right|\left\|F_{\varepsilon}\right\| \mid+\left\|B_{z_{0}}\right\|\left\|\sum_{j=1}^{n} \varepsilon_{j} x_{j}\right\|\right)
$$

Taking the expected value, using Proposition 3.2.10 and using that $\mathbb{E}\left\|F_{\mathcal{E}}\right\| \leq\left\|B_{z_{0}}\right\|$, we obtain

$$
\begin{aligned}
& \mathbb{E}\left\|-\left(\sum_{j=1}^{n} \varepsilon_{j} x_{j}\right) \frac{\beta_{n}^{\prime}\left(f_{n}, z_{0}\right)}{\beta_{n}\left(f_{n}, z_{0}\right)}-\Omega_{z_{0}}\left(\sum_{j=1}^{n} \varepsilon_{j} x_{j}\right)+\sum_{j=1}^{n} \Omega_{z_{0}}\left(\varepsilon_{j} x_{j}\right)\right\| \\
\leq & \left\|\delta_{z_{0}}\right\|\left(\left|\beta_{n}\left(f_{n}, z_{0}\right)\right|\left\|B_{z_{0}}\right\|+\beta_{n}\left(X_{z_{0}}\right)\left\|B_{z_{0}}\right\|\right) \\
\leq & K_{2}\left|\beta_{n}\left(f_{n}, z_{0}\right)\right| \\
= & K_{2} e^{u_{n}\left(z_{0}\right)}
\end{aligned}
$$

with $K_{2}=2\left\|\delta_{z_{0}}\right\|\left\|B_{z_{0}}\right\|$.

Lemma 3.2.15. Let $\left\{X_{z}: z \in \partial \mathbb{S}: X\right\}$ be an interpolation family admissible at $z_{0}$, and suppose that for a. e. $z \in \mathbb{S}_{j}$ the spaces $X_{z}$ have type $p_{j}=p_{X_{z}}$ with uniformly bounded constants, $j=0,1$. Then there is $K_{2}>0$ such that for every $n \in \mathbb{N}$,

$$
\mathbb{E}\left\|\left(\sum_{j=1}^{n} \varepsilon_{j} x_{j}\right) \log \frac{\beta_{n, 0}}{\beta_{n, 1}}-\Omega_{z_{0}}\left(\sum_{j=1}^{n} \varepsilon_{j} x_{j}\right)+\sum_{j=1}^{n} \Omega_{z_{0}}\left(\varepsilon_{j} x_{j}\right)\right\| \leq K_{2} \beta_{n, 0}^{1-\operatorname{Re}\left(z_{0}\right)} \beta_{n, 1}^{\operatorname{Re}\left(z_{0}\right)}
$$

Proof. We will use Lemma 3.2.14.

In this case, we choose $f_{n}(j+i t)=\beta_{n, j}, j=0,1$. Then we may take

$$
u_{n}(z)+i v_{n}(z)=(1-z) \log \beta_{n, 0}+z \log \beta_{n, 1}
$$

Therefore $e^{u_{n}\left(z_{0}\right)}=\beta_{n, 0}^{1-\operatorname{Re}\left(z_{0}\right)} \beta_{n, 1}^{\operatorname{Re}\left(z_{0}\right)}$ and $\beta_{n}\left(f_{n}, z\right)=\beta_{n, 0}^{1-z} \beta_{n, 1}^{z}$.

Also

$$
\beta_{n}^{\prime}\left(f_{n}, z\right)=\beta_{n}(z) \log \frac{\beta_{n, 1}}{\beta_{n, 0}}
$$

Thus, the result follows by an application of Lemma 3.2.14.

Lemma 3.2.16. Let $\left\{X_{z}: z \in \partial G ; X\right\}$ be an interpolation family admissible at $z_{0}$ and suppose that for some $n \in \mathbb{N}$ there is an absolutely log-integrable function $f_{n}: \partial G \rightarrow[0, \infty)$ such that $\beta_{n}\left(X_{z}\right) \leq f_{n}(z)$ for a. e. $z \in \partial G$. Let $v_{n}$ be a harmonic conjugate of

$$
u_{n}\left(z_{0}\right)=\int_{\partial G} \log f_{n}(z) d \mu_{z_{0}}(z)
$$

and define $\beta_{n}\left(f_{n}, z\right)=e^{u_{n}(z)+i v_{n}(z)}$.

If $\left.\Omega_{z_{0}}\right|_{W}$ is trivial, then there is $K>0$ such that

$$
\left|\frac{\beta_{n}^{\prime}\left(f_{n}, z_{0}\right)}{\beta_{n}\left(f_{n}, z_{0}\right)}\right| \beta_{n}(W) \leq K\left|\beta_{n}\left(f_{n}, z_{0}\right)\right|=K e^{u_{n}\left(z_{0}\right)}
$$

Proof. This is a direct application of Proposition 3.2.10 Lemmata 3.2.9 and 3.2.14. Indeed, let $w_{1}, \ldots, w_{n} \in B_{W}$. Then

$$
\mathbb{E}\left|\frac{\beta_{n}^{\prime}\left(f_{n}, z_{0}\right)}{\beta_{n}\left(f_{n}, z_{0}\right)}\right|\left\|\sum_{j=1}^{n} \varepsilon_{j} w_{j}\right\| \leq \mathbb{E}\left\|-\left(\sum_{j=1}^{n} \varepsilon_{j} x_{j}\right) \frac{\beta_{n}^{\prime}\left(z_{0}\right)}{\beta_{n}\left(z_{0}\right)}-\Omega_{z_{0}}\left(\sum_{j=1}^{n} \varepsilon_{j} x_{j}\right)+\sum_{j=1}^{n} \Omega_{z_{0}}\left(\varepsilon_{j} x_{j}\right)\right\|
$$




$$
\begin{aligned}
& +\mathbb{E}\left\|\Omega_{z_{0}}\left(\sum_{j=1}^{n} \varepsilon_{j} x_{j}\right)-\sum_{j=1}^{n} \Omega_{z_{0}}\left(\varepsilon_{j} x_{j}\right)\right\| \\
& \leq\left(K_{1}+K_{2}\right) e^{u_{n}\left(z_{0}\right)}
\end{aligned}
$$

Lemma 3.2.17. Let $\left\{X_{z}: z \in \partial \mathbb{S} ; X\right\}$ be an interpolation family admissible at $z_{0}$, and suppose that for a. e. $z \in \mathbb{S}_{j}$ the spaces $X_{z}$ have type $p_{j}=p_{X_{z}}$ with uniformly bounded constants, $j=0,1$. Then there is $K>0$ such that for every $n \in \mathbb{N}$

$$
\left|\log \frac{\beta_{n, 0}}{\beta_{n, 1}} \beta_{n}(W)\right| \leq K \beta_{n, 0}^{1-\operatorname{Re}\left(z_{0}\right)} \beta_{n, 1}^{\operatorname{Re}\left(z_{0}\right)}
$$

Proof. This is a particular case of Lemma 3.2.16, and follows from Proposition 3.2.13 and Lemma 3.2.15.

Proposition 3.2.18. Let $\left\{X_{z}: z \in \partial G ; X\right\}$ be an interpolation family admissible at $z_{0}$ and let $p: \partial G: \rightarrow[1,2]$ and $C: \partial G \rightarrow[1, \infty]$ be functions such that $X_{z}$ has type $p(z)$ with constant $C(z)$ for a. e. $z \in \partial G$. Suppose that

$$
\frac{1}{p\left(z_{0}\right)}=\int_{\partial G} \frac{1}{p(z)} d \mu_{z_{0}}(z)<\infty
$$

and

$$
C\left(z_{0}\right)=\exp \int_{\partial G} \log C(z) d \mu_{z_{0}}(z)<\infty
$$

Let $f_{n}(z)=C(z) n^{\frac{1}{p(z)}}$. With the notation from Lemma 3.2.16 we have

$$
\left|\beta_{n}\left(f_{n}, z_{0}\right)\right|=C\left(z_{0}\right) n^{\frac{1}{p\left(z_{0}\right)}}
$$

Proof. Notice that $\beta_{n}\left(X_{z}\right) \leq f_{n}(z)$ for a. e. $z \in \partial G$ by Lemma 3.2.7. We have

$$
\begin{aligned}
\int \log f_{n}(z) d \mu_{z_{0}}(z) & =\int_{\partial G} \log C(z) d \mu_{z_{0}}(z)+\int_{\partial G} \frac{1}{p(z)} \log n d \mu_{z_{0}}(z) \\
& =\log C\left(z_{0}\right)+\log n^{\frac{1}{p\left(z_{0}\right)}} \\
& =\log \left(C\left(z_{0}\right) n^{\frac{1}{p\left(z_{0}\right)}}\right)
\end{aligned}
$$

And therefore

$$
\left|\beta_{n}\left(f_{n}, z_{0}\right)\right|=e^{u_{n}\left(z_{0}\right)}=C\left(z_{0}\right) n^{\frac{1}{p\left(z_{0}\right)}}
$$

Theorem 3.2.19. Suppose we are in the situation of Proposition 3.2.18 and that $W$ is an infinity dimensional subspace of $X_{z_{0}}$ with $p_{W}=p\left(z_{0}\right)$ and such that $\left.\Omega_{z_{0}}\right|_{W}$ is trivial. Then $\left|\frac{\beta_{n}^{\prime}\left(f_{n}, z_{0}\right)}{\beta_{n}\left(f_{n}, z_{0}\right)}\right|$ is uniformly bounded in $n \in \mathbb{N}$.

Proof. This follows from Lemmata 3.2.7 and 3.2.16 and Proposition 3.2.18.

Corollary 3.2.20. Let $\left\{X_{z}: z \in \partial \mathbb{S} ; X\right\}$ be an interpolation family admissible at $z_{0}$, and suppose that for a. e. $z \in \mathbb{S}_{j}$ the spaces $X_{z}$ have type $p_{j}=p_{X_{z}}$ with uniformly bounded constants, $j=0,1$. Let $W$ be an infinite dimensional closed subspace of $X_{z_{0}}$ such that $p_{W}=p\left(z_{0}\right)$, where $\frac{1}{p\left(z_{0}\right)}=$ $\frac{1-\operatorname{Re}\left(z_{0}\right)}{p_{0}}+\frac{\operatorname{Re}\left(z_{0}\right)}{p_{1}}$, and such that $\Omega_{z_{0}} \mid W$ is trivial. Then $\left|\log \frac{\beta_{n, 0}}{\beta_{n, 1}}\right|$ is uniformly bounded in $n$. 
Proof. This is a particular case of Theorem 3.2.19. It may also be seen as a consequence of Lemma 3.2.17. Indeed, if the constants of the type $p_{X_{z}}$ of the spaces $X_{z}$ are uniformly bounded by $B$, we obtain by Lemma 3.2.7

$$
\beta_{n, 0}^{1-\operatorname{Re}\left(z_{0}\right)} \beta_{n, 1}^{\operatorname{Re}\left(z_{0}\right)} \leq B n^{\frac{1}{p\left(z_{0}\right)}}
$$

On the other hand, again by Lemma 3.2.7, we have $n^{\frac{1}{p\left(z_{0}\right)}} \leq \beta_{n}(W)$, and the result follows.

Theorem 3.2.21. Let $\left\{X_{z}: z \in \partial \mathbb{S} ; X\right\}$ be an interpolation family admissible at $z_{0}$, and suppose that for a. e. $z \in \mathbb{S}_{j}$ the spaces $X_{z}$ have type $p_{j}=p_{X_{z}}$ with uniformly bounded constants, $j=0,1$, and suppose $p_{0} \neq p_{1}$. Let $p\left(z_{0}\right)$ be given by $\frac{1}{p\left(z_{0}\right)}=\frac{1-\operatorname{Re}\left(z_{0}\right)}{p_{0}}+\frac{\operatorname{Re}\left(z_{0}\right)}{p_{1}}$.

1. If $W$ if an infinite dimensional subspace of $X_{z_{0}}$ such that $p_{W}=p\left(z_{0}\right)$, then $\left.\Omega_{z_{0}}\right|_{W}$ is not trivial.

2. If $X_{z_{0}}$ is infinite dimensional and $p_{W}=p\left(z_{0}\right)$ for every infinite dimensional closed subspace $W$ of $X_{z_{0}}$, then $\Omega_{z_{0}}$ is singular.

Proof. By hypothesis and Lemma 3.2.7, there is a constant $C>0$ such that $n^{\frac{1}{p_{j}}} \leq \beta_{n, j} \leq C n^{\frac{1}{p_{j}}}$, $j=0,1$. Therefore

$$
C^{-1} n^{\frac{1}{p_{0}}-\frac{1}{p_{1}}} \leq \log \frac{\beta_{n, 0}}{\beta_{n, 1}} \leq C n^{\frac{1}{p_{0}}-\frac{1}{p_{1}}}
$$

and therefore $\left|\log \frac{\beta_{n, 0}}{\beta_{n, 1}}\right|$ cannot be uniformly bounded.

If $\left.\Omega_{z_{0}}\right|_{W}$ was trivial, then by Corollary 3.2.20 we would have $\left|\log \frac{\beta_{n, 0}}{\beta_{n, 1}}\right|$ uniformly bounded in $n$. Therefore, $\left.\Omega_{z_{0}}\right|_{W}$ is not trivial.

2. follows directly from 1 .

\subsection{Duality results}

While there is a clear interpolation result for the type of the spaces in the interpolation scale (Theorem 3.1.10), no such result is possible for cotype (GARLING; MONTGOMERYSMITH, 1991). Garling and Montgomery-Smith prove this result the following way: they show that for any separable Banach space $X$ there is a compatible couple $\left(X_{0}, X_{1}\right)$ such that $X_{0}$ and $X_{1}$ are isomorphic to $\ell_{1}$ and for any $\theta \in(0,1)$ the space $X_{\theta}$ contains a complemented copy of $X$.

So, if we take as $X$ the space $c_{0}$, which best cotype is $\infty$, we see that having nontrivial cotype does not pass to the interpolated space.

However, in this example the spaces being interpolated have best type 1, contradicting the hypothesis of Theorem 3.1.6 on the duality between type and cotype. So we may ask if we have an interpolating result regarding cotype if we suppose that the spaces being interpolated have nontrivial type. The answer is yes, under additional hypotheses that tell us what happens when we interpolate dual spaces.

Theorem 3.3.1 ((CALDERÓN, 1964)). Let $\bar{X}=\left(X_{0}, X_{1}\right)$ be a compatible couple of Banach spaces such that $\Delta(\bar{X})=X_{0} \cap X_{1}$ is dense in $X_{0}$ and in $X_{1}$ and that at least one of the spaces, $X_{0}$ or $X_{1}$, is reflexive. Then $\left(X_{0}^{*}, X_{1}^{*}\right)$ is a compatible couple and for every $\theta \in(0,1)$ we have $\left(X_{0}^{*}, X_{1}^{*}\right)_{\theta}=\left(X_{0}, X_{1}\right)_{\theta}^{*}$ isometrically.

Proposition 3.3.2. Let $\left(X_{0}, X_{1}\right)$ be a compatible couple, and suppose that $X_{j}$ has cotype $q_{j}$, $j=0,1$. Under the hypotheses of Theorem 3.3.1, and supposing that $X_{0}$ and $X_{1}$ have nontrivial type, the space $X_{\theta}$ has cotype $q_{\theta}$ given by $\frac{1}{q_{\theta}}=\frac{1-\theta}{q_{0}}+\frac{\theta}{q_{1}}$, for $\theta \in(0,1)$. 
Proof. For $j=0,1$, let $p_{j}$ be given by $\frac{1}{p_{j}}+\frac{1}{q_{j}}=1$. By Theorem 3.1.6, $X_{j}^{*}$ has type $p_{j}, j=0,1$, and by Theorem 3.1.10 $\left(X_{0}^{*}, X_{1}^{*}\right)_{\theta}$ has type $p_{\theta}$ given by $\frac{1}{p_{\theta}}=\frac{1-\theta}{p_{0}}+\frac{\theta}{p_{1}}$, for $\theta \in(0,1)$. But:

Again by Theorem 3.1.6 and by Theorem 3.3.1, $X_{\theta}$ has cotype $q$ given by $\frac{1}{p_{\theta}}+\frac{1}{q}=1$.

$$
\begin{aligned}
\frac{1}{q} & =1-\frac{1}{p_{\theta}} \\
& =1-\frac{1-\theta}{p_{0}}-\frac{\theta}{p_{1}} \\
& =1-(1-\theta)\left(1-\frac{1}{q_{0}}\right)-\theta\left(1-\frac{1}{q_{1}}\right) \\
& =\frac{1-\theta}{q_{0}}+\frac{\theta}{q_{1}} \\
& =\frac{1}{q_{\theta}}
\end{aligned}
$$

Therefore, $X_{\theta}$ has cotype $q_{\theta}$.

To obtain Theorem 3.2.21 we used several times the triangle inequality. Being an upper estimate, cotype does not seem fit to this kind of approach. One solution is to use Theorem 3.3.1 and work with the type of the dual space, as in Proposition 3.3.2. In order to use this approach to obtain a result regarding twisted sums, we also need to know how the twisting behaves under duality.

Notation 3.3.3. If $\bar{X}=\left(X_{0}, X_{1}\right)$ is a compatible couple of Banach spaces, we will denote $\overline{X^{*}}=$ $\left(X_{0}^{*}, X_{1}^{*}\right)$.

Under the hypotheses of Theorem 3.3.1, we will denote $X_{\theta}^{*}=\left(X_{0}^{*}, X_{1}^{*}\right)_{\theta}=\left(X_{0}, X_{1}\right)_{\theta}^{*}$.

Observation 3.3.4. The identification in Theorem 3.3.1 is an isometric identification which we treat like an equality. Accordingly, we will speak of equivalence of twisted sums in which $X_{\theta}^{*}$ appears when strictly speaking we have an isomorphic equivalence.

From now until the end of this section we assume we are under the assumptions of Theorem 3.3.1.

If we dualize the twisted sum

$$
0 \longrightarrow X_{\theta} \longrightarrow d X_{\theta} \longrightarrow X_{\theta} \longrightarrow 0
$$

we obtain another twisted sum

$$
0 \longrightarrow X_{\theta}^{*} \longrightarrow\left(d X_{\theta}\right)^{*} \longrightarrow X_{\theta}^{*} \longrightarrow 0
$$

On the other hand, the interpolation scale $\left(X_{0}^{*}, X_{1}^{*}\right)_{\theta}$ induces a twisted sum

$$
0 \longrightarrow X_{\theta}^{*} \longrightarrow d\left(X_{\theta}^{*}\right) \longrightarrow X_{\theta}^{*} \longrightarrow 0
$$

The twisted sums 3.6 and 3.7 are equivalent ((ROCHBERG; WEISS, 1983) and (CWIKEL et al., 1989)). We prove this fact here to motivate the proof in the operator space setting later. We will need several prior results. 
Proposition 3.3.5 ((CALDERÓN, 1964)). Let $\bar{X}=\left(X_{0}, X_{1}\right)$ be a compatible couple of Banach spaces such that $\Delta(\bar{X})$ is dense in $X_{0}$ and in $X_{1}$. Then $\Delta(\bar{X})^{*}=\Sigma\left(\overline{X^{*}}\right)$ and $\Sigma(\bar{X})^{*}=\Delta\left(\overline{X^{*}}\right)$ isometrically. Furthermore, under the assumptions of Theorem 3.3.1, these identifications are compatible with the identification $\left(X_{\theta}\right)^{*}=\left(X_{0}^{*}, X_{1}^{*}\right)_{\theta}$.

In the same way that $\Delta(\bar{X})$ is dense in $X_{\theta}$, we have that $\Delta(\bar{X}) \times \Delta(\bar{X})$ is dense in $d X_{\theta}$.

Lemma 3.3.6. Let $\bar{X}$ be a compatible couple. Then $\Delta(\bar{X}) \times \Delta(\bar{X})$ is dense in $d X_{\theta}$. Furthermore, we may use functions in $\mathscr{F}_{0}$ to calculate the norm of elements in $\Delta(\bar{X}) \times \Delta(\bar{X}) \subset d X_{\theta}$, when we view it as a quotient of $\mathscr{F}$.

Proof. The first part follows from $\mathscr{F}_{0}$ being dense in $\mathscr{F}$ (Proposition 2.3.11).

The second is an adaptation of the already referenced Stafney's argument of ((STAFNEY, 1969), Lemma 2.5): let $(x, y) \in \Delta(\bar{X})^{2}$, and $f \in \mathscr{F}_{0}$ be such that $f(\theta)=x, f^{\prime}(\theta)=y$. We have

$$
\|(x, y)\|=\inf \left\{\|f+g\|: g \in \mathscr{F}, g(\theta)=g^{\prime}(\theta)=0\right\}
$$

Let $g \in \mathscr{F}$ with $g(\theta)=g^{\prime}(\boldsymbol{\theta})=0$, and let $\varphi: \mathbb{S} \rightarrow \mathbb{D}$ be a conformal map with $\varphi(\theta)=0$. Then $\frac{g}{\varphi^{2}} \in \mathscr{F}$, and up to any error we may approximate it by a function $h_{1} \in \mathscr{F}_{0}$; consequently $h=\varphi^{2} h_{1} \in \mathscr{F}_{0}$ approximates $g$.

This implies that

$$
\|(x, y)\|=\inf \left\{\|f+h\|: h \in \mathscr{F}_{0}, h(\theta)=h^{\prime}(\theta)=0\right\}
$$

The following result basically says that compatible couples originate admissible families.

Lemma 3.3.7 ((STAFNEY, 1969)). Let $\bar{X}$ be a compatible couple and $x \in \Delta(\bar{X})$. Then

$$
\|x\|_{\theta}=\inf \left\{\|f\|: f \in \mathscr{F}_{0}(\bar{X}), f(\theta)=x\right\}
$$

for every $\theta \in(0,1)$.

Corollary 3.3.8. We may take a section $B_{\theta}$ for $\delta_{\theta}$ such that $B_{\theta}(x) \in \mathscr{F}_{0}(\bar{X})$ for every $x \in \Delta(\bar{X})$.

We will denote by $\delta_{\theta}$ and by $\delta_{\theta}^{\prime}$ the maps of evaluation at $\theta$ and evaluation of the derivative at $\theta$, respectively, both on $\mathscr{F}(\bar{X})$ and on $\mathscr{F}\left(\overline{X^{*}}\right)$.

Let $B_{\theta}$ and $B_{\theta}^{*}$ be sections for $\delta_{\theta}$ on $X_{\theta}$ and on $X_{\theta}^{*}$, respectively, and suppose that they satisfy the condition of Corollary 3.3.8. We will denote the corresponding $\Omega$-maps by $\Omega_{\theta}$ and $\Omega_{\theta}^{*}$, respectively.

Lemma 3.3.9 (Schwarz-Pick, (KRANTZ, 1999)). Let $f: \mathbb{D} \rightarrow \mathbb{D}$ be analytic. Then:

$$
\left|f^{\prime}(a)\right| \leq \frac{1-|f(a)|^{2}}{1-|a|^{2}}
$$

for every $a \in \mathbb{D}$.

Lemma 3.3.10 ((ROCHBERG; WEISS, 1983)). There is a constant $c>0$ such that for every $y \in \Delta(\bar{X})$ and every $y^{*} \in \Delta\left(\overline{X^{*}}\right)$ we have:

$$
\left|<\Omega_{\theta}^{*} y^{*}, y>+<y^{*}, \Omega_{\theta} y>\right| \leq c\left\|y^{*}\right\|_{X_{\theta}^{*}}\|y\|_{X_{\theta}}
$$


Observation 3.3.11. Formula 3.8 is well-defined because of Proposition 3.3.5.

Proof. Fix $y \in \Delta(\bar{X})$ and $y^{*} \in \Delta\left(\overline{X^{*}}\right)$, and let $H: \mathbb{S} \rightarrow \mathbb{C}$ be given by

$$
H(z)=\left\langle B_{\theta}^{*} y^{*}(z), B_{\theta} y(z)\right\rangle
$$

By our choice of $B_{\theta}$ and $B_{\theta}^{*}$, we have that $H$ is analytic and

$$
H^{\prime}(z)=\left\langle\left(B_{\theta}^{*} y^{*}\right)^{\prime}(z), B_{\theta} y(z)\right\rangle+\left\langle B_{\theta}^{*} y^{*}(z),\left(B_{\theta} y\right)^{\prime}(z)\right\rangle
$$

so that

$$
H^{\prime}(\theta)=\left\langle\Omega_{\theta}^{*} y^{*}, y\right\rangle+\left\langle y^{*}, \Omega_{\theta} y\right\rangle
$$

By Theorem 3.3.1, for every $z \in \mathbb{S}$ we have

$$
\begin{aligned}
|H(z)| & \leq\left\|B_{\theta}^{*} y^{*}(z)\right\| X_{X_{z}^{*}}\left\|B_{\theta} y(z)\right\|_{X_{z}} \\
& \leq\left\|B_{\theta}^{*} y^{*}\right\|\left\|B_{\theta} y\right\| \\
& \leq\left\|B_{\theta}^{*}\right\|\left\|B_{\theta}\right\|\left\|y^{*}\right\|_{X_{\theta}^{*}}\|y\|_{X_{\theta}}
\end{aligned}
$$

Let $\varphi: \mathbb{D} \rightarrow \mathbb{S}$ be a conformal map with $\varphi(0)=\theta$, and let $G=\frac{H \circ \varphi}{2\left\|B_{\theta}^{*}\right\|\left\|B_{\theta}\right\|\left\|y^{*}\right\|_{X_{\theta}^{*}}^{*}\|y\|_{X_{\theta}}}$ : $\mathbb{D} \rightarrow \mathbb{D}$. By Schwarz-Pick Lemma 3.3.9, we have

$$
\left|G^{\prime}(0)\right| \leq 1
$$

But

$$
G^{\prime}(0)=\frac{H^{\prime}(\theta) \varphi^{\prime}(0)}{2\left\|B_{\theta}^{*}\right\|\left\|B_{\theta}\right\|\left\|y^{*}\right\| X_{\theta}^{*}\|y\|_{X_{\theta}}}
$$

and since $y$ and $y^{*}$ were arbitrary we obtain the result with $c=\frac{2\left\|B_{\theta}^{*}\right\|\left\|B_{\theta}\right\|}{\varphi^{\prime}(0)}$.

Theorem 3.3.12 ((ROCHBERG; WEISS, 1983)). The extensions

$$
0 \longrightarrow X_{\theta}^{*} \longrightarrow\left(d X_{\theta}\right)^{*} \longrightarrow X_{\theta}^{*} \longrightarrow 0
$$

and

$$
0 \longrightarrow X_{\theta}^{*} \longrightarrow d\left(X_{\theta}^{*}\right) \longrightarrow X_{\theta}^{*} \longrightarrow 0
$$

are equivalent.

Proof. We will show that there is an operator $T: d\left(X_{\theta}^{*}\right) \rightarrow\left(d X_{\theta}\right)^{*}$ making the following diagram commute

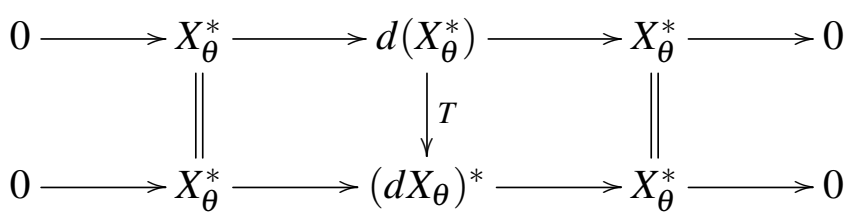

By Lemma 3.3.6, the subspace

$$
A=\left\{\left(x^{*}, y^{*}\right) \in d\left(X_{\theta}^{*}\right): x^{*}, y^{*} \in \Delta\left(\overline{X^{*}}\right)\right\}
$$

is dense in $d\left(X_{\theta}^{*}\right)$, and the subspace

$$
B=\left\{(x, y) \in d X_{\theta}: x, y \in \Delta(\bar{X})\right\}
$$


is dense in $d X_{\theta}$. For $\left(x^{*}, y^{*}\right) \in A$ and $(x, y) \in B$, let

$$
T\left(x^{*}, y^{*}\right)(x, y)=\left\langle x^{*}, y\right\rangle+\left\langle y^{*}, x\right\rangle
$$

By Lemma 3.3.10, we have for some constant $c$ independent of $\left(x^{*}, y^{*}\right)$ and $(x, y)$,

$$
\begin{aligned}
\left|T\left(x^{*}, y^{*}\right)(x, y)\right|= & \left|\left\langle x^{*}, y\right\rangle+\left\langle y^{*}, x\right\rangle\right| \\
= & \left|\left\langle x^{*}-\Omega_{\theta}^{*} y^{*}, y\right\rangle+\left\langle\Omega_{\theta}^{*} y^{*}, y\right\rangle+\left\langle y^{*}, \Omega_{\theta} y\right\rangle+\left\langle y^{*}, x-\Omega_{\theta} y\right\rangle\right| \\
\leq & \left\|x^{*}-\Omega_{\theta}^{*} y^{*}\right\|_{X_{\theta}^{*}}\|x\|_{\theta}+\left|\left\langle\Omega_{\theta}^{*} y^{*}, y\right\rangle+\left\langle y^{*}, \Omega_{\theta} y\right\rangle\right| \\
& +\left\|y^{*}\right\|_{X_{\theta}^{*}}\left\|x-\Omega_{\theta} y\right\|_{X_{\theta}} \\
\leq & (2+c)\left\|\left(x^{*}, y^{*}\right)\right\|\|(x, y)\|
\end{aligned}
$$

Therefore, $T\left(x^{*}, y^{*}\right)$ extends to an element of $\left(d X_{\theta}\right)^{*}$ of norm at most $(2+c)\left\|\left(x^{*}, y^{*}\right)\right\|$.

In turn, $T$ is a linear map defined from $A$ into $\left(d X_{\theta}\right)^{*}$ with norm at most $2+c$, and therefore extends to all of $d\left(X_{\theta}^{*}\right)$. Then $T$ makes the diagram commute, and by the 3-lemma 2.2.17, we obtain the theorem.

Finally, we are able to obtain:

Theorem 3.3.13. Let $\bar{X}=\left(X_{0}, X_{1}\right)$ be a compatible couple of Banach spaces such that $\Delta(\bar{X})$ is dense in $X_{0}$ and $X_{1}$ and at least one of the spaces $X_{0}, X_{1}$ is reflexive. Suppose also that $X_{j}$ has nontrivial type and has cotype $q_{X_{j}}, j=0,1$, and that $q_{X_{0}} \neq q_{X_{1}}$. If $X_{\theta}$ is infinite dimensional and $q_{X_{\theta}}$ satisfies $\frac{1}{q_{X_{\theta}}}=\frac{1-\theta}{q_{X_{0}}}+\frac{\theta}{q_{X_{1}}}$, then $\Omega_{\theta}$ is nontrivial.

Proof. By the hypotheses and Theorem 3.1.6, we have that $X_{j}^{*}$ has type $p_{X_{j}^{*}}, j=0,1$, and $p_{X_{0}^{*}} \neq p_{X_{1}^{*}}$. By interpolation, $X_{\theta}$ also has nontrivial type, and by another application of Theorem

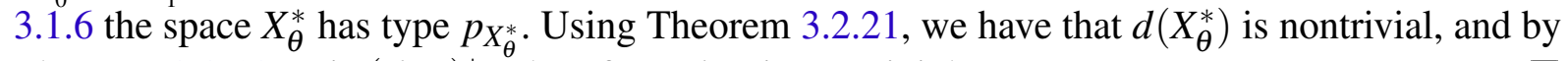
Theorem 3.3.12 so is $\left(d X_{\theta}\right)^{*}$. Therefore, $d X_{\theta}$ is nontrivial.

\subsection{Examples and applications}

In this section we present some known examples and comment on what they say about the results we obtained so far. In particular, we show that Theorem 3.2.21 is optimal. Then, we apply our results to the study of the Schatten classes, and obtain nontrivial examples of twisted sums of spaces without the compact approximation property.

\subsection{1 $\ell_{p}$ spaces}

We begin with the classical scale of $\ell_{p}$ spaces: $\left(\ell_{p_{0}}, \ell_{p_{1}}\right)_{\theta}=\ell_{p}, \frac{1}{p}=\frac{1-\theta}{p_{0}}+\frac{\theta}{p_{1}}$, for $p_{0}, p_{1} \in[1, \infty], \theta \in(0,1)$.

From the Reiteration Theorem 2.3.18, if we fix $p$ the derived short exact sequence $\ell_{p} \rightarrow d \ell_{p} \rightarrow \ell_{p}$ does not depend on the choice of $p_{0}$ and $p_{1}$ (up to projective equivalence). Recall that $d \ell_{p}$ is the Kalton-Peck spaces $Z_{p}$.

Recall from Examples 3.1.12 and 3.1.13 that $p_{\ell_{p^{\prime}}}=\min \left\{p^{\prime}, 2\right\}$ and $q_{\ell_{p^{\prime}}}=\max \left\{2, p^{\prime}\right\}$, $p^{\prime} \in[1, \infty)$, and that $p_{\ell_{\infty}}=1, q_{\ell_{\infty}}=\infty$.

We start by applying Theorem 3.2.21: consider the interpolation scale $\left(\ell_{2}, \ell_{1}\right)$. Then for every $\theta \in(0,1)$ we have that $\left(\ell_{2}, \ell_{1}\right)_{\theta}=\ell_{p^{\prime}}, \frac{1}{p^{\prime}}=\frac{1-\theta}{2}+\theta$. 
Since $\frac{1}{p_{\ell_{p^{\prime}}}}=\frac{1}{p^{\prime}}$, and $\ell_{p^{\prime}}$ is $\ell_{p^{\prime}}$-saturated, we have that the conditions of Theorem 3.2.21 are satisfied, and consequently $Z_{p^{\prime}}$ is a singular extension of $\ell_{p^{\prime}}$, for $p^{\prime} \in(1,2)$.

To apply Theorem 3.3.13, let $2<q<\infty$ and consider the interpolation scale $\left(\ell_{q}, \ell_{2}\right)$. Then $\left(\ell_{q}, \ell_{2}\right)_{\theta}=\ell_{q^{\prime}}, \frac{1}{q^{\prime}}=\frac{1-\theta}{q}+\frac{\theta}{2}$.

Since $\frac{1}{q_{q_{q^{\prime}}}}=\frac{1}{q^{\prime}}$, we have that the $Z_{q^{\prime}}$ is a nontrivial extension of $\ell_{q^{\prime}}$. Since we may choose $q$ at will, we have that $Z_{q^{\prime}}$ is nontrivial for every $2<q^{\prime}<\infty$.

As we mentioned before, it is known that the short exact sequence induced by interpolation $\ell_{p} \rightarrow Z_{p} \rightarrow \ell_{p}$ is singular for every $p \in(1, \infty)$. However, using Theorems 3.2.21 and 3.3.13 we are not able to retrieve this information. The reason is that type is a relatively weak concept in comparison with all the structure that the $\ell_{p}$ spaces have. The usefulness of Theorems 3.2.21 and 3.3.13 is explored in Sections 3.4.4 and 3.4.5: obtaining structural results once we know a twisted sum is not singular, and obtaining nontriviality results when considering scales of spaces without a rich structure (no basis or function space structure).

\subsection{2 $L_{p}$ spaces}

Consider the $L_{p}$ spaces on $[0,1]$. As in the previous section, we have $\left(L_{p_{0}}, L_{p_{1}}\right)_{\theta}=L_{p}$, $\frac{1}{p}=\frac{1-\theta}{p_{0}}+\frac{\theta}{p_{1}}$, for every $p_{0}, p_{1} \in[1, \infty], \theta \in(0,1)$. By the Reiteration Theorem 2.3.18, for each $p \in(1, \infty)$ we have unambiguously defined an extension

$$
0 \longrightarrow L_{p} \longrightarrow Z L_{p} \longrightarrow L_{p} \longrightarrow 0
$$

up to projective equivalence.

As before, using Theorems 3.2.21 and 3.3.13 we have that (3.9) is nontrivial for every $p \in(1, \infty) \backslash\{2\}$. However, for $1<p<2$ it is not singular ((SUÁREZ DE LA FUENTE, 2013), see also (CABELLO SÁNCHEZ, 2014)). More specifically, we have:

Theorem 3.4.1 ((SUÁREZ DE LA FUENTE, 2013), Proposition 4.1). If $1<p<q \leq 2$, then there is a copy of $\ell_{q}$ inside $L_{p}$ such that if $\Omega$ is the quasilinear map induced by the interpolation scale $\left(L_{\infty}, L_{1}\right)$, then $\left.\Omega\right|_{\ell_{q}}$ is trivial.

This shows that Theorem 3.2.21 is optimal.

\subsubsection{Ferenczi's spaces revisited}

In Section 2.4 we showed that Ferenczi's spaces from (FERENCZI, 1997) may be defined through our method of interpolation. Originally these spaces are also defined by means of complex interpolation, but through another method. In (CASTILLO; FERENCZI; GONZÁLEZ, 2017) it is shown that the twisted sum of Ferenczi's spaces induced by complex interpolation are singular. We show how our methods may be used to obtain these results in some cases (not all, as for the interpolation couple $\left.\left(\ell_{\infty}, \ell_{1}\right)\right)$.

Fix $\theta \in(0,1)$ and $1<p<\infty$. The Ferenczi's space $\mathscr{F}_{p}$ is obtained by interpolating a family $\left\{X_{z}\right\}_{z \in \partial \mathbb{S}}$, where $X_{i t}$ are Gowers-Maurey type spaces, and $X_{1+i t}=\ell_{p}, t \in \mathbb{R}$.

Consider the function $f(x)=\log _{2}(x+1)$. According to (FERENCZI, 1997), we have that if $x_{1}, \ldots, x_{n}$ are successive vectors in $X_{i t}$, then

$$
\frac{1}{f(n)} \sum_{j=1}^{n}\left\|x_{j}\right\|_{i t} \leq\left\|\sum_{j=1}^{n} x_{j}\right\|_{i t}
$$


If the space $X_{i t}$ had type $p>1$ then for some constant $C>0$ we would have (take $x_{1}, \ldots, x_{n}$ all of norm 1$)$ :

$$
n \leq C \log _{2}(n+1) n^{\frac{1}{p}}
$$

which would imply

$$
2^{n-n^{\frac{1}{p}}} \leq 2^{C}(n+1)
$$

which is a contradiction. Therefore, $p_{X_{i t}}=1$, for all $t \in \mathbb{R}$. Consider the following results from (CASTILLO; FERENCZI; MORENO, 2014):

Proposition 3.4.2 ((CASTILLO; FERENCZI; MORENO, 2014), Proposition 5.4). Let $n \in \mathbb{N}$ and $\varepsilon>0$. Then, there exist vectors $u_{1}, \ldots, u_{n} \in c_{00}$ such that $1 \leq\left\|u_{j}\right\|_{t} \leq 1+\varepsilon, j=1, \ldots, n$, and for any $a_{1}, \ldots, a_{n} \in \mathbb{C}$ of modulus 1 and every $t \in \mathbb{S}_{0}^{\infty}$, we have $\left\|a_{1} u_{1}+\ldots+a_{n} u_{n}\right\|_{i t} \leq 1+\varepsilon$, where $\mathbb{S}_{0}^{\infty}$ is a subset of $\mathbb{S}_{0}$ of total measure.

This readily implies that $q_{X_{i t}}=\infty$ for a set of total measure.

Proposition 3.4.3 ((CASTILLO; FERENCZI; MORENO, 2014), Proposition 4.4). Let $1<p<$ $\infty$.

1. If $p>2$, then $\mathscr{F}_{p}$ has cotype $\frac{p}{\theta}=q_{\mathscr{F}_{p}}$.

2. If $p \leq 2$, then $\mathscr{F}_{p}$ has type $1-\theta+\frac{\theta}{p}=p_{\mathscr{F}_{p}}$.

Finally, we have the following result from (CASTILLO; FERENCZI; GONZÁLEZ, 2017):

Proposition 3.4.4 ((CASTILLO; FERENCZI; GONZÁLEZ, 2017), Proposition 8.4). Let $Y$ be a block subspace of $\mathscr{F}_{p}, n \in \mathbb{N}$, and $\varepsilon>0$. Then there exists a block subsequence $y_{1}<\ldots<y_{n}$ in $Y$ with $1 \leq\left\|y_{j}\right\| \leq 1+\varepsilon, j=1, \ldots, n$, such that for any complex $a_{1}, \ldots, a_{n}$, we have $\left\|\sum a_{j} y_{j}\right\|_{\mathscr{F}_{p}} \geq$ $(1+\varepsilon)^{-1}\left(\sum\left|a_{j}\right|^{p^{\prime}}\right)^{\frac{1}{p^{\prime}}}$, where $\frac{1}{p^{\prime}}=1-\theta+\frac{\theta}{p}$.

This implies that for $1<p<2$ and for every block subspace $Y$ of $\mathscr{F}, p_{Y}=p^{\prime}=p_{\mathscr{F}}$. Applying Theorem 3.2.21 we see that the quasi-linear map induced by complex interpolation is nontrivial on every block subspace of $\mathscr{F}_{p}$, which is enough to prove that it is singular ((CASTILLO; MORENO, 2002; SÁNCHEZ; CASTILLO; SUÁREZ, 2012)).

For values of $p$ bigger than 2 , notice that, for $t \in \mathbb{S}_{0}^{\infty}$, if $X_{i t}^{*}$ had type bigger than $1, X_{i t}^{* *}$ would have finite cotype, and that would imply that $X_{i t}$ has finite cotype. By considering the dual interpolation scale, and using Theorem 3.2.21, we see that the dual extension is nontrivial, and therefore the original extension is nontrivial.

\subsubsection{Schatten classes}

We begin this section by recalling the definition of the Schatten classes $S_{p}$.

For $p=\infty, S_{\infty}=\mathscr{K}\left(\ell_{2}\right)$, the space of compact operators on $\ell_{2}$.

For $p \in[1, \infty)$ the Schatten class $S_{p}$ is the Banach space of compact operators $T \in B\left(\ell_{2}\right)$ for which $\|T\|_{p}^{p}=\operatorname{trace}\left(\left(x^{*} x\right)^{\frac{p}{2}}\right)<\infty$. Equivalently, it is the class of compact operators $T$ for which the singular values $\sigma_{n}(T)$ form a sequence in $\ell_{p}$, equipped with the norm $\|T\|_{p}=$ $\left\|\left(\sigma_{n}(T)\right)_{n}\right\|_{\ell_{p}}$.

The Schatten classes $S_{p}$ are $B(H)$-bimodules with the operation of composition of operators. 
As for the $\ell_{p}$ spaces, we have that $p_{S_{p^{\prime}}}=\min \left\{p^{\prime}, 2\right\}$ and $q_{S_{p^{\prime}}}=\max \left\{2, p^{\prime}\right\}, p^{\prime} \in[1, \infty)$. Also, for $p_{0}, p_{1} \in[1, \infty]$ we have the isometry $\left(S_{p_{0}}, S_{p_{1}}\right)=S_{p}, \frac{1}{p}=\frac{1-\theta}{p_{0}}+\frac{\theta}{p_{1}}$.

Therefore, for $1<p<\infty$ there is an extension $\Phi_{p}$ of $S_{p}$ induced by interpolation. Let $\Omega_{p}$ denote the corresponding quasilinear map. We have:

Theorem 3.4.5 ((SUÁREZ DE LA FUENTE, 2014)). Let $p \in(1, \infty)$ and let $V$ be a closed (left or right) ideal of $S_{p}$. Then the following are equivalent:

1. $\left.\Omega_{p}\right|_{V}$ is nontrivial.

2. $\max \{r k(T): T \in V\}=\infty$, where $r k(T)$ is the rank of $T$.

Notice that the only bilateral ideal of $S_{p}$ is $S_{p}$ itself.

Using this, Theorem 3.2.21 and classical facts about the Schatten classes, we are able to obtain structural results on the submodules of $S_{p}$, for $1<p<2$. We will use the following result:

Theorem 3.4.6 ((ARAZY; LINDENSTRAUSS, 1975)). Let $p \in[1, \infty)$ and $\left(x_{n}\right)$ be a normalized basic sequence in $S_{p}$. Then there is a subsequence $\left(x_{n_{k}}\right)$ of $\left(x_{n}\right)$ which is equivalent to the canonical basis of $\ell_{2}$ or of $\ell_{p}$ and such that its span is complemented in $S_{p}$.

Theorem 3.4.7. Let $1<p<2$ and let $V$ be a closed (left or right) ideal of $S_{p}$ for which $\max \{r k(T): T \in V\}<\infty$. Then:

1. $V$ does not contain an isomorphic copy of $\ell_{p}$.

2. $V$ does not contain an isomorphic copy of $S_{p}$.

3. Every basic sequence in $V$ admits a subsequence equivalent to the canonical basis of $\ell_{2}$ such that its span is complemented in $S_{p}$.

Proof. Consider the interpolation scale $\left(S_{2}, S_{1}\right)$. We have for $1<p<2$ that $S_{p}=\left(S_{2}, S_{1}\right)_{\theta}$, $\frac{1}{p}=\frac{1-\theta}{2}+\theta$. Therefore, the conditions on $p_{S_{1}}, p_{S_{2}}$ and $p_{S_{p}}$ of Theorem 3.2.21 are satisfied. Let $\Psi_{p}$ denoted the quasilinear map induced by the interpolation scale $\left(S_{2}, S_{1}\right)$ at $p$ (which is projectively equivalent to $\Omega_{p}$ ).

We have by Theorem 3.4.5 that $\left.\Psi_{p}\right|_{V}$ is trivial. Therefore, by 3.2.21, we have that $p_{V}>p_{S_{p}}$. This implies 1., since $p_{\ell_{p}}=p_{S_{p}}$, and 2.. In turn, 3. follows from 1 . and Theorem 3.4.6.

We do not know if Theorem 3.4.7 may be obtained from the results of (CASTILLO; FERENCZI; GONZÁLEZ, 2017).

\subsubsection{Spaces without the approximation property}

In this section we present twisted sums obtained through interpolation of spaces without the compact approximation property (CAP). Let us recall the classical definition.

Definition 3.4.8. A Banach space $X$ is said to have the compact approximation property $(C A P$, for short) iffor every compact set $K \subset X$ and every $\varepsilon>0$ there is a compact operator $T: X \rightarrow X$ such that

$$
\|T x-x\|<\varepsilon
$$

for every $x \in K$.

Examples of spaces with the CAP are Banach spaces with a basis.

We will interpolate the spaces of Szankowski (SZANKOWSKI, 1978), which are subspaces of the $\ell_{p}$ spaces without the CAP. 
We point out that complex interpolation of spaces which are not Köthe function spaces (spaces with a 1-unconditional basis are Köthe function spaces) in general is not simple. While for a couple of Köthe function spaces $\left(X_{0}, X_{1}\right)$ we have the interpolation formula

$$
X_{\theta}=X_{0}^{\theta} X_{1}^{1-\theta}
$$

for general couples there is not an easy way to calculate the interpolation space. But, even without an explicit description of the interpolation and derived spaces, we are able to obtain interesting properties of our examples.

We will use the presentation of the spaces of Szankowski from (LINDENSTRAUSS; TZAFRIRI, 1996). The following criterion for failure of the CAP is Proposition II.1.g.1 of (LINDENSTRAUSS; TZAFRIRI, 1996), and the comment thereafter.

Proposition 3.4.9. Let $X$ be a Banach space. If the following condition holds, then $X$ does not have the CAP: there is a bounded sequence $\left(x_{n}\right) \subset X, a w^{*}$-null sequence $\left(x_{n}^{*}\right) \subset X^{*}$ and sequence of finite subsets $F_{n} \subset X$ such that:

1. $x_{n}^{*}\left(x_{n}\right)=1$ for every $n \in \mathbb{N}$;

2. $\sum_{n=1}^{\infty} \sup \left\{\|x\|: x \in F_{n}\right\}<\infty$;

3. $\left|\alpha_{n}(T)-\alpha_{n-1}(T)\right| \leq \sup \left\{\|T x\|: x \in F_{n}\right\}$ for every $n \in \mathbb{N}, T \in B(X)$, where $\beta_{0} \equiv 0$ and for $n \geq 1$ :

$$
\alpha_{n}(T)=2^{-n} \sum_{j=2^{n}+1}^{2^{n+1}} x_{j}^{*}\left(T x_{j}\right)
$$

Notation 3.4.10. 1. $\sigma_{n}=\left\{2^{n}, 2^{n}+1, \ldots, 2^{n+1}-1\right\} \subset \mathbb{N}$

For every $i \geq 2$ we let

2. $f_{k}(4 i+l)=2 i+k-l, l=0,1,2,3, k=1,2$

3. $f_{k}(4 i+l)=4 i+((l+k-2) \bmod 4), l=0,1,2,3, k=3,4,5$

4. $f_{k}(4 i+l)=8 i+k-6, l=0,1, k=6,7,8,9$

5. $f_{k}(4 i+l)=8 i+k-2, l=2,3, k=6,7,8,9$

Lemma 3.4.11 ((LINDENSTRAUSS; TZAFRIRI, 1996), Lemma II.1.g.5). For every $n \geq 2$ there are partitions $\Delta_{n}$ and $\nabla_{n}$ of $\sigma_{n}$ and a sequence of integers $\left(m_{n}\right)$ such that, for every $n \geq 2$,:

1. $m_{n} \geq 2^{\frac{n}{8}-2}$;

2. For every $A \in \nabla_{n}$ we have $m_{n} \leq \# A \leq 2 m_{n}$;

3. For every $A \in \nabla_{n}$ and for every $B \in \Delta_{n}$ we have $\#(A \cap B) \leq 1$;

4. For every $A \in \nabla_{n}, n \geq 3$, and every $1 \leq k \leq 9$, there is $B \in \Delta_{n-1}, \Delta_{n}$ or $\Delta_{n+1}$ such that $f_{k}(A) \subset B$.

In this section we will denote by $X_{p}$ the following space $(1 \leq p \leq 2)$ :

$$
X_{p}=\left(\oplus_{n \geq 2} \oplus_{A \in \Delta_{n}} \ell_{2}(A)\right)_{p}
$$

We have that $X_{1}$ is isomorphic to a subspace of $\ell_{1}$, while for $1<p<2$ we have that $X_{p}$ is itself isomorphic to $\ell_{p}$ (LINDENSTRAUSS; TZAFRIRI, 1996).

For each $i \geq 2$, let

$$
z_{i}=e_{2 i}-e_{2 i+1}+e_{4 i}+e_{4 i+1}+e_{4 i+2}+e_{4 i+3}
$$

Let $Y_{p} \subset X_{p}$ be the closed subspace spanned by $\left\{z_{i}: i \geq 2\right\}$. 
Theorem 3.4.12 ((LINDENSTRAUSS; TZAFRIRI, 1996), Theorem II.1.g.4). For each $1<$ $p<2$, the space $Y_{p}$ does not have the CAP.

We will now define the spaces we will twist.

Definition 3.4.13. For each $1<p<2$ let $W_{p}=\left(Y_{2}, Y_{1}\right)_{\theta}, \frac{1}{p}=\frac{1-\theta}{2}+\theta$.

We want to apply Theorem 3.2.21, and also determine if the spaces $W_{p}$ have the CAP. We will use the following terminology.

Definition 3.4.14. For $z \in \operatorname{span}\left\{z_{i}\right\}_{i \geq 2}$ we let the $\Delta$-support of $z$ be the set

$$
\left\{A \in \cup_{n} \Delta_{n}: A \cap \operatorname{supp}(z) \neq \emptyset\right\}
$$

Lemma 3.4.15. Let $w \in \operatorname{span}\left\{z_{i}\right\}_{i \geq 2}$ be such that its $\Delta$-support has size $n$. Then

$$
\|w\|_{W_{p}} \leq n^{1-\frac{1}{p}}\|w\|_{Y_{p}}
$$

Proof. Let $f: \mathbb{S} \rightarrow \operatorname{span}\left\{z_{i}\right\}_{i \geq 2}$ be the constant function $f(z)=w$. Then $\|f\|=\|w\|_{Y_{1}} \leq$ $n^{1-\frac{1}{p}}\|w\|_{Y_{p}}$. Therefore, $\|w\|_{W_{p}} \leq n^{1-\frac{1}{p}}\|w\|_{Y_{p}}$.

Proposition 3.4.16. For each $1<p<2$, the space $W_{p}$ does not have the CAP.

Proof. The proof follows the lines of the proof that $Y_{p}$ does not have the CAP from (LINDENSTRAUSS; TZAFRIRI, 1996).

Each $z_{i}$ has $\Delta$-support of size at most six, and therefore, by Lemma 3.4.15, $\left\|z_{i}\right\|_{W_{p}} \leq$ $6^{1-\frac{1}{p}}\left\|z_{i}\right\|_{Y_{p}}$. So $\left(z_{i}\right)$ is bounded in $W_{p}$.

For $i \geq 2$ let

$$
z_{i}^{*}=\frac{e_{2 i}^{*}-e_{2 i+1}^{*}}{2}
$$

We have that $Y_{1} \subset Y_{2}$ with norm 1 , and $\operatorname{span}\left\{z_{i}\right\}_{i \geq 2}$ is dense in $Y_{1}$. This, together with Proposition 2.3.11, implies that $\operatorname{span}\left\{z_{i}\right\}_{i \geq 2}$ is dense in $W_{p}$, and therefore $\left(z_{i}^{*}\right)$ is $w^{*}$-null.

We also have that $z_{i}^{*}\left(z_{i}\right)=1$ for every $i$, so that we have 1 . from Proposition 3.4.9.

For an operator $T: W_{p} \rightarrow W_{p}$ let

$$
\alpha_{n}(T)=2^{-n} \sum_{i \in \sigma_{n}} z_{i}^{*}\left(T z_{i}\right)
$$

Then, following (LINDENSTRAUSS; TZAFRIRI, 1996), for $n \geq 2$ we have

$$
\alpha_{n}(T)-\alpha_{n-1}(T)=2^{-n-1} \sum_{A \in \nabla_{n+1}} \mathbb{E}\left(\sum_{j \in A} \theta_{j} e_{j}^{*}\right) T\left(\sum_{j \in A} \theta_{j} y_{j}\right)
$$

where the expectation is over all choices of signs $\theta_{j}= \pm 1, j \in A$, and

$$
y_{j}=\sum_{k=1}^{9} \lambda_{j, k} e_{f_{k}(j)}
$$

where $j \geq 8$, the functions $f_{k}$ are from 3.4.10, and $\lambda_{j, k}$ are such that $\left|\lambda_{j, k}\right|=1$ for 8 values of $k$, and is $\left|\lambda_{j, k}\right|=2$ for the ninth value.

Let $\frac{1}{p}+\frac{1}{q}=1$. 
Since $Y_{p} \subset X_{p}$, we have by interpolation a norm 1 inclusion $W_{p} \subset X_{p}$. Therefore, using parts 2. and 3. of Lemma 3.4.11, for $n \geq 2$ and $A \in \nabla_{n+1}$ we have

$$
\left\|\sum_{j \in A} \theta_{j} e_{j}^{*}\right\|_{W_{p}^{*}} \leq\left\|\sum_{j \in A} \theta_{j} e_{j}^{*}\right\|_{X_{p}^{*}}=(\# A)^{\frac{1}{q}} \leq\left(2 m_{n+1}\right)^{\frac{1}{q}}
$$

Let $E_{n}=\left\{\sum_{j \in A} \theta_{j} y_{j}: A \in \nabla_{n+1}, \theta_{j}= \pm 1\right\}$. Using the fact that each $A \in \nabla_{n+1}$ has size at least $m_{n+1}$, we have

$$
\begin{aligned}
\left|\alpha_{n}(T)-\alpha_{n+1}(T)\right| & \leq 2^{-n-1} \sum_{A \in \nabla_{n+1}}\left(2 m_{n+1}\right)^{\frac{1}{q}} \sup \left\{\|T x\|: x \in E_{n}\right\} \\
& \leq 2^{\frac{1}{q}} m_{n+1}^{-\frac{1}{p}} \sup \left\{\|T x\|: x \in E_{n}\right\}
\end{aligned}
$$

Then, if we let $F_{n}=2^{\frac{1}{q}} m_{n+1}^{-\frac{1}{p}} E_{n}$, we obtain 3. from Proposition 3.4.9. To obtain, 2., notice that

$$
\sum_{j \in A} \theta_{j} y_{j}=\sum_{j \in A} \theta_{j} \sum_{k=1}^{9} \lambda_{j, k} e_{f_{k}(j)}=\sum_{k=1}^{9} \sum_{j \in A} \theta_{j} \lambda_{j, k} e_{f_{k}(j)}
$$

and by 4. of Lemma 3.4.11, the vector $\sum_{j \in A} \theta_{j} y_{j}$ has $\Delta$-support of size at most 9 . Therefore, by Lemma 3.4.15 and 2 and 4. of Lemma 3.4.11,

$$
\begin{aligned}
\left\|\sum_{j \in A} \theta_{j} y_{j}\right\|_{W_{p}} & \leq 9^{\frac{1}{q}}\left\|\sum_{j \in A} \theta_{j} y_{j}\right\|_{Y_{p}} \\
& =9^{\frac{1}{q}}\left\|\sum_{k=1}^{9} \sum_{j \in A} \theta_{j} \lambda_{j, k} e_{f_{k}(j)}\right\|_{Y_{p}} \\
& \leq 9^{\frac{1}{q}} \sum_{k=1}^{9}\left\|\sum_{j \in A} \theta_{j} \lambda_{j, k} e_{f_{k}(j)}\right\| \\
& \leq 2.9^{\frac{1}{q}} \sum_{k=1}^{9}\left\|\sum_{j \in A} \theta_{j} e_{f_{k}(j)}\right\| \\
& \leq 2.9^{\frac{1}{q}} 9(\# A)^{\frac{1}{2}} \\
& \leq 18.9^{\frac{1}{q}}\left(2 m_{n+1}\right)^{\frac{1}{2}} \\
& =18 \sqrt{2.9^{\frac{1}{q}} m_{n+1}^{\frac{1}{2}}}
\end{aligned}
$$

Therefore, $\sup \left\{\|x\|: x \in F_{n}\right\} \leq 2^{\frac{1}{q}} .18 \sqrt{2} .9^{\frac{1}{q}} m_{n+1}^{\frac{1}{2}-\frac{1}{p}}$. Since $1<p<2$, we have that $\frac{1}{2}-\frac{1}{p}<$ 0 , and by 1 . of Lemma 3.4.11, we have

$$
\sup \left\{\|x\|: x \in F_{n}\right\} \leq 18 \sqrt{2} \cdot 9^{\frac{1}{q}}\left(2^{-\left(\frac{n}{8}-2\right)}\right)^{\frac{1}{p}-\frac{1}{2}}
$$

This implies 2. of Proposition 3.4.9, and we obtain that $W_{p}$ does not have the CAP.

Proposition 3.4.17. For each $1<p<2$, the space $d W_{p}$ does not have the CAP. 
Proof. Again, we will use Proposition 3.4.9. For that, we work with the norm of $d W_{p}$ viewed as a quotient of $\mathscr{F}$. The proof follows the same lines of Proposition 3.4.16, so we simply point out what needs to be changed.

Let $\tilde{z_{i}}=\left(z_{i}, 0\right) \in d W_{p}=\left\{\left(f(\theta), f^{\prime}(\boldsymbol{\theta})\right): f \in \mathscr{F}\right\}$. By taking the constant function $f(z)=$ $z_{i}$, we see that

$$
\left\|\tilde{z_{i}}\right\|_{d W_{p}} \leq 6^{1-\frac{1}{p}}\left\|z_{i}\right\|_{Y_{p}}
$$

Let $\tilde{z}_{i}^{*} \in\left(d W_{p}\right)^{*}$ be given by $\tilde{z}_{i}^{*}(x, y)=z_{i}^{*}(x)$. That is, if $q: d W_{p} \rightarrow W_{p}$ is the quotient map, then $\tilde{z}_{i}^{*}=q^{*}\left(z_{i}^{*}\right)$. Since $q^{*}$ is $w^{*}-w^{*}$-continuous, we have that $z_{i}^{*}$ is $w^{*}$-null.

We replace the vector $z_{i}$ and $z_{i}^{*}$ in the proof of Proposition 3.4.16 with the vectors $\tilde{z_{i}}$ and $\tilde{z}_{i}^{*}$, respectively. have

The vectors $\sum \theta_{j} y_{j}$ are replaced by $\sum \theta_{j}\left(y_{j}, 0\right)$. Again, by taking constant functions, we

$$
\left\|\sum \theta_{j} y_{j}\right\|_{d W_{p}} \leq 9^{1-\frac{1}{p}}\left\|\sum \theta_{j} y_{j}\right\|_{Y_{p}}
$$

This is enough to reproduce the proof of Proposition 3.4.16 to prove that $d W_{p}$ does not have the CAP.

Let $\Omega_{p}$ be the quasilinear map associated to $d W_{p}$.

Theorem 3.4.18. Let $1<p<2$, and let $Z$ be a closed subspace of $W_{p}$. Suppose that there is an increasing sequence $(i(n))$ and a sequence $(j(n))$ of naturals such that:

1. $z_{i(n)} \in Z$ for every $n \in \mathbb{N}$;

2. $\operatorname{supp}\left(z_{i(n)}\right) \subset \sigma_{j(n)} \cup \sigma_{j(n)+1}$, for every $n \in \mathbb{N}$;

3. If $n \neq m$, then $j(n) \neq j(m)$ and $j(n) \neq j(m)+1$.

Then $\left.\Omega_{p}\right|_{Z}$ is nontrivial.

Proof. For every $x \in W_{p}$ we have by interpolation that $\|x\|_{X_{p}} \leq\|x\|_{W_{p}}$. Let $x \in Z$ be of the form

$$
\sum_{n=1}^{N} t_{n} z_{i(n)}
$$

Let $1 \leq p \leq 2$. Then, for each $n$, the coefficient $t_{n}$ contributes at least one time for the norm of $x$ in $X_{p}$, so that

$$
\sum_{n=1}^{N}\left|t_{n}\right|^{p} \leq\|x\|_{X_{p}}^{p}
$$

On the other hand, by 2 . we have that different coefficients $t_{n}$ are never in the same element of the $\Delta$-support of $x$. By considering all the possible partitions of the support of $z_{i}$, which corresponds to all the possible partitions of $\{1, \ldots, 6\}$, we have that there is a constant $K_{p}$ such that

$$
\|x\|_{X_{p}}^{p} \leq K_{p} \sum_{n=1}^{N}\left|t_{n}\right|^{p}
$$

Suppose $\|x\|_{X_{p}}=1$, and let

$$
f(z)=\sum_{n=1}^{N}\left|t_{n}\right|^{\frac{p}{p(z)}} \frac{t_{n}}{\left|t_{n}\right|} z_{i(z)}
$$


where $\frac{1}{p(z)}=\frac{1+z}{2}$. Then $f(\theta)=x$, where $\frac{1}{p}=\frac{1+\theta}{2}$. Furthermore

$$
\|f(i t)\|_{Y_{2}} \leq K_{2}^{\frac{1}{2}}\left(\sum_{n=1}^{N}\left|t_{n}\right|^{p}\right)^{\frac{1}{2}}=K_{2}^{\frac{1}{2}}
$$

and

$$
\|f(1+i t)\|_{Y_{1}} \leq K_{1} \sum_{n=1}^{N}\left|t_{n}\right|^{p}=K_{1}
$$

Therefore, $\|x\|_{W_{p}} \leq K\|x\|_{X_{p}}$, where $K=\max \left\{K_{1}, K_{2}^{\frac{1}{2}}\right\}$. We obtain that if $x \in \operatorname{span}\left\{z_{i(n)}\right\}_{n}$ then $\|x\|_{W_{p}} \leq K\|x\|_{X_{p}}$, and therefore $Z$ contains a subspace isomorphic to a closed subspace of $X_{p}$, which in turn is $\ell_{p}$-saturated. This implies that $p_{Z}=p$, and by Theorem 3.2.21 we have that $\left.\Omega_{p}\right|_{Z}$ is nontrivial.

Corollary 3.4.19. For each $1<p<2, d W_{p}$ is a nontrivial extension of $W_{p}$.

Proof. Apply Theorem 3.4.18 with $i(n)=2^{2 n-1}$ and $j(n)=2 n, n \geq 1$.

More twisted sums involving the approximation property may be found in (CASTILLO; MORENO, 2013), (CHEN; ZHENG, 2013), (GODEFROY; SAPHAR, 1989) and (SZANKOWSKI, 2009).

\subsection{Open problems}

Let $f, g: \mathbb{N} \rightarrow(0, \infty)$ and write $f \sim g$ if $0<\liminf \frac{f(n)}{g(n)} \leq \limsup \frac{f(n)}{g(n)}<\infty$.

As mentioned before, the general principle behind our results and those on (CASTILLO; FERENCZI; GONZÁLEZ, 2017) is the following: let $\bar{X}=\left(X_{0}, X_{1}\right)$ be a compatible couple of Banach spaces and consider a $\lambda(., X): \mathbb{N} \rightarrow(0, \infty)$ of Banach spaces such that

( $\lambda 1) \lambda\left(., X_{0}\right) \nsim \lambda\left(., X_{1}\right)$

( $\lambda 2) \lambda\left(., X_{\theta}\right) \sim \lambda\left(., X_{0}\right)^{1-\theta} \lambda\left(., X_{1}\right)^{\theta}$.

Then $\Omega_{\theta}$ is nontrivial. Furthermore, if

( $\lambda 3$ ) $\lambda(., W) \sim \lambda\left(., X_{0}\right)^{1-\theta} \lambda\left(., X_{1}\right)^{\theta}$ for a class of subspaces of $X_{\theta}$ then $\Omega_{\theta}$ is singular.

Problem 3.5.1. For which parameters $\lambda(., X)$ of Banach spaces if we have $(\lambda 1)$ and $(\lambda 2)$ then $\Omega_{\theta}$ is nontrivial, and for which if we also have $(\lambda 3)$ then $\Omega_{\theta}$ is singular?

Examples 3.4.1 and 3.4.2 also suggest the following problem:

Problem 3.5.2. Let $\bar{X}=\left(X_{0}, X_{1}\right)$ be a compatible couple of Banach spaces and suppose that $d X_{t}$ is nontrivial for every $t \in(0,1), t \neq \frac{1}{2}$. Is $d X_{\frac{1}{2}}$ nontrivial?

A study of this problem in particular cases may be found in (CASTILLO et al., 2017a), a joint work with Castillo, Ferenczi and González. 


\section{Part II}

\section{Twisted sums of operator spaces}



In this chapter we present the necessary background on the theory of operator spaces. The acquainted reader may skip it. As general references for the topic we refer to (EFFROS; RUAN, 2000) and (PISIER, 2003).

\subsection{First definitions and examples}

Let $M_{n}$ denote the space of complex square matrices of size $n$.

Definition 4.1.1. An operator space is a vector space $X$ together with a sequence of complete norms $\left(\|\cdot\|_{n}\right)$ on the spaces $M_{n}(X)$ satisfying:

O1 $\|v \oplus w\|_{m+n}=\max \left\{\|v\|_{m},\|w\|_{n}\right\}$

O2 $\|\alpha v \beta\|_{m} \leq\|\alpha\|\|v\|_{m}\|\beta\|$

for all $v \in M_{m}(X), w \in M_{n}(X), \alpha \in M_{m}, \beta \in M_{m}$, where

$$
v \oplus w=\left(\begin{array}{cc}
v & 0 \\
0 & w
\end{array}\right)
$$

The sequence of norms $\left(\|\cdot\|_{n}\right)$ is called an operator space structure (o. s. s., for short) on $X$, or a quantization of $X$. If $X$ is a Banach space, we suppose that $\left\|_{1}\right\|_{1}$ agrees with the norm of $X$.

Observation 4.1.2. If $\|\cdot\|_{1}$ is complete and $\mathrm{O} 1$ and $\mathrm{O} 2$ are satisfied, then $\|\cdot\|_{n}$ is complete for every $n \in \mathbb{N}$. This follows from Lemma 4.2.12.

Also, in $\mathrm{O} 2$ we actually have an equality.

Example 4.1.3. Let $H$ be a Hilbert space. The space $M_{n}(B(H))$ is naturally equipped with a norm by the linear identification $M_{n}(B(H))=B\left(H^{n}\right)$ given by

$$
\left(\begin{array}{cccc}
T_{1,1} & T_{1,2} & \cdots & T_{1, n} \\
T_{2,1} & T_{2,2} & \cdots & T_{2, n} \\
\vdots & \vdots & \ddots & \vdots \\
T_{n, 1} & T_{n, 2} & \cdots & T_{n, n}
\end{array}\right)\left(\begin{array}{c}
h_{1} \\
h_{2} \\
\vdots \\
h_{n}
\end{array}\right)=\left(\begin{array}{c}
\sum_{j=1}^{n} T_{1, j}\left(h_{j}\right) \\
\sum_{j=1}^{n} T_{2, j}\left(h_{j}\right) \\
\vdots \\
\sum_{j=1}^{n} T_{n, j}\left(h_{j}\right)
\end{array}\right)
$$

With this sequence of norms $B(H)$ is an operator space. 
Definition 4.1.4. If $X$ is an operator space and $Y$ is a closed subspace of $X$, the operator space structure of $Y$ as a subspace of $X$ is the one induced by the inclusions $M_{n}(Y) \subset M_{n}(X)$.

Example 4.1.5. By Example 4.1.3, if $X$ is a closed subspace of $B(H)$ for some Hilbert space $H$ then $X$ has a natural operator space structure as a subspace of $B(H)$. Actually, these are all the operator spaces (see Theorem 4.1.11).

The following notation will be useful.

Notation 4.1.6. Let $X$ be a vector space and $A=\left(a_{i, j}\right) \in M_{n}$. By $A \otimes x$ we denote the matrix $\left(a_{i, j} x\right) \in M_{n}(X)$.

Recall that by using the canonical basis of $\ell_{2}$ we may see the space $B\left(\ell_{2}\right)$ as a space of infinite matrices.

Example 4.1.7. [Row Operator Space] Consider the following subspace of $B\left(\ell_{2}\right)$ :

$$
R=\left\{\left(\begin{array}{cccc}
x_{1} & x_{2} & x_{3} & \cdots \\
0 & 0 & 0 & \cdots \\
0 & 0 & 0 & \cdots \\
\vdots & \vdots & \vdots & \ddots
\end{array}\right):\left(x_{i}\right) \in \ell_{2}\right\} \subset B\left(\ell_{2}\right)
$$

By the $C^{*}$-equality $\left\|x x^{*}\right\|_{B\left(\ell_{2}\right)}=\|x\|_{B\left(\ell_{2}\right)}^{2}$ we have that $R$ is isometric to $\ell_{2}$. Thus the inclusion $R \subset B\left(\ell_{2}\right)$ defines an o. s. s. on $\ell_{2}$.

If $\left(e_{n}\right)$ is any orthonormal system in $\ell_{2}$ then

$$
\left\|\sum_{j=1}^{k} A_{j} \otimes e_{j}\right\|_{M_{n}(R)}=\left\|\sum_{j=1}^{k} A_{j} A_{j}^{*}\right\|^{\frac{1}{2}}
$$

for every $A_{1}, \ldots, A_{k} \in M_{n}$.

Example 4.1.8. [Column Operator Space] Consider the following subspace of $B\left(\ell_{2}\right)$ :

$$
C=\left\{\left(\begin{array}{cccc}
x_{1} & 0 & 0 & \ldots \\
x_{2} & 0 & 0 & \ldots \\
x_{3} & 0 & 0 & \ldots \\
\vdots & \vdots & \vdots & \ddots
\end{array}\right):\left(x_{i}\right) \in \ell_{2}\right\} \subset B\left(\ell_{2}\right)
$$

By the $C^{*}$-equality $\left\|x^{*} x\right\|_{B\left(\ell_{2}\right)}=\|x\|^{2}$ we have that $C$ is isometric to $\ell_{2}$. Thus the inclusion $C \subset B\left(\ell_{2}\right)$ defines an o. s. s. on $\ell_{2}$.

If $\left(e_{n}\right)$ is any orthonormal system in $\ell_{2}$ then

$$
\left\|\sum_{j=1}^{k} A_{j} \otimes e_{j}\right\|_{M_{n}(C)}=\left\|\sum_{j=1}^{k} A_{j}^{*} A_{j}\right\|^{\frac{1}{2}}
$$

for every $A_{1}, \ldots, A_{k} \in M_{n}$.

By nature an operator space is a sequence of Banach spaces, so the morphisms of the category of operator spaces must take into account this structure. 
Definition 4.1.9. Let $X$ and $Y$ be operator spaces and $T: X \rightarrow Y$ be a linear map. For each $n \in \mathbb{N}$ the $n$-amplification $T_{n}$ of $T$ is the linear map $T_{n}: M_{n}(X) \rightarrow M_{n}(Y)$ given by

$$
T_{n}\left(\left(x_{i, j}\right)\right)=\left(T\left(x_{i, j}\right)\right)
$$

for every $\left(x_{i, j}\right) \in M_{n}(X)$.

The linear map $T$ is called completely bounded (c. b., for short) if

$$
\|T\|_{c b}=\sup _{n}\left\|T_{n}\right\|<\infty
$$

If $T_{n}$ is an isometry for each $n \in \mathbb{N}$ then $T$ is called $a$ complete isometry and $X$ and $Y$ are said completely isomorphic.

If $T$ is invertible and $T$ and $T^{-1}$ are c. $b$. then $T$ is called a complete isomorphism and $X$ and $Y$ are said completely isomorphic.

Example 4.1.10. Let $e_{n}$ be the canonical basis of $\ell_{2}$, and for each $n \in \mathbb{N}$ let

$$
x_{n}=\left(\begin{array}{cccc}
e_{1} & e_{2} & \cdots & e_{n} \\
0 & 0 & \cdots & 0 \\
\vdots & \vdots & \ddots & \vdots \\
0 & 0 & \cdots & 0
\end{array}\right) \in M_{n}\left(\ell_{2}\right)
$$

and let $y_{n}=\left(x_{n}\right)^{T}$ (the transpose of $x_{n}$ ). Then equations 4.1 and 4.2 imply that $\left\|x_{n}\right\|_{M_{n}(R)}=$ $\left\|y_{n}\right\|_{M_{n}(C)}=\sqrt{n}$ and $\left\|x_{n}\right\|_{M_{n}(C)}=\left\|y_{n}\right\|_{M_{n}(R)}=1$. This shows that $I d: \ell_{2} \rightarrow \ell_{2}$ is not completely bounded either as seen from $R$ into $C$ or as from $C$ into $R$.

Actually, if $T: \ell_{2} \rightarrow \ell_{2}$ is any linear map then $\|T\|_{c b}=\|T\|_{H S}$, the Hilbert-Schmidt norm of $T$, whenever we see $T$ as from $R$ into $C$ or from $C$ into $R$ (see (PISIER, 2003), page 21). In the case $T$ is an isomorphism we have that $\|T\|_{H S}=\infty$.

In Example 4.1.5 we mentioned that operator spaces are actually subspaces of $B(H)$ for some Hilbert space $H$. This is the content of Ruan's Theorem:

Theorem 4.1.11 (Ruan's Theorem, (RUAN, 1988)). Let $X$ be an operator space. Then there is a Hilbert space $H$ and a complete isometry $T: X \rightarrow T(X) \subset B(H)$.

Example 4.1.12. Let $\mathscr{A}$ be a $C^{*}$-algebra, i. e., a Banach algebra with an involution $*$ such that $\left\|x x^{*}\right\|=\left\|x^{*} x\right\|=\|x\|^{2}$ for every $x \in \mathscr{A}$. The spaces $\ell_{\infty}, C(K)$ for a compact topological space $K$, and $B(H)$ for a Hilbert space $H$, are examples of $C^{*}$-algebras.

Then by the Gelfand-Naimark Theorem ((GELFAND; NEUMARK, 1943), Theorem 1 ) there is an isometric mapping $\pi: \mathscr{A} \rightarrow B(H)$, for some Hilbert space $H$, which preserves involution. Therefore, every $C^{*}$-algebra has a natural o. s. s. Furthermore, this structure is independent of $\pi$ ((PISIER, 2003), Proposition 1.5).

\subsection{Operator space analogues of Banach space construc- tions}

We have already seem in Definition 4.1.4 the definition of subspace of an operator space. Now we see more constructions based on those from Banach space theory. 
Definition 4.2.1. Let $X$ and $Y$ be operator spaces. Their $l_{\infty}$-direct sum $X \oplus_{\infty} Y$ is equipped with the o. $s$. s. given by the identification $M_{n}\left(X \oplus_{\infty} Y\right)=M_{n}(X) \oplus_{\infty} M_{n}(Y)$.

Definition 4.2.2. Let $X$ be an operator space and $Y \subset X$ a subspace. The $o$. s. s. of the quotient space $X / Y$ is given by the identifications $M_{n}(X / Y)=M_{n}(X) / M_{n}(Y)$.

Recall that if $X$ is a Banach space the conjugate space $\bar{X}$ is the Banach space which has the same elements of $X$, same norm, same sum, and multiplication by scalar is substituted by $\lambda \cdot x=\bar{\lambda} x$.

If $x \in X$ we often denote the corresponding element in $\bar{X}$ by $\bar{x}$. With this notation multiplication becomes $\lambda \cdot \bar{x}=\overline{\bar{\lambda} x}$.

Definition 4.2.3. Let $X$ be an operator space. The conjugate space $\bar{X}$ is equipped with the $o . s . s$. induced by the identification $M_{n}(\bar{X})=\overline{M_{n}(X)}$, i. e.,

$$
\left\|\left(\overline{x_{i, j}}\right)\right\|=\left\|\left(x_{i, j}\right)\right\|
$$

for every $\left(x_{i, j}\right) \in M_{n}$.

In Banach space theory we have the space of bounded linear operators $B(X, Y)$. In operator space theory we work with the space of completely bounded operators from $X$ into $Y$.

Observation 4.2.4. If $X$ is an operator space, then $M_{n}(X)$ is also an operator space by the identification $M_{m}\left(M_{n}(X)\right)=M_{m n}(X)$.

Definition 4.2.5. Let $X$ and $Y$ be operator spaces. The space $C B(X, Y)$ is the space of completely bounded operators from $X$ into $Y$. With the c. b. norm it is a Banach space, and it has an o. s. $s$. induced by the identification $M_{n}(C B(X, Y))=C B\left(X, M_{n}(Y)\right)$ given by

$$
\left(T_{i, j}\right)(x)=\left(T_{i, j}(x)\right) \in M_{n}(Y)
$$

for every $\left(T_{i, j}\right) \in M_{n}(C B(X, Y))$.

Observation 4.2.6. If $n \in \mathbb{N}$, unless otherwise stated, we consider the space $M_{n}$ with the o. s. s. induced by the identification $M_{n}=B\left(\ell_{2}^{n}\right)$.

A particular case of Definition 4.2.5 is the following:

Definition 4.2.7. Let $X$ be an operator space. The dual space $X^{*}$ is equipped with the o. $s . s$. defined by the identifications $M_{n}\left(X^{*}\right)=C B\left(X, M_{n}\right)$ given by

$$
\left(x_{i, j}^{*}\right)(x)=\left(x_{i, j}^{*} x\right)
$$

for every $\left(x_{i, j}^{*}\right) \in M_{n}\left(X^{*}\right)$.

Duality may be used to define the $\ell_{1}-$ direct sum.

Definition 4.2.8. Let $X$ and $Y$ be operator spaces. Their $\ell_{1}$-direct sum $X \oplus_{1} Y$ is equipped with the o. s. s. induced by the inclusion $X \oplus_{1} Y \subset\left(X^{*} \oplus_{\infty} Y^{*}\right)^{*}$.

Later we will define the other $l_{p}$-direct sums using complex interpolation. 
Example 4.2.9. Consider the canonical identification $j: \overline{\ell_{2}} \rightarrow\left(\ell_{2}\right)^{*}$ given by

$$
j(\bar{x})(y)=\langle y, x\rangle
$$

Then $j: \bar{R} \rightarrow C^{*}$ is a complete isometry, as well as $j: \bar{C} \rightarrow R^{*}$.

Definition 4.2.10. An operator space $X$ is $(\lambda$-)Hilbertian if as a Banach space it is isometric ( $\lambda$-isomorphic) to a Hilbert space.

So $R$ and $C$ are Hilbertian operator spaces which are not selfdual.

Theorem 4.2.11. [(PISIER, 1996), Theorem 1.1] For each index set I there is only one o. s. s. on $\ell_{2}(I)$ up to complety isometry, denoted $\mathrm{OH}(I)$, for which the canonical identification between $\overline{\ell_{2}}(I)$ and $\left(\ell_{2}(I)\right)^{*}$ induces a complete isometry from $\overline{\mathrm{OH}(I)}$ onto $(\mathrm{OH}(\mathrm{I}))^{*}$. have

If $\left(e_{i}\right)$ is an orthonormal system in $\ell_{2}(I)$ then for every finite sequence $\left(A_{i}\right) \subset M_{n}$ we

$$
\left\|\sum_{i} A_{i} \otimes e_{i}\right\|_{M_{n}(O H(I))}=\left\|\sum_{i} A_{i} \otimes \overline{A_{i}}\right\|^{\frac{1}{2}}
$$

where if $A=\left(a_{i, j}\right)$, then $\bar{A}=\left(\overline{a_{i, j}}\right)$.

If $I=\mathbb{N}, O H(I)$ is denoted simply by $O H$, and if $I=\{1, \ldots, n\}, O H(I)$ is denoted $O H_{n}$.

$\mathrm{OH}$ is called the operator Hilbert space.

The operator Hilbert space may be defined via interpolation of operator spaces, which we describe now.

\subsubsection{Complex interpolation}

Lemma 4.2.12. [(EFFROS; RUAN, 2000), page 22] If $X$ is an operator space then for every $\left(x_{i, j}\right) \in M_{n}(X)$ we have

$$
\sup _{i, j}\left\|x_{i, j}\right\| \leq\left\|\left(x_{i, j}\right)\right\| \leq \sum_{i, j}\left\|x_{i, j}\right\|
$$

Let $\bar{X}=\left(X_{0}, X_{1}\right)$ be a compatible couple of Banach spaces which are operator spaces. Lemma 4.2.12 implies that we have continuous linear inclusions $M_{n}\left(X_{0}\right), M_{n}\left(X_{1}\right) \subset \Sigma(\bar{X})^{n^{2}}$. Therefore, $\left(M_{n}\left(X_{0}\right), M_{n}\left(X_{1}\right)\right)$ is also a compatible couple of Banach spaces.

Definition 4.2.13. Let $\bar{X}=\left(X_{0}, X_{1}\right)$ be a compatible couple of operator spaces. The o. s. $s$. on $\mathscr{F}(\bar{X})$ is given by the identification $M_{n}(\mathscr{F}(\bar{X}))=\mathscr{F}\left(M_{n}\left(X_{0}\right), M_{n}\left(X_{1}\right)\right)$. The o. s. s. on $X_{\theta}$, for $0<\theta<1$, is given by the identification $M_{n}\left(X_{\theta}\right)=\left(M_{n}\left(X_{0}\right), M_{n}\left(X_{1}\right)\right)_{\theta}$.

Proposition 4.2.14. Let $\left(X_{0}, X_{1}\right)$ be a compatible couple of operator spaces. Then $\delta_{\theta}: \mathscr{F} \rightarrow X_{\theta}$, the evaluation at $\theta$, is a c. b. operator and $\mathscr{F} / \operatorname{ker}\left(\delta_{\theta}\right)=X_{\theta}$ completely isometrically.

Theorem 4.2.15. [(PISIER, 1996), Proposition 2.1] Let $\left(X_{0}, X_{1}\right)$ and $\left(Y_{0}, Y_{1}\right)$ be compatible couples of operator spaces, and suppose that $T: X_{j} \rightarrow Y_{j}$ are c. b. maps. Then for every $\theta \in(0,1)$ we have that $T: X_{\theta} \rightarrow Y_{\theta}$ is a c. b. map and

$$
\|T\|_{c b: X_{\theta} \rightarrow Y_{\theta}} \leq\|T\|_{c b: X_{0} \rightarrow Y_{0}}^{1-\theta}\|T\|_{c b: X_{1} \rightarrow Y_{1}}^{\theta}
$$

Complex interpolation allows the definition of the $\ell_{p}$-direct sum of operator spaces. 
Definition 4.2.16. Let $X$ and $Y$ be operator spaces. Their $\ell_{p}$-direct sum $X \oplus_{p} Y$ is equipped with the $o$. s. s. given by the identification

$$
X \oplus_{p} Y=\left(X \oplus_{\infty} Y, X \oplus_{1} Y\right)_{\theta}
$$

for $\theta=\frac{1}{p}$

In many instances interpolation of a Banach space with its conjugate dual gives a Hilbert space. The same behaviour happens in operator space theory.

Theorem 4.2.17. [(PISIER, 1996), Corollary 2.4] Suppose that $X$ is an operator space and that there is a bounded injective map $v: \ell_{2} \rightarrow X$ with dense range. We may then view the couple $\left(\overline{X^{*}}, X\right)$ as compatible by the map $j=v \circ i \circ \overline{v^{*}}: \overline{X^{*}} \rightarrow X$, where $i: \overline{\ell_{2}^{*}} \rightarrow \ell_{2}$ is the canonical identification. Then $\left(\overline{X^{*}}, X\right)_{\frac{1}{2}}=O H$ completely isometrically. Moreover, the scalar product on $\left(\overline{X^{*}}, X\right)_{\frac{1}{2}}$ is given by $\left\langle\overline{x^{*}}, i\left(\overline{y^{*}}\right)\right\rangle=y^{*}\left(i\left(\overline{x^{*}}\right)\right)$ on $i\left(\overline{X^{*}}\right)$, which is dense in $\left(\overline{X^{*}}, X\right)_{\frac{1}{2}}$.

Corollary 4.2.18. [(PISIER, 1996), Corollary 2.6] We have completely isometrically

$$
(R, C)_{\frac{1}{2}}=(C, R)_{\frac{1}{2}}=O H
$$

We notice that interpolation of operator spaces also behaves well under duality.

Theorem 4.2.19. [(PISIER, 1996), Theorem 2.2; (BERGH, 1979)] Let $\bar{X}=\left(X_{0}, X_{1}\right)$ be a compatible couple of operator spaces such that $\Delta(\bar{X})$ is dense in $X_{0}$ and in $X_{1}$, and suppose that at least one of the spaces $X_{0}$ or $X_{1}$ is reflexive. Then $\left(X_{0}^{*}, X_{1}^{*}\right)$ is a compatible couple of operator spaces and we have a completely isometric identification

$$
\left(X_{0}, X_{1}\right)_{\theta}^{*}=\left(X_{0}^{*}, X_{1}^{*}\right)_{\theta}
$$

for every $\theta \in(0,1)$.

So, in particular:

Lemma 4.2.20. We have completely isometrically $\overline{(R, C)_{\theta}^{*}}=(R, C)_{1-\theta}$, for every $\theta \in(0,1)$.

\section{3 $\min$ and max operator spaces}

Every Banach space $X$ has a minimal and a maximal o. s. s. denoted $\min (X)$ and $\max (X)$, respectively. This minimality and maximality are in the sense that if $\left(\|\cdot\|_{n}\right)$ is any o. s. s. on $X$ then we have, for every $n \in \mathbb{N}$

$$
\|\cdot\|_{M_{n}(\min (X))} \leq\|\cdot\|_{n} \leq\|\cdot\|_{M_{n}(\max (X))}
$$

The following is the original definition of $\min (X)$.

Proposition 4.3.1. Let $X$ be a Banach space, $n \in \mathbb{N}$ and $x \in M_{n}(X)$. Then:

$$
\|x\|_{M_{n}(\min (X))}=\sup \left\{\left\|f_{n}(x)\right\|_{M_{n}}: f \in X^{*},\|f\| \leq 1\right\}
$$

We have the following characterizations for $\min (X)$ and $\max (X)$, which may be found in (PAULSEN, 1992): 
Proposition 4.3.2. Let $X$ be a Banach space, $n \in \mathbb{N}$ and $x \in M_{n}(X)$. Then:

$$
\|x\|_{M_{n}(\min (X))}=\sup \left\{\left\|\sum_{i, j} \lambda_{i} \mu_{j} x_{i, j}\right\|_{X}: \sum\left|\lambda_{i}\right|^{2}, \sum\left|\mu_{j}\right|^{2} \leq 1\right\}
$$

Proposition 4.3.3. Let $X$ be a Banach space, $n \in \mathbb{N}$ and $x \in M_{n}(X)$. Then:

$$
\|x\|_{M_{n}(\max (X))}=\inf \{\|A\|\|D\|\|B\|: x=A D B\}
$$

where the infimum is over all decompositions $x=A D B$ with $A$ and $B$ scalar matrices (of any dimension), and $D$ is a diagonal matrix with entries in $X$ (of any dimension).

Proposition 4.3.4. [(BLECHER, 1992), Corollary 2.8] Let X be a Banach space. We have completely isometrically $(\min (X))^{*}=\max \left(X^{*}\right)$ and $(\max (X))^{*}=\min \left(X^{*}\right)$.

From Theorem 4.2.17, we have:

Corollary 4.3.5. [(PISIER, 1996), Corollary 2.5$]$ We have $\left(\min \left(\ell_{2}\right), \max \left(\ell_{2}\right)\right)_{\frac{1}{2}}=$ OH completely isometrically.

Proposition 4.3.6. We have completely isometrically

$$
\left(\min \left(\ell_{\infty}\right), \max \left(\ell_{1}\right)\right)_{\frac{1}{2}}=\mathrm{OH}
$$

One interesting property of the min and max o. s. s. is that bounded operators are automatically completely bounded.

Proposition 4.3.7. [(EFFROS; RUAN, 2000), pages 48 and 49] Let $X$ and $Y$ be operator spaces and $T: X \rightarrow Y$ a linear map. Then

$$
\|T: \max (X) \rightarrow Y\|=\|T: X \rightarrow \min (Y)\|=\|T\|
$$

It could happen that a Banach space has only one o. s. s. up to complete isometry. The next proposition tells that this is not the case for infinite dimensional spaces.

Proposition 4.3.8. Let $X$ be an infinite dimensional Banach space. Then $\min (X)$ and $\max (X)$ are not completely isomorphic.

This is proved the following way: first, it is showed that ((PAULSEN, 1992), Proposition 2.7 and 2.8)

$$
\frac{n}{2} \leq\left\|I d: \min \left(\ell_{2}^{n}\right) \rightarrow \max \left(\ell_{2}^{n}\right)\right\|_{c b} \leq n
$$

Then we take a subspace of $X$ of dimension $n$, say $X_{n}$, such that there is a map $u: X \rightarrow \ell_{2}^{n}$ of norm at most $\sqrt{n}$ and such that $\left\|u^{-1}: \ell_{2}^{n} \rightarrow X_{n}\right\| \leq 1$. Then, since $I_{\ell_{2}}=u \tilde{I}_{X} \tilde{u}^{-1}$, we have

$$
\begin{aligned}
\frac{n}{2} & \leq\left\|u: \max (X) \rightarrow \max \left(\ell_{2}\right)\right\|_{c b}\left\|I_{X}: \min (X) \rightarrow \max (X)\right\|_{c b}\left\|u^{-1}: \min \left(\ell_{2}\right) \rightarrow \min (X)\right\|_{c b} \\
& \leq \sqrt{n}\left\|I_{X}: \min (X) \rightarrow \max (X)\right\|_{c b}
\end{aligned}
$$

so that ((PAULSEN, 1992), Corollary 2.13)

$$
\left\|I_{X}: \min (X) \rightarrow \max (X)\right\|_{c b}=\infty
$$

Now, if $T: \min (X) \rightarrow \max (X)$ is an isomorphism such that $T: \min (X) \rightarrow \max (X)$ is completely bounded we would have

$$
\left\|I_{X}: \min (X) \rightarrow \max (X)\right\| \leq\|T: \min (X) \rightarrow \max (X)\|\left\|T^{-1}: \max (X) \rightarrow \max (X)\right\|<\infty
$$

so that there are not complete isomorphisms between $\min (X)$ and $\max (X)$. 


\subsection{Homogeneous operator spaces}

Definition 4.4.1. An operator space $X$ is called $\lambda$-homogeneous if for every $T: X \rightarrow X$ linear we have

$$
\|T\|_{c b} \leq \lambda\|T\|
$$

If $X$ is 1-homogeneous it is said to be homogeneous.

So, from Proposition 4.3.7 we have:

Proposition 4.4.2. For any Banach space $X$ the operator spaces $\min (X)$ and $\max (X)$ are homogeneous.

Another examples are:

Proposition 4.4.3. The operator spaces $R, C$ and $\mathrm{OH}$ are homogeneous.

The following classical result follows from Theorem 4.2.15.

Proposition 4.4.4. Let $\left(X_{0}, X_{1}\right)$ be a compatible couple of operator spaces. If $X_{0}$ and $X_{1}$ are $\lambda$-homogeneous, then $X_{\theta}$ is $\lambda$-homogeneous for every $\theta \in(0,1)$.

Proposition 4.4.5. Let $X$ be a Hilbertian operator space which is $\lambda$-homogeneous. If $Y$ is a closed subspace of $X$ of same density character of $X$ then $Y$ is $\lambda^{2}$-completely isomorphic to $X$.

\subsection{The Haagerup tensor product}

The category of operator spaces has a special tensor product, the Haagerup tensor product.

Definition 4.5.1. Let $X$ be an operator space and $m, n \in \mathbb{N}$. The norm on $M_{m, n}(X)$ is the one given by the natural identification $M_{m, n}(X) \subset M_{\max \{m, n\}}(X)$ as upper-left matrices.

Recall that we have a linear identification $M_{m, n}(X)=M_{m, n} \otimes X$.

Definition 4.5.2. Let $X$ and $Y$ be vector spaces and $m, n, r \in \mathbb{N}$. The matrix inner product

$$
\odot: M_{m, r}(X) \times M_{r, n}(Y) \rightarrow M_{m, n}(X \otimes Y)
$$

is defined in the following way: if $x=\left(x_{i, j}\right) \in M_{m, r}(X)$ and $y=\left(y_{i, j}\right) \in M_{r, n}(Y)$, then

$$
(x \odot y)_{i, j}=\sum_{k=1}^{r} x_{i, k} \otimes y_{k, j}
$$

Notice that this product is matrix multiplication, except that we use tensor product instead of product of scalars.

Definition 4.5.3. Let $X$ and $Y$ be operator spaces. For $u \in M_{n}(X \otimes Y)$ let

$$
\|u\|_{h}=\inf \|x\|\|y\|
$$

where the infimum is over all decompositions $u=x \odot y$, with $x \in M_{n, r}(X), y \in M_{r, n}(Y)$ and $r \in \mathbb{N}$.

The Haagerup tensor product $X \otimes^{h} Y$ is the operator space given by the completion of the spaces $M_{n}(X \otimes Y)$ with respect to $\|\cdot\|_{h}$. 
What is special regarding the Haagerup tensor product is that it is both injective and projective. Since we have not yet defined complete quotient maps, for now we just state the injective part.

Proposition 4.5.4. [(EFFROS; RUAN, 2000), Proposition 9.2.5] Let $X, Y, X^{\prime}$ and $Y^{\prime}$ be operator spaces, and $\varphi: X \rightarrow X^{\prime}$ and $\psi: Y \rightarrow Y^{\prime}$. Then if $\varphi$ and $\psi$ are complete isomorphisms (respectively, complete isometries) then so is

$$
\varphi \otimes \psi: X \otimes^{h} Y \rightarrow X^{\prime} \otimes^{h} Y^{\prime}
$$

\subsection{The projective tensor product}

Definition 4.6.1. Let $X$ and $Y$ be operator spaces and $n \in \mathbb{N}$. Let $u \in M_{n}(X \otimes Y)$. The projective tensor norm of $u$ is

$$
\|u\|_{\wedge}=\inf \{\|\alpha\|\|x\|\|y\|\|\beta\|: u=\alpha(x \otimes y) \beta\}
$$

where $x \in M_{p}(X), y \in M_{q}(Y), \alpha \in M_{n, p q}, \beta \in M_{p q, n}$ and $p, q$ are arbitrary.

The projective tensor product $X \hat{\otimes} Y$ is the operator space given by the completion of the spaces $M_{n}(X \otimes Y)$ with respect to $\|.\|_{\wedge}$.

\section{7 $\quad L_{p}$ operator spaces}

The $L_{p}$ spaces have natural o. s. s. which come defined by complex interpolation. These definitions may be found in (PISIER, 1998).

Definition 4.7.1. Let $(S, \mu)$ be a measure space. The o. s. s. on $L_{\infty}(\mu)$ is the minimal one.

Definition 4.7.2. Let $(S, \mu)$ be a measure space. The o. s. s. on $L_{1}(\mu)$ is the maximal one.

Definition 4.7.3. Let $(S, \mu)$ be a measure space. The o. s. s. on $L_{p}(\mu)$ is the one induced by the identification

$$
L_{p}(\mu)=\left(\min \left(L_{\infty}(\mu)\right), \max \left(L_{1}(\mu)\right)\right)_{\theta}
$$

for $\theta=\frac{1}{p}$

More generally, we have the operator spaces $L_{p}(\mu ; X)$.

Notice that if $H$ is a Hilbert space, then $L_{\infty}(\mu ; B(H))$ is a $C^{*}$-algebra, and therefore has a natural o. s. S.

Definition 4.7.4. Let $X$ be an operator space, and suppose $X \subset B(H)$ for some Hilbert space $H$. Then the o. s. s. on $L_{\infty}(\mu ; X)$ is given by the inclusion

$$
L_{\infty}(\mu ; X) \subset L_{\infty}(\mu ; B(H))
$$

Definition 4.7.5. Let $X$ be an operator space. The o. s. s. on $L_{1}(\mu ; X)$ is the one given by the identification

$$
L_{1}(\mu ; X)=L_{1}(\mu) \hat{\otimes} X
$$

Definition 4.7.6. Let $X$ be an operator space and $1<p<\infty$. The o. s. s. on $L_{p}(\mu ; X)$ is the one given by the identification

$$
L_{p}(\mu ; X)=\left(L_{\infty}(\mu ; X), L_{1}(\mu ; X)\right)_{\theta}
$$

with $\theta=\frac{1}{p}$. 


\subsection{Infinite matrices} way.

It will be useful to consider an operator space as a space of infinite matrices in a natural

Recall that fixing the canonical basis of $\ell_{2}$ we may see the space $B\left(\ell_{2}\right)$ as a space of infinite matrices over $\mathbb{C}$. In this way, we also have natural inclusions $M_{n} \subset B\left(\ell_{2}\right)$ by completing a matrix in $M_{n}$ with infinite zeros to the right and to bottom.

Now, let $X$ be an operator space, and let $\mathbb{M}_{\infty}(X)$ be the (linear) space of infinite matrices with entries in $X$. We may see the spaces $M_{n}(X)$ as naturally included in $\mathbb{M}_{\infty}(X)$ too.

Definition 4.8.1. Let $X$ be an operator space. By $\pi_{X}^{n}$ (or simply $\pi^{n}$, if the context is clear) we denote the map $\mathbb{M}_{\infty}(X) \rightarrow M_{n}(X)$ which truncates the entries of the matrix. That is:

$$
\pi_{X}^{n}\left(\left(x_{i, j}\right)_{i, j \in \mathbb{N}}\right)=\left(\left(x_{i, j}\right)_{1 \leq i, j \leq n}\right)
$$

Definition 4.8.2. Let $X$ be an operator space. By $M_{\infty}(X)$ we denote the Banach space of matrices $x \in \mathbb{M}_{\infty}(X)$ for which

$$
\|x\|=\sup _{n}\left\|\pi_{X}^{n}(x)\right\|_{M_{n}(X)}=\lim _{n}\left\|\pi_{X}^{n}(x)\right\|_{M_{n}(X)}<\infty
$$

Definition 4.8.3. Let $X$ be an operator space. By $\mathscr{K}(X)$ we denote the closure of $\cup_{n} M_{n}(X)$ inside $M_{\infty}(X)$.

Observation 4.8.4. For every $x \in \mathscr{K}(X)$ we have $x=\lim _{n \rightarrow \infty} \pi_{X}^{n}(x)$.

Notice that since each space $M_{n}(X)$ is $M_{n} \otimes X$ linearly we have that

$$
\left(\cup M_{n}\right) \otimes X=\cup\left(M_{n} \otimes X\right)
$$

is dense in $\mathscr{K}(X)$. From now on, we let $\mathscr{K}=\overline{\cup M_{n}} \subset B\left(\ell_{2}\right)$.

Definition 4.8.5. Let $X$ be an operator space. The minimal tensor product between $\mathscr{K}$ and $X$ is the Banach space

$$
\mathscr{K} \otimes_{\min } X=\mathscr{K}(X)
$$

Observation 4.8.6. This is a particular case of the more general minimal tensor product of operator spaces, which we will not use in what follows.

\section{$4.9 S_{p}$ operator spaces}

Let $S_{p}^{n}$ be the $p$-Schatten class of dimension $n$ and $X$ be an operator space. In (PISIER, 1998) the spaces $S_{p}^{n}[X]$ are defined.

Definition 4.9.1. Let $X$ be an operator space. Write $S_{\infty}^{n}[X]=M_{n}(X)$ and for $1 \leq p<\infty$ let $S_{p}^{n}[X]$ be as a set $M_{n}(X)$, and equip with the norm

$$
\|x\|=\inf \left\{\|a\|_{S_{2 p}^{n}}\|y\|_{M_{n}(X)}\|b\|_{S_{2 p}^{n}}\right\}
$$

where the infimum is over all representations $x=a y b$ with $a, b \in S_{2 p^{n}}$ and $y \in M_{n}(X)$. 
Proposition 4.9.2. [(PISIER, 1998), Lemma 1.7] Let $X$ and $Y$ be operator spaces. For every $T: X \rightarrow Y$ we have

$$
\|T\|_{c b}=\sup _{n}\left\|T_{n}: S_{p}^{n}[X] \rightarrow S_{p}^{n}[Y]\right\|
$$

In particular, $T$ is a complete isometry if and only if $T_{n}: S_{p}^{n}[X] \rightarrow S_{p}^{n}[Y]$ is an isometry for every $n$.

Proposition 4.9.3. [(PISIER, 1998), Corollary 1.4] Let $\left(X_{0}, X_{1}\right)$ be a compatible couple of operator space. If $1 \leq p_{0}, p_{1} \leq \infty, n \in \mathbb{N}$ and $\theta \in(0,1)$ we have isometrically

$$
S_{p_{\theta}}^{n}\left[X_{\theta}\right]=\left(S_{p_{0}}^{n}\left[X_{0}\right], S_{p_{1}}^{n}\left[X_{1}\right]\right)_{\theta}
$$

where $\frac{1}{p_{\theta}}=\frac{1-\theta}{p_{0}}+\frac{\theta}{p_{1}}$.

Proposition 4.9.4. [(PISIER, 1998), Proposition 2.1] Let $1 \leq p<\infty$, and let $(\Omega, \mathscr{A}, \mu)$ be a measure space. Let also $X$ be an operator space. Then we have isometrically

$$
L_{p}\left(\mu ; S_{p}^{n}\right)=S_{p}^{n}\left[L_{p}(\mu)\right]
$$

and

$$
L_{p}\left(\mu, S_{p}^{n}[E]\right)=S_{p}^{n}\left[L_{p}(\mu ; E)\right]
$$

Proposition 4.9.5. [(PISIER, 1998), (2.10)] Let $p \in[1, \infty)$ and $X_{0}$ and $X_{1}$ be operator spaces. Then

isometrically

$$
S_{p}^{n}\left[X_{0} \oplus_{p} X_{1}\right]=S_{p}^{n}\left[X_{0}\right] \oplus_{p} S_{p}^{n}\left[X_{1}\right]
$$

\subsection{Operator algebras}

Recall that a Banach algebra is a Banach space $X$ endowed with a multiplication . : $X \times X \rightarrow X$ such that $(X,$.$) is an associative algebra and for every x, y \in X$ we have

$$
\|x y\| \leq\|x\|\|y\|
$$

If $H$ is a Hilbert space, $B(H)$ is a Banach algebra with respect to composition of operators.

Definition 4.10.1. Let $X$ be an operator space which is a Banach algebra. If there is a Hilbert space $H$, a subalgebra $Y \subset B(H)$ and a complete isomorphism $T: X \rightarrow Y$ which is an algebra homomorphism then $X$ is said to be an operator algebra.

Theorem 4.10.2 ((BLECHER, 1995)). Let $X$ be an operator space which is a Banach algebra. Denote its multiplication by $m$. Then $X$ is an operator algebra if and only if

$$
m: X \otimes^{h} X \rightarrow X
$$

is completely bounded, i. e., if the maps

$$
m^{(n)}: M_{n}(X) \times M_{n}(X) \rightarrow M_{n}(X)
$$

given by

$$
\left(m^{(n)}(x, y)\right)_{i, j}=\sum_{k=1}^{n} m\left(x_{i, k}, y_{k, j}\right)
$$

for $x, y \in M_{n}(X), 1 \leq i, j \leq n, n \in \mathbb{N}$, are uniformly bounded. 
Theorem 4.10.3 ((BLECHER; RUAN; SINCLAIR, 1990), Corollary 3.2). Let $X$ be an operator algebra and $I \subset X$ a closed two sided ideal. Then $X / I$ is an operator algebra with respect to the quotient multiplication.

Theorem 4.10.4 ((BLECHER; LE MERDY, 1995)). Let $\left(X_{0}, X_{1}\right)$ be a compatible couple of operator spaces, and suppose that their sum space is endowed with a multiplication which makes $X_{0}$ and $X_{1}$ operator algebras. Then the restriction of this multiplication on $X_{\theta}$ makes $X_{\theta}$ an operator algebra for every $\theta \in(0,1)$. 


\section{ABSTRACT THEORY OF TWISTED SUMS OF OPERATOR SPACES}

In this chapter we present the abstract theory of twisted sums of operator spaces, modeled after the theory for Banach spaces.

\subsection{First properties}

Let us begin with a few remarks on twisted sums of Banach spaces. Recall that if we have a short exact sequence of (quasi-)Banach spaces and operators

$$
0 \longrightarrow Y \stackrel{i}{\longrightarrow} X \stackrel{q}{\longrightarrow} Z \longrightarrow 0
$$

then the open mapping theorem implies that $i$ is an isomorphism onto its image and $q$ induces an isomorphism between $X / i(Y)$ and $Z$. The same is not true if we are dealing with short exact sequences of operator spaces and completely bounded operators. Consider the following examples.

Example 5.1.1. [(WOOD, 1999 (PhD thesis))] Let $X$ be an infinite dimension Banach space. Then we have a short exact sequence of operator spaces and c. b. maps

$$
0 \longrightarrow 0 \longrightarrow \max (X) \stackrel{I d_{X}}{\longrightarrow} \min (X) \longrightarrow 0
$$

But by Proposition 4.3.8, $I d_{X}$ does not induce a complete isomorphism between $\max (X)$ and $\min (X)$.

Example 5.1.2. Let $X$ be an infinite dimension Banach space. Then we have a short exact sequence of operator spaces and c. b. maps

$$
0 \longrightarrow \max (X) \stackrel{I d_{X}}{\longrightarrow} \min (X) \longrightarrow 0 \longrightarrow 0
$$

But by Proposition 4.3.8, $I d_{X}$ is not a complete isomorphism onto its image.

So, in order to work properly with twisted sums of operator spaces together with short exact sequences we must consider these drawbacks. This motivates the following definition. 
Definition 5.1.3. [(WOOD, 1999 (PhD thesis))] An extension sequence is a short exact sequence of operator spaces and c. b. maps

$$
0 \longrightarrow Y \stackrel{i}{\longrightarrow} X \stackrel{q}{\longrightarrow} Z \longrightarrow 0
$$

where $i$ is a complete isomorphism between $Y$ and $i(Y)$, and q induces a complete isomorphism between $X / i(Y)$ and $Z$.

Definition 5.1.4. Let $Y$ and $Z$ be operator spaces. $A$ complete twisted sum of $Y$ and $Z$ is an operator space $X$ such that $X$ has a completely isomorphic copy of $Y$ and $X / Y \cong Z$ completely isomorphically. If $Y=Z$, we say that $X$ is a complete extension of $Y$.

The following follows from the definitions.

Proposition 5.1.5. Let $X$ be an operator space. Then $X$ is a complete twisted sum of the operator spaces $Y$ and $Z$ if and only if there is an extension sequence

$$
0 \longrightarrow Y \stackrel{i}{\longrightarrow} X \stackrel{q}{\longrightarrow} Z \longrightarrow 0
$$

The quotient map appearing in the definition of a complete extension sequence appears in the literature with the following terminology:

Definition 5.1.6. A surjective c. $b$. map $q: X \rightarrow Z$ between operator spaces is said to be a complete surjection if it induces a complete isomorphism $\tilde{q}$ between $X / \operatorname{ker}(q)$ and $Z$.

Observation 5.1.7. Notice that $\tilde{q}$ is always completely bounded and bijective. So the question is if its inverse is completely bounded.

In (WOOD, 1999 ( $\mathrm{PhD}$ thesis)) $q$ is said to have the complete isomorphism property.

We shall use the following characterizations, which may be found in (PISIER, 2003), after Definition 2.4.3.

Proposition 5.1.8. [(PISIER, 2003)] Let $T: X \rightarrow Y$ be a c. b. map between operator spaces.

1. $T$ is a complete isomorphism into its image if and only if $I d \otimes T: \mathscr{K} \otimes_{\min } X \rightarrow \mathscr{K} \otimes_{\min } Y$ is an isomorphism into its image.

2. $T$ is a complete surjection if and only if $I d \otimes T: \mathscr{K} \otimes_{\min } X \rightarrow \mathscr{K} \otimes_{\min } Y$ is surjective.

The next proposition shows that every complete twisted sums gives rise to a twisted sum of Banach spaces in a natural way.

Proposition 5.1.9. Every extension sequence

$$
0 \longrightarrow Y \stackrel{i}{\longrightarrow} X \stackrel{q}{\longrightarrow} Z \longrightarrow 0
$$

induces a short exact sequence of Banach spaces

$$
0 \longrightarrow \mathscr{K} \otimes_{\min } Y \stackrel{I d \otimes i}{\longrightarrow} \mathscr{K} \otimes_{\min } X \stackrel{I d \otimes q}{\longrightarrow} \mathscr{K} \otimes_{\min } Z \longrightarrow 0
$$


Proof. By Proposition 5.1.8 we have that $I d \otimes i$ is an isomorphism onto its image and $I d \otimes q$ is surjective and bounded. We must show that $\operatorname{ker}(\operatorname{Id} \otimes q)=\operatorname{Im}(\operatorname{Id} \otimes i)$.

Let $y \in \mathscr{K} \otimes_{\min } Y$. We have $y=\lim \pi^{n}(y)$ (see Definition 4.8.1). Then

$$
\begin{aligned}
(I d \otimes q)(I d \otimes i)(y) & =\lim _{n}(\operatorname{Id} \otimes q)(I d \otimes i)\left(\pi^{n}(y)\right) \\
& =\lim _{n} q_{n} \circ i_{n}(y) \\
& =0
\end{aligned}
$$

So we obtain that $\operatorname{Im}(\operatorname{Id} \otimes i) \subset \operatorname{ker}(I d \otimes q)$.

For the reverse inclusion, let $x \in \operatorname{ker}(I d \otimes q)$. For each $n \in \mathbb{N}$ we have

$$
\begin{aligned}
0 & =\pi^{n}(I d \otimes q)(x) \\
& =(I d \otimes q)\left(\pi^{n}(x)\right)
\end{aligned}
$$

So, there is $y_{n} \in M_{n}(Y)$ such that $i_{n}\left(y_{n}\right)=\pi^{n}(x)$. Let us see that $y_{n}$ is a Cauchy sequence in $\mathscr{K} \otimes_{\min } Y$. Indeed, since $i$ is a complete isomorphism, there is $C>0$ such that for every $m, n \in \mathbb{N}$ we have

$$
\begin{aligned}
\left\|y_{n}-y_{m}\right\| & \leq C\left\|(I d \otimes i)\left(y_{n}-y_{m}\right)\right\| \\
& =C\left\|\pi^{n}(x)-\pi^{m}(x)\right\|
\end{aligned}
$$

and since $\pi^{n}(x) \rightarrow x$ we have that $\left(y_{n}\right)$ is Cauchy, and therefore converges to $y \in \mathscr{K} \otimes_{\min } Y$. It follows that

$$
\begin{aligned}
(I d \otimes i)(y) & =\lim (I d \otimes i)\left(y_{n}\right) \\
& =\lim \pi^{n}(x) \\
& =x
\end{aligned}
$$

and therefore $x \in \operatorname{Im}(\operatorname{Id} \otimes i)$.

In the category of operator spaces we do not have an analogue of the Open Mapping Theorem. However, the next proposition shows that in suitable situations we have that a completely bounded operator is automatically a complete isomorphism.

Proposition 5.1.10 (3-lemma for operator spaces). Suppose we have a commutative diagram

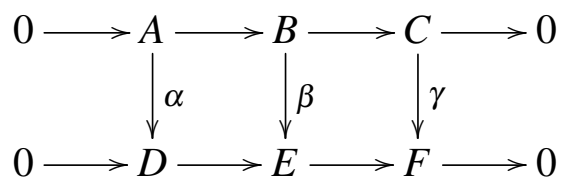

where the rows are extension sequences and $\alpha, \beta$ and $\gamma$ are c. b. maps.

1. If $\alpha$ and $\gamma$ are complete surjections, so is $\beta$.

2. If $\alpha$ and $\gamma$ are complete isomorphisms, so is $\beta$.

Proof. By Proposition 5.1.9 we have a commutative diagram of Banach spaces and operators

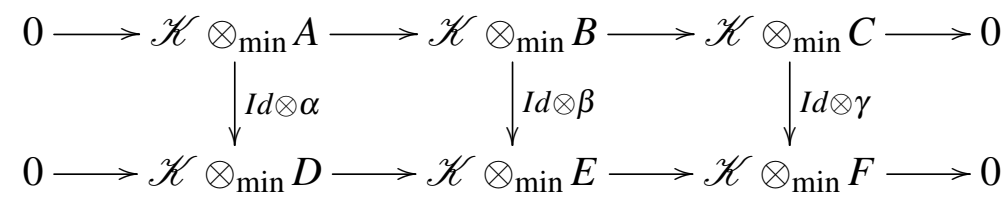

The result now follows from the 3-Lemma (Lemma 2.2.17) and Proposition 5.1.8. 
Recall that the notion of equivalence of twisted sums translates the idea of the subspaces under consideration being essentially in the same manner inside the twisted sums.

Definition 5.1.11. Suppose $X_{i}$ is a complete twisted sum of $Y_{i}$ and $Z_{i}, i=1,2$, and that we have a commutative diagram

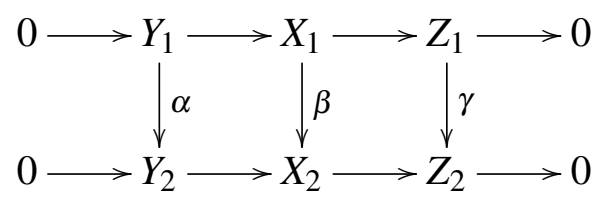

The extension sequences defining $X_{1}$ and $X_{2}$ are

(1) completely isomorphically equivalent, if $\alpha, \beta$ and $\gamma$ are complete isomorphisms;

If $Y_{1}=Y_{2}$ and $Z_{1}=Z_{2}$,

(2) completely projectively equivalent, if $\alpha$ and $\gamma$ are multiples of the identity and $\beta$ is a complete isomorphism;

(3) completely equivalent, if $\alpha=I d_{Y_{1}}, \gamma=I d_{Z_{1}}$, and $\beta$ is a complete isomorphism.

Definition 5.1.12. Let $Y$ and $Z$ be operator spaces. The completely trivial twisted sum of $Y$ and $Z$ is the complete twisted sum $Y \oplus_{\infty} Z$, with the natural inclusion and quotient map.

A complete twisted sum $X$ of $Y$ and $Z$ is completely trivial or completely splits if its extension sequence is completely equivalent to the extension sequence $Y \rightarrow Y \oplus_{\infty} Z \rightarrow Z$.

Observation 5.1.13. In the definition of completely trivial twisted sum we could have used any of the spaces $Y \oplus_{p} Z, p \in[1, \infty]$.

The following proposition may be found essentially in (WOOD, 1999 ( $\mathrm{PhD}$ thesis)), Lemma 3.2.6. We note that his proof does not use the 3-lemma 5.1.10.

Proposition 5.1.14. Consider an extension sequence

$$
0 \longrightarrow Y \stackrel{i}{\longrightarrow} X \stackrel{q}{\longrightarrow} Z \longrightarrow 0
$$

The following are equivalent:

1. $X$ is a completely trivial twisted sum of $Y$ and $Z$;

2. q has a c. b. section;

3. i has a c. b. retraction.

Proof. 1. $\Rightarrow$ 2. Suppose $T: X \rightarrow Y \oplus_{\infty} Z$ is a complete isomorphism making the following diagram commute:

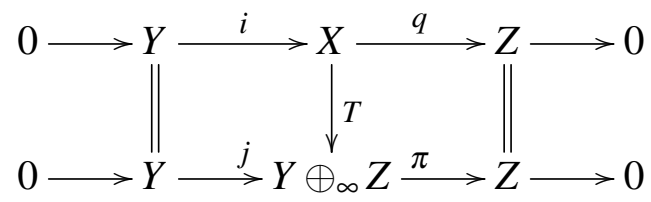

where $j$ and $\pi$ are the natural inclusion and projection. Let $i_{Z}: Z \rightarrow Y \oplus Z$ be given by $z \mapsto(y, z)$, and take $s=T^{-1} \circ i_{Z}$. Then $s$ is linear and completely bounded, and for all $z \in Z$ we have:

$$
q \circ s(z)=q \circ T^{-1} \circ i_{Z}(z)=\pi \circ T \circ T^{-1}(0, z)=z
$$

Therefore, $q \circ s=I d_{Z}$. 
2. $\Rightarrow 3$. Let $s$ be a completely bounded section for $q$ and let $r: X \rightarrow Y$ be given by $r=i^{-1}\left(I d_{X}-s q\right)$. Since for every $x \in X$ we have $q(x-s q(x))=0, r$ is well-defined and is a linear completely bounded map. Besides, for every $y \in Y$,

$$
r \circ i(y)=i^{-1}(i(y)-s q(i(y)))=y
$$

Therefore, $r \circ i=I d_{Y}$.

3. $\Rightarrow 1$. Let $r: X \rightarrow Y$ be a completely bounded retraction of $Y$. Let $T: X \rightarrow Y \oplus Z$ be given by $T(x)=(r(x), q(x))$. Then it is clear that $T$ is completely bounded. Also, $T$ makes the following diagram commute:

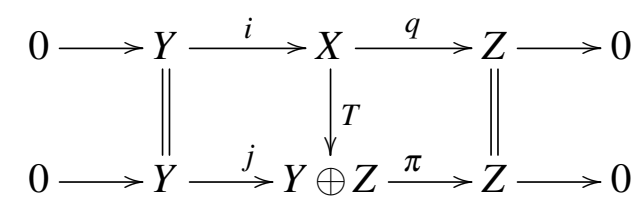

where $j$ and $\pi$ are the natural inclusion and projection. Indeed, for all $y \in Y$

$$
T \circ i(y)=(r \circ i(y), q \circ i(y))=(y, 0)=j(y)
$$

and for all $x \in X$

$$
\pi \circ T(x)=\pi(r(x), q(x))=q(x)
$$

By the 3-Lemma 5.1.10, $T$ is a complete isomorphism, and $X$ is completely trivial.

Notice that being completely trivial is much stronger than being trivial: if $X$ is a complete twisted sum of $Y$ and $Z$ with inclusion $i: Y \rightarrow X$, being completely trivial means that there is a completely bounded projection $P: X \rightarrow i(Y)$. So complete triviality implies triviality. The next proposition shows two situations where the concepts are equivalent.

Proposition 5.1.15. For the following extension sequences triviality and complete triviality are equivalent:

1.

$$
0 \longrightarrow \min (Y) \stackrel{i}{\longrightarrow} X \stackrel{q}{\longrightarrow} Z \longrightarrow 0
$$

2.

$$
0 \longrightarrow Y \stackrel{i}{\longrightarrow} X \stackrel{q}{\longrightarrow} \max (Z) \longrightarrow 0
$$

Proof. For 1., notice that if $r: X \rightarrow \min (X)$ is a bounded retraction for $i$, then by Proposition 4.3.7 $r$ is completely bounded, and then by Proposition 5.1.14 $X$ is completely trivial. A similar argument proves 2 .

\subsection{Twisted sums of the matrices spaces}

Suppose that we have a short exact sequence of Banach spaces

$$
0 \longrightarrow Y \stackrel{i}{\longrightarrow} X \stackrel{q}{\longrightarrow} Z \longrightarrow 0
$$

which are also operator spaces. Then, by Lemma 4.2.12, for every $n \in \mathbb{N}$ we have a short exact sequence

$$
0 \longrightarrow M_{n}(Y) \stackrel{i_{n}}{\longrightarrow} M_{n}(X) \stackrel{q_{n}}{\longrightarrow} M_{n}(Z) \longrightarrow 0
$$


Proposition 5.2.1. Suppose we have a short exact sequence of Banach spaces

$$
0 \longrightarrow Y \stackrel{i}{\longrightarrow} X \stackrel{q}{\longrightarrow} Z \longrightarrow 0
$$

and that $Y, X$ and $Z$ are operator spaces. Then, for every $n \in \mathbb{N}$, (5.1) is trivial if and only if the short exact sequence

$$
0 \longrightarrow M_{n}(Y) \stackrel{i_{n}}{\longrightarrow} M_{n}(X) \stackrel{q_{n}}{\longrightarrow} M_{n}(Z) \longrightarrow 0
$$

is trivial.

Proof. If (5.1) is trivial there is a bounded retraction $r: X \rightarrow Y$ for $i$, and then $r_{n}: M_{n}(X) \rightarrow M_{n}(Y)$ is a bounded retraction for $i_{n}$. This proves the only if part.

We prove now the if part. If $M_{n}(X)$ is trivial then there is a retraction $r: M_{n}(X) \rightarrow M_{n}(Y)$ for $i_{n}$. Let $i_{1,1}: X \rightarrow M_{n}(X)$ be inclusion in the first entry and $q_{1,1}: M_{n}(Y) \rightarrow Y$ be projection of the first entry.

Then $\tilde{r}: X \rightarrow Y$ given by $\tilde{r}=q_{1,1} \circ r \circ i_{1,1}$ is bounded linear and for every $y \in X$ we have

$$
\tilde{r} \circ i(y)=y
$$

and therefore $\tilde{r}$ is a bounded retraction for $i$.

Let $F: Z \rightarrow Y$ be a quasilinear map defining (5.1). Then it may be checked that $F_{n}$ : $M_{n}(Z) \rightarrow M_{n}(Y)$ defined by

$$
F_{n}(z)=\left(F\left(z_{i, j}\right)\right)_{i, j}
$$

for $z \in M_{n}(Z)$ is a quasilinear map defining (5.2), and if $T: Z \rightarrow Y$ is a linear map then

$$
\left\|F_{n}-T_{n}\right\| \leq n^{2}\|F-T\|
$$

For example, let $X$ be an operator space and $F: X \rightarrow X$ be a linear map, and suppose that $F$ is bounded but is not completely bounded. Taking $T=0$, we have

$$
\left\|F_{n}\right\| \rightarrow \infty
$$

\subsection{Completely stricly singular and completely strictly cosingular maps}

As in the study of twisted sums of Banach spaces, we shall be interested in complete twisted sums which are the least completely trivial possible. This suggests the definition of completely strictly singular and completely strictly cosingular maps, which to the best of our knowledge are ours and first presented in (CORREAA, 2018a).

Proposition 5.3.1. Suppose we have an extension sequence

$$
0 \longrightarrow Y \stackrel{i}{\longrightarrow} X \stackrel{q}{\longrightarrow} Z \longrightarrow 0
$$

and that $W$ is a closed subspace of $Z$. Let $p=\left.q\right|_{q^{-1}(W)}$. Then the following is an extension sequence

$$
0 \longrightarrow Y \stackrel{i}{\longrightarrow} q^{-1}(W) \stackrel{p}{\longrightarrow} W \longrightarrow 0
$$


Proof. Since $q \circ i=0$ and $0 \in W$ we have that $i(Y) \subset q^{-1}(W)$. By the continuity of $q$ we also have that $q^{-1}(W)$ is closed, and $p$ is clearly surjective.

We have $\operatorname{ker}(p)=\operatorname{ker}(q)=\operatorname{Im}(i)$, so that the sequence is exact.

We already know that $i$ is a complete isomorphism onto its image. All that remains is to prove that $p$ induces a complete isomorphism $\tilde{p}: q^{-1}(W) / i(Y) \rightarrow W$.

We already know that $\tilde{p}$ is completely bounded. Given $n \in \mathbb{N}$ and $w \in M_{n}(W)$ take $x \in M_{n}(X)$ such that $q_{n}(x)=w$. Then $x \in M_{n}\left(q^{-1}(W)\right)$ and we have

$$
\begin{aligned}
\left\|(\tilde{p})_{n}\left(x+i_{n}(Y)\right)\right\| & =\left\|(\tilde{q})_{n}\left(x+i_{n}(Y)\right)\right\| \\
& \leq C\left\|x+i_{n}(Y)\right\|_{M_{n}(X) / i_{n}(Y)} \\
& =C\left\|x+i_{n}(Y)\right\|_{M_{n}\left(q^{-1}(W)\right) / i_{n}(Y)}
\end{aligned}
$$

for some constant $C>0$, so that $\tilde{p}$ is a complete surjection and the sequence is an extension sequence.

Definition 5.3.2. Suppose we have an extension sequence

$$
0 \longrightarrow Y \stackrel{i}{\longrightarrow} X \stackrel{q}{\longrightarrow} Z \longrightarrow 0
$$

and that $W$ is a closed subspace of $Z$. Let $p=\left.q\right|_{q^{-1}(W)}$. The extension sequence induced by $W$ is

$$
0 \longrightarrow Y \stackrel{i}{\longrightarrow} q^{-1}(W) \stackrel{p}{\longrightarrow} W \longrightarrow 0
$$

Following the Banach space setting, we make the following definitions.

Definition 5.3.3. A c. b. map $T: X \rightarrow Y$ between operator spaces is completely strictly singular (c. s. s., for short) if when restricted to a closed infinite dimensional subspace of $X$ it is never a complete isomorphism onto its image.

The set of c. s. s. maps from $X$ into $Y$ will be denoted $C S S(X, Y)$.

Definition 5.3.4. An extension sequence

$$
0 \longrightarrow Y \stackrel{i}{\longrightarrow} X \stackrel{q}{\longrightarrow} Z \longrightarrow 0
$$

will be called completely singular if $q$ is $c . s . s$.

Definition 5.3.5. A c. b. map $T: X \rightarrow Y$ between operator spaces is completely stricly cosingular (c. s. c., for short) if whenever $E$ is an operator space and $q: Y \rightarrow E$ is a complete surjection such that $q \circ T$ is a complete surjection, it follows that $E$ is finite dimensional.

The set of c. s. c. maps from $X$ into $Y$ will be denoted $C S C(X, Y)$.

Definition 5.3.6. An extension sequence

$$
0 \longrightarrow Y \stackrel{i}{\longrightarrow} X \stackrel{q}{\longrightarrow} Z \longrightarrow 0
$$

will be called completely cosingular if $i$ is $c . s . c$.

Example 5.3.7. Consider $I d_{\ell_{2}}: \max \left(\ell_{2}\right) \rightarrow \min \left(\ell_{2}\right)$. Then $I d_{\ell_{2}}$ is an isomorphism and is completely bounded. Also, $I d$ is c. s. s. Indeed, any subspace of a minimal operator space is minimal, and since $\max \left(\ell_{2}\right)$ is a homogeneous separable Hilbertian operator space, any of its closed infinite dimensional subspaces is completely isomorphic to $\max \left(\ell_{2}\right)$, by Proposition 4.4.5. 
Since $\max \left(\ell_{2}\right)$ and $\min \left(\ell_{2}\right)$ are not completely isomorphic we have that $\left.I d_{\ell_{2}}\right|_{W}$ is never a complete isomorphism for any $W \subset \ell_{2}$ closed infinite dimensional, so $I d_{\ell_{2}}$ is completely strictly singular.

This shows that if $T$ is c. s. s. it does not follows that $T$ is strictly singular.

Also, $I d_{\mathscr{K}} \otimes I d_{\ell_{2}}: \mathscr{K} \otimes_{\min } \max \left(\ell_{2}\right) \rightarrow \mathscr{K} \otimes_{\min } \min \left(\ell_{2}\right)$ is not strictly singular, since it is an isomorphism when restricted to $M_{n}\left(\ell_{2}\right)$ for every $n$, by Lemma 4.2.12.

So, $T$ being c. s. s. is not equivalent to $I d_{\mathscr{K}} \otimes T$ being strictly singular.

Observation 5.3.8. The same considerations show that

$$
C S S\left(\max \left(\ell_{2}\right), \min \left(\ell_{2}\right)\right)=C B\left(\max \left(\ell_{2}\right), \min \left(\ell_{2}\right)\right)
$$

Actually, for any two separable homogeneous Hilbertian operator spaces $X$ and $Y$ which are not completely isomorphic we have $C S S(X, Y)=C B(X, Y)$.

Example 5.3.9. From Example 5.3.7 and Proposition 5.3.13 below it will follow that for any two separable homogeneous Hilbertian operator spaces $X$ and $Y$ which are not completely isomorphic we have $C S C(X, Y)=C B(X, Y)$.

Again, consider $I d_{\ell_{2}}: \max \left(\ell_{2}\right) \rightarrow \min \left(\ell_{2}\right)$ and let $p_{1,1}: \mathscr{K} \otimes \min \left(\ell_{2}\right) \rightarrow \ell_{2}$ be projection of the upmost left coordinate, i. e. $p_{1,1}\left(x_{i, j}\right)=x_{1,1}$.

Then $p_{1,1}$ and $p_{1,1} \circ\left(I d_{\mathscr{K}} \otimes I d_{\ell_{2}}\right): \mathscr{K} \otimes_{\min } \max \left(\ell_{2}\right) \rightarrow \ell_{2}$ are quotient maps, so that $I d_{\mathscr{K}} \otimes I d_{\ell_{2}}$ is not strictly cosingular.

This reasoning shows that $T$ being c. s. c. is not equivalent to $I d_{\mathscr{K}} \otimes T$ being strictly cosingular. Also, it follows that if $\operatorname{Im}(T)$ is infinite dimensional then $\operatorname{Id} \mathscr{K} \otimes T$ is not strictly cosingular.

We may characterize completely singular extension sequences by means of partial sections of the quotient map.

Proposition 5.3.10. Suppose that we have an extension sequence

$$
0 \longrightarrow Y \stackrel{i}{\longrightarrow} X \stackrel{q}{\longrightarrow} Z \longrightarrow 0
$$

Then the following are equivalent:

1. There is a closed infinite dimensional subspace $W$ of $Z$ such that the extension sequence induced by $W$ is completely trivial.

2. There is a closed infinite dimensional subspace $X_{0}$ of $X$ such that $\left.q\right|_{X_{0}}$ is a complete isomorphism onto its image.

Proof. 1. $\Rightarrow$ 2. Suppose that

$$
0 \longrightarrow Y \stackrel{i}{\longrightarrow} q^{-1}(W) \stackrel{p}{\longrightarrow} W \longrightarrow 0
$$

is completely trivial, where $p=\left.q\right|_{q^{-1}(W)}$. Then, by Proposition 5.1.14, there is a completely bounded section $s$ for $p$.

Let $X_{0}=s(W)$. We will show that $\left.q\right|_{X_{0}}$ is a complete isomorphism.

Indeed, $\left.q\right|_{X_{0}}$ is completely bounded, and $\left.q\right|_{X_{0}} \circ s=\left.I d\right|_{W}$. On the other hand, given $x \in X_{0}$, we have that there is $w \in W$ so that $s(w)=x$. Therefore

$$
s \circ q(x)=s q s(w)=s(w)=x
$$


so that $\left.s \circ q\right|_{X_{0}}=I d_{X_{0}}$, and since $s$ is completely bounded, $\left.q\right|_{X_{0}}$ is a complete isomorphism between $X_{0}$ and $W$.

2. $\Rightarrow 1$. Let $X_{0} \subset X$ be a closed infinite dimensional subspace such that $\left.q\right|_{X_{0}}$ is a complete isomorphism onto its image, say, $W=q\left(X_{0}\right)$.

Then the extension sequence

$$
0 \longrightarrow Y \stackrel{i}{\longrightarrow} q^{-1}(W) \stackrel{p}{\longrightarrow} W \longrightarrow 0
$$

is completely trivial. Indeed, if we let $s_{1}=\left(\left.q\right|_{X_{0}}\right)^{-1}: W \rightarrow X_{0}$, and let $j: X_{0} \rightarrow q^{-1}(W)$ be the inclusion then $s=j \circ s_{1}$ is a completely bounded section for $p=\left.q\right|_{q^{-1}(W)}$, and the extension sequence is completely trivial by Proposition 5.1.14.

Corollary 5.3.11. An extension sequence

$$
0 \longrightarrow Y \stackrel{i}{\longrightarrow} X \stackrel{q}{\longrightarrow} Z \longrightarrow 0
$$

is completely singular if and only if for every closed infinite dimensional subspace $W \subset Z$ the induced extension sequence is nontrivial.

The next result implies that complete singularity and complete cosingularity are preserved by the different notions of equivalence of complete twisted sums.

Proposition 5.3.12. Let $X$ and $Y$ be operator spaces. The classes $\operatorname{CSS}(X, Y)$ and $\operatorname{CSC}(X, Y)$ are closed by composition on the left and on the right with c. b. maps.

Proof. The proof is divided in four parts:

(a) If $T \in C S S(X, Z)$ and $S \in C B\left(X^{\prime}, X\right)$, then $T \circ S \in C S S\left(X^{\prime}, Z\right)$

Take a closed subspace $W$ of $X^{\prime}$ such that $\left.(T \circ S)\right|_{W}$ is a complete isomorphism onto its image. This means that there is $C>0$ such that for all $w \in M_{n}(W), n \in \mathbb{N}$,

$$
\left\|S_{n}(w)\right\| \leq\|S\|_{c b}\|w\| \leq C\|S\|_{c b}\left\|T_{n} \circ S_{n}(w)\right\| \leq C\|T\|_{c b}\|S\|_{c b}\left\|S_{n}(w)\right\|
$$

So $\left.T\right|_{S(W)}: S(W) \rightarrow Z$ is a complete isomorphism onto its image, and since $T(S(W))$ is closed, so is $S(W)$. Since $T$ is c. s. s., $S(W)$ is finite dimensional.

But $\left.(T \circ S)\right|_{W}$ is injective, and therefore so is $\left.S\right|_{W}$. Thus $W$ is finite dimensional.

(b) If $T \in C S S(X, Z)$ and $S \in C B\left(Z, Z^{\prime}\right)$, then $S \circ T \in C S S\left(X, Z^{\prime}\right)$

Let $W$ be a closed subspace of $X$ such that $\left.(S \circ T)\right|_{W}$ is a complete isomorphism. There is a constant $C>0$ such that for every $w \in M_{n}(W), n \in \mathbb{N}$,

$$
\|w\| \leq C\left\|S_{n} \circ T_{n}(w)\right\| \leq C\|S\|_{c b}\left\|T_{n}(w)\right\|
$$

that is, $T$ is a complete isomorphism on $W$, and so $W$ is finite dimensional.

(c) If $T \in \operatorname{CSC}(Y, X)$ and $S \in C B\left(Y^{\prime}, Y\right)$, then $T \circ S \in C S C\left(Y^{\prime}, X\right)$

Let $E$ be an operator space and let $q: X \rightarrow E$ and $p^{\prime}: Y^{\prime} \rightarrow E$ be complete quotient maps such that $q \circ T \circ S=p^{\prime}$.

Then the map $p=q \circ T: Y \rightarrow E$ is a complete quotient map. Indeed, surjectivity follows from $p \circ S$ being surjective, and it is a c. b. map by composition. We must prove that there is a constant $C>0$ such that for every $y \in M_{n}(Y), n \in \mathbb{N}$,

$$
\left\|y+\operatorname{ker} p_{n}\right\| \leq C\left\|p_{n}(y)\right\|
$$


Given $y \in M_{n}(Y)$ take $y^{\prime} \in M_{n}\left(Y^{\prime}\right)$ such that $p_{n}^{\prime}\left(y^{\prime}\right)=p_{n}(y)$. Since $p^{\prime}=p \circ S$ and $p^{\prime}$ is a complete quotient map there is $K>0$ such that:

$$
\begin{aligned}
\left\|y+\operatorname{ker} p_{n}\right\| & =\left\|S_{n}\left(y^{\prime}\right)+\operatorname{ker} p_{n}\right\| \\
& =\inf \left\{\left\|S_{n}\left(y^{\prime}\right)+\tilde{y}\right\|: p_{n}(\tilde{y})=0\right\} \\
& \leq \inf \left\{\left\|S_{n}\left(y^{\prime}\right)+S_{n}\left(y^{\prime \prime}\right)\right\|: p_{n}^{\prime}\left(y^{\prime \prime}\right)=0\right\} \\
& \leq\|S\|_{c b} \inf \left\{\left\|y^{\prime}+y^{\prime \prime}\right\|: p_{n}^{\prime}\left(y^{\prime \prime}\right)=0\right\} \\
& \leq K\|S\|_{c b}\left\|p_{n}^{\prime}\left(y^{\prime}\right)\right\| \\
& =K\|S\|_{c b}\left\|p_{n}(y)\right\|
\end{aligned}
$$

So $p$ is a complete quotient, and $q \circ T=p$. Since $T$ is c. s. c., $E$ is finite dimensional.

(d) If $T \in C S S(Y, X)$ and $S \in C B\left(X, X^{\prime}\right)$, then $S \circ T \in C S S\left(Y, X^{\prime}\right)$

Let $q^{\prime}: X^{\prime} \rightarrow E$ and $p: Y \rightarrow E$ be complete quotient maps such that $q^{\prime} \circ S \circ T=p$. First, we prove that $q=q^{\prime} \circ S: X \rightarrow E$ is a complete quotient map.

Since $p=q \circ T, q$ is surjective. We must show that there is $C>0$ such that for all $x \in M_{n}(X), n \in \mathbb{N}$,

$$
\left\|x+\operatorname{ker} q_{n}\right\| \leq C\left\|q_{n}(x)\right\|
$$

Given $x \in M_{n}(X)$ take $y \in M_{n}(Y)$ such that $q_{n}\left(T_{n}(y)\right)=p_{n}(y)=q_{n}(x)$. Since $p$ is a complete quotient there is $K>0$ such that

$$
\begin{aligned}
\left\|x+\operatorname{ker} q_{n}\right\| & =\left\|T_{n}(y)+\operatorname{ker} q_{n}\right\| \\
& =\inf \left\{\left\|T_{n}(y)+\tilde{x}\right\|: q_{n}(\tilde{x})=0\right\} \\
& \leq \inf \left\{\left\|T_{n}(y)+T_{n}\left(y^{\prime}\right)\right\|: q_{n}\left(T_{n}\left(y^{\prime}\right)\right)=0\right\} \\
& \leq\|T\|_{c b} \inf \left\{\left\|y+y^{\prime}\right\|: p_{n}\left(y^{\prime}\right)=0\right\} \\
& \leq K\|T\|_{c b}\left\|p_{n}(y)\right\| \\
& =K\|T\|_{c b}\left\|q_{n}(x)\right\|
\end{aligned}
$$

So $q$ is a complete quotient, and $q \circ T=p$. Since $T \in C S C(Y, X), E$ is finite dimensional.

The next proposition will be useful in proving the complete cosingularity of complete twisted sums.

Proposition 5.3.13. Let $X$ and $Y$ be operator spaces, and $T \in C B(X, Y)$.

1. If $T^{*} \in \operatorname{CSC}\left(Y^{*}, X^{*}\right)$ then $T \in C S S(X, Y)$.

2. If $T^{*} \in \operatorname{CSS}\left(Y^{*}, X^{*}\right)$ then $T \in C S C(X, Y)$.

Proof. . 1. Take a closed subspace $W$ of $X$ such that $\left.T\right|_{W}$ is a complete isomorphism and let $i: W \rightarrow X$ be the inclusion. Then $T \circ i=j$ is a complete isomorphic injection.

We have $i^{*} \circ T^{*}=j^{*}$, and $i^{*}: X^{*} \rightarrow W^{*}$ and $j^{*}: Y^{*} \rightarrow W^{*}$ are complete quotient maps. But $T^{*} \in \operatorname{CSC}\left(Y^{*}, X^{*}\right)$, thus $W^{*}$ is finite dimensional, and so is $W$.

2. Let $q: Y \rightarrow E$ and $p: X \rightarrow E$ be complete quotient maps such that $q \circ T=p$.

Then $T^{*} \circ q^{*}=p^{*}$, and $q^{*}: E^{*} \rightarrow Y^{*}$ and $p^{*}: E^{*} \rightarrow X^{*}$ are complete isomorphisms onto their images. So $\left.T^{*}\right|_{q^{*}\left(E^{*}\right)}$ is a complete isomorphism. But $q^{*}\left(E^{*}\right)$ is closed in $Y^{*}$ and $T^{*} \in \operatorname{CSS}\left(Y^{*}, X^{*}\right)$, so $q^{*}\left(E^{*}\right)$ is finite dimensional, and so is $E$. 


\subsection{Categorical constructions}

As in the theory of twisted sums of Banach spaces we may apply the pullback and pushout constructions to the theory of twisted sums of operator spaces. These objects have quite natural operator space structures, as we shall see, and may be found in (PAULSEN, 1998) (the proofs presented here are original, as far as we can tell).

\subsubsection{Pullback}

Definition 5.4.1. Let $X, Y$ and $Z$ be operator spaces and $A: X \rightarrow Z$ and $B: Y \rightarrow Z$ be bounded linear maps. The pullback of $\{A, B\}$ is the space

$$
\Xi=\left\{(x, y) \in X \oplus_{\infty} Y: A x=B y\right\}
$$

with the o. s. s. induced as a subspace of $X \oplus_{\infty} Y$.

We have operators $\pi_{X}: \Xi \rightarrow X$ and $\pi_{Y}: \Xi \rightarrow Y$, which are simply the restrictions of the natural projections from $X \oplus_{\infty} Y$ onto $X$ and $Y$, respectively. Then $\left\|\pi_{X}\right\|_{c b}=\left\|\pi_{Y}\right\|_{c b}=1$.

The pullback is characterized by the property that it makes the following diagram commute:

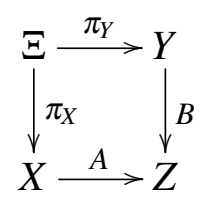

That is, if $U$ is an operator space and $u_{X}: U \rightarrow X$ and $u_{Y}: U \rightarrow Y$ are c. b. operators such that the following diagram commutes

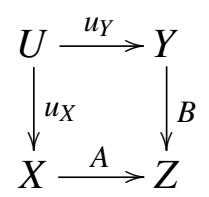

then there is a unique c. b. operator $T: U \rightarrow \Xi$ such that $\pi_{X} \circ T=u_{X}$ and $\pi_{Y} \circ T=u_{Y}$.

Suppose we have a diagram of Banach spaces and operators

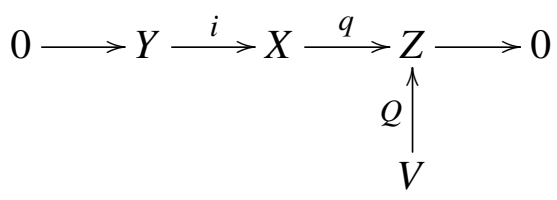

where the first line is exact and $Q$ is surjective. We can then consider the pullback of $\{q, Q\}$ and obtain a commutative diagram (CABELLO SÁNCHEZ; CASTILLO, 2000):

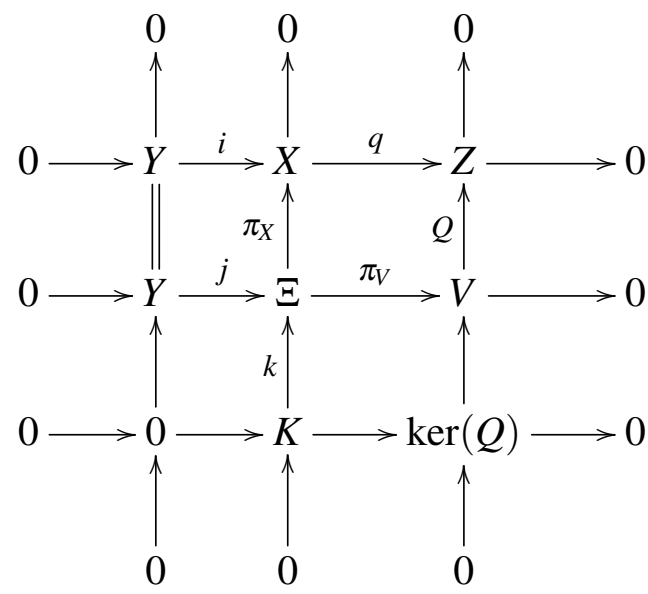


Let us suppose that the spaces in (5.3) are endowed with o. s. s. Then $K=\operatorname{ker}\left(\pi_{X}\right)$ has the operator space structure induced by $\Xi$.

Proposition 5.4.2. Suppose we have a diagram induced by pullback like (5.4).

1. The third line is an extension sequence, with $K$ completely isometric to $\operatorname{ker}(Q)$.

2. If $Q$ is c.b. and the first line is an extension sequence then the second line is an extension sequence.

3. If $q$ is c.b. and the third column is an extension sequence then the second column is an extension sequence.

Proof. 1. We have

$$
K=\operatorname{ker}\left(\pi_{X}\right)=\{(0, v) \in \Xi\}=\left\{(0, v) \in X \oplus_{\infty} V: Q(v)=0\right\}
$$

which is naturally completely isometric to $\operatorname{ker}(Q)$.

2. If $i_{X}: X \rightarrow X \oplus_{\infty} V$ is the natural inclusion then $j=i_{X} \circ i$, so that it is a complete isomorphism onto its image.

Also, $\pi_{V} \circ j=0$, and if $(x, v) \in \operatorname{ker}\left(\pi_{V}\right)$ then $v=0$ and $q(x)=0$, which implies that $x=i(y)$ and therefore $(x, v)=j(y)$, so that $\operatorname{Im}(j)=\operatorname{ker}\left(\pi_{V}\right)$.

Given $v \in V$ take $x \in X$ such that $q(x)=Q(v)$. Then $(x, v) \in \Xi$ and $\pi_{V}(x, v)=v$, so that $\pi_{V}$ is surjective.

All that remains is to show that $\pi_{V}$ induces a complete isomorphism $\tilde{\pi}_{V}$ between $\Xi / j(Y)$ and $V$.

Since $\pi_{V}$ is completely bounded we have that $\tilde{\pi}_{V}$ is completely bounded. Now, let $(x, v) \in M_{n}(\Xi)$. Since $q$ induces a complete isomorphism there is $C>0$ independent of $x, v$ and $n$ such that

$$
\begin{aligned}
\left\|(x, v)+j_{n}(Y)\right\| & =\inf \left\{\left\|\left(x+i_{n}(y), v\right)\right\|: y \in M_{n}(Y)\right\} \\
& \leq \inf \left\{\left\|x+i_{n}(y)\right\|_{M_{n}(X)}: y \in M_{n}(Y)\right\}+\|v\|_{M_{n}(V)} \\
& \leq C\left\|q_{n}(x)\right\|_{M_{n}(Z)}+\|v\|_{M_{n}(V)} \\
& =C\left\|Q_{n}(v)\right\|_{M_{n}(Z)}+\|v\|_{M_{n}(V)} \\
& \leq\left(1+C\|Q\|_{c b}\right)\|v\|_{M_{n}(V)} \\
& =\left(1+C\|Q\|_{c b}\right)\left\|\tilde{\pi_{V}}\left((x, v)+j_{n}(Y)\right)\right\|_{M_{n}(V)}
\end{aligned}
$$

so that $\tilde{\pi}_{V}$ is a complete isomorphism.

3. is proved in a similar way.

\subsubsection{Pushout}

Definition 5.4.3. Let $Y, X$ and $K$ be operator spaces and let $A: Y \rightarrow X$ and $B: Y \rightarrow K$ be bounded linear maps. Let $D=\left\{(A y,-B y) \in X \oplus_{\infty} K: y \in Y\right\}$ and $\Delta=\bar{D}$. The pushout space of $\{A, B\}$ has a natural o. s. s. defined by the identification

$$
\mathscr{P} \mathscr{O}=\left(X \otimes_{\infty} K\right) / \Delta
$$

If $A$ or $B$ is an isomorphism then $D=\Delta$. Also, we have bounded operators $j_{X}: X \rightarrow \mathscr{P} \mathscr{O}$ and $j_{K}: K \rightarrow \mathscr{P} \mathscr{O}$ given by $j_{X}(x)=(x, 0)+\Delta$ and $j_{K}(k)=(0, k)+\Delta$, and the pushout space is 
characterized by the fact that it makes the following diagram commute:

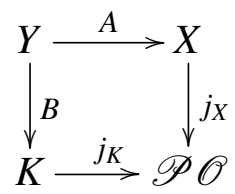

That is, if $U$ is an operator space and $u_{X}: X \rightarrow U$ and $u_{K}: K \rightarrow U$ are c. b. operators making the following diagram commute

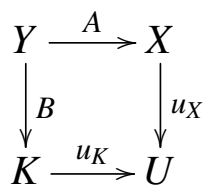

then there is a unique c. b. operator $T: \mathscr{P} \mathscr{O} \rightarrow U$ such that $T \circ j_{X}=u_{X}$ and $T \circ j_{K}=u_{K}$.

Proposition 5.4.4. Let $Y, X$ and $K$ be operator spaces and let $A: Y \rightarrow X$ and $B: Y \rightarrow K$ be bounded linear maps. The maps $j_{X}: X \rightarrow \mathscr{P} \mathscr{O}$ and $j_{K}: K \rightarrow \mathscr{P} \mathscr{O}$ into the pushout space of $\{A, B\}$ are completely bounded.

Proof. Let $n \in \mathbb{N}$ and $x \in M_{n}(X)$. We have

$$
\begin{aligned}
\left\|\left(j_{X}\right)_{n}(x)\right\| & =\left\|(x, 0)+M_{n}(\Delta)\right\| \\
& =\inf \left\{\|\left(x+A_{n} y,-B_{n} y\right): y \in M_{n}(y)\right\} \\
& \leq\|(x, 0)\| \\
& =\|x\|
\end{aligned}
$$

So that $\left\|j_{X}\right\|_{c b} \leq 1$. In the same way we prove that $\left\|j_{K}\right\|_{c b} \leq 1$.

Proposition 5.4.5. Suppose we have a diagram

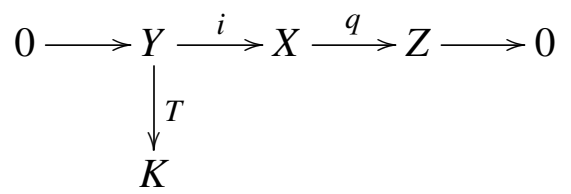

where $Y, X$ and $Z$ are operator spaces, the line is an extension sequence and $T$ is $c . b$. Then the pushout of $\{i, T\}$ induces a commutative diagram

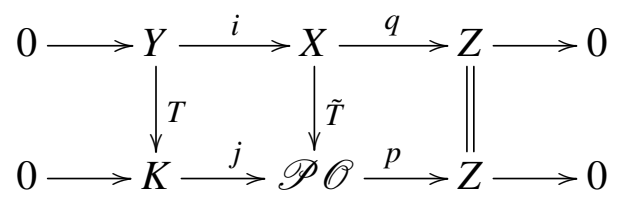

where $p$ is defined by $p((x, k)+\Delta)=q(x), j=j_{K}, \tilde{T}=j_{X}$, and the second line is an extension sequence.

Proof. We already know from the Banach space case that the sequences are exact and that the diagram commutes. We must prove that the second line is an extension sequence.

1. $j$ is a complete isomorphism onto its image

We have from Proposition 5.4.4 that $j$ is completely bounded. Let $n \in \mathbb{N}$ and $k \in M_{n}(K)$. Let $C_{1}>0$ be such that $\|y\| \leq C_{1}\left\|i_{n} y\right\|$ for every $y \in M_{n}(Y)$, independent of $n$. Then, we have for every $y \in M_{n}(Y)$

$$
\|k\| \leq\left\|k-T_{n} y\right\|+\left\|T_{n} y\right\|
$$




$$
\begin{aligned}
& \leq\left\|k-T_{n} y\right\|+C_{1}\|T\|_{c b}\left\|i_{n} y\right\| \\
& \leq\left(1+C_{1}\|T\|_{c b}\right)\left\|\left(i_{n} y, k-T_{n} y\right)\right\|
\end{aligned}
$$

and this implies

$$
\|k\| \leq\left(1+C_{1}\|T\|_{c b}\right)\left\|j_{n} k\right\|
$$

Therefore, $j$ is a complete isomorphism onto its image.

2. $p$ is completely bounded

We have that $p((x, k)+\Delta)=q(x)$. If $n \in \mathbb{N}$ and $(x, k)+M_{n}(\Delta) \in M_{n}(\mathscr{P} \mathscr{O})$ we have $q_{n}(x)=q_{n}\left(x+i_{n}(y)\right)$ for every $y \in M_{n}(Y)$. Therefore

$$
\begin{aligned}
\left\|p_{n}\left((x, k)+M_{n}(\Delta)\right)\right\| & =\left\|q_{n}(x)\right\| \\
& \leq\|q\|_{c b} \inf \left\{\left\|x+i_{n} y\right\|: y \in M_{n}(Y)\right\} \\
& \leq\|q\|_{c b} \inf \left\{\left\|\left(x+i_{n} y, k-T_{n} y\right)\right\|: y \in M_{n}(Y)\right\} \\
& =\|q\|_{c b}\left\|(x, k)+M_{n}(\Delta)\right\|
\end{aligned}
$$

so that $\|p\|_{c b} \leq\|q\|_{c b}$.

3. $p$ is a complete quotient map

Since $p$ is completely bounded we have that $\tilde{p}: \mathscr{P} \mathscr{O} / j(K) \rightarrow Z$ is completely bounded. We must prove that its inverse is also completely bounded.

Let $\left((x, k)+M_{n}(\Delta)\right)+M_{n}(j(K)) \in M_{n}(\mathscr{P} \mathscr{O} / j(K))$. Since $q$ is a complete quotient map there is a constant $C_{2}$ independent of $x, k$ and $n$ such that

$$
\begin{array}{ll} 
& \left\|\left((x, k)+M_{n}(\Delta)\right)+M_{n}(j(K))\right\| \\
= & \inf \left\{\left\|\left(x, k^{\prime}\right)+M_{n}(\Delta)\right\|: k^{\prime} \in M_{n}(K)\right\} \\
=\quad & \inf \left\{\left\|\left(x+i_{n} y, k^{\prime}-T_{n} y\right)\right\|: y \in M_{n}(Y), k^{\prime} \in M_{n}(K)\right\} \\
\leq \quad & \inf \left\{\left\|x+i_{n} y\right\|: y \in M_{n}(Y)\right\} \\
= & \left\|x+i_{n}(Y)\right\| \\
\leq \quad & C_{2}\left\|q_{n} x\right\| \\
= & C_{2}\left\|(\tilde{p})_{n}\left(\left((x, k)+M_{n}(\Delta)\right)+M_{n}(j(K))\right)\right\|
\end{array}
$$

where in the first inequality we took $k^{\prime}=T_{n} y$.

Therefore, $\tilde{p}$ is a complete quotient map.

Observation 5.4.6. The proof of Proposition 5.4.5 shows that if $q$ induces a complete isometry between $X / i(Y)$ and $Z$ then $p$ induces a complete isometry between $\mathscr{P} \mathscr{O} / K$ and $Z$.

Proposition 5.4.7. In (5.5), if $T$ is a complete isomorphism onto its image so is $\tilde{T}$.

Proof. By Proposition 5.4.4 we have that $\tilde{T}$ is completely bounded.

By Proposition 5.4.5 and the 3-Lemma 5.1.10 we have that $\tilde{T}$ is a complete isomorphism. Let us estimate the norm of its inverse.

Let $x \in M_{n}(X)$. We have for every $y \in M_{n}(Y)$ that

$$
\begin{aligned}
\|x\| & \leq\left\|x+i_{n} y\right\|+\left\|-i_{n} y\right\| \\
& \leq\left\|x+i_{n} y\right\|+\|i\|_{c b}\left\|T^{-1}\right\|_{c b}\left\|-T_{n} y\right\| \\
& \leq\left(1+\|i\|_{c b}\left\|T^{-1}\right\|_{c b}\right)\left\|\left(x+i_{n} y,-T_{n} y\right)\right\|
\end{aligned}
$$

Since $Y$ was arbitrary we obtain $\left\|\tilde{T}^{-1}\right\|_{c b} \leq\left(1+\|i\|_{c b}\left\|T^{-1}\right\|_{c b}\right)$. 


\subsection{Complete twisted sums induced by complex interpo- lation}

Now we show how the method to obtain twisted sums from complex interpolation extends to the operator space setting. We notice that interpolation for families of finite dimensional operator spaces is considered in (PISIER, 2008).

Proposition 5.5.1. Suppose we have an interpolation family $\left\{X_{z}: z \in \partial G ; X\right\}$ with containing space $\mathscr{U}$ and containing function $k$.

Suppose that each $X_{z}$ is an operator space and that for every $x \in M_{n}(X)$ the function $\left.t \mapsto\|x\|_{M_{n}\left(X_{e} i t\right.}\right), t \in[0,2 \pi]$, is measurable.

Then for each $n \in \mathbb{N}$ the family $\left\{M_{n}\left(X_{z}\right): z \in \partial G ; M_{n}(X)\right\}$ is an interpolation family with containing space $\mathscr{U}^{n^{2}}$ and containing function $k$.

Proof. We may suppose that $G=\mathbb{D}$.

Let $i_{z}: X_{z} \rightarrow \mathscr{U}$ be the linear continuous injections defining the interpolation family. Then, by Lemma 4.2.12, $\left(i_{z}\right)_{n}: M_{n}\left(X_{z}\right) \rightarrow \mathscr{U}^{n^{2}}$ are also continuous (we identify $\mathscr{U}^{n^{2}}$ with $\left.M_{n}(\mathscr{U})\right)$. Indeed

$$
\begin{aligned}
\left\|\left(i_{z}\right)_{n}(x)\right\| & =\max \left\{\left\|i_{z}\left(x_{i, j}\right)\right\|: 1 \leq i, j \neq n\right\} \\
& \leq\left\|i_{z}\right\| \max \left\{\left\|x_{i, j}\right\|_{z}: 1 \leq i, j \leq n\right\} \\
& \leq\left\|i_{z}\right\|\|x\|_{M_{n}\left(X_{z}\right)}
\end{aligned}
$$

for every $x \in M_{n}\left(X_{z}\right)$. This calculations shows that $\mathscr{U}^{n^{2}}$ is a containing space and that $k$ is a containing function for the family. It remains to show that for every $x \in M_{n}(X)$ the function $t \mapsto\|x\|_{M_{n}\left(X_{e} i t\right.}$ is $\log ^{+}$-integrable.

Let $I=\left\{(i, j): 1 \leq i, j \leq n, x_{i, j} \neq 0\right\}$. By Lemma 2.3.60, given $z_{0} \in G$ we have

$$
\begin{aligned}
\int_{0}^{2 \pi} \log ^{+}\|x\|_{M_{n}\left(X_{e} e^{i t}\right.} P_{z_{0}}\left(e^{i t}\right) d t & \leq \int_{0}^{2 \pi} \log ^{+}\left(\sum_{(i, j) \in I}\left\|x_{i, j}\right\|_{e^{i t}}\right) P_{z_{0}}\left(e^{i t}\right) d t \\
& \leq \int_{0}^{2 \pi} \log \left(1+\sum_{(i, j) \in I}\left\|x_{i, j}\right\|_{e^{i t}}\right) P_{z_{0}}\left(e^{i t}\right) d t \\
& \leq 2 \pi \log (\# I+1)+\sum_{(i, j) \in I} \int_{0}^{2 \pi} \log ^{+}\left\|x_{i, j}\right\|_{e^{i t}} P_{z_{0}}\left(e^{i t}\right) d t \\
& <\infty
\end{aligned}
$$

Definition 5.5.2. Suppose we have an interpolation family $\mathscr{X}=\left\{X_{z}: z \in \partial G ; X\right\}$, that each $X_{z}$ is an operator space and that for every $x \in M_{n}(X)$ the function $t \mapsto\|x\|_{M_{n}\left(X_{e i t}\right)}, t \in[0,2 \pi]$, is measurable. Then the family $\left\{X_{z}: z \in \partial G ; X\right\}$ is called an interpolation family of operator spaces.

We denote $\mathscr{X}_{n}=\left\{M_{n}\left(X_{z}\right): z \in \partial G ; M_{n}(X)\right\}$.

Proposition 5.5.3. Let $\left\{X_{z}: z \in \partial G ; X\right\}$ be an interpolation family of operator spaces and fix $z_{0} \in G$. Let us induce on $M_{n}\left(X_{\left\{z_{0}\right\}}\right)$ (respectively, $M_{n}\left(X_{\left[z_{0}\right]}\right)$ ) the norm given by the identification $M_{n}\left(X_{\left\{z_{0}\right\}}\right)=\left(M_{n}\left(X_{z}\right)\right)_{\left\{z_{0}\right\}}$ (respectively, $\left.M_{n}\left(X_{\left[z_{0}\right]}\right)=\left(M_{n}\left(X_{z}\right)\right)_{\left[z_{0}\right]}\right)$. Then $X_{\left\{z_{0}\right\}}$ and $X_{\left[z_{0}\right]}$ are operator spaces. Also, $\mathscr{F}$ is an operator space with the o. s. s. induced by the identification $M_{n}(\mathscr{F})=\mathscr{F}\left(\mathscr{X}_{n}\right)$. 
Proof. Let $n, m \in \mathbb{N}$ and $x \in M_{n}(X), y \in M_{m}(X)$, and let $f \in \mathscr{G}\left(\mathscr{X}_{n}\right)$ and $g \in \mathscr{G}\left(\mathscr{X}_{m}\right)$ be such that $f\left(z_{0}\right)=x, g\left(z_{0}\right)=y$.

Then the function $h$ given by $h(z)=f(z) \oplus g(z)$ is in $\mathscr{G}\left(\mathscr{X}_{n+m}\right), h\left(z_{0}\right)=x \oplus y$ and $\|h\|=\max \{\|f\|,\|g\|\}$. Since $f$ and $g$ are arbitrary we get

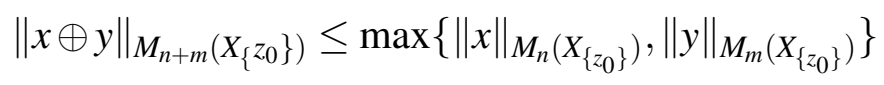

Similarly, given $x \in M_{n}(X), \alpha, \beta \in M_{n}$ and $f \in \mathscr{G}\left(\mathscr{X}_{n}\right)$ such that $f\left(z_{0}\right)=x$, we have that the function $h$ given by $h(z)=\alpha f(z) \beta$ is in $\mathscr{G}\left(\mathscr{X}_{n}\right)$ and $h\left(z_{0}\right)=\alpha x \beta$. Furthermore, $\|h\| \leq$ $\|\alpha\|\|f\|\|\beta\|$, and since $f$ was arbitrary we have

$$
\|\alpha x \beta\|_{M_{n}\left(X_{\left\{z_{0}\right\}}\right)} \leq\|\alpha\|\|x\|_{M_{n}\left(X_{\left\{z_{0}\right\}}\right)}\|\beta\|
$$

If we consider functions in $\mathscr{F}$ we obtain the same estimates for $\left[z_{0}\right]$, and the first part of the result is proved.

For the second part, notice that $\mathscr{G}\left(\mathscr{X}_{n}\right)=M_{n}(\mathscr{G})$ as sets, and check the properties in these dense subsets.

Proposition 5.5.4. Let $\mathscr{X}=\left\{X_{z}: z \in \partial G, X\right\}$ be an interpolation family admissible at $z_{0}$. Then for each $n \in \mathbb{N}$ the interpolation family $\mathscr{X}_{n}$ is admissible at $z_{0}$.

Proof. The proof of Proposition 2.3.62 shows that $\mathscr{X}$ is admissible if and only if $\mathscr{G}(\mathscr{X}) \cap$ $\operatorname{ker}\left(\boldsymbol{\delta}_{z_{0}}\right)$ is dense in $\operatorname{ker}\left(\boldsymbol{\delta}_{z_{0}}\right)$.

Let $f \in \mathscr{F}\left(\mathscr{X}_{n}\right)$ be such that $f\left(z_{0}\right)=0$. We may write $f=\left(f_{i, j}\right)$. Then we have $f_{i, j} \in$ $\mathscr{F}(\mathscr{X})$ and $f_{i, j}\left(z_{0}\right)=0$ for every $i, j$. Given $\varepsilon>0$, we may approximate each $f_{i, j}$ by a function $g_{i, j} \in \mathscr{G} \cap \operatorname{ker}\left(\delta_{z_{0}}\right)$ in such a way that $\left\|f_{i, j}-g_{i, j}\right\|<\frac{\varepsilon}{n^{2}}$.

Then $g=\left(g_{i, j}\right) \in \mathscr{G}\left(\mathscr{X}_{n}\right) \cap \operatorname{ker}\left(\left(\delta_{z_{0}}\right)_{n}\right)$ and $\|f-g\|<\varepsilon$, by Lemma 4.2.12. If we let $\delta_{z_{0}, n}$ denote the evaluation at $z_{0}$ for the family $\mathscr{X}_{n}$ we have that $\delta_{z_{0}, n}=\left(\delta_{z_{0}}\right)_{n}$, so that the result is proved.

Lemma 5.5.5. Suppose we have an interpolation family of operator spaces $\left\{X_{z}: z \in \partial G ; X\right\}$ which is admissible at $z_{0}$. Then $\delta_{z_{0}}^{\prime}: \operatorname{ker}\left(\delta_{z_{0}}\right) \rightarrow X_{z_{0}}$ is completely bounded.

Proof. As in Proposition 5.5.4, if we let $\delta_{z_{0}, n}\left(\delta_{z_{0}, n}^{\prime}\right)$ denote the evaluation (evaluation of the derivative) at $z_{0}$ for the family $\mathscr{X}_{n}$ we have that $\delta_{z_{0}, n}=\left(\delta_{z_{0}}\right)_{n}\left(\delta_{z_{0}, n}^{\prime}=\left(\delta_{z_{0}}^{\prime}\right)_{n}\right.$, respectively).

In Proposition 2.3.63 it is proved that for any interpolation family admissible at $z_{0}$ the evaluation of the derivative at $z_{0}$ restricted to the kernel of the evaluation at $z_{0}$ has norm at most $\left|\varphi^{\prime}\left(z_{0}\right)\right|$, where $\varphi: G \rightarrow \mathbb{D}$ is a conformal map with $\varphi\left(z_{0}\right)=0$.

So $\| \delta_{z_{0}, n}^{\prime}\left|\operatorname{ker}\left(\delta_{z_{0}, n}\right)\right||\leq| \varphi^{\prime}\left(z_{0}\right) \mid$, and therefore $\|\left.\delta_{z_{0}}^{\prime}\left|\operatorname{ker}\left(\delta_{z_{0}}\right)\right|\right|_{c b} \leq\left|\varphi^{\prime}\left(z_{0}\right)\right|$.

Therefore, by Proposition 5.4.5, we may extend the diagram

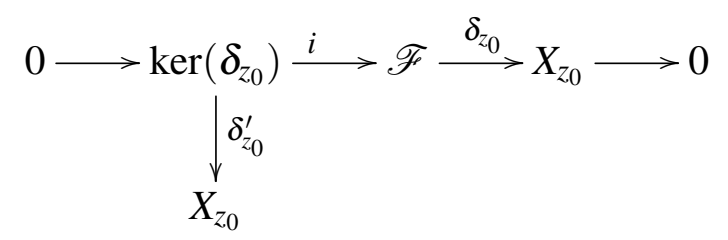

to obtain a complete extension of $X_{z_{0}}$ :

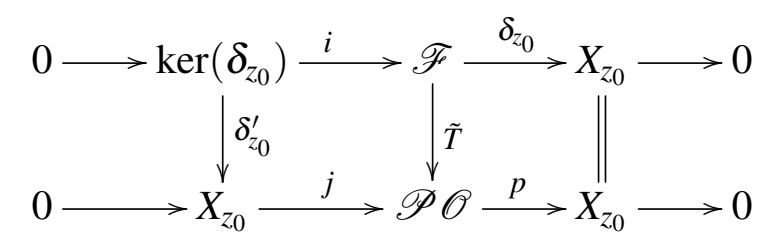


That is, we have a natural o. s. s. on the extension of $X_{z_{0}}$ induced by complex interpolation which makes it a complete extension of $X_{z_{0}}$.

Recall that in the Banach space setting we have another description for the space $\mathscr{P} \mathscr{O}$ of 5.6, given by the space

$$
\mathscr{F} /\left(\operatorname{ker} \delta_{z_{0}} \cap \operatorname{ker} \delta_{z_{0}}^{\prime}\right)
$$

which has a natural o. s. s. as a quotient of $\mathscr{F}$. From now on, we will denote

$$
d X_{z_{0}}=\mathscr{F} /\left(\operatorname{ker} \delta_{z_{0}} \cap \operatorname{ker} \delta_{z_{0}}^{\prime}\right)=\left\{\left(f\left(z_{0}\right), f^{\prime}\left(z_{0}\right)\right): f \in \mathscr{F}\right\}
$$

(recall that in the Banach space case this was reserved for a different description of $\mathscr{P} \mathscr{O}$ ).

Proposition 5.5.6. Suppose we have an interpolation family of operator spaces $\left\{X_{z}: z \in \partial G ; X\right\}$ which is admissible at $z_{0}$. Then we have a complete extension of $X_{z_{0}}$

$$
0 \longrightarrow X_{z_{0}} \stackrel{i}{\longrightarrow} d X_{z_{0}} \stackrel{q}{\longrightarrow} X_{z_{0}} \longrightarrow 0
$$

where $i(y)=(0, y)$ and $q(x, y)=x$.

Proof. $1 . i$ is completely bounded

Let $\varphi: G \rightarrow \mathbb{D}$ be a conformal map with $\varphi\left(z_{0}\right)=0$.

Let $y \in M_{n}\left(X_{z_{0}}\right)$ and take a function $f \in \mathscr{F}\left(\mathscr{X}_{n}\right)$ such that $f\left(z_{0}\right)=y$. Then $g=\frac{\varphi}{\varphi^{\prime}\left(z_{0}\right)} f \in$ $\mathscr{F}\left(\mathscr{X}_{n}\right), f\left(z_{0}\right)=0$ and $f^{\prime}\left(z_{0}\right)=y$. So

$$
\left\|i_{n}(y)\right\|=\|(0, y)\| \leq\|g\|=\frac{\|f\|}{\left|\varphi^{\prime}\left(z_{0}\right)\right|}
$$

Since $f$ was arbitrary we have $\left\|i_{n}(y)\right\| \leq \frac{\|y\|}{\left|\varphi^{\prime}\left(z_{0}\right)\right|}$ and therefore $\|i\|_{c b} \leq \frac{1}{\left|\varphi^{\prime}\left(z_{0}\right)\right|}$.

2. $i$ is a complete isomorphism onto its image

Given $y \in M_{n}(Y)$, let $f \in \mathscr{F}\left(\mathscr{X}_{n}\right)$ be such that $f\left(z_{0}\right)=0, f^{\prime}\left(z_{0}\right)=y$. Since $\mathscr{X}_{n}$ is admissible at $z_{0}$ we have that $g=\varphi^{\prime}\left(z_{0}\right) \frac{f}{\varphi} \in \mathscr{F}\left(\mathscr{X}_{n}\right)$, and $g\left(z_{0}\right)=y$. So

$$
\|y\| \leq\|g\|=\left|\varphi^{\prime}\left(z_{0}\right)\right|\|f\|
$$

Since $f$ was arbitrary we have $\|y\| \leq\left|\varphi^{\prime}\left(z_{0}\right)\right|\|(0, y)\|$, and $i$ is a complete isomorphism onto its image.

3. $q$ is completely bounded

Given $(x, y) \in M_{n}\left(d X_{z_{0}}\right)$ we have

$$
\left\|q_{n}(x, y)\right\|=\|x\| \leq\|(x, y)\|
$$

so that $\|q\|_{c b}=1$.

4. $\tilde{q}$ is a complete isometry

Let $(x, y) \in M_{n}\left(d X_{z_{0}}\right)$. Take $f \in \mathscr{F}\left(\mathscr{X}_{n}\right)$ such that $f\left(z_{0}\right)=x, f^{\prime}\left(z_{0}\right)=y$. Then

$$
\begin{aligned}
\left\|\tilde{q}_{n}\left((x, y)+i_{n}\left(X_{\theta}\right)\right)\right\| & =\|x\| \\
& =\inf \left\{\|f+g\|: g \in \operatorname{ker}\left(\left(\delta_{z_{0}}\right)_{n}\right)\right\} \\
& =\inf \left\{\|h\|: h\left(z_{0}\right)=f\left(z_{0}\right), h^{\prime}\left(z_{0}\right)-f^{\prime}\left(z_{0}\right) \in M_{n}\left(X_{z_{0}}\right)\right\} \\
& =\inf \left\{\|h\|: h\left(z_{0}\right)=x, h^{\prime}\left(z_{0}\right) \in y+M_{n}\left(X_{z_{0}}\right)\right\} \\
& =\left\|(x, y)+j_{n}\left(X_{z_{0}}\right)\right\|
\end{aligned}
$$


Proposition 5.5.7. Suppose we have an interpolation family of operator spaces $\left\{X_{z}: z \in \partial G ; X\right\}$ which is admissible at $z_{0}$. Then we have a complete equivalence of extension sequences

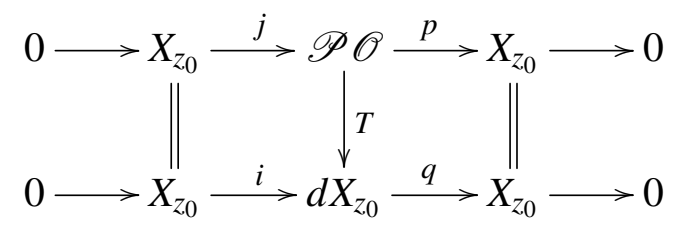

where $T((f, x)+\Delta)=\left(f\left(z_{0}\right), x+f^{\prime}\left(z_{0}\right)\right)$.

Proof. If $x \in X_{z_{0}}$ we have

$$
T \circ j(x)=T((0, x)+\Delta)=(0, x)=i(x)
$$

and if $(f, x)+\Delta \in \mathscr{P} \mathscr{O}$ we have

$$
q \circ T((f, x)+\Delta)=q\left(f\left(z_{0}\right), x+f^{\prime}\left(z_{0}\right)\right)=f\left(z_{0}\right)=p((f, x)+\Delta)
$$

so that the diagram commutes.

Let us show that $T$ is completely bounded.

Then

Let $(f, x)+M_{n}(\Delta) \in M_{n}(\mathscr{P} \mathscr{O})$ and take $g \in \mathscr{F}\left(\mathscr{X}_{n}\right)$ such that $g\left(z_{0}\right)=0$ and $g^{\prime}\left(z_{0}\right)=x$.

$$
T_{n}\left((f, x)+M_{n}(\Delta)\right)=\left(f\left(z_{0}\right), x+f^{\prime}\left(z_{0}\right)\right)=\left((f+g)\left(z_{0}\right),(f+g)^{\prime}\left(z_{0}\right)\right)
$$

so that

$$
\left\|T_{n}\left((f, x)+M_{n}(\Delta)\right)\right\|=\inf \left\{\|f+g+\varphi h\|: h \in \operatorname{ker}\left(\delta_{z_{0}}\right)_{n}\right\}
$$

where $\varphi: G \rightarrow \mathbb{D}$ is a conformal map with $\varphi\left(z_{0}\right)=0$. In turn, we have

$$
\left\|(f, x)+M_{n}(\Delta)\right\|=\inf \left\{\left\|\left(f+l, x-l^{\prime}\left(z_{0}\right)\right)\right\|: l \in \operatorname{ker}\left(\delta_{z_{0}}\right)_{n}\right\}
$$

Given $l \in \operatorname{ker}\left(\delta_{z_{0}}\right)_{n}$ we have that $g-l \in \operatorname{ker}\left(\delta_{z_{0}}\right)_{n}$. Since the family is admissible the function $h=\frac{g-l}{\varphi}$ is in $\mathscr{F}\left(\mathscr{X}_{n}\right)$. Notice that $h\left(z_{0}\right)=\frac{x-l^{\prime}\left(z_{0}\right)}{\varphi^{\prime}\left(z_{0}\right)}$.

So, given $\varepsilon>0$ there is $h_{l, \varepsilon} \in \operatorname{ker}\left(\delta_{z_{0}}\right)_{n}$ such that $\left\|h+h_{l, \varepsilon}\right\| \leq\left\|\frac{x-l^{\prime}\left(z_{0}\right)}{\varphi^{\prime}\left(z_{0}\right)}\right\|+\varepsilon$. Then

$$
\begin{aligned}
\left\|f+g+\varphi h_{l, \varepsilon}\right\| & \leq\|f+l\|+\left\|g-l+\varphi h_{l, \varepsilon}\right\| \\
& =\|f+l\|+\left\|h+h_{l, \varepsilon}\right\| \\
& \leq\|f+l\|+\left\|\frac{x-l^{\prime}\left(z_{0}\right)}{\varphi^{\prime}\left(z_{0}\right)}\right\|+\varepsilon \\
& \leq\left(1+\frac{1}{\left|\varphi^{\prime}\left(z_{0}\right)\right|}\right)\left\|\left(f+l, x-l^{\prime}\left(z_{0}\right)\right)\right\|+\varepsilon
\end{aligned}
$$

Since $l$ and $\varepsilon$ were arbitary

$$
\left\|T_{n}\left((f, x)+M_{n}(\Delta)\right)\right\| \leq\left(1+\frac{1}{\left|\varphi^{\prime}\left(z_{0}\right)\right|}\right)\left\|(f, x)+M_{n}(\Delta)\right\|
$$

so that $\|T\|_{c b} \leq\left(1+\frac{1}{\left|\varphi^{\prime}\left(z_{0}\right)\right|}\right)$.

Now, an application of the 3-Lemma 5.1.10 shows that $T$ is a complete isomorphism. We, however, may obtain a universal bound for the norm of $T^{-1}$. 
We have

$$
\begin{aligned}
\left\|T_{n}\left((f, x)+M_{n}(\Delta)\right)\right\| & =\left\|\left(f\left(z_{0}\right), x+f^{\prime}\left(z_{0}\right)\right)\right\| \\
& =\inf \left\{\|h\|: h\left(z_{0}\right)=f\left(z_{0}\right), h^{\prime}\left(z_{0}\right)=x+f^{\prime}\left(z_{0}\right)\right\}
\end{aligned}
$$

If $h$ is in the set above then $g=h-f \in \operatorname{ker}\left(\delta_{z_{0}}\right)_{n}$ and $g^{\prime}\left(z_{0}\right)=x$, so that $(g,-x) \in M_{n}(\Delta)$. Then

$$
\begin{aligned}
\left\|(f, x)+M_{n}(\Delta)\right\| & \leq\|(f, x)+(g,-x)\| \\
& =\|(h, 0)\| \\
& =\|h\|
\end{aligned}
$$

Since $h$ was arbitrary, we obtain

$$
\left\|(f, x)+M_{n}(\Delta)\right\| \leq\left\|T_{n}\left((f, x)+M_{n}(\Delta)\right)\right\|
$$

so that $\left\|T^{-1}\right\|_{c b} \leq 1$.

If we have a compatible couple $\bar{X}=\left(X_{0}, X_{1}\right)$ of operator spaces and consider it as a family then the compatibility of $\left(M_{n}\left(X_{0}\right), M_{n}\left(X_{1}\right)\right)$ implies that we may see $\bar{X}$ as an interpolation family of operator spaces, and the previous discussion may be adapted to this case to obtain a complete extension $X_{\theta}$.

We now prove the Reiteration Theorem for extensions of operator spaces induced by complex interpolation.

Theorem 5.5.8. Let $\bar{X}=\left(X_{0}, X_{1}\right)$ be a compatible couple of operator spaces, let $\theta_{j} \in[0,1]$, $j=0,1$ and let $Y_{j}=X_{\theta_{j}}$. Consider the compatible couple $\bar{Y}=\left(Y_{0}, Y_{1}\right)$ (with containing space $\Sigma(\bar{X})$ ). If $\Delta(\bar{Y})$ is dense in $Y_{0}, Y_{1}$ and $\Delta(\bar{X})$ then for every $\theta \in(0,1)$ we have

$$
Y_{\theta}=X_{\eta}
$$

completely isometrically, where $\eta=(1-\theta) \theta_{0}+\theta \theta_{1}$.

Furthermore, the extension sequences defining $d Y_{\theta}$ and $d X_{\eta}$ are completely projectively equivalent.

Proof. The first part on reiteration of interpolation is in (PISIER, 1996), Proposition 2.1, and is a consequence of the Reiteration Theorem for complex interpolation of Banach spaces.

For the second part, we find a completely bounded operator $T$ making the following diagram commute:

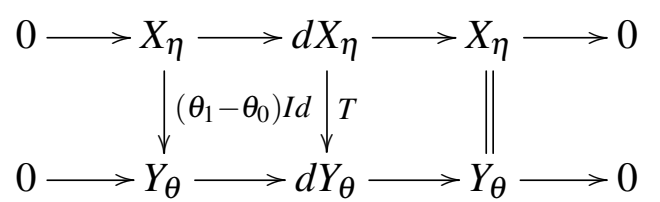

By the 3-Lemma 5.1.10 the extension sequences will be completely projectively equivalent. Let $T$ be given by $T(x, y)=\left(x,\left(\theta_{1}-\theta_{0}\right) y\right)$ for $x, y \in X_{0} \cap X_{1}$, which is dense in $d X_{\eta}$.

To see that $T$ is well-defined take $f \in \mathscr{F}_{0}(\bar{X})$ such that $f(\eta)=x, f^{\prime}(\eta)=y$. Then $g$ given by $g(z)=f\left((1-z) \theta_{0}+z \theta_{1}\right)$ is in $\mathscr{F}(\bar{Y}), g(\theta)=x$ and $g^{\prime}(\theta)=\left(\theta_{1}-\theta_{0}\right) y$. Therefore, $T$ is well-defined.

Also, $\|g\|_{\mathscr{F}(\bar{Y})} \leq\|f\|_{\mathscr{F}(\bar{X})}$, and $T$ is continuous and may be extended to all of $d X_{\eta}$. Since this may be done in $M_{n}(\mathscr{F}(\bar{Y}))$, we get that $T$ is completely bounded. 


\subsection{Complex interpolation of operator spaces through $L_{p^{-}}$ norms}

Recall from Section 2.5 that complex interpolation of couples of Banach spaces may be done through a variation of the space $\mathscr{F}$. We show now how these ideas extend to the operator space case.

Definition 5.6.1. Let $(M, \mu)$ be a measure space, $X$ a Banach space and $p \in[1, \infty)$. If $\alpha>0$, we let $\alpha L_{p}(\mu, X)$ be the Banach (operator) space $\left(L_{p}(\mu), \alpha^{\frac{1}{p}}\|\cdot\|_{L_{p}(\mu, X)}\right)$.

Recall the definition of the space $\mathscr{F}_{\theta}^{p}$ from 2.5.1.

Definition 5.6.2. Let $\bar{X}=\left(X_{0}, X_{1}\right)$ be a compatible couple of operator spaces, $0<\theta<1$, $p \in[1, \infty)$. The o. s. s. on the space $\mathscr{F}_{p}^{\theta}$ is the one induced by the inclusion

$$
\mathscr{F}_{p}^{\theta} \subset(1-\theta) L_{p}\left(\mathbb{S}_{0}, \mu_{\theta}^{0}, X_{0}\right) \oplus_{p} \theta L_{p}\left(\mathbb{S}_{1}, \mu_{\theta}^{1}, X_{1}\right)
$$

The following result is due to Pisier and Qiu, and was first presented in (CORREAA, 2018a).

Theorem 5.6.3. [G. Pisier, Y. Qiu] Let $\bar{X}=\left(X_{0}, X_{1}\right)$ be a compatible couple of operator spaces, $0<\theta<1$ and $p \in[1, \infty)$. Then we have $X_{\theta}=\mathscr{F}_{p}^{\theta} / \operatorname{ker}\left(\delta_{\theta}\right)$ completely isometrically.

Proof. Recall from Proposition 4.9.3 that we have isometrically for every $n \in \mathbb{N}$

$$
\left(S_{p}^{n}\left[X_{0}\right], S_{p}^{n}\left[X_{1}\right]\right)_{\theta}=S_{p}^{n}\left[X_{\theta}\right]
$$

By the Banach space version of the theorem we wish to prove, we have for every $x \in S_{p}^{n}\left[X_{\theta}\right]$

$$
\|x\|_{S_{p}^{n}\left[X_{\theta}\right]}^{p}=\inf \left\{(1-\theta)\left\|\left.f\right|_{\mathbb{S}_{0}}\right\|_{L_{p}\left(\mathbb{S}_{0}, \mu_{\theta}^{0}, S_{p}^{n}\left[X_{0}\right]\right)}^{p}+\theta\left\|\left.f\right|_{\mathbb{S}_{1}}\right\|_{L_{p}\left(\mathbb{S}_{1}, \mu_{\theta}^{1}, S_{p}^{n}\left[X_{1}\right]\right)}^{p}\right\}
$$

where the infimum is over all $f \in \mathscr{F}_{p}^{\theta}\left(S_{p}^{n}\left[X_{0}\right], S_{p}^{n}\left[X_{1}\right]\right)$ such that $f(\theta)=x$. By Proposition 4.9.4

$$
\|x\|_{S_{p}^{n}\left[X_{\theta}\right]}^{p}=\inf \left\{(1-\theta)\left\|\left.f\right|_{\mathbb{S}_{0}}\right\|_{S_{p}^{n}\left[L_{p}\left(\mathbb{S}_{0}, \mu_{\theta}^{0}, X_{0}\right)\right]}^{p}+\theta\left\|\left.f\right|_{\mathbb{S}_{1}}\right\|_{S_{p}^{n}\left[L_{p}\left(\mathbb{S}_{1}, \mu_{\theta}^{1}, X_{1}\right)\right]}^{p}\right\}
$$

By Proposition 4.9.5

$$
\|x\|_{S_{p}^{n}\left[X_{\theta}\right]}^{p}=\inf \left\{\|f\|_{S_{p}^{n}\left[\mathscr{F}_{p}^{\theta}\right]}^{p}: f(\theta)=x\right\}
$$

The theorem now follows from Proposition 4.9.2.

Lemma 5.6.4. Let $\bar{X}=\left(X_{0}, X_{1}\right)$ be a compatible couple of operator spaces, $\theta \in(0,1)$, and let $\varphi: \mathbb{S} \rightarrow \mathbb{D}$ be a conformal equivalence with $\varphi(\theta)=0$. Let $p \in[1, \infty)$ and $T_{\varphi}: \mathscr{F}_{p}^{\theta} \rightarrow \operatorname{ker}\left(\delta_{\theta}\right) \subset$ $\mathscr{F}_{p}^{\theta}$ be given by $T_{\varphi}(f)=\varphi f$. Then $T_{\varphi}$ is a complety isometry.

Proof. Let $f \in \mathscr{F}_{p}^{\theta}$.

We begin by showing that $T_{\varphi}(f) \in \mathscr{F}_{p}^{\theta}$.

Properties 1., 2. and 4. from Definition 2.5.1 are clear. Let us show 3.

Let $x^{*} \in \Sigma(\bar{X})^{*}$ and define $g: \mathbb{D} \rightarrow \mathbb{S}$ by

$$
g(z)=x^{*}\left(z f\left(\varphi^{-1}(z)\right)\right)
$$


For every $z \in \mathbb{D}$ we have

$$
|g(z)| \leq\left\|x^{*}\right\|\|f\|
$$

so that $g \in H^{\infty}$. Therefore, $g$ satisfies the relation

$$
g\left(\varphi\left(z_{0}\right)\right)=\int_{\mathbb{T}} g(z) P_{\varphi\left(z_{0}\right)}(z) d z
$$

so that

$$
x^{*}\left(\varphi\left(z_{0}\right) f\left(z_{0}\right)\right)=x^{*} \int_{\mathbb{T}} z f\left(\varphi^{-1}(z)\right) P_{\varphi\left(z_{0}\right)}(z) d z=x^{*} \int_{\partial \mathbb{S}} \varphi(z) f(z) d \mu_{z_{0}}(z)
$$

Since $x^{*}$ and $z_{0}$ were arbitrary, we obtain 3., and $\varphi f \in \mathscr{F}_{p}^{\theta}$.

It is also clear that $f \in \operatorname{ker}\left(\delta_{\theta}\right)$ and that $\|\varphi f\|_{p}=\|f\|$, so that $T_{\varphi}$ is an isometry onto its image. Using the spaces $S_{p}^{n}$, with the same argument from Theorem 5.6.3 we prove that $T_{\varphi}$ is a complete isometry onto its image. All that remains to be proved is that $\operatorname{ker}\left(\delta_{\theta}\right) \subset \operatorname{Im}\left(T_{\varphi}\right)$.

Let $f \in \operatorname{ker}\left(\delta_{\theta}\right) \subset \mathscr{F}_{p}^{\theta}$ and $x^{*} \in \Sigma(\bar{X})$. Define $g: \mathbb{D} \rightarrow \mathbb{C}$ by

$$
g(z)=x^{*}\left(f\left(\varphi^{-1}(z)\right)\right)
$$

Then again $g \in H^{\infty}$, so that $g \in N^{+}$. Also, since $g(0)=0$, we have by Lemma 2.3.61 that $\frac{g}{z} \in N^{+}$, and by Proposition 2.3.50 this implies that $\frac{g}{z} \in H^{\infty}$.

As above, this implies that $\frac{f}{\varphi}$ satisfies 3. from Definition 2.5.1, the others properties being clear, so that $\frac{f}{\varphi} \in \mathscr{F}_{p}^{\theta}$ and $T_{\varphi}\left(\frac{f}{\varphi}\right)=f$, and the result is proved.

We may now define a variation of the operator space $d X_{\theta}$ and prove that it is an equivalent complete extension of $X_{\theta}$.

Definition 5.6.5. Let $\bar{X}=\left(X_{0}, X_{1}\right)$ be a compatible couple of operator spaces, $\theta \in(0,1)$, and $p \in[1, \infty)$. Let $d X_{\theta}^{p}=\mathscr{F}_{p}^{\theta} /\left(\operatorname{ker}\left(\delta_{\theta}\right) \cap \operatorname{ker}\left(\delta_{\theta}^{\prime}\right)\right)$.

Proposition 5.6.6. Let $\bar{X}=\left(X_{0}, X_{1}\right)$ be a compatible couple of operator spaces, $\theta \in(0,1)$, and $p \in[1, \infty)$. Then we have an extension sequence

$$
0 \longrightarrow X_{\theta} \stackrel{i^{p}}{\longrightarrow} d X_{\theta}^{p} \stackrel{q^{p}}{\longrightarrow} X_{\theta} \longrightarrow 0
$$

where $i^{p}(y)=(0, y)$ and $q^{p}(x, y)=x$.

Proof. The proof follows the lines of Proposition 5.5.6.

It is clear that $i^{p}$ is injective and that $q^{p}$ is surjective.

Let $\varphi: \mathbb{S} \rightarrow \mathbb{D}$ be a conformal map with $\varphi(\theta)=0$.

1. $\operatorname{ker}\left(q^{p}\right)=\operatorname{Im}\left(i^{p}\right)$

We have an obvious inclusion $\operatorname{Im}\left(i^{p}\right) \subset \operatorname{ker}\left(q^{p}\right)$. Suppose that $(0, y) \in \operatorname{ker}\left(q^{p}\right)$. Then there is $f \in \mathscr{F}_{p}^{\theta}$ such that $f(\theta)=0$ and $f^{\prime}(\theta)=y$. By Lemma 5.6.4, the function $g=\frac{f}{\varphi}$ is in $\mathscr{F}_{p}^{\theta}$.

Since $g(\theta)=\frac{y}{\left|\varphi^{\prime}(\theta)\right|}$, we have that $y \in X_{\theta}$ and $(0, y)=i^{p}(y)$.

2. $i^{p}$ is a complete isomorphism onto its image

Let $y \in M_{n}\left(X_{\theta}\right)$, and take $f \in M_{n}\left(\mathscr{F}_{p}^{\theta}\right)$ such that $f(\theta)=y$. By Lemma 5.6.4, the function $g=\varphi f$ is in $M_{n}\left(\mathscr{F}_{p}^{\theta}\right)$. Furthermore, $\|g\|=\|f\|, g(\theta)=0$ and $g^{\prime}(\theta)=\varphi^{\prime}(\theta) y$. Therefore

$$
\|(0, y)\| \leq\left|\varphi^{\prime}(\theta)\right|\|g\|=\left|\varphi^{\prime}(\theta)\right|\|f\|
$$


Since $f$ was arbitrary, we have that $\|(0, y)\| \leq\left|\varphi^{\prime}(\theta)\right|\|y\|$, and $i^{p}$ is completely bounded.

To check that $i^{p}$ is a complete isomorphism onto its image, given $y \in M_{n}\left(X_{\theta}\right)$ and $g \in M_{n}(\underset{F}{\theta})$ such that $g(\theta)=0$ and $g^{\prime}(\theta)=y$ we have again by Lemma 5.6.4 that the function $f=\frac{g}{\varphi}$ is in $M_{n}\left(\mathscr{F}_{p}^{\theta}\right)$. Furthermore, $\|f\|=\|g\|$ and $f(\theta)=\frac{y}{\left|\varphi^{\prime}(\theta)\right|}$, so that

$$
\|y\| \leq\left|\varphi^{\prime}(\theta)\right|\|f\|=\left|\varphi^{\prime}(\theta)\right|\|g\|
$$

Since $g$ was arbitrary we obtain $\|y\| \leq\left|\varphi^{\prime}(\theta)\right|\|(0, y)\|$. So $i^{p}$ is a complete isomorphism onto its image (actually, it is a multiple of a complete isometry).

3. $q^{p}$ is completely bounded

Given $(x, y) \in M_{n}\left(d X_{\theta}^{p}\right)$ we have

$$
\begin{aligned}
\left\|\left(q^{p}\right)_{n}(x, y)\right\| & =\|x\| \\
& =\inf \left\{\|f\|: f \in \mathscr{F}_{p}^{\theta}, f(\theta)=x\right\} \\
& \leq \inf \left\{\|f\|: f \in \mathscr{F}_{p}^{\theta}, f(\theta)=x, f^{\prime}(\theta)=y\right\} \\
& =\|(x, y)\|
\end{aligned}
$$

so that $\left\|q^{p}\right\|_{c b} \leq 1$.

\section{4. $q^{p}$ is a complete quotient map}

Since $q^{p}$ is c. b. we have that $\tilde{q}^{p}$ is c. b. Let $(x, y) \in M_{n}\left(d X_{\theta}^{p}\right)$ and take $f \in M_{n}\left(\mathscr{F}_{p}^{\theta}\right)$ such that $f(\theta)=x$ and $f^{\prime}(\theta)=y$. Then

$$
\begin{aligned}
\left\|\tilde{q^{p}}\left((x, y)+M_{n}\left(i^{p}\left(X_{\theta}\right)\right)\right)\right\| & =\|x\| \\
& =\inf \left\{\|f+g\|: g \in M_{n}\left(\operatorname{ker}\left(\delta_{\theta}\right)\right)\right\} \\
& =\inf \left\{\|h\|: h(\theta)=x, h^{\prime}(\theta)-f^{\prime}(\theta) \in M_{n}\left(X_{\theta}\right)\right\} \\
& =\inf \left\{\|h\|: h(\theta)=x, h^{\prime}(\theta) \in y+M_{n}\left(X_{\theta}\right)\right\} \\
& =\left\|(x, y)+M_{n}\left(i^{p}\left(X_{\theta}\right)\right)\right\|
\end{aligned}
$$

Therefore, $q^{p}$ induces a complete isometry between $d X_{\theta}^{p} / i^{p}\left(X_{\theta}\right)$ and $X_{\theta}$.

Lemma 5.6.7. Let $\bar{X}=\left(X_{0}, X_{1}\right)$ be a compatible couple of operator spaces, $\theta \in(0,1)$, and $p \in[1, \infty)$. Then we have a c. b. inclusion $\mathscr{F} \subset \mathscr{F}_{\theta}^{p}$.

Proof. We begin by showing that if $f \in \mathscr{F}$ then $f \in \mathscr{F}_{p}^{\theta}$.

1., 2. and 4. from Definition 2.5.1 are clear. To show 3., let $x^{*} \in \Sigma(\bar{X}), \varphi: \mathbb{S} \rightarrow \mathbb{D}$ be a conformal map and $g: \mathbb{D} \rightarrow \mathbb{C}$ be given by

$$
g(z)=x^{*}\left(f\left(\varphi^{-1}(z)\right)\right)
$$

Then $g \in H^{\infty}$ and therefore, for every $z_{0} \in \mathbb{S}$, we have

$$
\begin{aligned}
g\left(\varphi\left(z_{0}\right)\right) & =\int_{\mathbb{T}} g(z) P_{\varphi\left(z_{0}\right)}(z) d z \\
& =x^{*} \int_{\mathbb{T}} f\left(\varphi^{-1}(z)\right) P_{\varphi\left(z_{0}\right)}(z) d z \\
& =x^{*} \int_{\partial \mathbb{S}} f(z) d \mu_{z_{0}}(z)
\end{aligned}
$$

Since $g\left(\varphi\left(z_{0}\right)\right)=x^{*} f\left(z_{0}\right)$, and $x^{*}$ was arbitrary we obtain 3 ., and $f \in \mathscr{F}_{p}^{\theta}$. 
To see that the inclusion $\mathscr{F} \subset \mathscr{F}_{p}^{\theta}$ is completely bounded notice that the o. s. s. on $\mathscr{F}$ is induced by the inclusion

$$
\mathscr{F} \subset L_{\infty}\left(\mathbb{S}_{0}, \mu_{\theta}^{0}, X_{0}\right) \oplus_{\infty} L_{\infty}\left(\mathbb{S}_{1}, \mu_{\theta}^{1}, X_{1}\right)
$$

But we have c. b. norm 1 inclusions $L_{\infty}\left(\mathbb{S}_{j}, \mu_{\theta}^{j}, X_{j}\right) \subset L_{p}\left(\mathbb{S}_{j}, \mu_{\theta}^{j}, X_{j}\right), j=0,1$. Also, for any couple of Banach spaces $X$ and $Y$ we have a c. b. inclusion $X \oplus_{\infty} Y \subset X \oplus_{p} Y$, so that we have a chain of c. b. inclusions

$$
\begin{aligned}
L_{\infty}\left(\mathbb{S}_{0}, \mu_{\theta}^{0}, X_{0}\right) \oplus_{\infty} L_{\infty}\left(\mathbb{S}_{1}, \mu_{\theta}^{1}, X_{1}\right) & \subset L_{\infty}\left(\mathbb{S}_{0}, \mu_{\theta}^{0}, X_{0}\right) \oplus_{p} L_{\infty}\left(\mathbb{S}_{1}, \mu_{\theta}^{1}, X_{1}\right) \\
& \subset L_{p}\left(\mathbb{S}_{0}, \mu_{\theta}^{0}, X_{0}\right) \oplus_{p} L_{p}\left(\mathbb{S}_{1}, \mu_{\theta}^{1}, X_{1}\right) \\
& \subset(1-\theta) L_{p}\left(\mathbb{S}_{0}, \mu_{\theta}^{0}, X_{0}\right) \oplus_{p} \theta L_{p}\left(\mathbb{S}_{1}, \mu_{\theta}^{1}, X_{1}\right)
\end{aligned}
$$

and this implies that the inclusion $\mathscr{F} \subset \mathscr{F}_{p}^{\theta}$ is completely bounded.

Proposition 5.6.8. Let $\bar{X}=\left(X_{0}, X_{1}\right)$ be a compatible couple of operator spaces, $\theta \in(0,1)$, and $p \in[1, \infty)$. Then we have a complete equivalence of extension sequences

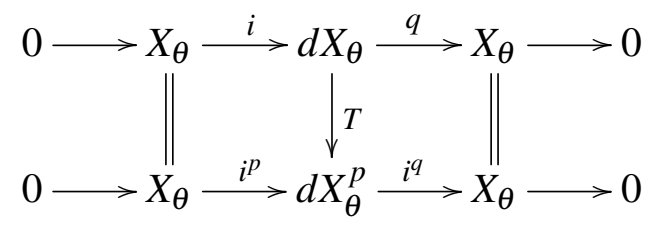

where $T(x, y)=(x, y)$.

Proof. Let $(x, y) \in M_{n}\left(d X_{\theta}\right)$. Then, by Lemma 5.6.7, there is a constant $C>0$ independent of $n$ such that

$$
\begin{aligned}
\|(x, y)\|_{M_{n}\left(d X_{\theta}^{p}\right)} & =\inf \left\{\|f\|_{p}: f \in M_{n}\left(\mathscr{F}_{p}^{\theta}\right), f(\theta)=x, f^{\prime}(\theta)=y\right\} \\
& \leq C \inf \left\{\|f\|_{\infty}: f \in M_{n}\left(\mathscr{F}_{p}^{\theta}\right), f(\theta)=x, f^{\prime}(\theta)=y\right\} \\
& \leq C \inf \left\{\|f\|_{\infty}: f \in M_{n}(\mathscr{F}), f(\theta)=x, f^{\prime}(\theta)=y\right\} \\
& =C\|(x, y)\|_{M_{n}\left(d X_{\theta}\right)}
\end{aligned}
$$

so that $T$ is completely bounded. Since $T$ makes the diagram commute, an application of the 3-Lemma 5.1.10 gives that the extension sequences are equivalent.

\subsubsection{Duality}

We now treat the duality of twisted sums induced by complex interpolation. This result will be useful to prove complete cosingularity.

Again, to talk about duality we specialize to the case of complex interpolation of couples. Recall that to prove duality of twisted sums induced by complex interpolation (Theorem 3.3.12) we used the Schwarz-Pick Lemma 3.3.9. To prove the operator space version of Theorem 3.3.12 we shall use a noncommutative version of the lemma.

We point out that there are more informative versions of the Schwarz-Pick Lemma in the context of analytic functions with image in a space of operators (ANDERSON; ROVNYAK, 2006), but the following is enough for our needs. 
Lemma 5.6.9. [Noncommutative Schwarz-Pick Lemma] Let $F: \mathbb{D} \rightarrow M_{n}$ be an analytic function such that $\|F(z)\| \leq 1$ for every $z \in \mathbb{D}$. Then

$$
\left\|F^{\prime}(z)\right\| \leq \frac{1}{1-|z|^{2}}
$$

for every $z \in \mathbb{D}$.

Proof. For $x, y \in \ell_{2}^{n}$ of norm 1 let $H_{x, y}: \mathbb{D} \rightarrow \mathbb{C}$ be given by

$$
H_{x, y}(z)=\langle F(z) x, y\rangle
$$

Then $H_{x, y}$ is an analytic function with image in $\mathbb{D}$ and its derivative is given by

$$
H_{x, y}(z)=\left\langle F^{\prime}(z) x, y\right\rangle
$$

By the Schwarz-Pick Lemma 3.3.9, we have

$$
\left|\left\langle F^{\prime}(z) x, y\right\rangle\right|=\left|H_{x, y}^{\prime}(z)\right| \leq \frac{1}{1-|z|^{2}}
$$

Since $x$ and $y$ are arbitrary of norm 1 we obtain

$$
\left\|F^{\prime}(z)\right\| \leq \frac{1}{1-|z|^{2}}
$$

Lemma 5.6.10. Let $H: \overline{\mathbb{S}} \rightarrow M_{n}$ be a bounded continuous function, analytic on $\mathbb{S}$. Then for every $z \in \overline{\mathbb{S}}$ we have

$$
\|H(z)\| \leq \sup \{\|H(w)\|: w \in \partial \mathbb{S}\}
$$

Proof. Let $x, y \in \ell_{2}$ have norm 1 and let $H_{x, y}: \overline{\mathbb{S}} \rightarrow \mathbb{C}$ be given by

$$
H_{x, y}(z)=\langle H(z) x, y\rangle
$$

Then $H_{x, y}$ is continuous, bounded, and it is also analytic on $\mathbb{S}$. Therefore, for every $z \in \overline{\mathbb{S}}$ we have

$$
\left|H_{x, y}(z)\right| \leq \sup \left\{\left\|H_{x, y}(w)\right\|: w \in \partial \mathbb{S}\right\} \leq \sup \{\|H(w)\|: w \in \partial \mathbb{S}\}
$$

Since $x$ and $y$ were arbitrary of norm 1 we obtain

$$
\|H(z)\| \leq \sup \{\|H(w)\|: w \in \partial \mathbb{S}\}
$$

Theorem 5.6.11. Let $\bar{X}=\left(X_{0}, X_{1}\right)$ be a compatible couple of operator spaces such that $\Delta(\bar{X})$ is dense in $X_{0}$ and $X_{1}$ and such that at least one of the spaces $X_{0}$ or $X_{1}$ is reflexive. Then the extension sequences

$$
0 \longrightarrow X_{\theta}^{*} \longrightarrow d\left(X_{\theta}^{*}\right) \longrightarrow X_{\theta}^{*} \longrightarrow 0
$$

and

$$
0 \longrightarrow X_{\theta}^{*} \longrightarrow\left(d X_{\theta}\right)^{*} \longrightarrow X_{\theta}^{*} \longrightarrow 0
$$

are completely (isomorphically) equivalent. 
Proof. We will prove that the operator $T: d\left(X_{\theta}^{*}\right) \rightarrow\left(d X_{\theta}^{*}\right)$ from Theorem 3.3.12 is completely bounded. We begin by recalling the definition of $T$.

Let

$$
A=\left\{\left(x^{*}, y^{*}\right) \in d\left(X_{\theta}^{*}\right): x^{*}, y^{*} \in \Delta\left(\overline{X^{*}}\right)\right\}
$$

and

$$
B=\left\{(x, y) \in d X_{\theta}: x, y \in \Delta(\bar{X})\right\}
$$

Then by Lemma 3.3.6 $A$ is dense in $d\left(X_{\theta}^{*}\right)$ and $B$ is dense in $d X_{\theta}$.

For $\left(x^{*}, y^{*}\right) \in B$ and $(x, y) \in A$ the map $T$ is defined as

$$
T\left(x^{*}, y^{*}\right)(x, y)=\left\langle x^{*}, y\right\rangle+\left\langle y^{*}, x\right\rangle
$$

It was proved in Theorem 3.3.12 that $T$ defines an isomorphism. Let us see that it is completely bounded.

We will use the isometric identity $M_{n}\left(\left(d X_{\theta}^{*}\right)\right)=C B\left(d X_{\theta}, M_{n}\right)$.

Let $\varepsilon>0$. Given $\left(x^{*}, y^{*}\right) \in M_{n}(A)$ and $(x, y) \in M_{m}(B)$ take $f \in M_{n}\left(\mathscr{F}_{0}\left(\overline{X^{*}}\right)\right)$ and $g \in$ $M_{m}\left(\mathscr{F}_{0}(\bar{X})\right)$ such that

- $f(\boldsymbol{\theta})=x^{*}, f^{\prime}(\boldsymbol{\theta})=y^{*}$;

- $g(\theta)=x, g^{\prime}(\theta)=y$;

- $\|f\| \leq\left\|\left(x^{*}, y^{*}\right)\right\|+\varepsilon$

- $\|g\| \leq\|(x, y)\|+\varepsilon$.

We may do that by Lemma 3.3.6.

Let $H: \bar{S} \rightarrow M_{m n}$ be given by

$$
H(z)=\left\langle(f(z))_{m}, g(z)\right\rangle
$$

By our choice of $f$ and $g$ we have that $H$ is analytic and bounded (since each entry is bounded). We also have for $j=0,1$ and $t \in \mathbb{R}$

$$
\begin{aligned}
\|H(j+i t)\| & =\left\|\left\langle(f(j+i t))_{m}, g(j+i t)\right\rangle\right\| \\
& \leq\|f(j+i t)\|_{C B\left(X_{j}, M_{n}\right)}\|g(j+i t)\|_{M_{m}\left(X_{j}\right)} \\
& =\|f(j+i t)\|_{M_{n}\left(X_{j}^{*}\right)}\|g(j+i t)\|_{M_{m}\left(X_{j}\right)} \\
& \leq\|f\|\|g\|
\end{aligned}
$$

Let $\varphi: \mathbb{D} \rightarrow \mathbb{S}$ be a conformal map with $\varphi(0)=\theta$. Then, by Lemma 5.6.10, the map $h: \mathbb{D} \rightarrow M_{n}$ given by

$$
h=\frac{H \circ \varphi}{2\|f\|\|g\|}
$$

is such that $\|h(z)\|<1$ for every $z \in \mathbb{D}$. By Lemma 5.6.9

$$
\left\|h^{\prime}(0)\right\| \leq 1
$$

But

$$
h^{\prime}(0)=\frac{\varphi^{\prime}(0)}{2\|f\|\|g\|} H^{\prime}(\theta)
$$

So

$$
\left\|H^{\prime}(\theta)\right\| \leq \frac{2\|f\|\|g\|}{\left|\varphi^{\prime}(0)\right|}
$$


But

$$
H^{\prime}(\theta)=\left(T_{n}\left(x^{*}, y^{*}\right)\right)_{m}(x, y)
$$

so that

$$
\left\|\left(T_{n}\left(x^{*}, y^{*}\right)\right)_{m}(x, y)\right\| \leq \frac{2}{\left|\varphi^{\prime}(0)\right|}\left(\left\|\left(x^{*}, y^{*}\right)\right\|+\varepsilon\right)(\|(x, y)\|+\varepsilon)
$$

Since $\varepsilon$ and $m$ were arbitrary and by the density of $B$ we obtain

$$
\left\|T_{n}\left(x^{*}, y^{*}\right)\right\|_{c b} \leq \frac{2}{\left|\varphi^{\prime}(0)\right|}\left\|\left(x^{*}, y^{*}\right)\right\|
$$

By the density of $A$ and since $n$ was arbitrary we obtain in turn

$$
\|T\|_{c b} \leq \frac{2}{\left|\varphi^{\prime}(0)\right|}
$$

So that $T$ is c. b., and by the 3-lemma 5.1.10 it is a complete isomorphism.

\subsection{Complete Exact Sequences and the Haagerup Tensor Product}

We postponed until now the discussion of the projectivity of the Haagerup tensor product.

Recall from Section 4.5 that the Haagerup tensor product is injective. One of its important properties is that it is also projective. The following is a straightforward modification of Proposition 9.2.5 of (EFFROS; RUAN, 2000), which states the result for complete quotient maps $q$ such that $\tilde{q}$ is a complete isometry.

Proposition 5.7.1. Let $X, X^{\prime}, Y, Y^{\prime}$ be operator spaces and $\varphi: X \rightarrow X^{\prime}, \psi: Y \rightarrow Y^{\prime}$ be complete quotient maps. Then

$$
\varphi \otimes \psi: X \otimes \otimes^{h} Y \rightarrow X^{\prime} \otimes^{h} Y^{\prime}
$$

is a complete quotient map.

So, given a complete twisted sum

$$
0 \longrightarrow Y \stackrel{i}{\longrightarrow} X \stackrel{q}{\longrightarrow} Z \longrightarrow 0
$$

and an operator space $X^{\prime}$, we get two new short sequences:

$$
0 \longrightarrow Y \otimes^{h} X^{\prime} \stackrel{i \otimes I d_{X^{\prime}}}{\longrightarrow} X \otimes^{h} X^{\prime} \stackrel{q \otimes I d_{X^{\prime}}}{\longrightarrow} Z \otimes^{h} X^{\prime} \longrightarrow 0
$$

and

$$
0 \longrightarrow X^{\prime} \otimes^{h} Y \stackrel{I d_{X^{\prime}} \otimes i}{\longrightarrow} X^{\prime} \otimes^{h} X \stackrel{I d_{X^{\prime}} \otimes q}{\longrightarrow} X^{\prime} \otimes^{h} Z \longrightarrow 0
$$

(the Haagerup tensor product is not commutative).

Proposition 5.7.2. The sequences (5.8) and (5.9) are extension sequences.

Proof. Exactness is a simple adaptation of Proposition 7.1.7 of (EFFROS; RUAN, 2000). The result follows from the fact that the Haagerup tensor product is both injective and projective.

Proposition 5.7.3. The extension sequence (5.7) is completely trivial if and only if (5.8) ((5.9)) is completely trivial. 
Proof. The only if part is an application of Proposition 5.1.14.

We prove the if part only for (5.9). Let $V$ be a one-dimensional subspace of $X^{\prime}$ and let $P: X^{\prime} \rightarrow V$ be a completely bounded projection. We identify $V$ with $\mathbb{C}$ naturally.

Let $T_{X}: \mathbb{C} \otimes^{h} X \rightarrow X$ be the natural complete isometry, and $j: \mathbb{C} \otimes^{h} Z \rightarrow X^{\prime} \otimes^{h} Z$ the natural completely isometric inclusion.

Let $s: X^{\prime} \otimes^{h} Z \rightarrow X^{\prime} \otimes^{h} Z$ be such that $\left(I d_{X^{\prime}} \otimes q\right) \circ s=I d_{X^{\prime}} \otimes^{h} Z$.

Notice that

$$
\begin{aligned}
q \circ T_{X}\left(\sum \alpha_{i} \otimes x_{i}\right) & =q\left(\sum \alpha_{i} x_{i}\right) \\
& =\sum \alpha_{i} q\left(x_{i}\right) \\
& =T_{Z}\left(\sum \alpha_{i} \otimes q\left(x_{i}\right)\right) \\
& =T_{Z} \circ\left(I d_{\mathbb{C}} \otimes q\right)\left(\sum \alpha_{i} \otimes x_{i}\right)
\end{aligned}
$$

and:

Let $s^{\prime}: Z \rightarrow X$ be given by $s^{\prime}=T_{X} \circ\left(P \otimes I d_{X}\right) \circ s \circ j \circ T_{Z}^{-1}$. Then $s^{\prime}$ is completely bounded

$$
\begin{aligned}
q \circ s^{\prime}(z) & =q \circ T_{X} \circ\left(P \otimes I d_{X}\right) \circ s \circ j \circ T_{Z}^{-1}(z) \\
& =T_{Z} \circ\left(I d_{\mathbb{C}} \otimes q\right) \circ\left(P \otimes I d_{X}\right) \circ s(1 \otimes z) \\
& =T_{Z} \circ\left(P \otimes I d_{Z}\right) \circ\left(I d_{\mathbb{C}} \otimes q\right) \circ s(1 \otimes z) \\
& =T_{Z} \circ\left(P \otimes I d_{Z}\right)(1 \otimes z) \\
& =z
\end{aligned}
$$

Therefore, by Proposition 5.1.14, the complete twisted sum (5.7) is completely trivial.

Notice that we did not use in the proof any special property of the Haagerup tensor product, so it is valid for any reasonable tensor product whenever sequences (5.8) and 5.9 are well-defined substituting the Haagerup tensor product. So we have another proof of Proposition 5.2.1. By reasonable we mean that

- for any operator space $X$ the tensor product $\mathbb{C} \otimes X$ is naturally completely isometric to $X$;

- if $X, Y$ and $Z$ are operator spaces and $T: X \rightarrow Y$ is a c. b. map with finite range then $\left\|T \otimes I d_{Z}\right\|_{c b}=\|T\|_{c b}$.

As in Banach space theory we can consider injective and projective objects in the category of operator spaces.

Definition 5.7.4. An operator space $Y$ (respectively, $Z$ ) is called injective (respectively, projective) if every extension sequence

$$
0 \longrightarrow Y \longrightarrow X \longrightarrow Z \longrightarrow 0
$$

is completely trivial.

Observation 5.7.5. In (RUAN, 1989) an apparently more restrictive definition for injective operator spaces is given: $Y$ is injective if $X_{0} \subset X$ are operator spaces and $T_{0}: X_{0} \rightarrow Y$ is completely bounded then there is an extension $T: X \rightarrow Y$ of $T_{0}$ such that $\|T\|_{c b}=\left\|T_{0}\right\|_{c b}$.

In (RUAN, 1989) it is also proved the existence of injective envelopes in the category of operator spaces, i. e., given an operator space $Y$ there is an injective operator space $X$ and a 
completely isometric embedding $i: Y \rightarrow X$ such that if $T: X \rightarrow X$ is such that $\left.T\right|_{i(Y)}=I d_{i(Y)}$ then $T=I d_{X}$.

If $Y$ is injective according to our definition, taking an injective envelope $(X, i)$ of $Y$ we may consider the extension sequence

$$
0 \longrightarrow Y \stackrel{i}{\longrightarrow} X \longrightarrow X / Z \longrightarrow 0
$$

which is completely trivial. Therefore, there is a completely bounded retraction $r: X \rightarrow Y$ for $i$, that is, $r \circ i=I d_{Y}$. If we let $T=i \circ r: X \rightarrow X$, then $\left.T\right|_{i(Y)}=I d_{i(Y)}$, and therefore $i \circ r=I d_{X}$, i. e., $Y$ is completely isomorphic to $X$.

This shows that an operator space $Y$ is injective according to our definition if and only if it is completely isomorphic to an injective operator space according to the definition of (RUAN, 1989). Also, our definition is equivalent to the one of injective operator space of (PISIER, 2003).

Examples of injective operator spaces are $R$ and $C$.

Observation 5.7.6. As for injective operator spaces, the original definition for projectivity is more restrictive: in (BLECHER, 1992) an operator space $Z$ is called projective if for any $\varepsilon>0$, any operator space $Y \subset X$ and any completely contractive $T: Z \rightarrow X / Y$ there is a lifting $\tilde{T}: Z \rightarrow X$ of $T$ such that $\|\tilde{T}\|_{c b} \leq 1+\varepsilon$.

Examples of projective operator spaces are the spaces

$$
\ell_{1}\left(\left\{S_{1}^{n_{i}}: i \in I\right\}\right)
$$

Any operator space may be written as a quotient of an operator space of this form ((PISIER, 2003), 2.12.2). So if $Z$ is an operator space which is projective according to our definition and we take an extension sequence

$$
0 \longrightarrow Y \stackrel{i}{\longrightarrow} X \stackrel{q}{\longrightarrow} Z \longrightarrow 0
$$

where $X$ is like in (5.10) then we have the isomorphism $\tilde{q}: X / Y \rightarrow Z$. Since the extension sequence completely splits, there is a c. b. section $s: Z \rightarrow X$ for $q$, that is, $q \circ s=I d_{Z}$.

According to (PISIER, 2003), Proposition 24.5, this means that there is $\lambda>0$ such that for any $\varepsilon>0$, any operator space $Y_{0} \subset X_{0}$ and any completely contractive $T: Z \rightarrow X_{0} / Y_{0}$ there is a lifting $\tilde{T}: Z \rightarrow X_{0}$ of $T$ such that $\|\tilde{T}\|_{c b} \leq \lambda+\varepsilon$.

That is, our definition is equivalent to the definition of $\lambda$-projectivity of (PISIER, 2003).

As a corollary to Proposition 5.7.3 we obtain:

Corollary 5.7.7. Suppose that $X_{1} \otimes{ }^{h} X_{2}$ is injective (projective). Then $X_{1}$ and $X_{2}$ are injective (projective).

Proof. Suppose that $X_{1}$ is not injective. Then there is an extension sequence which is not completely trivial

$$
0 \longrightarrow X_{1} \longrightarrow X \longrightarrow Z \longrightarrow 0
$$

Tensoring with $X_{2}$ we get an extension sequence

$$
0 \longrightarrow X_{1} \otimes^{h} X_{2} \longrightarrow X \otimes^{h} X_{2} \longrightarrow Z \otimes^{h} X_{2} \longrightarrow 0
$$

which is not completely trivial, and therefore $X_{1} \otimes{ }^{h} X_{2}$ is not injective. The rest of the proof is similar. 
Observation 5.7.8. Given a complete twisted sum (5.7), and an infinite dimensional operator space $X^{\prime}$, the tensored complete twisted sums (5.8) and (5.9) are never completely singular.

To see that, take $\mathbb{C} \subset Z$. Then $X^{\prime}=\mathbb{C} \otimes{ }^{h} X^{\prime} \subset Z \otimes^{h} X^{\prime}$ is infinite dimensional. Let $s: \mathbb{C} \rightarrow q^{-1}(\mathbb{C})$ be a completely bounded section for $\left.q\right|_{q^{-1}(\mathbb{C})}$. Then $s \otimes I d_{X^{\prime}}$ is a completely bounded section for $\left.\left(q \otimes I d_{X^{\prime}}\right)\right|_{\left(q \otimes I d_{X^{\prime}}\right)^{-1}\left(\mathbb{C} \otimes h X^{\prime}\right)}$. 



\section{CONCRETE TWISTED SUMS OF OPERATOR SPACES}

In this chapter we apply the results from Chapter 5 to obtain concrete examples of complete twisted sums. In particular, we are interested in solving the following problems:

Palais' Problem for Operator Spaces (Isomorphic Version). Let $X$ be a $\lambda$-Hilbertian operator space for some $\lambda \geq 1$ and suppose that $X$ is a complete extension of $O H$. Is $X$ completely isomorphic to $\mathrm{OH}$ ?

Palais' Problem for Operator Spaces (Isometric Version). Let $X$ be a Hilbertian operator space which is a complete extension of $\mathrm{OH}$. Is $\mathrm{X}$ completely isomorphic to $\mathrm{OH}$ ?

\subsection{The operator space structure of the Kalton-Peck spaces}

Recall from Section 4.7 that the $\ell_{p}$ spaces have natural o. s. s.

As a commutative $C^{*}$-algebra, the natural o. s. s. of $\ell_{\infty}$ is the minimal one. The natural $o$. s. s. of $\ell_{1}$, in turn, is given by the inclusion $\ell_{1} \subset\left(\min \left(\ell_{\infty}\right)\right)^{*}$, and agrees with the maximal o. s. s. on $\ell_{1}$.

Following the identity $\left(\ell_{\infty}, \ell_{1}\right)$, the space $\ell_{p}$ has a natural o. s. s. induced by

$$
\ell_{p}=\left(\min \left(\ell_{\infty}\right), \max \left(\ell_{1}\right)\right)_{\theta},
$$

$\theta=\frac{1}{p}$. Let us, as in (BLECHER; LE MERDY, 1995), denote these operator spaces by $O \ell_{p}$. We also have the completely isometric identity $\left(O \ell_{p}\right)^{*}=O \ell_{q}, \frac{1}{p}+\frac{1}{q}=1,1 \leq p<\infty$.

Recall that the Kalton-Peck spaces are the derived spaces of the interpolation process $\left(\ell_{\infty}, \ell_{1}\right)$. Therefore, we have natural o. s. s. on the Kalton-Peck spaces $Z_{p}$, defined by the identification $Z_{p}=d\left(\min \left(\ell_{\infty}\right), \max \left(\ell_{1}\right)\right)_{\theta}, \theta=\frac{1}{p}$. We denote these operator spaces by $O Z_{p}$.

Furthermore, we have extension sequences

$$
0 \longrightarrow O \ell_{p} \longrightarrow O Z_{p} \longrightarrow O \ell_{p} \longrightarrow 0
$$

which are completely singular and completely cosingular, since as twisted sums they are singular and cosingular. 
Notice that by Theorem 5.6.11 we have the completely isomorphic identification $\left(O Z_{p}\right)^{*}=$ $O Z_{q}$.

\subsection{Twisting $\min$ and $\max$}

Suppose we have a short exact sequence of Banach spaces

$$
0 \longrightarrow Y \longrightarrow X \longrightarrow Z \longrightarrow 0
$$

and endow $X$ with any o. s. s., so that 6.1 becomes an extension sequence.

Then we may obtain a complete twisted sum of $\min (Y)$ and $\max (Z)$ in the following way: consider the identity $I d_{Z}: \max (Z) \rightarrow Z$. It is completely bounded, and therefore we may use the pullback construction (Proposition 5.4.2) to obtain a diagram

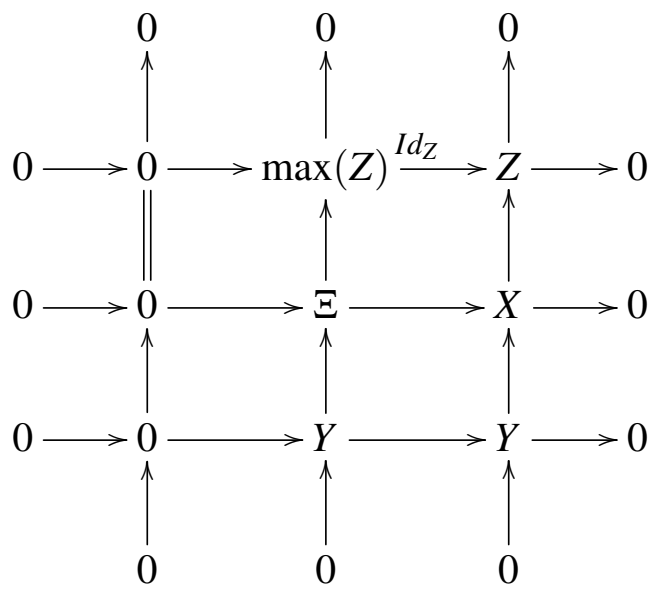

Next, we consider the identity $I d_{Y}: Y \rightarrow \min (Y)$, which is also completely bounded, and therefore we may use the pushout construction (Proposition 5.4.5) to obtain a diagram

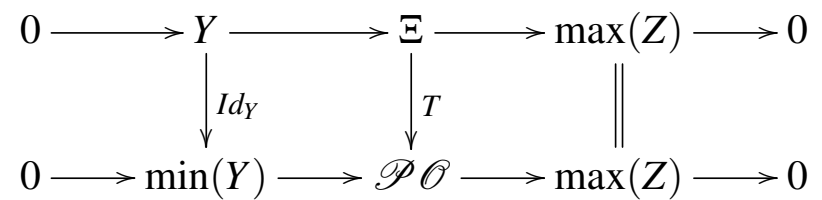

and so we obtain a complete twisted sum of $\min (Y)$ and $\max (Z)$

$$
0 \longrightarrow \min (Y) \longrightarrow \mathscr{P} \mathscr{O} \longrightarrow \max (Z) \longrightarrow 0
$$

So, using the Kalton-Peck spaces (Section 6.1), we may obtain complete twisted sums of $\min \left(\ell_{p}\right)$ and $\max \left(\ell_{p}\right), p \in(0,1)$.

Notice that at the Banach space level we did nothing. Therefore, if the sequence (6.1) is nontrivial, singular or cosingular, so is (6.2).

\subsection{Noncommutative $L_{p}$ spaces}

Let $\mathscr{M}$ be a von Neumann algebra, i. e., a self-adjoint subalgebra with unity of $B(H)$ for some Hilbert space $H$ which is closed in the strong operator topology. We recall that a trace on $\mathscr{M}$ is a map $\tau$ from the set $\mathscr{M}_{+}$of positive elements of $\mathscr{M}$ into $[0, \infty]$ such that for every $x, y \in \mathscr{M}_{+}, \lambda \in[0, \infty)$, we have 
- $\tau(x+y)=\tau(x)+\tau(y)$

- $\tau(\lambda x)=\lambda \tau(x)$

- $\tau\left(x x^{*}\right)=\tau\left(x^{*} x\right)$.

We also suppose that $\tau$ is

- faithful, i. e., $\tau(x)=0$ if and only if $x=0$;

- normal, i. e., for any bounded increasing net $\left(x_{\alpha}\right) \subset \mathscr{M}_{+}$we have $\sup _{\alpha} \tau\left(x_{\alpha}\right)=\tau\left(\sup _{\alpha} x_{\alpha}\right)$;

- semifinite, i. e., any nonzero $x \in \mathscr{M}_{+}$bounds an element of finite trace.

Let $L_{1}(\mathscr{M}, \tau)$ be completion of the space of all $x \in \mathscr{M}$ such that $\tau(|x|)<\infty$ with the norm $\|x\|_{1}=\tau(|x|)$.

The spaces $L_{p}(\mathscr{M}, \tau)$ may be defined by the identity

$$
\left(\mathscr{M}, L_{1}(\mathscr{M}, \tau)\right)_{\theta}=L_{p}(\mathscr{M}, \tau)
$$

for $p=\frac{1}{\theta}$.

The study of the twisted sums induced by the interpolation couple $\left(\mathscr{M}, L_{1}(\mathscr{M}, \tau)\right)$ was carried out in (CABELLO SÁNCHEZ et al., 2016).

In (PISIER, 1998) natural o. s. s. on $\mathscr{M}, L_{1}(\mathscr{M}, \tau)$, and consequently on $L_{p}(\mathscr{M}, \tau)$, were defined. In particular, we have complete twisted sums of the spaces $L_{p}(\mathscr{M}, \tau)$.

\subsection{The spaces $R(\theta)$ and Palais' problem for operator spaces}

In Sections 6.1 and 6.2 we saw examples of extension sequences which are completely singular and completely cosingular. However, these are not satisfactory, since we obtain this information from the Banach space level. This motivates the following nomenclature (we thank Gilles Pisier for the suggestion).

Definition 6.4.1. Let $Y$ and $Z$ be operator spaces. $A$ true twisting of $Y$ and $Z$ is a complete twisted sum $X$ of $Y$ and $Z$ which is trivial but is not completely trivial. If $Y=Z$, we will say that $X$ is a true twisting of $Y$ or a true extension of $Y$.

So, Palais' problem for operator spaces may be rephrased as obtaining a true twisting of $\mathrm{OH}$. In this section we will solve Palais' problem for operator spaces, both the isomorphic and isometric question. Actually, we shall find true extensions of a continuum of non-completely isomorphic Hilbertian operator spaces. But, firstly, we give an example of a true twisting.

Definition 6.4.2. [(PISIER, 1996)] Let $\theta \in[0,1]$. We denote $R(\theta)=(R, C)_{\theta}$.

Lemma 6.4.3. Let $\left(e_{n}\right)$ be an orthonormal system in $\ell_{2}$ and let

$$
x^{n}=\left(\begin{array}{cccc}
e_{1} & e_{2} & \cdots & e_{n} \\
e_{1} & e_{2} & \cdots & e_{n} \\
\vdots & \vdots & \ddots & \vdots \\
e_{1} & e_{2} & \cdots & e_{n}
\end{array}\right) \in M_{n}\left(\ell_{2}\right)
$$

and let $y^{n}=\left(x^{n}\right)^{T}$.

$$
\text { Then }\left\|x^{n}\right\|_{M_{n}(\mathrm{OH})}=\left\|y^{n}\right\|_{M_{n}(\mathrm{OH})}=n^{\frac{3}{4}} .
$$


Proof. For each $k, n$ let $A_{k, n} \in M_{n}$ be the matrix such that each element of column $k$ is 1 and which is 0 elsewhere. By (4.3) we have

$$
\begin{aligned}
\left\|x^{n}\right\|_{M_{n}(O H)}^{2} & =\left\|\sum_{k=1}^{n} A_{k, n} \otimes \overline{A_{k, n}}\right\| \\
& =\left\|\sum_{k=0}^{n-1} A_{k n+k+1, n^{2}}\right\| \\
& =\left\|\sum_{k=1}^{n} A_{k, n^{2}}\right\| \\
& =\left(n^{2}\left\|\sum_{k=1}^{n} A_{k, n}\right\|\right)^{\frac{1}{2}} \\
& =n \sqrt{n}
\end{aligned}
$$

The proof for $y^{n}$ is similar.

Proposition 6.4.4. The operator space $\mathscr{F}(R, C)$ is a true twisting of $\operatorname{ker}\left(\delta_{\frac{1}{2}}\right)$ and $O H=R\left(\frac{1}{2}\right)$. Furthermore, it defines a completely singular extension sequence.

Proof. Let $W$ be a closed infinite dimensional subspace of $O H$ and suppose that $s: W \rightarrow \mathscr{F}$ is a c. b. partial section for $\delta_{\frac{1}{2}}$.

It follows that for every $n \in \mathbb{N}, x \in M_{n}(W)$ and $z \in \overline{\mathbb{S}}$ we have

$$
\left\|s_{n}(x)(z)\right\|_{M_{n}(R(R e(z)))} \leq\left\|s_{n}(x)\right\| \leq\|s\|_{c b}\|x\|_{M_{n}(O H)}
$$

Let $\left(e_{n}\right)$ be an orthonormal basis for $W$ and $P^{n}: \ell_{2} \rightarrow \ell_{2}$ the orthogonal projection onto $\left[e_{1}, \ldots, e_{n}\right]$. Since $P^{n}$ is completely contractive on $R(\theta)$ for every $\theta \in[0,1]$ we have

$$
\left\|\left(P^{n}\right)_{n} s_{n}(x)(z)\right\|_{M_{n}(R(R e(z)))} \leq\|s\|_{c b}\|x\|_{M_{n}(O H)}
$$

Let us show that this would imply $n^{2} \leq\|s\|_{c b}^{2} n \sqrt{n}$ for every $n$. we have

Since $P^{n} \circ P^{m}=P^{n}$ if $n \leq m$ there are functions $a_{i}^{(j)}, i, j \leq 1$, such that for every $n, j \in \mathbb{N}$

$$
P^{n} e_{j}(z)=\sum_{i=1}^{n} a_{i}^{(j)}(z) e_{i}
$$

Notice that each function $a_{i}^{(j)}$ is analytic on $\mathbb{S}$ and bounded on $\overline{\mathbb{S}}$. This implies that for each $n$ the function $g_{n}$ given by

$$
g_{n}(z)=\sum_{j=1}^{n} a_{j}^{(j)}(z) e_{j}
$$

is in $\mathscr{F}$. Notice that $g_{n}\left(\frac{1}{2}\right)=\sum_{i=1}^{n} e_{i}$, so that there is $z_{n} \in \partial \mathbb{S}$ such that

$$
\sum_{j=1}^{n}\left|a_{j}^{(j)}\left(z_{n}\right)\right|^{2} \geq n
$$

Let us suppose that $z_{n} \in \mathbb{S}_{0}$ (if not, use the same argument with $y^{n}$ instead of $x^{n}$ ). 
We have

$$
\left(P^{n}\right)_{n} s_{n} x^{n}\left(z_{n}\right)=\sum_{k=1}^{n}\left(a_{k}^{(j)}\left(z_{n}\right)\right)_{i, j} \otimes e_{k}
$$

Let $A_{n} \in M_{n}$ be the matrix which entries are all 1 . Then

$$
\begin{aligned}
\left\|\left(P^{n}\right)_{n} s_{n} x^{n}\left(z_{n}\right)\right\|_{M_{n}(R)}^{2} & =\left\|\sum_{k=1}^{n}\left(a_{k}^{(j)}\left(z_{n}\right)\right)_{i, j}\left(a_{k}^{(j)}\left(z_{n}\right)\right)_{i, j}^{*}\right\| \\
& \left.=\| \sum_{k=1}^{n}\left(a_{k}^{(j)}\left(z_{n}\right)\right)_{i, j} \overline{\left(a_{k}^{(i)}\left(z_{n}\right)\right.}\right)_{i, j} \| \\
& =\sum_{i, j}\left|a_{i}^{(j)}\left(z_{n}\right)\right|^{2}\left\|A_{n}\right\| \\
& \geq \sum_{j=1}^{n}\left|a_{j}^{(j)}\left(z_{n}\right)\right|^{2}\left\|A_{n}\right\| \\
& \geq n^{2}
\end{aligned}
$$

Together with equation (6.3) and Lemma 6.4.3, this implies $n^{2} \leq\|s\|_{c b}^{2} n \sqrt{n}$ for every $n$, a contradiction.

Therefore, $\delta_{\frac{1}{2}}$ is c. s. s.

We note that the non-complete triviality is also a consequence of Theorem 6.4 .8 below.

\subsubsection{True extensions of $R(\theta)$}

Our goal now is to prove that for each $\theta \in(0,1)$ the interpolation couple $(R, C)$ induces a true twisting of $R(\theta)$.

Lemma 6.4.5. Let $\left(e_{n}\right)$ be an orthonormal system in $\ell_{2}$ and let

$$
x^{n}=\left(\begin{array}{cccc}
e_{1} & e_{2} & \cdots & e_{n} \\
0 & 0 & \cdots & 0 \\
\vdots & \vdots & \ddots & \vdots \\
0 & 0 & \cdots & 0
\end{array}\right) \in M_{n}\left(\ell_{2}\right)
$$

Then $\left\|x^{n}\right\|_{M_{n}(R(\theta))}=n^{\frac{1-\theta}{2}}$ for every $\theta \in[0,1]$.

Proof. Using formulas (4.1) and (4.2) we have that

$$
\left\|x^{n}\right\|_{M_{n}(R)}=n^{\frac{1}{2}}
$$

and

$$
\left\|x^{n}\right\|_{M_{n}(C)}=1
$$

Fix $\theta \in(0,1)$ and let $f_{n} \in M_{n}(\mathscr{F})$ be given by

$$
f_{n}(z)=e^{(z-\theta) \log n^{\frac{1}{2}}} x^{n}
$$

Then $f_{n}(\theta)=x^{n}$ and for every $t \in \mathbb{R}$ we have

$$
\left\|f_{n}(i t)\right\|_{M_{n}(R)}=\left\|f_{n}(1+i t)\right\|_{M_{n}(C)}=n^{\frac{1-\theta}{2}}
$$


so that $\left\|x^{n}\right\|_{M_{n}(R(\theta))} \leq n^{\frac{1-\theta}{2}}$.

Notice that

$$
\left\|\left(\overline{x^{n}}\right)_{n}\left(x^{n}\right)\right\|_{M_{n^{2}}}=n^{\frac{1}{2}}
$$

so that by Lemma 4.2.20 we have

$$
\begin{aligned}
\left\|x^{n}\right\|_{M_{n}(R(\theta))} & =\left\|x^{n}\right\|_{M_{n}(\overline{R(\theta)})} \\
& =\left\|x^{n}\right\|_{M_{n}\left(R(1-\theta)^{*}\right)} \\
& =\left\|x^{n}\right\|_{C B\left(R(1-\theta), M_{n}\right)} \\
& \geq \frac{\left\|\left(\overline{x^{n}}\right)_{n}\left(x^{n}\right)\right\|_{M^{2}}}{\left\|x^{n}\right\|_{M_{n}(R(1-\theta))}} \\
& \geq \frac{n^{\frac{1}{2}}}{n^{\frac{\theta}{2}}} \\
& =n^{\frac{1-\theta}{2}}
\end{aligned}
$$

Definition 6.4.6. For each $\theta \in(0,1)$, let $d R(\theta)=d(R, C)_{\theta}$.

As a consequence of the proof of Lemma 6.4.5 we obtain

Lemma 6.4.7. Let $x^{n}$ be from Lemma 6.4.5. Then $\left\|\left(x^{n}, \log n^{\frac{1}{2}} x^{n}\right)\right\|_{M_{n}(d R(\theta))}=\left\|x^{n}\right\|_{R(\theta)}$ for every $\theta \in(0,1)$.

Proof. Indeed, if we let $f_{n}(z)=e^{(z-\theta) \log n^{\frac{1}{2}}} x^{n}$, then the proof of Lemma 6.4.5 shows that

$$
\left\|f_{n}\right\|=\left\|x^{n}\right\|_{M_{n}(R(\theta))}
$$

This implies that

$$
\left\|x^{n}\right\|_{M_{n}(R(\theta))} \leq\left\|\left(x^{n}, f_{n}^{\prime}(\theta)\right)\right\|_{M_{n}(d R(\theta))} \leq\left\|f_{n}\right\|=\left\|x^{n}\right\|_{M_{n}(R(\theta))}
$$

Since $f_{n}^{\prime}(\theta)=\log n^{\frac{1}{2}} x^{n}$ we obtain

$$
\left\|\left(x^{n}, \log n^{\frac{1}{2}} x^{n}\right)\right\|_{M_{n}(R(\theta))}=\left\|x^{n}\right\|_{R(\theta)}
$$

Theorem 6.4.8. For each $\theta \in(0,1)$, the operator space $d R(\theta)=d(R, C)_{\theta}$ is a true twisting of $R(\theta)$. Furthermore, the extension sequence

$$
0 \longrightarrow R(\theta) \stackrel{i}{\longrightarrow} d R(\theta) \stackrel{q}{\longrightarrow} R(\theta) \longrightarrow 0
$$

is completely singular and completely cosingular.

Proof. Let $W \subset \ell_{2}$ be an infinite dimensional subspace and suppose that $P: q^{-1}(W) \rightarrow q^{-1}(W)$ is a bounded projection with $P\left(q^{-1}(W)\right)=i(R(\theta))$. Then $P$ has the form

$$
P(x, y)=(0, y+T x)
$$

for some $T: W \rightarrow \ell_{2}$ linear. Also

$$
\|T x\|_{\ell_{2}}=\|P(x, 0)\|_{\ell_{2}} \leq\|P\|\|x\|_{\ell_{2}}
$$


so that $T$ is bounded. Since $R(\theta)$ is a homogeneous operator space we have that as a linear map from $W$ into $R(\theta)$

$$
\|T\|_{c b} \leq\|P\|
$$

Fix $n \in \mathbb{N}$ and $y, x \in M_{n}(W)$ and let $\varphi: \mathbb{S} \rightarrow \mathbb{D}$ be a conformal map with $\varphi(\theta)=0$. Recall from the proof of Proposition 5.5.6 that for every $y \in M_{n}(R(\theta))$ we have $\left\|i_{n}(y)\right\|=\frac{\|y\|}{\left|\varphi^{\prime}(\theta)\right|}$. Therefore

$$
\begin{aligned}
\left\|y+T_{n} x\right\| & =\left|\varphi^{\prime}(\theta)\right|\left\|i_{n}\left(y+T_{n} x\right)\right\| \\
& =\left|\varphi^{\prime}(\theta)\right|\left\|\left(0, y+T_{n} x\right)\right\| \\
& =\left|\varphi^{\prime}(\theta)\right|\left\|P_{n}(x, y)\right\| \\
& \leq\left|\varphi^{\prime}(\theta)\right|\left\|P_{n}\right\|\|(x, y)\|
\end{aligned}
$$

If $y=y(x)$ is such that $\|(x, y)\|=\|x\|$, we obtain

$$
\begin{aligned}
\left\|P_{n}\right\|\|x\| & \geq \frac{1}{\left|\varphi^{\prime}(\theta)\right|}\left\|y+T_{n} x\right\| \\
& \geq \frac{1}{\left|\varphi^{\prime}(\theta)\right|}\left(\|y\|-\|T\|_{c b}\|x\|\right)
\end{aligned}
$$

so that

$$
\|y(x)\| \leq\left|\varphi^{\prime}(\theta)\right|\left(\frac{1}{\left|\varphi^{\prime}(\theta)\right|}\|T\|_{c b}+\left\|P_{n}\right\|\right)\|x\|
$$

If we let $x^{n}$ be the matrix from Lemma 6.4.5 for some orthonormal system in $W$, then Lemma 6.4.7 shows that we may choose $y\left(x^{n}\right)=\log n^{\frac{1}{2}} x^{n}$. So, equation (6.5) implies

$$
\log n^{\frac{1}{2}} \leq\|T\|_{c b}+\left|\varphi^{\prime}(\theta)\right|\left\|P_{n}\right\|
$$

so that $P$ cannot be completely bounded. This shows that $d R(\theta)$ is a true twisting of $R(\theta)$, and that it is completely singular.

Complete cosingularity follows from Theorem 5.6.11: we have a complete equivalence of extension sequences

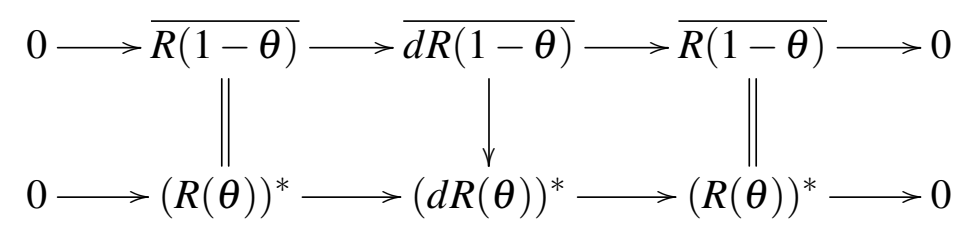

Since the quotient map of the first line is c. s. s., so is the quotient map of the second line, which is the the adjoint of the inclusion map of the extension sequence (6.4). By Proposition 5.3.13, we have that this inclusion map is c. s. c, and therefore (6.4) is completely cosingular. spaces.

Recall that $R\left(\frac{1}{2}\right)=O H$. In this way, we solve the isomorphic Palais' problem for operator

To solve the isometric version, we use the fact that since $R$ and $C$ as Banach spaces are isometric to each other and to $\ell_{2}$, the operator space $\mathscr{F}_{2}^{\frac{1}{2}}(R, C)$ (see definitions 2.5.1 and 5.6.2) is Hilbertian, and therefore so is $d^{2}(R, C)_{\theta}$. By Proposition 5.6.8, we obtain:

Theorem 6.4.9. The space $d^{2}(R, C)_{\frac{1}{2}}$ is a Hilbertian true twisting of $\mathrm{OH}$. 


\subsection{The spaces $o\left(\ell_{p}\right)$}

We show now the existence of true twistings for quantizations of the spaces $\ell_{p}$. The definition of $o(X)$ below is from (PISIER, 1996).

Definition 6.5.1. Let $X$ be a Banach space. We denote $o(X)(\theta)=(\min (X), \max (X))_{\theta}$ and $d o(X)(\theta)=d(\min (X), \max (X))_{\theta}$, for $\theta \in(0,1)$. We also denote $o(X)=o(X)\left(\frac{1}{2}\right)$ and $d o(X)=$ $d o(X)\left(\frac{1}{2}\right)$.

Our objective in this section is to prove that the space $d o\left(\ell_{p}\right)(\theta)$ is a true twisting of $o\left(\ell_{p}\right)(\theta)$ for every $\theta \in(0,1)$ and every $p \in(1, \infty)$.

Lemma 6.5.2. Let $p \in[1, \infty)$, and let $W$ be a complemented infinite dimensional subspace of $\ell_{p}$. If $T: W \rightarrow \ell_{p}$ is an onto isomorphism, then, for every $\theta \in(0,1), T$ is a complete isomorphism between $W$, when viewed as a subspace of $o\left(\ell_{p}\right)(\theta)$, and $o\left(\ell_{p}\right)(\theta)$.

Proof. Let $P: \ell_{p} \rightarrow \ell_{p}$ be a bounded projection with $\operatorname{Im}(P)=W$. Also, let $i: W \rightarrow \ell_{p}$ be the inclusion.

By the homogeneity of the min and max quantizations, we have that $T \circ P: \ell_{p} \rightarrow \ell_{p}$ is completely bounded when viewed as a map from $\min \left(\ell_{p}\right)$ into $\min \left(\ell_{p}\right)$ and when viewed as a map from $\max \left(\ell_{p}\right)$ into $\max \left(\ell_{p}\right)$.

By the interpolation property (Theorem 4.2.15), we have that $T \circ P: o\left(\ell_{p}\right)(\theta) \rightarrow o\left(\ell_{p}\right)(\theta)$ is completely bounded for every $\theta \in(0,1)$.

We also have that $i: W \rightarrow o\left(\ell_{p}\right)(\theta)$ is a complete isometry onto its image when we see $W$ as a subspace of $o\left(\ell_{p}\right)(\theta)$. Therefore, $T=T \circ P \circ i: W \rightarrow o\left(\ell_{p}\right)(\theta)$ is completely bounded.

Furthermore, since $i \circ T^{-1}: \ell_{p} \rightarrow \ell_{p}$ is bounded, it is completely bounded when viewed as a map from $o\left(\ell_{p}\right)(\theta)$ into $o\left(\ell_{p}\right)(\theta)$. So, given $n \in \mathbb{N}$ and $x \in M_{n}\left(\ell_{p}\right)$, we have

$$
\begin{aligned}
\left\|T_{n}^{-1}(x)\right\|_{M_{n}(W)} & =\left\|T_{n}^{-1}(x)\right\|_{M_{n}\left(o\left(\ell_{p}\right)(\theta)\right.} \\
& =\left\|i_{n} \circ T_{n}^{-1}(x)\right\|_{M_{n}\left(o\left(\ell_{p}\right)(\theta)\right.} \\
& \leq\left\|i \circ T^{-1}\right\|_{c b}\|x\|_{M_{n}\left(o \ell_{p}\right)(\theta)}
\end{aligned}
$$

so that $T^{-1}$ is completely bounded, and $T$ is a complete isomorphism.

For the rest of this section we use the following notation.

Notation 6.5.3. Let $\left(e_{n}\right)$ be the canonical basis of $\ell_{2}$. For each $n \in \mathbb{N}$ we denote

$$
x^{n}=\left(\begin{array}{cccc}
e_{1} & e_{2} & \cdots & e_{n} \\
0 & 0 & \cdots & 0 \\
\vdots & \vdots & \ddots & \vdots \\
0 & 0 & \cdots & 0
\end{array}\right) \in M_{n}\left(c_{00}\right)
$$

Lemma 6.5.4. For each $n \in \mathbb{N}$ and $1 \leq p \leq \infty$,

$$
\left\|x^{n}\right\|_{M_{n}\left(\min \left(\ell_{p}\right)\right)}= \begin{cases}n^{\frac{1}{p}-\frac{1}{2}}, & \text { if } 1 \leq p \leq 2 \\ 1, & \text { if } p \geq 2\end{cases}
$$


Proof. From Proposition 4.3.2 we have

$$
\begin{aligned}
\left\|x^{n}\right\|_{M_{n}\left(\min \left(\ell_{p}\right)\right)} & =\sup \left\{\left\|\sum_{i, j} \lambda_{i} \mu_{j} x_{i, j}\right\|_{\ell_{p}}: \sum\left|\lambda_{i}\right|^{2}, \sum\left|\mu_{j}\right|^{2} \leq 1\right\} \\
& =\sup \left\{\left\|\sum_{i=1}^{n} \lambda_{i} e_{i}\right\|_{\ell_{p}}:\left\|\left(\lambda_{i}\right)\right\|_{\ell_{2}}=1\right\} \\
& =\sup \left\{\left\|\left(\lambda_{i}\right)\right\|_{\ell_{p}}:\left\|\left(\lambda_{i}\right)\right\|_{\ell_{2}}=1\right\}
\end{aligned}
$$

and a Lagrange multipliers argument shows that

$$
\left\|x^{n}\right\|_{M_{n}\left(\min \left(\ell_{p}\right)\right)}= \begin{cases}n^{\frac{1}{p}-\frac{1}{2}}, & \text { if } 1 \leq p \leq 2 \\ 1, & \text { if } p \geq 2\end{cases}
$$

Lemma 6.5.5. Let $A_{1}$ be the matrix $(1) \in M_{1}$, and given $A_{n}$, let the matrix $A_{n+1}$ be given by

$$
A_{n+1}=\left(\begin{array}{cc}
1 & \\
\vdots & A_{n} \\
1 & -A_{n}
\end{array}\right)
$$

Then for each $n \in \mathbb{N}$, we have:

1. The scalar product of any two differents columns of $A_{n}$ is 0 .

2. $\left\|A_{n}\right\|=2^{\frac{n-1}{2}}$

Proof. 1 . Write $A_{n}=\left(a_{i, j}^{(n)}\right)_{i, j}$, where $1 \leq i \leq 2^{n-1}$ and $1 \leq j \leq n$. Then we have for $n \geq 1$

$$
a_{i, j}^{(n+1)}= \begin{cases}1, & \text { if } j=1,1 \leq i \leq 2^{n} \\ a_{i, j-1}^{(n)}, & \text { if } 2 \leq j \leq n+1,1 \leq i \leq 2^{n-1} \\ -a_{i-2^{n-1}, j-1}^{(n)}, & \text { if } 2 \leq j \leq n+1,2^{n-1}+1 \leq i \leq 2^{n}\end{cases}
$$

Notice that the scalar product of the two columns of $A_{2}$ is 0 .

Suppose the scalar product of any two different columns of $A_{n}$ is 0 . Let us prove that the same if true for $A_{n+1}$.

Let $1 \leq j<j^{\prime} \leq n+1$. We first suppose $j=1$. Then

$$
\begin{aligned}
\sum_{i=1}^{2^{n}} a_{i, 1}^{(n+1)} a_{i, j^{\prime}}^{(n+1)} & =\sum_{i=1}^{2^{n}} a_{i, j^{\prime}}^{(n+1)} \\
& =\sum_{i=1}^{2^{n-1}} a_{i, j^{\prime}-1}^{(n)}-\sum_{i=2^{n}-1+1}^{2^{n}} a_{i-2^{n-1}, j^{\prime}-1}^{(n)} \\
& =\sum_{i=1}^{2^{n-1}}\left(a_{i, j^{\prime}-1}^{(n)}-a_{i, j^{\prime}-1}^{(n)}\right) \\
& =0
\end{aligned}
$$

Suppose now that $j>1$. Then, by induction hypothesis

$$
\sum_{i=1}^{2^{n}} a_{i, j}^{(n+1)} a_{i, j^{\prime}}^{(n+1)}=\sum_{i=1}^{2^{n-1}} a_{i, j-1}^{(n)} a_{i, j^{\prime}-1}^{(n)}+\sum_{i=2^{n-1}+1}^{2^{n}} a_{i-2^{n-1}, j-1}^{(n)} a_{i-2^{n-1}, j^{\prime}-1}^{(n+1)}
$$




$$
\begin{aligned}
& =2 \sum_{i=1}^{2^{n-1}} a_{i, j-1}^{(n)} a_{i, j^{\prime}-1}^{(n)} \\
& =0
\end{aligned}
$$

2. By 1 . we have that $A_{n}^{*} A_{n}=2^{n-1} I d_{n}$, where $I d_{n} \in M_{n}$ is the identity matrix. Therefore

$$
\left\|A_{n}\right\|=\left\|A_{n}^{*} A_{n}\right\|^{\frac{1}{2}}=2^{\frac{n-1}{2}}
$$

Lemma 6.5.6. For each $n \in \mathbb{N}$ and $1 \leq p \leq \infty$,

$$
\left\|x^{n}\right\|_{M_{n}\left(\max \left(\ell_{p}\right)\right)}= \begin{cases}n^{\frac{1}{2}}, & \text { if } 1 \leq p \leq 2 \\ n^{\frac{1}{p}}, & \text { if } p \geq 2\end{cases}
$$

Proof. Let $A \in M_{n, 2^{n-1}}$ be given by

$$
A=\frac{1}{2^{n-1}}\left(\begin{array}{cccc}
1 & 1 & \cdots & 1 \\
0 & 0 & \cdots & 0 \\
\vdots & \vdots & \ddots & \vdots \\
0 & 0 & \cdots & 0
\end{array}\right)
$$

Let $A_{n}$ be the matrix from Lemma 6.5.5, and write $A_{n}=\left(a_{i, j}^{(n)}\right)$. Let $D \in M_{2^{n-1}}\left(\ell_{p}\right)=\left(d_{i, j}\right)$ be given by

$$
d_{i, j}= \begin{cases}\sum_{l=1}^{n} a_{i, l}^{(n)}, & \text { if } i=j \\ 0, & \text { otherwise }\end{cases}
$$

We claim that $x^{n}=A D A_{n}$. Indeed, we have

$$
A D=\frac{1}{2^{n-1}}\left(\begin{array}{cccc}
d_{1,1} & d_{2,2} & \cdots & d_{2^{n-1}, 2^{n-1}} \\
0 & 0 & \cdots & 0 \\
\vdots & \vdots & \ddots & \vdots \\
0 & 0 & \cdots & 0
\end{array}\right) \in M_{n, 2^{n-1}}\left(\ell_{p}\right)
$$

Then, we have $\left(A D A_{n}\right)_{i, j}=0$ if $i>1$, and by 1 . of Lemma 6.5.5

$$
\begin{aligned}
2^{n-1}\left(A D A_{n}\right)_{1, j} & =\sum_{k=1}^{2^{n-1}} d_{k, k} a_{k, j}^{(n)} \\
& =\sum_{k=1}^{2^{n-1}} \sum_{l=1}^{n} a_{k, l}^{(n)} a_{k, j}^{(n)} e_{l} \\
& =\sum_{l=1}^{n} \sum_{k=1}^{2^{n-1}} a_{k, l}^{(n)} a_{k, j}^{(n)} e_{l} \\
& =\sum_{k=1}^{2^{n-1}} a_{k, j}^{(n)} a_{k, j}^{(n)} e_{j} \\
& =2^{n-1} e_{j}
\end{aligned}
$$


Therefore, $A D A_{n}=x^{n}$. Proposition 4.3.3 and Lemma 6.5.5 imply that

$$
\begin{aligned}
\left\|x^{n}\right\|_{M_{n}\left(\max \left(\ell_{p}\right)\right)} & \leq\|A\|\|D\|\left\|A_{n}\right\| \\
& =\frac{1}{2^{n-1}} 2^{\frac{n-1}{2}} n^{\frac{1}{p}} 2^{\frac{n-1}{2}} \\
& =n^{\frac{1}{p}}
\end{aligned}
$$

Recall, however, that $\left\|x^{n}\right\|_{M_{n}(R)}=n^{\frac{1}{2}}$. Therefore, $\left\|x^{n}\right\|_{M_{n}\left(\max \left(\ell_{2}\right)\right)}=n^{\frac{1}{2}}$.

Let $1 \leq p<2$. By Proposition 4.3.7 the contraction $i: \ell_{p} \rightarrow \ell_{2}$ is a complete contraction $i: \max \left(\ell_{p}\right) \rightarrow \max \left(\ell_{2}\right)$, so that

$$
\left\|x^{n}\right\|_{M_{n}\left(\max \left(\ell_{p}\right)\right)} \geq n^{\frac{1}{2}}
$$

Notice also that we have a decomposition $x^{n}=B C I d_{n}$, where

$$
B=\left(\begin{array}{cccc}
1 & 1 & \cdots & 1 \\
0 & 0 & \cdots & 0 \\
\vdots & \vdots & \ddots & \vdots \\
0 & 0 & \cdots & 0
\end{array}\right) \in M_{n}
$$

and

$$
C=\left(\begin{array}{cccc}
e_{1} & 0 & \cdots & 0 \\
0 & e_{2} & \cdots & 0 \\
\vdots & \vdots & \ddots & \vdots \\
0 & 0 & \cdots & e_{n}
\end{array}\right) \in M_{n}\left(\ell_{p}\right)
$$

so that $\left\|x^{n}\right\|_{M_{n}\left(\max \left(\ell_{p}\right)\right)}$ is at most $n^{\frac{1}{2}}$, again by Proposition 4.3.3. Therefore, for $1 \leq p<2$ we have $\left\|x^{n}\right\|_{M_{n}\left(\max \left(\ell_{p}\right)\right)}=n^{\frac{1}{2}}$.

For the case $p>2$, recall that $M_{n}\left(\max \left(\ell_{p}\right)\right)=M_{n}\left(\left(\min \left(\ell_{q}\right)\right)^{*}\right)=C B\left(\min \left(\ell_{q}\right), M_{n}\right), \frac{1}{p}+$ $\frac{1}{q}=1$. Then, by Lemma 6.5.4

$$
\begin{aligned}
\left\|x^{n}\right\|_{M_{n}\left(\max \left(\ell_{p}\right)\right)} & \geq \frac{\left\|\left(x^{n}\right)_{n}\left(x^{n}\right)\right\|}{\left\|x^{n}\right\|_{M_{n}\left(\min \left(\ell_{q}\right)\right)}} \\
& =\frac{n^{\frac{1}{2}}}{n^{\frac{1}{q}-\frac{1}{2}}} \\
& =n^{\frac{1}{p}}
\end{aligned}
$$

and the result is proved.

Lemma 6.5.7. For each $n \in \mathbb{N}, 0<\theta<1$ and $1 \leq p \leq \infty$,

$$
\left\|x^{n}\right\|_{M_{n}\left(o\left(\ell_{p}\right)(\theta)\right)}= \begin{cases}n^{\frac{1}{2}-(1-\theta)\left(1-\frac{1}{p}\right)}, & \text { if } 1 \leq p \leq 2 \\ n^{\frac{\theta}{p}}, & \text { if } p \geq 2\end{cases}
$$

Proof. Fix $p$ and $\theta$ and let

$$
\lambda_{n}= \begin{cases}n^{1-\frac{1}{p}}, & \text { if } 1 \leq p \leq 2 \\ n^{\frac{1}{p}}, & \text { if } p \geq 2\end{cases}
$$


Let $f_{n}(z)=e^{(z-\theta) \log \left(\lambda_{n}^{-1}\right)} x^{n}$. Then $f \in M_{n}\left(\mathscr{F}\left(\min \left(\ell_{p}\right), \max \left(\ell_{p}\right)\right)\right)$. By Lemma 6.5.4 we have for every $t \in \mathbb{R}$

$$
\begin{aligned}
\|f(i t)\|_{M_{n}\left(\min \left(\ell_{p}\right)\right)} & =e^{-\theta \log \left(\lambda_{n}^{-1}\right)}\left\|x^{n}\right\|_{M_{n}\left(\min \left(\ell_{p}\right)\right)} \\
& = \begin{cases}n^{\theta\left(1-\frac{1}{p}\right)} n^{\frac{1}{p}-\frac{1}{2}}, & \text { if } 1 \leq p \leq 2 \\
n^{\frac{\theta}{p}}, & \text { if } p \geq 2\end{cases} \\
& = \begin{cases}n^{\frac{1}{2}-(1-\theta)\left(1-\frac{1}{p}\right)}, & \text { if } 1 \leq p \leq 2 \\
n^{\frac{\theta}{p}}, & \text { if } p \geq 2\end{cases}
\end{aligned}
$$

and by Lemma 6.5.6 we have for every $t \in \mathbb{R}$

$$
\begin{aligned}
\|f(1+i t)\|_{M_{n}\left(\max \left(\ell_{p}\right)\right)} & =e^{(1-\theta) \log \left(\lambda_{n}^{-1}\right)}\left\|x^{n}\right\|_{M_{n}\left(\min \left(\ell_{p}\right)\right)} \\
& = \begin{cases}n^{-(1-\theta)\left(1-\frac{1}{p}\right)} n^{\frac{1}{2}}, & \text { if } 1 \leq p \leq 2 \\
n^{-\frac{1-\theta}{p}} n^{\frac{1}{p}}, & \text { if } p \geq 2\end{cases} \\
& = \begin{cases}n^{\frac{1}{2}-(1-\theta)\left(1-\frac{1}{p}\right)}, & \text { if } 1 \leq p \leq 2 \\
n^{\frac{\theta}{p}}, & \text { if } p \geq 2\end{cases}
\end{aligned}
$$

Also, $f_{n}(\theta)=x^{n}$. This implies that

$$
\left\|x^{n}\right\|_{M_{n}\left(o\left(\ell_{p}\right)(\theta)\right)} \leq \begin{cases}n^{\frac{1}{2}-(1-\theta)\left(1-\frac{1}{p}\right)}, & \text { if } 1 \leq p \leq 2 \\ n^{\frac{\theta}{p}}, & \text { if } p \geq 2\end{cases}
$$

For the other inequality, from Theorem 4.2.19 we have completely isometrically

$$
\begin{aligned}
o\left(\ell_{p}\right)(\theta) & =\left(\min \left(\ell_{p}\right), \max \left(\ell_{p}\right)\right)_{\theta} \\
& =\left(\max \left(\ell_{q}\right)^{*}, \min \left(\ell_{q}\right)^{*}\right)_{\theta} \\
& =\left(\left(\max \left(\ell_{q}\right), \min \left(\ell_{q}\right)\right)_{\theta}\right)^{*} \\
& =\left(\left(\min \left(\ell_{q}\right), \max \left(\ell_{q}\right)_{1-\theta}\right)^{*}\right. \\
& =o\left(\ell_{q}\right)(1-\theta)^{*}
\end{aligned}
$$

where $\frac{1}{p}+\frac{1}{q}=1$ (for $p=1$, we have $o\left(\ell_{p}\right)(\theta)=o\left(c_{0}\right)(1-\theta)^{*}$ ), so that

$$
\begin{aligned}
\left\|x^{n}\right\|_{M_{n}\left(o\left(\ell_{p}\right)(\theta)\right)} & =\left\|x^{n}\right\|_{M_{n}\left(o\left(\ell_{q}\right)(1-\theta)^{*}\right)} \\
& =\left\|x^{n}\right\|_{C B\left(o\left(\ell_{q}\right)(1-\theta)^{*}, M_{n}\right)} \\
& \geq \frac{\left\|\left(x^{n}\right)_{n}\left(x^{n}\right)\right\|}{\left\|x^{n}\right\|_{o\left(\ell_{q}\right)(1-\theta)}} \\
& \geq \begin{cases}\frac{n^{\frac{1}{2}}}{n^{1-\theta} q}, & \text { if } 1 \leq p \leq 2 \\
\frac{n^{\frac{1}{2}}}{n^{\frac{1}{2}-\theta\left(1-\frac{1}{q}\right)},} & \text { if } p \geq 2\end{cases} \\
& = \begin{cases}n^{\frac{1}{2}-(1-\theta)\left(1-\frac{1}{p}\right)}, & \text { if } 1 \leq p \leq 2 \\
n^{\frac{\theta}{p}}, & \text { if } p \geq 2\end{cases}
\end{aligned}
$$

and the result is proved. 
As a corollary of the proof of Lemma 6.5.7 we obtain

Corollary 6.5.8. Let $1 \leq p \leq \infty, \theta \in(0,1)$ and $n \in \mathbb{N}$. Then

$$
\left\|x^{n}\right\|_{M_{n}\left(o\left(\ell_{p}\right)(\theta)\right)}= \begin{cases}\left\|\left(x^{n}, \log \left(n^{-\left(1-\frac{1}{p}\right)}\right) x^{n}\right)\right\|_{M_{n}\left(d o\left(\ell_{p}\right)(\theta)\right)}, & \text { if } 1 \leq p \leq 2 \\ \left.\|\left(x^{n}, \log \left(n^{-\frac{1}{p}}\right)\right) x^{n}\right) \|_{M_{n}\left(\operatorname{do}\left(\ell_{p}\right)(\theta)\right)}, & \text { if } p \geq 2\end{cases}
$$

Proof. Fix $p$ and $\theta$ and let

$$
\lambda_{n}= \begin{cases}n^{1-\frac{1}{p}}, & \text { if } 1 \leq p \leq 2 \\ n^{\frac{1}{p}}, & \text { if } p \geq 2\end{cases}
$$

Let $f_{n}(z)=e^{(z-\theta) \log \left(\lambda_{n}^{-1}\right)} x^{n}$. Then $f \in M_{n}\left(\mathscr{F}\left(\min \left(\ell_{p}\right), \max \left(\ell_{p}\right)\right)\right), f_{n}(\theta)=x^{n}$, and by the proof of Lemma 6.5.7 we have that $\left\|f_{n}\right\|=\left\|x^{n}\right\|_{M_{n}\left(o\left(\ell_{p}\right)(\theta)\right)}$. Therefore, $\left\|x^{n}\right\|_{M_{n}\left(o\left(\ell_{p}\right)(\theta)\right)}=$ $\left\|\left(f(\theta), f^{\prime}(\theta)\right)\right\|_{M_{n}\left(\operatorname{do}\left(\ell_{p}\right)(\theta)\right)}$.

Theorem 6.5.9. For every $p \in(1, \infty)$ and every $\theta \in(0,1)$ the extension sequence

$$
0 \longrightarrow o\left(\ell_{p}\right)(\theta) \stackrel{i}{\longrightarrow} d o\left(\ell_{p}\right)(\theta) \stackrel{q}{\longrightarrow} o\left(\ell_{p}\right)(\theta) \longrightarrow 0
$$

induced by complex interpolation is completely cosingular and completely singular.

Proof. Let $W$ be a closed infinite dimensional subspace of $o \ell_{p}(\theta)$ and consider the extension sequence

$$
0 \longrightarrow o\left(\ell_{p}\right)(\theta) \stackrel{i}{\longrightarrow} q^{-1}(W) \stackrel{p}{\longrightarrow} W \longrightarrow 0
$$

where $p$ is the restriction of $q$ to $q^{-1}(W)$, and suppose that this extension sequence is completely trivial.

Since $\ell_{p}$ is $\ell_{p}$-saturated (i. e., every closed infinite dimensional subspace of $\ell_{p}$ has a further subspace isomorphic to $\ell_{p}$ ) we may suppose that $W$ is isomorphic to $\ell_{p}$, and the complete triviality is preserved.

Let $T: W \rightarrow \ell_{p}$ be an isomorphism. Then by Lemma 6.5.2 $T: W \rightarrow o\left(\ell_{p}\right)(\theta)$ is a complete isomorphism.

Let $P: q^{-1}(W) \rightarrow q^{-1}(W)$ be a bounded projection with $P\left(q^{-1}(W)\right)=i\left(o\left(\ell_{p}\right)(\theta)\right)$. Then $P$ has the form

$$
P(x, y)=\left(0, y+T^{\prime} x\right)
$$

for some $T^{\prime}: W \rightarrow \ell_{p}$ linear. Also

$$
\left\|T^{\prime} x\right\|_{\ell_{p}}=\|P(x, 0)\|_{\ell_{p}} \leq\|P\|\|x\|_{\ell_{p}}
$$

so that $T^{\prime}$ is bounded. By homogeneity (Proposition 4.3.7) we have that $T^{\prime}$ is completely bounded from $W \subset \min \left(\ell_{p}\right)$ into $\min \left(\ell_{p}\right)$ and from $W \subset \max \left(\ell_{p}\right)$ into $\max \left(\ell_{p}\right)$. By interpolation (Theorem 4.2.15) $T^{\prime}$ is completely bounded from $W \subset o\left(\ell_{p}\right)(\theta)$ into $o\left(\ell_{p}\right)(\theta)$ (recall that $W$ is complemented in $\ell_{p}$ ). As a side note, notice that if $W=\ell_{p}$ then

$$
\left\|T^{\prime}\right\|_{c b} \leq\|P\|
$$

Fix $n \in \mathbb{N}, x \in M_{n}(W)$ and $y \in M_{n}\left(o\left(\ell_{p}\right)(\theta)\right)$, and let $\varphi: \mathbb{S} \rightarrow \mathbb{D}$ be a conformal map with $\varphi(\theta)=$ 0 . From the proof of Proposition 5.5.6 we have for every $y \in M_{n}\left(o\left(\ell_{p}\right)(\theta)\right)$ that $\left\|i_{n}(y)\right\|=\frac{\|y\|}{\left|\varphi^{\prime}(\theta)\right|}$. Therefore

$$
\left\|y+T_{n}^{\prime} x\right\|=\left|\varphi^{\prime}(\theta)\right|\left\|i_{n}\left(y+T_{n}^{\prime} x\right)\right\|
$$




$$
\begin{aligned}
& =\left|\varphi^{\prime}(\theta)\right|\left\|\left(0, y+T_{n}^{\prime} x\right)\right\| \\
& =\left|\varphi^{\prime}(\theta)\right|\left\|P_{n}(x, y)\right\| \\
& \leq\left|\varphi^{\prime}(\theta)\right|\left\|P_{n}\right\|\|(x, y)\|
\end{aligned}
$$

so that

$$
\|y\| \leq\left|\varphi^{\prime}(\theta)\right|\left\|P_{n}\right\|\|(x, y)\|+\left\|T^{\prime}\right\|_{c b}\|x\|
$$

Let $f \in M_{n}(\mathscr{F})$ be such that $f(\theta)=x$ and $f^{\prime}(\theta)=y$. Consider the function $g \in M_{n}(\mathscr{F})$ given by $g(z)=T_{n}^{-1} f(z)$. Then $g(\theta)=T_{n}^{-1} x, g^{\prime}(\theta)=T_{n}^{-1} y$ and $\|g\| \leq\left\|T^{-1}\right\|_{c b}\|f\|$. This implies that

$$
\left\|\left(T_{n}^{-1} x, T_{n}^{-1} y\right)\right\| \leq\left\|T^{-1}\right\|_{c b}\|(x, y)\|
$$

Given $n$, take $x=T_{n}^{-1} x^{n}$ and $y=\log \mu_{n} T_{n}^{-1} x^{n}$, where $\mu_{n}$ is from Corollary 6.5.8 such that $\left\|\left(x^{n}, \log \mu_{n} x^{n}\right)\right\|=\left\|x^{n}\right\|$.

Then equations (6.7) and (6.8) imply

$$
\begin{aligned}
\left|\log \mu_{n}\right|\left\|x^{n}\right\| & \leq\|T\|_{c b}\|y\| \\
& \leq\left(\left|\varphi^{\prime}(\theta)\right|\left\|P_{n}\right\|\|(x, y)\|+\left\|T^{\prime}\right\|_{c b}\|x\|\right)\|T\|_{c b} \\
& \leq\left(\left|\varphi^{\prime}(\theta)\right|\left\|P_{n}\right\|\left\|T^{-1}\right\|_{c b}\left\|\left(x^{n}, \log \mu_{n} x^{n}\right)\right\|+\left\|T^{\prime}\right\|_{c b}\left\|T^{-1}\right\|_{c b}\left\|x^{n}\right\|\right)\|T\|_{c b} \\
& =\left\|T^{-1}\right\|_{c b}\left(\left|\varphi^{\prime}(\theta)\right|\left\|P_{n}\right\|+\left\|T^{\prime}\right\|_{c b}\right)\left\|x^{n}\right\|\|T\|_{c b}
\end{aligned}
$$

so that

$$
\left|\log \mu_{n}\right| \leq\left\|T^{-1}\right\|_{c b}\left(\left|\varphi^{\prime}(\theta)\right|\left\|P_{n}\right\|+\left\|T^{\prime}\right\|_{c b}\right)\|T\|_{c b}
$$

which implies that $P$ is not completely bounded. Therefore, the extension sequence (6.6) is completely singular.

As in Theorem 6.4.8, complete cosingularity is proved by duality. By Theorem 5.6.11 we have a complete equivalence of extension sequences

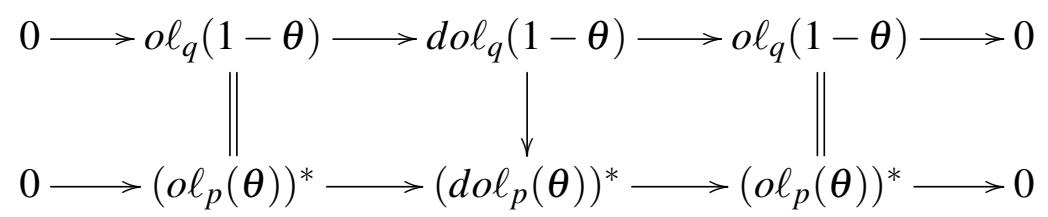

and since the quotient map of the first line is c. s. s., by Proposition 5.3.12 so is the quotient of the second, which by Proposition 5.3.13 implies that the inclusion in (6.6) is c. s. c.

\subsection{Complete extensions of $\mathrm{OH}$}

By Theorem 4.2.17, we have the completely isometric identity $o\left(\ell_{2}\right)=O H$. So, we have a complete extension of $\mathrm{OH}$

$$
0 \longrightarrow \mathrm{OH} \longrightarrow \mathrm{do}\left(\ell_{2}\right) \longrightarrow \mathrm{OH} \longrightarrow 0
$$

Notation 6.6.1. $d O H=d R\left(\frac{1}{2}\right)$

We will show now that $d \mathrm{OH}$ and $d o\left(\ell_{2}\right)$ are not completely isomorphic equivalent.

For the rest of this section we will use the following notation: 
Notation 6.6.2. Let $\left(e_{n}\right)$ be the canonical basis of $\ell_{2}$ and for each $n \in \mathbb{N}$ let $y^{n} \in M_{n}\left(\ell_{2}\right)$ be given by

$$
y^{n}=\left(\begin{array}{cccc}
e_{1} & e_{2} & \cdots & e_{n} \\
\vdots & \vdots & \ddots & \vdots \\
e_{n^{2}-n+1} & e_{n^{2}-n+2} & \cdots & e_{n^{2}}
\end{array}\right)
$$

Lemma 6.6.3 ((PAULSEN, 2002), commentary after Proposition 8.11). Let $X$ be an operator space. If $\varphi: X \rightarrow M_{n}$, then $\|\varphi\|_{c b} \leq n\|\varphi\|$.

Lemma 6.6.4. For every $n \in \mathbb{N}$ we have

1. $\left\|y^{n}\right\|_{M_{n}\left(\min \left(\ell_{2}\right)\right)}=1$;

2. $\left\|y^{n}\right\|_{M_{n}(R)}=\left\|y^{n}\right\|_{M_{n}(C)}=\left\|y^{n}\right\|_{M_{n}(\mathrm{OH})}=n^{\frac{1}{2}}$;

3. $\left\|y^{n}\right\|_{M_{n}\left(\max \left(\ell_{2}\right)\right)}=n$.

Proof. 1. From Proposition 4.3.2 we have

$$
\begin{aligned}
\left\|y^{n}\right\|_{M_{n}\left(\min \left(\ell_{2}\right)\right)} & =\sup \left\{\left\|\sum_{i, j} \lambda_{i} \mu_{j} y_{i, j}\right\|_{\ell_{2}}:\|\lambda\|_{\ell_{2}},\|\mu\|_{\ell_{2}} \leq 1\right\} \\
& =\sup \left\{\left(\sum_{i, j}\left|\lambda_{i} \mu_{j}\right|^{2}\right)^{\frac{1}{2}}:\|\lambda\|_{\ell_{2}},\|\mu\|_{\ell_{2}} \leq 1\right\} \\
& \leq \sup \left\{\|\lambda\|_{\ell_{2}}:\|\lambda\|_{\ell_{2}} \leq 1\right\} \\
& =1
\end{aligned}
$$

and by taking $\lambda_{1}=\mu_{1}=1, \lambda_{i}=\mu_{i}=0$ for $i>1$, we have that the sup is 1 .

2. Let $E_{i, j} \in M_{n}$ be the matrix which $i, j$-entry is 1 and the other entries are 0 . Then, by

$$
\begin{aligned}
\left\|y^{n}\right\|_{M_{n}(R)}^{2} & =\left\|\sum_{1 \leq i, j \leq n} E_{i, j} E_{i, j}^{*}\right\| \\
& =\left\|\sum_{1 \leq i, j \leq n} E_{i, j} E_{j, i}\right\| \\
& =\left\|\sum_{1 \leq i, j \leq n} E_{i, i}\right\| \\
& =\left\|n I d_{n}\right\| \\
& =n
\end{aligned}
$$

A similar calculation shows that $\left\|y^{n}\right\|_{M_{n}(C)}=n^{\frac{1}{2}}$. Let $F_{i, j} \in M_{n^{2}}$ be such that its $i, j$-entry is 1 and the other entries are 0 . We have

$$
\begin{aligned}
\left\|y^{n}\right\|_{M_{n}(O H)}^{2} & =\left\|\sum_{1 \leq i, j \leq n} E_{i, j} \otimes \overline{E_{i, j}}\right\| \\
& =\left\|\sum_{1 \leq i, j \leq n} F_{(i-1) n+i,(j-1) n+j}\right\| \\
& =\left\|\sum_{1 \leq i, j \leq n} E_{i, j}\right\| \\
& =n
\end{aligned}
$$

3. We have isometrically $M_{n}\left(\max \left(\ell_{2}\right)\right)=M_{n}\left(\left(\min \left(\ell_{2}\right)^{*}\right)=C B\left(\min \left(\ell_{2}\right), M_{n}\right)\right.$, so that by Lemma 6.6.3

$$
\left\|y^{n}\right\|_{M_{n}\left(\max \left(\ell_{2}\right)\right)} \leq n\left\|y^{n}\right\|_{\ell_{2} \rightarrow M_{n}}
$$


But, by Proposition 4.3.1 and 1.,

$$
\begin{aligned}
\left\|y^{n}\right\|_{\ell_{2} \rightarrow M_{n}} & =\sup \left\{\left\|y^{n}(x)\right\|: x \in \ell_{2},\|x\|=1\right\} \\
& =\sup \left\{\left\|(x)_{n}\left(y^{n}\right)\right\|: x \in \ell_{2},\|x\|=1\right\} \\
& =\left\|y^{n}\right\|_{M_{n}\left(\min \left(\ell_{2}\right)\right)} \\
& =1
\end{aligned}
$$

so that $\left\|y^{n}\right\|_{M_{n}\left(\max \left(\ell_{2}\right)\right)} \leq n$. But, again by 1 .,

$$
\begin{aligned}
\left\|y^{n}\right\|_{M_{n}\left(\ell_{2}\right)} & \geq\left\|\left(y^{n}\right)_{n} y^{n}\right\| \\
& =\left\|\sum_{1 \leq i, j \leq n} E_{i, j}\right\| \\
& =n
\end{aligned}
$$

and the result is proved.

Lemma 6.6.5. For every $n \in \mathbb{N}$ we have

$$
\left\|y^{n}\right\|_{M_{n}(\mathrm{OH})}=\left\|\left(y^{n}, \log \left(n^{-1}\right) y^{n}\right)\right\|_{M_{n}\left(d o\left(\ell_{2}\right)\right)}
$$

Proof. Let $F_{n}(z)=e^{\log \left(n^{-1}\right)\left(z-\frac{1}{2}\right)} y^{n}$. Then $F_{n}\left(\frac{1}{2}\right)=y^{n}$, and by Lemma 6.6.4 we have $\left\|F_{n}\right\|=$ $\left\|y^{n}\right\|_{M_{n}(O H)}$. Therefore $\left\|y^{n}\right\|_{M_{n}(O H)}=\left\|\left(y^{n}, F_{n}^{\prime}\left(\frac{1}{2}\right)\right)\right\|_{M_{n}\left(d o\left(\ell_{2}\right)\right)}$, and the result is proved.

Theorem 6.6.6. The complete extensions $d R\left(\frac{1}{2}\right)$ and $d o\left(\ell_{2}\right)$ of $\mathrm{OH}$ are not completely isomorphically equivalent.

Proof. Suppose we have a commutative diagram

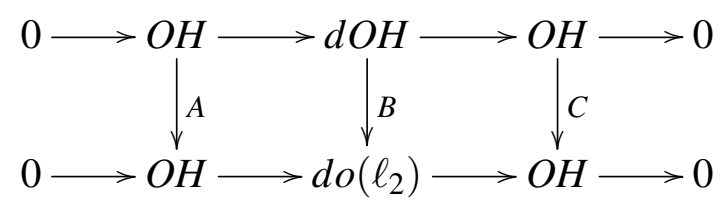

where the vertical arrows are complete isomorphisms. Then $B$ must have the form

$$
B(x, y)=(C(x), A(y)+T(x))
$$

for some $T: O H \rightarrow O H$. Since the extension sequences are trivial as twisted sums of Banach spaces and the projection from $d o\left(\ell_{2}\right)$ onto $O H$ may be taken as $(x, y) \mapsto y$ there is some constant $K>0$ such that

$$
\|T(x)\| \leq K\|B(x, 0)\| \leq K\|B\|\|(x, 0)\|=K\|B\|\|x\|
$$

so that $T$ is bounded, and by the homogeneity of $\mathrm{OH}$ (Proposition 4.4.3) it is completely bounded.

By Lemma 6.6.4, we have that

$$
\begin{aligned}
\left\|\left(C_{n} y^{n}, T_{n} y^{n}\right)\right\|_{M_{n}\left(\operatorname{do}\left(\ell_{2}\right)\right)} & =\left\|B_{n}\left(y^{n}, 0\right)\right\|_{M_{n}\left(d o\left(\ell_{2}\right)\right)} \\
& \leq\|B\|_{c b}\left\|\left(y^{n}, 0\right)\right\|_{M_{n}(d O H)} \\
& =\|B\|_{c b}\left\|y^{n}\right\|_{M_{n}(O H)}
\end{aligned}
$$

Let $\varphi: \mathbb{S} \rightarrow \mathbb{D}$ be a conformal map such that $\varphi\left(\frac{1}{2}\right)=0$ and let $\beta=\frac{1}{\varphi^{\prime}\left(\frac{1}{2}\right)}$. Then we have by Lemma 6.6.5

$\left\|\left(C_{n} y^{n}, T_{n} y^{n}\right)\right\|_{M_{n}\left(d o\left(\ell_{2}\right)\right)} \geq \frac{1}{\left\|B^{-1}\right\|_{c b}}\left\|\left(y^{n}, 0\right)\right\|_{M_{n}(d O H)}$ 


$$
\begin{aligned}
& \geq \frac{1}{\left\|B^{-1}\right\|_{c b}}\left(\|\left(0,-\log \left(n^{-1}\right) y^{n}\left\|_{M_{n}(d O H)}-\right\|\left(y^{n}, \log \left(n^{-1}\right) y^{n} \|_{M_{n}(d O H)}\right)\right.\right. \\
& =\frac{1}{\left\|B^{-1}\right\|_{c b}}(\beta \log n-1)\left\|y^{n}\right\|_{M_{n}(d O H)}
\end{aligned}
$$

so that we obtain

$$
\|B\|_{c b}\left\|B^{-1}\right\|_{c b} \geq \beta \log n-1
$$

for every $n$, a contradiction.

Corollary 6.6.7. Let $\eta \in[0,1], \eta \neq \frac{1}{2}$. Then there is no $\theta \in[0,1]$ such that $o\left(\ell_{2}\right)(\theta)=R(\eta)$, where equality is in the sense of existing an invertible self-adjoint operator $T: \ell_{2} \rightarrow \ell_{2}$ such that $T$ induces a complete isometry between $o\left(\ell_{2}\right)(\theta)$ and $R(\eta)$.

Proof. Suppose that there is $\theta \in[0,1]$ such that $o\left(\ell_{2}\right)(\theta)=R(\eta)$. Since $\eta \neq \frac{1}{2}$, we have that $\theta \neq \frac{1}{2}$ as well. By duality, we have

$$
R(1-\eta)=\overline{R(\eta)^{*}}=\overline{o\left(\ell_{2}\right)(\theta)^{*}}=o\left(\ell_{2}\right)(1-\eta)
$$

Let

- $X_{0}=o\left(\ell_{2}\right)(\theta)$

- $X_{1}=o\left(\ell_{2}\right)(1-\theta)$

- $Y_{0}=R(\eta)$

- $Y_{1}=R(1-\eta)$

By an adaptation of the Reiteration Theorem 5.5.8 we would have that the extension sequence defining $d O H$ would be completely projectively equivalent to the one of $d o\left(\ell_{2}\right)$, contradicting Theorem 6.6.6.

Proposition 6.6.8. The operator spaces $d O H$ and $d o\left(\ell_{2}\right)$ are each completely isomorphic to their conjugate duals.

Proof. We prove for $d O H$, the proof for $d o\left(\ell_{2}\right)$ being similar. By Theorem 5.6.11 we have completely isomorphically

$$
\begin{aligned}
\overline{d O H^{*}} & =\overline{\left(d(R, C)_{\frac{1}{2}}\right)^{*}} \\
& =\overline{d\left(R^{*}, C^{*}\right)_{\frac{1}{2}}} \\
& =\overline{d(\bar{C}, \bar{R})_{\frac{1}{2}}} \\
& =d(C, R)_{\frac{1}{2}} \\
& =d(R, c)_{\frac{1}{2}} \\
& =d O H
\end{aligned}
$$

Observation 6.6.9. We notice that the complete isomorphism of Proposition 6.6.8 is NOT the canonical identification between $\ell_{2}$ and its conjugate dual. Indeed, if $i: \overline{\ell_{2}^{*}} \rightarrow \ell_{2}$ is the canonical identification and we see $d O H$ as $\ell_{2} \oplus \ell_{2}$ as a Banach space then the identification of Proposition 6.6.8 is given by

$$
\left(\overline{x^{*}}, \overline{y^{*}}\right) \mapsto\left(i\left(\overline{x^{*}}\right),-i\left(\overline{y^{*}}\right)\right)
$$

We finish this section with a discussion on the algebra structure of the spaces $\mathrm{dOH}$ and $d o\left(\ell_{2}\right)$. Recall from Section 4.10 that an operator algebra $X$ is an operator space which is also a 
Banach algebra such that there is a complete isomorphism $T: X \rightarrow T(X) \subset B(H)$ which is an algebra homomorphism.

Recall from Theorem 4.10.4 that operator algebras are stable under interpolation. This is a consequence of Theorem 4.10.3. Indeed, if $\bar{X}=\left(X_{0}, X_{1}\right)$ is a compatible couple of operator spaces and $\sum(\bar{X})$ is endowed with a multiplication $m$ such that $\left(X_{0}, m\right)$ and $\left(X_{1}, m\right)$ are operator algebras, then $\mathscr{F}(\bar{X})$ is also an operator algebra, and therefore $X_{\theta}=\mathscr{F} / \operatorname{ker}\left(\delta_{\theta}\right)$ is an operator algebra.

The previous argument also proves:

Theorem 6.6.10. Let $\bar{X}=\left(X_{0}, X_{1}\right)$ be a compatible couple of operator spaces and $\sum(\bar{X})$ is endowed with a multiplication $m$ such that $\left(X_{0}, m\right)$ and $\left(X_{1}, m\right)$ are operator algebras. For every $\theta \in(0,1)$ the operator space $d X_{\theta}$ is an operator algebra with respect to the multiplication induced by the identification $d X_{\theta}=\mathscr{F} /\left(\operatorname{ker}\left(\delta_{\theta}\right) \cap \operatorname{ker}\left(\delta_{\theta}^{\prime}\right)\right)$.

The space $\ell_{2}$ is a Banach algebra with respect to pointwise multiplication. Some classical examples of operator algebras are the following:

Proposition 6.6.11 ((BLECHER; LE MERDY, 1995), Theorem 2.1(i)). $R, C, O H$ and $\max \left(\ell_{2}\right)$ are operator algebras.

So, by Theorem 6.6.10, we have

Theorem 6.6.12. The space $d O H$ is an operator algebra with respect to its natural product from the identification $d O H=\mathscr{F}(R, C) /\left(\operatorname{ker}\left(\delta_{\frac{1}{2}}\right) \cap \operatorname{ker}\left(\delta_{\frac{1}{2}}^{\prime}\right)\right)$.

However

Proposition 6.6.13 ((BLECHER; LE MERDY, 1995), Theorem 2.1(ii)). $\min \left(\ell_{2}\right)$ is not an operator algebra.

Accordingly, we have

Theorem 6.6.14. The operator space $d o\left(\ell_{2}\right)$ is not an operator algebra with respect to its natural product.

Proof. We must prove that the multiplication on $d o\left(\ell_{2}\right)$ is not completely bounded with respect to the Haagerup tensor product. The multiplication on $d o\left(\ell_{2}\right)$ is the one induced by the identification

$$
d o\left(\ell_{2}\right)=\mathscr{F}\left(\min \left(\ell_{2}\right), \max \left(\ell_{2}\right)\right) /\left(\operatorname{ker}\left(\delta_{\frac{1}{2}}\right) \cap \operatorname{ker}\left(\delta_{\frac{1}{2}}^{\prime}\right)\right)
$$

that is

$$
\left(x_{1}, y_{1}\right)\left(x_{2}, y_{2}\right)=\left(x_{1} x_{2}, x_{1} y_{2}+x_{2} y_{1}\right)
$$

for every $x_{i}, y_{i} \in \ell_{2}, i=1,2$.

Let $x^{n}$ be the matrices from Notation 6.5.3. Recall from Lemmata 6.5.4, 6.5.6, 6.5.7 and Corollary 6.5 .8 that

$$
\begin{aligned}
\left\|x^{n}\right\|_{M_{n}\left(\min \left(\ell_{2}\right)\right)} & =1 \\
\left\|x^{n}\right\|_{M_{n}\left(\max \left(\ell_{2}\right)\right)} & =n^{\frac{1}{2}} \\
\left\|x^{n}\right\|_{M_{n}(O H)} & =n^{\frac{1}{4}} \\
\left\|\left(x^{n}, \log \left(n^{-\frac{1}{2}}\right) x^{n}\right)\right\|_{d o\left(\ell_{2}\right)} & =n^{\frac{1}{4}}
\end{aligned}
$$


We notice that the same equalities are true for $\left(x^{n}\right)^{T}$ in place of $x^{n}$, the proofs are the same.

Notice also that $x^{n}\left(x^{n}\right)^{T}$ is the matrix with first entry $e_{1}+\ldots+e_{n}$ and zero elsewhere, so it has norm $n^{\frac{1}{2}}$.

If multiplication on $d o\left(\ell_{2}\right)$ was completely bounded, we would have for some constant $C>0$ and every $n \geq 1$

$$
\left\|\left(x^{n}, \log \left(n^{-\frac{1}{2}}\right) x^{n}\right)\left(\left(x^{n}\right)^{T}, \log \left(n^{-\frac{1}{2}}\right)\left(x^{n}\right)^{T}\right)\right\|_{M_{n}\left(d o\left(\ell_{2}\right)\right)} \leq C n^{\frac{1}{2}}
$$

However, if we take the constant function on $\mathbb{S}$ with value $x^{n}\left(x^{n}\right)^{T}$, there is a constant $\beta>0$ such that

$$
\begin{aligned}
\left\|\left(x^{n}, \log \left(n^{-\frac{1}{2}}\right) x^{n}\right)\left(\left(x^{n}\right)^{T}, \log \left(n^{-\frac{1}{2}}\right)\left(x^{n}\right)^{T}\right)\right\|_{M_{n}\left(d o\left(\ell_{2}\right)\right)} & = \\
\left\|\left(x^{n}\left(x^{n}\right)^{T}, \log \left(n^{-1}\right) x^{n}\left(x^{n}\right)^{T}\right)\right\|_{M_{n}\left(d o\left(\ell_{2}\right)\right)} & \geq \\
\left\|\left(0, \log \left(n^{-1}\right) x^{n}\left(x^{n}\right)^{T}\right)\right\|_{M_{n}\left(d o\left(\ell_{2}\right)\right)}-\left\|\left(x^{n}\left(x^{n}\right)^{T}, 0\right)\right\|_{M_{n}\left(d o\left(\ell_{2}\right)\right)} & = \\
n^{\frac{1}{2}}(\beta \log n-1) &
\end{aligned}
$$

so that we would have

$$
\beta \log n-1 \leq C
$$

for every $n$, a contradiction.

\subsection{An example with families}

Now we will consider two interpolation families of operator spaces. Let $A_{j}=\left[\frac{j}{4}, \frac{j+1}{4}\right)$, $j=0,1,2,3$, and consider the families $\mathscr{X}=\left\{X_{z}: z \in \mathbb{T} ; X\right\}$ and $\mathscr{Y}=\left\{Y_{z}: z \in \mathbb{T} ; Y\right\}$, where

$$
X_{e^{i t}}= \begin{cases}R & \text { if } t \in A_{0} \\ \max \left(\ell_{2}\right) & \text { if } t \in A_{1} \\ C & \text { if } t \in A_{2} \\ \min \left(\ell_{2}\right) & \text { if } t \in A_{3}\end{cases}
$$

$Y_{e^{i t}}=\bar{X}_{e^{i(t+\pi)}}$ and $X=Y=\ell_{2}$.

Then $\mathscr{X}$ and $\mathscr{Y}$ are interpolation families of operator spaces with containing space $\ell_{2}$ and containing function $k \equiv 1$.

We begin by identifying the space $X_{0}$.

Lemma 6.7.1. Let $f: \mathbb{T} \rightarrow \ell_{2}$ be an essentially bounded function such that its Fourier series contains only terms corresponding to non-negative indexes. Suppose that $f(z) \in\left[e_{1}, \ldots, e_{n}\right]$ for every $z \in \mathbb{T}$. Then $f$ extends to a function $F \in \mathscr{F}(\mathscr{Y})$ with the same bound of $f$.

Proof. We prove the result supposing that the image of $f$ is one-dimensional, the result for higher dimensions being a straightforward adaptation. Since $f$ is bounded, its Fourier coefficients are also bounded, and therefore its series converges absolutely for $|z|<1$, and the function $F$ so defined is in $H^{\infty}$.

We know that $F$ has a. e. nontangential limits on $\mathbb{T}$. Since $f \in L^{2}(\mathbb{T})$, it follows that its Fourier series converges to $f$ for a. e. $z \in \mathbb{T}$. A simple argument now shows that $\left.F\right|_{\mathbb{T}}=f$ a. e. 
Lemma 6.7.2. Let $\left(S_{j}, \mu_{j}\right)$ be finite measure spaces, and let $X_{j}$ be Hilbertian operator spaces, $j=1, \ldots, N$. Let $n \in \mathbb{N}$ and $T:\left(\oplus_{j=1}^{N} L_{1}\left(X_{j}, S_{j}, \mu_{j}\right)\right)_{1} \rightarrow M_{n}$ be a completely bounded operator. Then there is $S=\left(s_{j}\right)_{j=1}^{N} \in\left(\oplus_{j=1}^{N} L_{\infty}\left(M_{n}\left(X_{j}^{*}\right), S_{j}, \mu_{j}\right)\right)_{\infty}$ such that

$$
T\left(f_{j}\right)_{j=1}^{N}=\sum_{j=1}^{N} \int_{S_{j}} s_{j}(t)\left(f_{j}(t)\right) d \mu_{j}(t)
$$

for every $\left(f_{j}\right)$, and $\|S\| \leq\|T\|_{c b}$.

Proof. Let $P_{i, j}: M_{n} \rightarrow \mathbb{C}$ be projection of the $i, j$-entry. For $k \in\{1, \ldots, N\}, 1 \leq i, j \leq n$ and $l \in \mathbb{N}$ let

$$
\left(s_{k}\right)_{i, j}(z)(l)=P_{i, j} T\left(0, \ldots, e_{l}, \ldots, 0\right)
$$

where $e_{l}$ is in the $k$-th coordinate. Then $S=\left(s_{j}\right)_{j=1}^{N} \in\left(\oplus_{j=1}^{N} L_{\infty}\left(M_{n}\left(X_{j}^{*}\right), S_{j}, \mu_{j}\right)\right)_{\infty}$ satisfies (6.10) and for $f \in M_{m}\left(X_{k}\right)$ we have

$$
\left\|\left(s_{k}\right)_{m}(f)\right\|_{M_{n m}}=\left\|T_{m}(0, \ldots, f, \ldots, 0)\right\| \leq\|T\|_{c b}\|f\|
$$

so that $\left\|s_{k}\right\|_{M_{n}\left(X_{k}^{*}\right)} \leq\|T\|_{c b}$.

Lemma 6.7.3. We have completely isometrically $X_{0}^{*}=Y_{0}$.

Proof. Clearly as Banach spaces $X_{0}=\bar{Y}_{0}=\ell_{2}$. Let $n \in \mathbb{N}$ and $\bar{y} \in M_{n}\left(Y_{0}\right)$. Take $f \in \mathscr{G}(\mathscr{Y})$ such that $f(0)=\bar{y}$. By Proposition 2.5 of (COIFMAN et al., 1982), we may suppose that the coefficient functions of $f$ are in $H^{\infty}$.

Given $m \in \mathbb{N}$ and $x \in M_{m}\left(X_{0}\right)$, we also take $g \in \mathscr{G}\left(\mathscr{X}_{m}\right)$ with $g(0)=x$ and bounded coefficient functions, and let $h: \mathbb{D} \rightarrow M_{m n}$ be given by

$$
h(z)=(f(z))_{m}(g(z))
$$

Then $h$ is analytic and bounded, and by the duality relations between $R, C, \min \left(\ell_{2}\right)$ and $\max \left(\ell_{2}\right)$, we have that

$$
\|h(0)\| \leq\|f\|\|g\|
$$

Since $f$ and $g$ were arbitrary, we have

$$
\left\|\bar{y}_{m}(x)\right\|_{M_{m n}} \leq\|\bar{y}\|_{M_{n}\left(Y_{0}\right)}\|x\|_{M_{m}\left(X_{0}\right)}
$$

so that $\|y\|_{M_{n}\left(X_{0}^{*}\right)} \leq\|\bar{y}\|_{M_{n}\left(Y_{0}\right)}$. For the other inequality, let $y \in M_{n}\left(X_{0}^{*}\right)$ with finite support, $\lambda_{j}=d t$ restricted to $A_{j}$, and consider the space

$$
E=\left\{\left(f_{j}\right) \in\left(\oplus_{j=0}^{3} L_{1}\left(X_{j}, A_{j}, \lambda_{j}\right)\right)_{1}: \exists f \in \mathscr{F}(\mathscr{X}) \text { such that }\left.f\right|_{A_{j}}=f_{j}, j=0,1,2,3\right\}
$$

Then Proposition 2.3.68 and the universal property of the $L_{1}$-direct sum of operator spaces ((PISIER, 2003), page 52) imply that the operator $T: E \rightarrow M_{n}$ given by

$$
T\left(f_{j}\right)=\bar{y}(f(0))
$$

is completely bounded with $\|T\|_{c b}=\|y\|_{M_{n}\left(X_{0}^{*}\right)}$. By the Hahn-Banach theorem for operator spaces ((PISIER, 2003), Corollary 1.7) we may suppose that $T$ is defined on $\left(\oplus_{j=0}^{3} L_{1}\left(X_{j}, A_{j}, \lambda_{j}\right)\right)_{1}$. By Lemma 6.7.2, there is a function $S \in\left(\oplus_{j=0}^{3} L_{\infty}\left(M_{n}\left(X_{j}^{*}\right), A_{j}, \lambda_{j}\right)\right)_{\infty}$ defining $T$. Then $S$ may be seem as an essentially bounded function on $\mathbb{T}$, and its image has finite support.

Since $S$ represents $T$, we have that the Fourier series of each coefficient function of $S$ (when we see it as a function from $\mathbb{T}$ into $M_{n}\left(\ell_{2}\right)$ ) has only nonzero terms associated to 
nonnegative indexes (see the argument in the proof of Theorem 4.5.1 of (BERGH; LÖFSTRÖM, 1976)).

By Lemma 6.7.1, there is a function $g \in \mathscr{F}\left(\mathscr{Y}_{n}\right)$ with $S$ as boundary value a. e., and by (6.10), $g(0)=\bar{y}$, and therefore $\|\bar{y}\|_{M_{n}\left(Y_{0}\right)} \leq\|y\|_{M_{n}\left(X_{0}^{*}\right)}$.

However, since $\mathscr{Y}$ is obtained by a rotation of $\mathscr{X}$, we have that $X_{0}=\bar{Y}_{0}$ completely isometrically. Therefore, by the uniqueness of the operator Hilbert space, we have

Theorem 6.7.4. $X_{0}=O H$ completely isometrically.

The techniques from the previous sections adapt directly to this family so that

Theorem 6.7.5. We have a completely singular extension sequence induced by interpolation

$$
0 \longrightarrow \mathrm{OH} \longrightarrow \mathrm{dX} \longrightarrow \mathrm{OH} \longrightarrow 0
$$

\subsection{Saturation}

Following the classical setting, we say that a property $P$ of operator spaces which is stable under complete isomorphisms is a complete 3-space property ( $C 3 S P$ for short) if whenever we have an extension sequence

$$
0 \longrightarrow Y \longrightarrow X \longrightarrow Z \longrightarrow 0
$$

where $Y$ and $Z$ have $P$, then $X$ has $P$.

Definition 6.8.1. Let $Y$ be an infinite dimensional Banach space. An infinite dimensional Banach space $X$ is $Y$-saturated if every closed infinite dimensional subspace of $X$ has an isomorphic copy of $Y$.

Theorem 6.8.2 ((LINDENSTRAUSS; TZAFRIRI, 1996), Proposition I.2.a.2). For every $p \in$ $[1, \infty)$ the space $\ell_{p}$ is $\ell_{p}$-saturated.

The following result may be found in (CASTILLO; GONZÁLEZ, 1997) as Theorem 3.2.d.

Theorem 6.8.3. Being $\ell_{p}$-saturated is a $3 S P$.

Definition 6.8.4. Let $Y$ be an infinite dimensional operator space. An infinite dimensional operator space $X$ is $Y$-saturated if every closed infinite dimensional subspace of $X$ has a completely isomorphic copy of $Y$.

As a corollary to Lemma 6.5.2 we get

Theorem 6.8.5. For each $p \in[1, \infty)$, and $\theta \in(0,1)$, the space $o \ell_{p}(\theta)$ is $o \ell_{p}(\theta)$-saturated.

However, we shall prove that being $o \ell_{p}(\theta)$-saturated is not a C3SP.

Theorem 6.8.6. For each $p \in[1, \infty)$, and $\theta \in(0,1)$, being $o \ell_{p}(\theta)$-completely saturated is not a C3SP. In particular, being $\mathrm{OH}$-completely saturated is not a C3SP.

This is a direct consequence of the Theorem 6.8 .5 and the following: 
Proposition 6.8.7. Let $1 \leq p<\infty, \theta \in(0,1)$ and $X=\left\{(x, 0): x \in o\left(\ell_{p}\right)(\theta)\right\} \subset d o\left(\ell_{p}\right)(\theta)$. Then $X$ is a closed infinite dimensional subspace of $d o\left(\ell_{p}\right)(\theta)$ which does not contain a completely isomorphic copy of $o \ell_{p}(\theta)$.

Proof. Suppose that $W$ is a subspace of $X$ completely isomorphic to $o\left(\ell_{p}\right)(\theta)$. Then there is an infinite dimensional subspace $W^{\prime}$ of $o\left(\ell_{p}\right)(\theta)$ such that

$$
W=\left\{(x, 0): x \in W^{\prime}\right\}
$$

Notice that $W^{\prime}$ is isometric to $W$, so it is complete. Let $W^{\prime \prime}$ be a closed infinite dimensional subspace of $W^{\prime}$ complemented in $\ell_{p}$, and let $P: \ell_{p} \rightarrow W^{\prime \prime}$ be an onto bounded projection.

Let now $(P, 0): W \rightarrow\left\{(x, 0): x \in W^{\prime \prime}\right\}$ be given by $(P, 0)(x, 0)=(P x, 0)$. Then $(P, 0)$ is a bounded projection. By our assumption that $W$ is completely isomorphic to $o \ell_{p}(\theta)$ and Lemma 6.5.2, we have that $\left\{(x, 0): x \in W^{\prime \prime}\right\}$ is completely isomorphic to $o\left(\ell_{p}\right)(\theta)$. So, we may suppose that $W^{\prime}$ is itself complemented in $\ell_{p}$ and completely isomorphic to $o\left(\ell_{p}\right)(\theta)$.

Let $T: o\left(\ell_{p}\right)(\theta) \rightarrow W$ be a complete isomorphism. Then it must have the form $T=\left(T^{\prime}, 0\right)$ for some $T^{\prime}: \ell_{p} \rightarrow W^{\prime}$, and $T^{\prime}$ must be an isomorphism.

Since $W^{\prime}$ is complemented in $\ell_{p}$, we have by Lemma 6.5.2 that $T^{\prime}$ is a complete isomorphism between $o \ell_{p}(\theta)$ and $W^{\prime}$.

Since $T$ is completely bounded, we have for every $n$ and every $x \in M_{n}\left(o\left(\ell_{p}\right)(\theta)\right)$

$$
\left\|\left(T_{n}^{\prime} x, 0\right)\right\|=\left\|T_{n} x\right\| \leq\|T\|_{c b}\|x\|
$$

On the other hand, let $f \in M_{n}(\mathscr{F})$ be a function such that $f(\theta)=T_{n}^{\prime} x$ and $f^{\prime}(\theta)=0$. Let $g=\left(T_{n}^{\prime}\right)^{-1} P_{n} f$. Then $g \in M_{n}(\mathscr{F}), g(\theta)=x$ and $g^{\prime}(\theta)=0$, and $\|g\| \leq\left\|\left(T^{\prime}\right)^{-1}\right\|_{c b}\|P\|\|f\|$, from the homogeneity of min and max. Therefore

$$
\|(x, 0)\| \leq\left\|\left(T^{\prime}\right)^{-1}\right\|\|P\|\left\|\left(T_{n}^{\prime} x, 0\right)\right\|
$$

From (6.11) and (6.12) there is a constant $C>0$ such that

$$
\|(x, 0)\| \leq C\|x\|
$$

for every $n \in \mathbb{N}$ and every $x \in M_{n}\left(o \ell_{p}(\theta)\right)$. At this point, it should be clear that this is a contradiction to Corollary 6.5.8.

Observation 6.8.8. One may wonder why the proof that being $\ell_{p}$-saturated is a $3 \mathrm{SP}$ does not pass to the quantum case. The breaking point for the proof from (CASTILLO; GONZÁLEZ, 1997), for example, is the following: let $N$ and $M$ be closed subspaces of a Banach space $X$. If $N+M$ is closed, then we have the isomorphic identification:

$$
\frac{N+M}{N} \cong \frac{M}{M \cap N}
$$

If we let $X$ be as in Proposition 6.8.7 and $Y=\left\{(0, y) \in d O \ell_{p}(\theta): y \in O \ell_{p}(\theta)\right\}$, then $(X+Y) / Y=O \ell_{p}(\theta)$ completely isomorphically, but Proposition 6.8.7 tells us that $X=X /(X \cap$ $Y)$ is not completely isomorphic to $o \ell_{p}(\theta)$. That is, the second isomorphism theorem does not need to hold for operator spaces, even when it holds in the Banach setting. 


\subsection{Open problems}

If we have an extension sequence

$$
0 \longrightarrow Y \longrightarrow X \longrightarrow Z \longrightarrow 0
$$

then we can consider the corresponding sequence

$$
0 \longrightarrow \mathscr{K} \otimes_{\min } Y \longrightarrow \mathscr{K} \otimes_{\min } X \longrightarrow \mathscr{K} \otimes_{\min } Z \longrightarrow 0
$$

for which we have an associated quasilinear map $F: \mathscr{K} \otimes_{\min } Z \rightarrow \mathscr{K} \otimes_{\min } Y$, which may be seen as a collection of quasilinear maps $F^{n}: M_{n}(Z) \rightarrow M_{n}(Y)$ pasted together.

Problem 6.9.1. What is the corresponding notion of quasilinear map in operator space theory?

Problem 6.9.2. What is the corresponding notion of quasi-norm in operator space theory?

From Proposition 5.1.15 we may ask

Problem 6.9.3. For which extension sequences

$$
0 \longrightarrow Y \longrightarrow X \longrightarrow Z \longrightarrow 0
$$

are triviality and complete triviality equivalent?

Our results on the spaces $o\left(\ell_{p}\right)$ suggest

Problem 6.9.4. For which Banach spaces $X$ the extension sequence

$$
0 \longrightarrow o(X) \longrightarrow d o(X) \longrightarrow o(X) \longrightarrow 0
$$

is not completely trivial? For which $X$ is it completely singular/cosingular?

Finally, we have

Problem 6.9.5. Which properties of operator spaces are C3SP? Which are not? 

ALBIAC, F.; KALTON, N. J. Topics in Banach Space Theory. [S.1.]: Springer-Verlag New York, 2006. (Grad. Texts in Math.). Cited 3 times on pages 41, 42, and 46.

ANDERSON, J. M.; ROVNYAK, J. On Generalized Schwarz-Pick Estimates. Mathematika, v. 53, n. 1, p. 161-168, 2006. Cited on page 103.

AOKI, T. Locally bounded linear topological spaces. Proc. Imp. Acad., v. 18, n. 10, p. 588-594, 1942. Cited on page 11.

ARAZY, J.; LINDENSTRAUSS, J. Some linear topological properties of the spaces $C_{p}$ of operators on Hilbert space. Compos. Math., v. 30, n. 1, p. 81-111, 1975. Cited on page 61.

BERGH, J. On the Relation between the Two Complex Methods of Interpolation. Indiana Univ. Math. J., v. 28, p. 775-778, 1979. Cited on page 74.

BERGH, J.; LÖFSTRÖM, J. Interpolation Spaces: An Introduction. [S.1.]: Springer-Verlag Berlin Heidelberg, 1976. (Grundlehren Math. Wiss.). Cited 4 times on pages 15, 16, 20, and 131.

BLECHER, D. P. The standard dual of an operator space. Pacific J. Math., v. 153, n. 1, p. 15-30, 1992. Cited 2 times on pages 75 and 108.

. A completely bounded characterization of operator algebras. Math. Ann., v. 303, n. 1, p. 227-239, Sep 1995. Cited on page 79.

BLECHER, D. P.; LE MERDY, C. On quotients of function algebras and operator algebra structures on $\ell_{p}$. J. Operator Theory, v. 34, n. 2, p. 315-346, 1995. Cited 3 times on pages 80, 111 , and 128.

BLECHER, D. P.; RUAN, Z.-J.; SINCLAIR, A. M. A characterization of operator algebras. J. Funct. Anal., v. 89, n. 1, p. $188-201,1990$. Cited on page 80.

CABELLO SÁNCHEZ, F. There is no strictly singular centralizer on $L_{p}$. Proc. Amer. Math. Soc., v. 142 , n. 3, p. 949-955, 2014. Cited on page 59.

CABELlO SÁNCHEZ, F.; CASTILLO, J. M. F. Duality and Twisted Sums of Banach Spaces. J. Funct. Anal., v. 175, n. 1, p. 1 - 16, 2000. Cited on page 91.

CABELlO SÁNCHEZ, F.; CASTILLO, J. M. F.; GOLDSTEIN, S.; SUÁREZ DE LA FUENTE, J. Twisting non-commutative $L^{p}$ spaces. Adv. Math., v. 294, p. $454-488,2016$. Cited on page 113.

CALDERÓN, A. Intermediate spaces and interpolation, the complex method. Studia Math., v. 24, n. 2, p. 113-190, 1964. Cited 5 times on pages 9, 15, 17, 54, and 56.

CARRO, M. J.; CERDÁ, J.; SORIA, J. Commutators and interpolation methods. Ark. Mat., v. 33, n. 2, p. 199-216, 10 1995. Cited on page 17. 
CASTILLO, J. M. F.; CORRÊA, W. H. G.; FERENCZI, V.; GONZÁLEZ, M. Stability properties of the differential process generated by complex interpolation. ArXiv e-prints, dez. 2017. Cited on page 66.

CASTILLO, J. M. F.; CUELLAR, W.; FERENCZI, V.; MORENO, Y. Complex structures on twisted Hilbert spaces. Israel J. Math., v. 222, n. 2, p. 787-814, Oct 2017. Cited on page 3.

CASTILLO, J. M. F.; FERENCZI, V.; GONZÁLEZ, M. Singular twisted sums generated by complex interpolation. Trans. Amer. Math. Soc., n. 369, p. 4671-4708, 2017. Cited 9 times on pages $3,5,41,47,49,59,60,61$, and 66 .

CASTILLO, J. M. F.; FERENCZI, V.; MORENO, Y. On Uniformly Finitely Extensible Banach spaces. J. Math. Anal. Appl., v. 410, n. 2, p. 670-686, 2014. Cited on page 60.

CASTILlO, J. M. F.; GONZÁLEZ, M. Three-space Problems in Banach Space Theory. [S.1.]: Springer-Verlag Berlin Heidelberg, 1997. (Lecture Notes in Econom. and Math. Systems, 1667). Cited 9 times on pages 1, 9, 11, 12, 13, 14, 18, 131, and 132 .

CASTILLO, J. M. F.; MORENO, Y. Strictly singular quasi-linear maps. Nonlinear Analysistheory Methods \& Applications, v. 49, p. 897-903, 2002. Cited on page 60.

. On the bounded approximation property in Banach spaces. Israel J. Math., v. 198, n. 1, p. 243-259, 2013. Cited on page 66.

CHEN, D. Y.; ZHENG, B. T. Three-space problems for the bounded compact approximation property. Acta Math. Sin. (Engl. Ser.), v. 29, n. 4, p. 625-632, 2013. Cited on page 66.

COIFMAN, R. R.; CWIKEL, M.; ROCHBERG, R.; SAGHER, Y.; WEISS, G. A theory of complex interpolation for families of Banach spaces. Adv. Math., v. 43, n. 3, p. 203 - 229, 1982. Cited 14 times on pages 5, 9, 20, 22, 23, 24, 25, 27, 28, 29, 34, 35, 37, and 130.

CORRÊA, W. H. G. Twisting Operator Spaces. Accepted in Trans. Amer. Math. Soc., 2018. Cited 3 times on pages 5, 86, and 100 .

. Type, cotype and twisted sums induced by complex interpolation. J. Funct. Anal., v. 274, n. 3, p. $797-825,2018$. Cited on page 5 .

CWIKEL, M.; JAWERTH, B.; MILMAN, M.; ROCHBERG, R. Differential estimates and commutators in interpolation theory. In: Analysis at Urbana. [S.1.]: Cambridge Univ. Press, 1989. v. 2, p. 170-220. ISBN 9781107360204. Cambridge Books Online. Cited on page 55.

DUREN, P. L. Theory of $H^{p}$ Spaces. [S.1.]: Dover Publications, 2000. (Dover books on mathematics). Cited 5 times on pages 22, 23, 24, 26, and 27.

EFFROS, E. G.; RUAN, Z. J. Operator Spaces. [S.1.]: Oxford Univ. Press, 2000. v. 23. (London Math. Soc. Monogr., v. 23). Cited 6 times on pages 5, 69, 73, 75, 77, and 106.

ENFLO, P.; LINDENSTRAUSS, J.; PISIER, G. On the "three space problem". Math. Scand., v. 36, n. 2, p. 199-210, 1975. Cited 3 times on pages 2, 4, and 10.

FERENCZI, V. A uniformly convex hereditarily indecomposable Banach space. Israel J. Math., v. 102, n. 1, p. 199-225, Dec 1997. Cited 4 times on pages 5, 37, 38, and 59.

FORREST, B.; WOOD, P. Cohomology and the Operator Space Structure of the Fourier Algebra and its Second Dual. Indiana Univ. Math. J., v. 50, p. 1217-1240, 2001. Cited on page 4. 
GARLING, D. J. H.; MONTGOMERY-SMITH, S. J. Complemented Subspaces of Spaces Obtained by Interpolation. J. Lond. Math. Soc., s2-44, n. 3, p. 503-513, 1991. Cited on page 54.

GELFAND, I.; NEUMARK, M. On the imbedding of normed rings into the ring of operators in Hilbert space. Rec. Math. [Mat. Sbornik] N.S., v. 12(54), n. 2, p. 197-217, 1943. Cited on page 71.

GODEFROY, G.; SAPHAR, P. D. Three-Space Problems for the Approximation Properties. Proc. Amer. Math. Soc., v. 105, n. 1, p. 70-75, 1989. Cited on page 66.

HOFFMANN-JØRGENSEN, J. The Law of Large Numbers and the Central Limit Theorem in Banach Spaces. Proceedings of the Seminar on Random Series, Convex Sets and Geometry of Banach Spaces, v. 24, n. 4, p. 74-99, 1975. Cited on page 41.

HYTÖNEN, T.; NEERVEN, J. van; VERAAR, M.; WEIS, L. Analysis in Banach Spaces. Volume I: Martingales and Littlewood-Paley Theory. [S.1.]: Springer International Publishing, 2016. (Ergebnisse der Mathematik und ihrer Grenzgebiete. 3. Folge / A Series of Modern Surveys in Mathematics). Cited on page 25.

KALTON, N. Some remarks on interpolation of families of quasi-Banach spaces. Analysis at Urbana II, 1989. Cited on page 45.

KALTON, N.; PECK, N.; ROBERTS, J. An F-space Sampler. [S.1.]: Cambridge Univ. Press, 1984. (Lecture note series). ISBN 9780521275859. Cited on page 11.

KALTON, N. J. The three space problem for locally bounded F-spaces. Compositio Math, v. 37, n. 3, p. 243-276, 1978. Cited 2 times on pages 2 and 11.

Differentials of Complex Interpolation Processes for Köthe Function Spaces. Trans. Amer. Math. Soc., v. 333, n. 2, p. 479-529, 1992. Cited 2 times on pages 1 and 3.

KALTON, N. J.; MONTGOMERY-SMITH, S. Chapter 26 - Interpolation of Banach Spaces. In: . [S.1.]: Elsevier Science B.V., 2003, (Handbook of the Geometry of Banach Spaces, v. 2). p. 1131 -1175 . Cited 4 times on pages 5, 9, 19, and 33 .

KALTON, N. J.; PECK, N. T. Twisted sums of sequence spaces and the three space problem. Trans. Amer. Math. Soc., v. 255, p. 1-30, 1979. Cited 7 times on pages 2, 3, 10, 12, 13, 14, and 15.

KRANTZ, S. G. Handbook of Complex Variables. [S.1.]: Birkhäuser Basel, 1999. Cited on page 56.

LINDENSTRAUSS, J.; TZAFRIRI, L. Classical Banach spaces I and II. [S.1.]: Springer-Verlag, 1996. (Classics in mathematics). Cited 3 times on pages 62, 63, and 131.

LIONS, J. L. Une construction d'espaces d'interpolation. C. R. Acad. Sci. Paris, v. 251, p. 1853-1855, 1960. Cited 2 times on pages 9 and 15.

MASHREGHI, J. Representation Theorems in Hardy Spaces. [S.1.]: Cambridge Univ. Press, 2009. (London Math. Soc. Stud. Texts). Cited on page 21.

MAUREY, B.; PISIER, G. Séries de variables aléatoires vectorielles indépendantes et propriétés géométriques des espaces de Banach. Studia Math., v. 58, n. 1, p. 45-90, 1976. Cited on page 48. 
PAULSEN, V. I. Representations of function algebras, abstract operator spaces, and Banach space geometry. Journal of Functional Analysis, v. 109, n. 1, p. 113 - 129, 1992. Cited 2 times on pages 74 and 75 .

. Relative Yoneda Cohomology for Operator Spaces. J. Funct. Anal., v. 157, n. 2, p. 358 393, 1998. Cited 2 times on pages 4 and 91.

. Completely Bounded Maps and Operator Algebras. [S.1.]: Cambridge Univ. Press, 2002. (Cambridge Stud. Adv. Math.). ISBN 9780521816694. Cited on page 125.

PISIER, G. Holomorphic Semi-Groups and the Geometry of Banach Spaces. Ann. of Math., v. 115, n. 2, p. 375-392, 1982. Cited on page 42.

. The Operator Hilbert Space OH, Complex Interpolation and Tensor Norms. [S.1.]: Amer. Math. Soc., 1996. (Mem. Amer. Math. Soc., v. 122). ISBN 9780821804742. Cited 6 times on pages $73,74,75,99,113$, and 118 .

. Non-commutative vector valued $L_{p}$-spaces and completely $p$-summing maps. [S.1.]: Astérisque. Soc. Math. France, 1998. v. 247. Cited 4 times on pages 77, 78, 79, and 113.

Introduction to Operator Space Theory. [S.1.]: Cambridge Univ. Press, 2003. v. 294. (London Math. Soc. Lecture Note Ser., v. 294). Cited 6 times on pages 5, 69, 71, 82, 108, and 130.

. Complex Interpolation between Hilbert, Banach and Operator Spaces. [S.1.: s.n.], 2008. (Mem. Amer. Math. Soc., 208). Cited on page 95.

. Martingales in Banach Spaces. [S.1.]: Cambridge Univ. Press, 2016. (Cambridge Stud. Adv. Math.). Cited on page 39.

RIBE, M. Examples for the Nonlocally Convex Three Space Problem. Proc. Amer. Math. Soc., v. 73, n. 3, p. 351-355, 1979. Cited on page 2.

RIESZ, M. Sur les maxima des formes bilinéaires et sur les fonctionnelles linéaires. Acta Math., v. 49, n. 3-4, p. 465-497, 1926. Cited on page 15.

ROCHBERG, R. Higher order estimates in complex interpolation theory. Pacific J. Math., v. 174, n. 1, p. 247-267, 1996. Cited 2 times on pages 5 and 9.

ROCHBERG, R.; WEISS, G. Derivatives of Analytic Families of Banach Spaces. Ann. of Math., v. 118, n. 2, p. 315-347, 1983. Cited 3 times on pages 55, 56, and 57.

ROLEWICZ, S. On a certain class of linear metric spaces. Bull. Acad. Polon. Sci. Cl. III., v. 5, p. 471-473, XL, 1957. Cited on page 11.

RUAN, Z. J. Subspaces of C*-algebras. J. Funct. Anal., v. 76, n. 1, p. 217-230, 1988. Cited on page 71 .

Injectivity of operator spaces. Trans. Amer. Math. Soc., n. 315, p. 89-104, 1989. Cited 2 times on pages 107 and 108.

RUDIN, W. Functional Analysis. [S.1.]: Mcgraw Hill Higher Education, 2nd edition, 1991. Cited on page 11 . 
SÁNCHEZ, F. C.; CASTILLO, J. M.; SUÁREZ, J. On strictly singular nonlinear centralizers. Nonlinear Anal., v. 75, n. 7, p. 3313-3321, 2012. Cited on page 60.

SMIRNOV, V. A.; KHUEN, C. On the function Ext in the category of linear topological spaces. Math. USSR Izvestiya, v. 36, p. 199-210, 1991. Cited on page 2.

SMIRNOV, V. A.; SHEIKHMAN, V. A. Continuation of homogeneous functionals with a given convexity. Mat. Zametki, v. 50, n. 5, p. 1157-1161, 1991. Cited on page 2.

STAFNEY, J. D. The Spectrum of an Operator on an Interpolation Space. Trans. Amer. Math. Soc., v. 144, p. 333-349, 1969. Cited 2 times on pages 32 and 56.

SUÁREZ DE LA FUENTE, J. The Kalton centralizer on $L_{p}[0,1]$ is not strictly singular. Proc. Amer. Math. Soc., v. 141, n. 10, p. 3447-3451, 2013. Cited on page 59.

. A remark about twisting Schatten classes. Rocky Mountain J. Math., v. 44, n. 6, p. $2093-$ 2102, 12 2014. Cited on page 61.

SZANKOWSKI, A. Subspaces without the approximation property. Israel J. Math, v. 30, n. 1, p. 123-129, 1978. Cited on page 61.

Three-space problems for the approximation property. J. Eur. Math. Soc. (JEMS), v. 011, n. 2, p. 273-282, 2009. Cited on page 66.

SZEGÖ, G. Über die Randwerte einer analytischen Funktion. Math. Ann., v. 84, p. 232-244, 1921. Cited on page 24.

THORIN, G. O. An extension of a convexity theorem due to M. Riesz. Kungl. Fysiogr. Sällsk. $i$ Lund Förh., v. 8, p. 166-170, 1938. Cited on page 15.

WOOD, P. J. Homological Algebra in Operator Spaces with Applications to Harmonic Analysis. 1999 (PhD thesis). Cited 4 times on pages 4, 81, 82, and 84. 